Florida International University

FIU Digital Commons

$10-18-2018$

\title{
Design Optimization of Inductive Power Transfer Systems for Contactless Electric Vehicle Charging Applications
}

Masood Moghaddami

Florida International University, mmogh003@fiu.edu

Follow this and additional works at: https://digitalcommons.fiu.edu/etd

Part of the Controls and Control Theory Commons, and the Electrical and Electronics Commons

\section{Recommended Citation}

Moghaddami, Masood, "Design Optimization of Inductive Power Transfer Systems for Contactless Electric Vehicle Charging Applications" (2018). FIU Electronic Theses and Dissertations. 3853.

https://digitalcommons.fiu.edu/etd/3853

This work is brought to you for free and open access by the University Graduate School at FIU Digital Commons. It has been accepted for inclusion in FIU Electronic Theses and Dissertations by an authorized administrator of FIU Digital Commons. For more information, please contact dcc@fiu.edu. 


\section{FLORIDA INTERNATIONAL UNIVERSITY \\ Miami, Florida}

DESIGN OPTIMIZATION OF INDUCTIVE POWER TRANSFER SYSTEMS FOR CONTACTLESS ELECTRIC VEHICLE CHARGING APPLICATIONS

A dissertation submitted in partial fulfillment of the

$$
\begin{gathered}
\text { requirements for the degree of } \\
\text { DOCTOR OF PHILOSOPHY } \\
\text { in } \\
\text { ELECTRICAL ENGINEERING } \\
\text { by }
\end{gathered}
$$

Masood Moghaddami 
To: Dean John L. Volakis

College of Engineering and Computing

This dissertation, written by Masood Moghaddami, and entitled Design Optimization of Inductive Power Transfer Systems for Contactless Electric Vehicle Charging Applications, having been approved in respect to style and intellectual content, is referred to you for judgment.

We have read this dissertation and recommend that it be approved.

Osama Mohammed

Nezih Pala

Sakhrat Khizroev

Francisco de León

Arif I. Sarwat, Major Professor

Date of Defense: October 18, 2018

The dissertation of Masood Moghaddami is approved.

Dean John L. Volakis

College of Engineering and Computing

Andrés G. Gil

Vice President for Research and Economic Development and Dean of the University Graduate School

Florida International University, 2018 
(C) Copyright 2018 by Masood Moghaddami

All rights reserved. 


\section{DEDICATION}

Dedicated to my parents Batoul and Youness, my wife Marzieh and my son Ali. 


\section{ACKNOWLEDGMENTS}

I would like to express my sincere gratitude to my advisor Dr. Arif Sarwat for the continuous support of my Ph.D study and related research, for his patience, and motivation. Besides my advisor, I would like to thank the rest of my thesis committee: Dr. Osama Mohammed, Dr. Nezih Pala, Dr. Sakhrat Khizroev and Dr. Francisco de Leon, for their insightful comments and encouragement to widen my research from various perspectives.

This dissertation was supported by the National Science Foundation under grant number 1541108 and FIU Graduate School Dissertation Year Fellowship (DYF). 


\title{
ABSTRACT OF THE DISSERTATION \\ DESIGN OPTIMIZATION OF INDUCTIVE POWER TRANSFER SYSTEMS FOR CONTACTLESS ELECTRIC VEHICLE CHARGING APPLICATIONS
}

by

\author{
Masood Moghaddami \\ Florida International University, 2018 \\ Miami, Florida \\ Professor Arif I. Sarwat, Major Professor
}

Contactless Electric Vehicle (EV) charging based on magnetic resonant induction is an emerging technology that can revolutionize the future of the EV industry and transportation systems by enabling an automated and convenient charging process. However, in order to make this technology an acceptable alternative for conventional plug-in charging systems it needs to be optimized for different design measures. Specifically, the efficiency of an inductive EV charging system is of a great importance and should be comparable to the efficiency of conventional plug-in EV chargers.

The aim of this study is to develop solutions that contribute to the design enhancement of inductive EV charging systems. Specifically, generalized physics-based design optimization methods that address the trade-off problem between several key objectives including efficiency, power density, misalignment tolerance, and cost efficiency considering critical constraints are developed. Using the developed design methodology, a $3.7 \mathrm{~kW}$ inductive charging system with square magnetic structures is investigated as a case study and a prototype is built to validate the optimization results. The developed prototype achieves $93.65 \%$ efficiency (DC-to-DC) and a power density of $1.65 \mathrm{~kW} / \mathrm{dm}^{3}$.

Also, self-tuning power transfer control methods with resonance frequency tracking capability and bidirectional power transfer control are presented. The proposed control methods enhance the efficiency of power converters and reduce the Electromagnetic Interference (EMI) by enabling soft-switching operations. Several simplified digital controllers 
are developed and experimentally implemented. The controllers are implemented without the use of DSP/FPGA solutions. Experimental tests show that of the developed simplified controllers can effectively regulate the power transfer around the desired value. Moreover, the experiments show that compared to conventional converters, the developed converters can achieve $4 \%$ higher efficiency at low power levels.

Moreover, enhanced matrix converter topologies that can achieve bidirectional power transfer and high efficiency with a reduced number of switching elements are introduced. The self-tuning controllers are utilized to design and develop control schemes for bidirectional power transfer regulation. The simulation analyses and experimental results show that the developed matrix converters can effectively establish bidirectional power transfer at the desired power levels with soft-switching operations and resonance frequency tracking capability. Specifically, a direct three-phase AC-AC matrix converter with a reduced number of switches (only seven) is developed and built. It is shown that the developed converters can achieve efficiencies as high as $98.54 \%$ at high power levels and outperform conventional two-stage converters. 


\section{TABLE OF CONTENTS}

1. INTRODUCTION . . . . . . . . . . . . . . . . . 1

1.1 Existing Challenges in Contactless Electric Vehicle Charging Systems . . 3

1.1.1 Power Transfer Efficiency . . . . . . . . . . . . . . . . . . . . 4

1.1.2 Power Density and Cost Efficiency . . . . . . . . . . . . . 5

1.1.3 Misalignment Tolerance . . . . . . . . . . . . . . . . . . . 7

1.1.4 Electromagnetic Field Emissions . . . . . . . . . . . . . . 7

1.1.5 Foreign Object Detection . . . . . . . . . . . . . . 7

1.2 Literature Review . . . . . . . . . . . . . . . . . . . . . . . . . 8

1.2.1 Magnetic Structures of Inductive Electric Vehicle Charging Systems . . 9

1.2.2 Power Converter Topologies in Inductive Power Transfer Systems . . . 10

1.2.3 Control Methods of Inductive Power Transfer Systems . . . . . . . . . 14

1.3 Research Objectives and Original Contributions . . . . . . . . . . . . 17

2. GENERALIZED PHYSICS-BASED MULTI-OBJECTIVE DESIGN OPTIMIZATION OF MAGNETIC STRUCTURES FOR INDUCTIVE CHARG-

ING SYSTEMS . . . . . . . . . . . . . . . . . . 23

2.1 Multi-Objective Design Optimization of Square Magnetic Structures for

Static Inductive Charging Systems _. . . . . . . . . . . . 24

2.1.1 Derivation of Equivalent Circuit Model of an Inductive Charging System 25

2.1.1.1 Equivalent Model of a Full-Wave Battery Charger . . . . . . . . . . . 26

2.1.1.2 Equivalent Circuit of Series Compensated Inductive Battery Charger 27

2.1.1.3 Inductive Charging System Efficiency . . . . . . . . . . . . . . . 28

2.1.1.4 Optimal Input Voltage . . . . . . . . . . . . . . . . . . . . . . 28

2.1.1.5 Output Power Constraint . . . . . . . . . . . . . . . . . . 30

2.1.2 Parametric Physics Model Using 3D Finite Element Model . . . . . . . 31

2.1.2.1 Equivalent Resistances of Coil . . . . . . . . . . . . . . . . . 32

2.1.2.2 Equivalent Resistance of Core Losses . . . . . . . . . . . . . . . . . . . 33

2.1.2.3 Equivalent Resistance of Stray Losses . . . . . . . . . . . . . . . . . 34

2.1.2.4 Equivalent Resistance of Compensation Capacitors . . . . . . . . . . 34

2.1.2.5 Thermal Performance Analysis . . . . . . . . . . . . . . . . 35

2.1.3 Multi-Objective Optimization of Magnetic Structure Design . . . . . . 35

2.1.3.1 Multi-objective Optimization Formulation . . . . . . . . . . . . 35

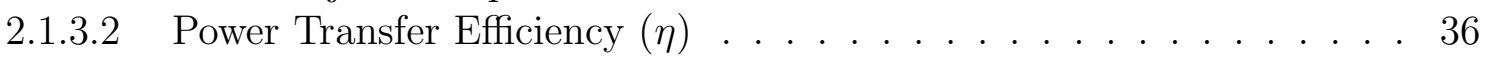

2.1.3.3 Horizontal Misalignment Tolerance $(\Delta) \ldots \ldots$. . . . . . . 36

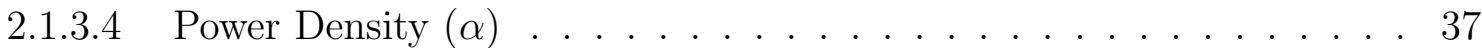

2.1.3.5 Specific Cost $(\beta) \ldots \ldots \ldots \ldots$

2.1.3.6 Genetic Algorithms for Multi-objective Optimization . . . . . . . . . 38

2.1.4 Case Study Analysis . . . . . . . . . . . . . . . . . . . . . . 40

2.1.4.1 Design for Efficiency . . . . . . . . . . . . . . . . . . 42 
2.1.4.2 Design for Efficiency and Power Density . . . . . . . . . . . . . . . . 42

2.1.4.3 Design for Power Density . . . . . . . . . . . . . . . . . . 43

2.1.4.4 Design for Efficiency and Displacement Tolerance . . . . . . . . . . . 43

2.1.4.5 Design for Cost Efficiency . . . . . . . . . . . . . . . . 43

2.1.5 Experimental Analysis . . . . . . . . . . . . . . . . 44

3. DESIGN AND DEVELOPMENT OF SELF-TUNING CONTROLLERS FOR

INDUCTIVE POWER TRANSFER SYSTEMS . . . . . . . . . . . . 46

3.1 Sliding Mode Controller for H-Bridge Converters in Inductive Power

Transfer Systems . . . . . . . . . . . . . . . . . . . 46

3.1.1 Self-Tuning Controller Design . . . . . . . . . . . . . . . . . 47

3.1.1.1 Sliding Mode Controller Design . . . . . . . . . . . . . . . . . . 48

3.1.1.2 Simplified Design for the Sliding Mode Controller . . . . . . . . . . . 51

3.1.2 Simulation Analysis . . . . . . . . . . . . . . . . . . 52

3.1.3 Experimental Analysis . . . . . . . . . . . . . . . 54

3.2 Self-Tuning Variable Frequency Controller for Inductive Electric Vehicle

Charging with Multiple Power Levels . . . . . . . . . . . . . . . . . 54

3.2.1 The Proposed Controller . . . . . . . . . . . . . . . . 56

3.2 Theoretical Analysis . . . . . . . . . . . . . . . . . . 60

3.2.2.1 Resonant Current and Resonant Voltage Calculation . . . . . . . . . 60

3.2.2.2 Output Power Calculation . . . . . . . . . . . . . . . . . 62

3.2.3 Simulation Results . . . . . . . . . . . . . . . . . . . . 63

3.2.4 Experimental Analysis . . . . . . . . . . . . . . . 65

3.3 Development of a Power-Frequency Controller with Resonance Frequency

Tracking Capability for Inductive Power Transfer Systems . . . . . . 67

3.3.1 Proposed Control Method . . . . . . . . . . . . . . . . 69

3.3.1.1 Power-Frequency Controller . . . . . . . . . . . . . . 70

3.3.1.2 Self-Tuning Capability . . . . . . . . . . . . . . . . 72

3.3.2 Theoretical Analysis . . . . . . . . . . . . . . . . . . . . 74

3.3.2.1 Power Transfer Level . . . . . . . . . . . . . . . . . . . . 74

3.3.2.2 Voltage Gain of the Converter . . . . . . . . . . . . . . . . 77

3.3.2.3 System Response Time . . . . . . . . . . . . . . . . . . . . . 78

3.3.2.4 Steady-State Error . . . . . . . . . . . . . . . . 78

3.3.3 Simulation Results . . . . . . . . . . . . . . . . . . 79

3.3.4 Experimental Results . . . . . . . . . . . . . . . . . . 84

3.3.4.1 Efficiency Analysis . . . . . . . . . . . . . . . . . . 87

3.3.4.2 Resonance Frequency Tracking Capability Analysis . . . . . . . . . . 88

3.4 Summary . . . . . . . . . . . . . . . . . . . . . . . . 90

4. SINGLE-PHASE SOFT-SWITCHED AC-AC MATRIX CONVERTERS WITH POWER CONTROLLER FOR BIDIRECTIONAL INDUCTIVE POWER

TRANSFER SYSTEMS . . . . . . . . . . . . . . . . . 91 
4.1 Self-Tuned Single-Phase AC-AC Converter for Bidirectional Inductive Power Transfer Systems . . . . . . . . . . . . . . . . . . 92

4.1.1 Sliding Mode Controller Design for Single-Phase AC-AC Converter . . 93

4.1.1.1 Sliding Mode Control Law Derivation . . . . . . . . . . . . . . . 93

4.1 .2 Simulation Results . . . . . . . . . . . . . . . . . . . . . . . 100

4.2 Single-Phase Soft-Switched AC-AC Matrix Converter with Power Controller for Bidirectional Inductive Power Transfer Systems . . . . . . . 102

4.2.1 Power Transfer Controller for Direct Single-Phase AC-AC Converter 103

4.2.1.1 Quantum Energy Injection/Regeneration Principle for Power Transfer Control . . . . . . . . . . . . 106

4.2.1.2 Switching Logic Design _ . . . . . . . . . . . . . . . . . . . 107

4.2.1.3 Power Transfer Controller . . . . . . . . . . . . . . . . . . . . . . . 110

4.2.1.4 Forward Power Transfer . . . . . . . . . . . . . . . . . . . . . . . 111

4.2.1.5 Reverse Power Transfer . . . . . . . . . . . . . . . . . . . . . . . . . 112

4.2 .2 Theoretical Analysis . . . . . . . . . . . . . . . . . . . . . . 113

4.2.2.1 Calculation of Power Transfer . . . . . . . . . . . . . . . . . . 113

4.2.2.2 Calculation of Voltage Transfer Ratio (VTR) . . . . . . . . . . . . 115

4.2.2.3 Efficiency Analysis . . . . . . . . . . . . . . . . . . . . 116

4.2 .3 Simulation Results . . . . . . . . . . . . . . . . . . . . . . 117

4.2 .4 Experimental Results . . . . . . . . . . . . . . . . . . . . . . . 122

4.3 Summary . . . . . . . . . . . . . . . . . . . . . 125

5. THREE-PHASE AC-AC MATRIX CONVERTER FOR INDUCTIVE POWER

TRANSFER SYSTEM . . . . . . . . . . . . . . . . . 127

5.1 Single-Stage Three-Phase AC-AC Matrix Converter for Inductive Power

Transfer Systems . . . . . . . . . . . . . . . . . . . 128

5.1.1 Proposed Three-Phase AC-AC Converter . . . . . . . . . . . . . . . 128

5.1.1.1 Current Regulation Control Mode . . . . . . . . . . . . . . . . . . 131

5.1.1.2 Voltage Regulation Control Mode . . . . . . . . . . . . . . . . . . . 132

5.1.1.3 Power Regulation Control Mode . . . . . . . . . . . . . . . . . . . 134

5.1 .2 Theoretical Analysis . . . . . . . . . . . . . . . . . . . . . 136

5.1.2.1 Converter Loss Analysis . . . . . . . . . . . . . . . . . . . . . . . . 139

5.1 .3 Simulation Results . . . . . . . . . . . . . . . . . . . . . . . . . . . 142

5.1 .4 Experimental Results . . . . . . . . . . . . . . . . . . . . . . . . 144

5.1.4.1 Current Regulation Control . . . . . . . . . . . . . . . . . . . . . 145

5.2 Three-Phase AC-AC Matrix Converter with Simplified Bidirectional Power

Control for Inductive Power Transfer Systems . . . . . . . . . . . . 148

5.2.1 Bidirectional Power Transfer Control for Three-Phase AC-AC Converter 149

5.2.2 Simulation Analysis and Experimental Results . . . . . . . . . . . . . 154

5.3 Summary . . . . . . . . . . . . . . . . . . . 156 
6. APPLICATIONS OF SELF-TUNING CONTROLLERS IN INDUCTIVE ELECTRIC VEHICLE CHARGING SYSTEMS . . . . . . . . . . . . . 159

6.1 Sensorless Conductive Foreign Object Detection for Inductive Electric

Vehicle Charging Systems Based on Resonance Frequency Deviation . 159

6.1.1 Effects of Conductive Foreign Objects on IPT Systems . . . . . . . . . 160

6.1.2 Conductive Foreign Object Detection Method . . . . . . . . . . . . . 161

6.1.2.1 Initial Foreign Object Detection at Low Power . . . . . . . . . . . 166

6.1.2.2 System Losses in Initial Foreign Object Detection Mode . . . . . . . 166

6.1.2.3 Online Foreign Object Detection Along With Charging Process . . 167

6.1.2.4 Foreign Object Detection Speed . . . . . . . . . . . . . . . . 167

6.1 .3 Experimental Analysis . . . . . . . . . . . . . . . . . . . . . . . 169

6.2 Sensorless Electric Vehicle Detection in Inductive Charging Stations Using Self-Tuning Controllers . . . . . . . . . . . . . . . . . 171

6.2.1 Proposed Vehicle Detection Method . . . . . . . . . . . . . . . . . 172

6.2 .2 Simulation Analysis . . . . . . . . . . . . . . . . . . . . 174

7. CONCLUSIONS AND FUTURE WORK . . . . . . . . . . . . . 177

7.1 Future Works . . . . . . . . . . . . . . . . . . . . . . . . 181

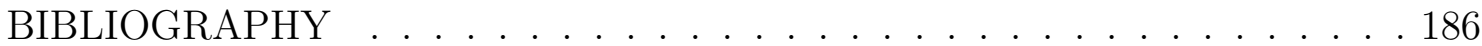

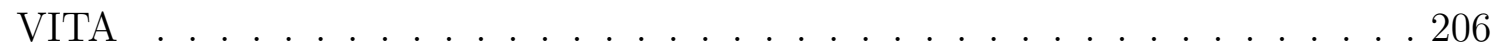




\section{LIST OF TABLES}

TABLE

PAGE

2.1 The cost coefficients for different materials. . . . . . . . . . . . . . 40

2.2 Design specifications of the target case study system. . . . . . . . . . . . 40

2.3 Optimization results for different design scenarios. . . . . . . . . . . . . 41

2.4 Specifications of the experimental setup. . . . . . . . . . . . . . 44

3.1 Four operation modes and corresponding switching states in an H-bridge converter. . . . . . . . . . . . . . . . 50

3.2 Four operation modes of a full-bridge inverter and corresponding switching states. . . . . . . . . . . . . . . . 5 56

3.3 Different charging levels which can be achieved using the proposed controller. . . . . . . . . . . . . . . . . . . 60

3.4 The simulation results at charging levels $1-1,1-2,2-2$ and $4-8 \ldots \ldots$. . . 63

3.5 Experimental test results on the case study IPT system using the proposed controller at different charging levels. . . . . . . . . . . . 67

3.6 Distinct power transfer levels and corresponding energy injection frequencies for positive and negative half-cycles. . . . . . . . . . . . . 69

3.7 Specifications of the case study IPT system. . . . . . . . . . . . . 80

3.8 Distinct power transfer levels and corresponding energy injection frequencies for positive and negative half-cycles. . . . . . . . . . 81

3.9 Specifications of the experimental IPT system. . . . . . . . . . . . 84

3.10 List of components used in experimental implementation of the proposed power controller. . . . . . . . . . . . . . . . . 84

4.1 Operation modes of the single-phase AC-AC converter. . . . . . . . . . . 99

4.2 Specifications of the case study system. . . . . . . . . . . . . . . 101

4.3 Operation modes and corresponding switching states of the single-phase AC-AC matrix converter . . . . . . . . . . . . . . . 107

4.4 Power transfer levels obtained using the power controller . . . . . . . . . 111

4.5 Specifications of the case study IPT system . . . . . . . . . . . 117

4.6 Simulation results of the case study IPT system in forward power transfer $(\mathrm{G} 2 \mathrm{~V})$ at different power levels . . . . . . . . . . . . . . . . 119 
4.7 Simulation results of the case study IPT system in reverse power transfer (V2G) at different power levels . . . . . . . . . . . . . 120

4.8 Specifications of the case study IPT system setup . . . . . . . . . . . 122

5.1 Switching States in different Modes of Operation in Current Regulation Control Mode. . . . . . . . . . . . . . . . . . . . 134

5.2 Switching States in different Modes of Operation in Voltage Regulation Control Mode. . . . . . . . . . . . . . . . . . . . . 134

5.3 Switching States in different Modes of Operation in Power Regulation Control Mode. . . . . . . . . . . . . . . . . . . . . 135

5.4 Typical values for parameters used for theoretical converter loss calculation. . . . . . . . . . . . . . . . . . . . . . 140

5.5 Switching states in forward power transfer mode . . . . . . . . . . . 154

5.6 Switching states in reverse power transfer mode . . . . . . . . . . . 155

6.1 Specifications of the case study system. . . . . . . . . . . . . . . . 169

6.2 Specifications of the case study inductive charging system. . . . . . . . 175 


\section{LIST OF FIGURES}

FIGURE

1.1 A typical contactless electric vehicle charging system based on inductive power transfer. . . . . . . . . . . . . . . . . 1

1.2 Typical structure of a dynamic (roadway) contactless EV charging system EV charging system. . . . . . . . . . . . . . 3

1.3 The structure of a bidirectional IPT system. . . . . . . . . . . . . . 12

2.1 The circuit diagram of series compensated inductive charging systems (a) complete model including primary and secondary power converters, (b) simplified fundamental harmonic model, (c) primary side model. . . . . . . . . . . . . . . . . . . . . . 25

2.2 Power transfer efficiency $(\eta)$ of an inductive charging system as a function of resistance $r$ and mutual inductance $M . \ldots \ldots . . \ldots 29$

2.3 Representation of circuit-based (power transfer) and physics-based constraints in $\omega M-r$ coordinates. . . . . . . . . . . . 30

2.4 Structure of a square magnetic coupler for inductive EV charging systems. 31

2.5 Boundary conditions of the hybrid physics model solved using FEA. . . 31

2.6 Sample finite element analysis results of the hybrid physics model: (a) Magnetic flux density distribution, (b) Temperature distribution. . . 32

2.7 The flowchart of the proposed physics-based multi-objective design optimization algorithm. . . . . . . . . . . . . 38

2.8 Optimized magnetic structures for different design cases of Table 2.3. . . 40

2.9 Contour plots of fitness function for different design cases of Table 2.3. . 41

2.10 The developed experimental setup with optimized square magnetic structures designed for high power density. . . . . . . . . . . . . . 44

2.11 Power transfer efficiency of the case study system as function of receiver power obtained using both calculations and experimental measurements with and without horizontal offset. . . . . . . . . . . . 44

3.1 An H-bridge converter connected to an equivalent RLC circuit representing an IPT system. . . . . . . . . . . . . . . . 48

3.2 Resonant current and output voltage of the H-bridge converter which is controlled using the proposed SMC based on energy injection and free oscillation technique. . . . . . . . . . . . . . 4 48 
3.3 Different control modes in an H-bridge converter: energy injection modes (1 and 2), free-oscillation modes (3 and 4). . . . . . . . . . 50

3.4 The proposed simplified controller for H-bridge resonant converters $\left(i_{r}\right.$ is the measured resonant current signal, $i_{\text {ref }}$ is the reference current). 51

3.5 Simulation results on the case study IPT system using an H-bridge converter topology: (a) $i_{r e f}=40 \mathrm{~A}, P_{\text {out }}=6.6 \mathrm{~kW}$, (b) $i_{\text {ref }}=60 \mathrm{~A}, P_{\text {out }}=14 \mathrm{~kW} .52$

3.6 The case study IPT system with circular pads as transmitter and receiver, an AC/DC/AC converter controlled by the proposed control

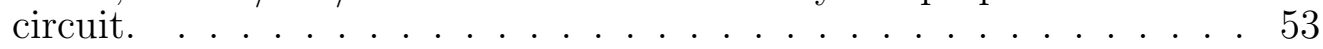

3.7 Resonant current and energy injection switching signals of the H-bridge converter in the case study IPT system: (a) $V_{L L}=10 \mathrm{~V}, i_{\text {ref }}=3.6 \mathrm{~A}$, $P_{\text {out }}=35 \mathrm{~W}$, (b) $V_{L L}=20 \mathrm{~V}, i_{\text {ref }}=10 \mathrm{~A}, P_{\text {out }}=155 \mathrm{~W} \ldots \ldots \ldots 3$

3.8 The proposed simplified control circuit designed for a two-stage fullbridge AC/DC/HFAC converter. . . . . . . . . . . . . . 55

3.9 The energy injection switching signals with frequency of $f_{r}$ (resonance frequency), $f_{r} / 2, f_{r} / 4$ and $f_{r} / 8$ for positive $\left(P_{1}, P_{2}, P_{3}\right.$ and $\left.P_{4}\right)$ and negative $\left(N_{1}, N_{2}, N_{3}\right.$ and $\left.N_{4}\right)$ half-cycles which are generated generated by the proposed controller. . . . . . . . . . . 57

3.10 Typical waveforms of the resonant current and the converter output voltage applying the proposed control method for charging Level 2-4. 57

3.11 Inductive electric vehicle battery charging model with the proposed controller simulated in MATLAB/Simulink. . . . . . . . . . . . 63

3.12 The resonant current, inverter output voltage, switching signals and battery charging current in different charging levels: (a) Level 1-1 charging with $41 \mathrm{~kW}$ output power, (b) Level 1-2 charging with 22.1 $\mathrm{kW}$ output power, (c) Level 2-2 charging with $12.3 \mathrm{~kW}$ output power, (d) Level 4-8 charging with $3.6 \mathrm{~kW}$ output power. . . . . . . . . . . . 64

3.13 The case study IPT setup consisting of two circular transmitter and receiver power pads, an $\mathrm{AC} / \mathrm{DC} / \mathrm{AC}$ converter as the primary converter and the proposed controller. . . . . . . . . . . . .

3.14 Experimental test results on the IPT system setup: the resonant current and energy-injection switching signals at different charging levels. . . 66

3.15 The proposed power-frequency controller designed based on a digital control circuit. . . . . . . . . . . . . . . . 70

3.16 Resonant current and corresponding positive and negative half-cycle energy injection signals for $n, m \in\{1,2,4,8\} \ldots \ldots \ldots 72$ 
3.17 The resonant current and corresponding switching signals controlled using the proposed controller for power level 2-4 $(n=2, m=4)$. . . . . 73

3.18 The output power and steady-state error as a function of reference power. . . . . . . . . . . . . . . . . . 79

3.19 The case study IPT system simulation model. . . . . . . . . . . . . 80

3.20 Power and efficiency of the converter at different power transfer levels calculated based on simulation results. . . . . . . . . . . . . . . 81

3.21 Simulation results on the case study IPT system showing resonant current, DC-link current, energy-injection switching signals $\left(S_{3}, S_{4}\right)$, reference power and output power. . . . . . . . . . . . . . 82

3.22 The experimental set-up of the proposed power-frequency controller. . . 83

3.23 Experimental setup of the proposed controller and its components. . . . 83

3.24 Experimental results on the case study IPT system at different power levels: (a) output power obtained based on experimental measurements and theoretical calculations, (b) converter efficiency obtained based on experimental measurements and simulation results. . . . . . 85

3.25 Experimental results on the IPT setup showing the resonant current, battery charging current and negative half-cycle switching signal $\left(S_{3}\right)$ : (a) transition from Level 1-8 to Level 1-1, (b) transition from Level 1-1 to Level 1-8. . . . . . . . . . . . . . . . . . . . . . . .

3.26 Experimental results on the IPT setup showing the resonant current, primary DC-link current and energy injection switching signals $\left(S_{3}\right.$ and $S_{4}$ ): (a) Level 1-4 power transfer, (b) Level 2-2 power transfer. . 86

3.27 The output voltage of the $\mathrm{DC} / \mathrm{AC}$ converter and resonant current at power Level $2-4 . \ldots \ldots \ldots$. . . . . . . . . . . . . 88

3.28 Experimental frequency measurement results: (a) Dynamic resonance frequency variations during horizontal movement of the secondary pad at $50 \mathrm{~mm} / \mathrm{s}$ speed and $200 \mathrm{~mm}$ air gap, (b) Resonance frequency measurements with variations in distance between the transmitter and receiver power pads (g: air gap). . . . . . . . . . . . . . .

4.1 Single-phase ac-ac matrix converter with the simplified self-tuning controller. . . . . . . . . . . . . . . . . . . . . . 92

4.2 Conceptual plot of resonant current and inverter output voltage based on energy injection and free oscillation technique. . . . . . . . . . 95

4.3 Resonant current path in 10 operation modes of the single-phase matrix converter. . . . . . . . . . . . . . . 96 
4.4 The simplified digital SMC based controller for the single-phase AC-AC converter. . . . . . . . . . . . . . . . . . 100

4.5 Simulation results on the case study inductive battery charging system: (a) forward power transfer at $3.5 \mathrm{~kW}$ power (Level 1), (b) forward power transfer at $8 \mathrm{~kW}$ power (Level 2), (c) reverse power transfer at $3.3 \mathrm{~kW}$ power (Level 1), (d) reverse power transfer at $7.5 \mathrm{~kW}$ power (Level 2) . . . . . . . . . . . . . . . . . . . 101

4.6 Series-compensated bidirectional IPT system with a single-phase AC-AC $\mathrm{MC}$ as the primary converter and a conventional $\mathrm{H}$-bridge converter as the secondary converter. . . . . . . . . . . . . . . . . 103

4.7 Concept plot of resonant current, energy injection or regeneration signal, grid current (unfiltered) and grid voltage: (a) forward power transfer mode with positive grid voltage, (b) forward power transfer mode with negative grid voltage, (c) reverse power transfer mode with positive grid voltage, $(\mathrm{d})$ reverse power transfer mode with negative grid voltage.

4.8 Resonant current path in the single-phase AC-AC converter in 10 operation modes. . . . . . . . . . . . . . . . . . 105

4.9 The proposed power controller for the primary single-phase AC-AC converter for bidirectional IPT systems. . . . . . . . . . . . . . 107

4.10 Concept plot of the switching signals of the converter $\left(S_{A 1}, S_{A 2}, S_{B 1}, S_{B 2}\right)$ along with the resonant current $\left(i_{r}\right)$, grid voltage $\left(V_{g}\right)$, energy injection/regeneration signal $\left(S_{i n j} / S_{\text {reg }}\right)$, unfiltered grid current $\left(i_{g}\right)$ : (a) forward power transfer mode with positive grid voltage, (b) forward power transfer mode with negative grid voltage, (c) reverse power transfer mode with positive grid voltage, (d) reverse power transfer mode with negative grid voltage. . . . . . . . . . . . . . 108

4.11 The efficiency of the single-phase AC-AC converter as a function of power transfer level and equivalent reflected resistance. . . . . . . . 116

4.12 Simulation results including the grid voltage and current, the energy injection/regeneration signals, the resonant current and the battery charging/discharging current. . . . . . . . . . . . . . . 118

4.13 The simulation results of a G2V connection at the maximum power transfer level $(15 \mathrm{~kW})$ in a full grid voltage cycle showing grid voltage, grid current, battery charging voltage and current. . . . . . . . . . 121

4.14 The efficiency of a single-phase AC-AC converter controlled using the proposed power controller and conventional controller at different power transfer levels calculated based on simulations. . . . . . . . . . 121 
4.15 The IPT system setup comprised of primary and secondary pads, primary AC-AC matrix converter controlled using the proposed power transfer controller, and the secondary converter. . . . . . . . . . . 122

4.16 Experimental test results on the case study IPT system setup including the grid voltage and current (unfiltered), the energy injection/regeneration signals, and the resonant current: (a), (b) level 2 forward power transfer at positive and negative peak grid voltage, (c), (d) level 6 forward power transfer at positive and negative peak grid voltage, (e), (f) level 6 reverse power transfer at positive and negative peak grid voltage, $(\mathrm{g}),(\mathrm{h})$ level 7 reverse power transfer at positive and negative peak grid voltage. . . . . . . . . . . . . . . . 123

5.1 Proposed three-phase ac-ac matrix converter. . . . . . . . . . . . . 128

5.2 A conceptual plot of three-phase input voltages, resonant current and corresponding switching signals of the converter in different modes of operations. . . . . . . . . . . . . . . . 129

5.3 The current path in the proposed converter in eight modes of operation. 133

5.4 The IPT model and its components simulated in MATLAB/Simulink. . 136

5.5 Efficiency of the converter vs. switching frequency $\left(f_{s w}\right)$ and resonant current $\left(i_{r}\right)$, calculated analyticaly. . . . . . . . . . . 142

5.6 Simulation results of the IPT system with $i_{r e f}=282.8$ A. . . . . . . . . 142

5.7 Frequency spectrum of the resonant current shown in Fig. 5.6. . . . . . 143

5.8 The case study IPT system with circular power pads. . . . . . . . . . 145

5.9 Experimental results on the case study IPT system: Output resonant current and corresponding switching signals. . . . . . . . . . . . 145

5.10 Experimental results on the case study IPT system: input voltage $\left(V_{a}\right)$, input current $\left(i_{a}\right)$, and power $\left(P_{a}\right)$ for phase $\mathrm{A}$ and output resonant current $\left(i_{r}\right) \ldots \ldots \ldots \ldots \ldots$. . . . . . . . . . . . . . . . . . . . . . .

5.11 Frequency spectrum of the resonant current shown in Fig. 5.10. . . . . . 147

5.12 A direct three-phase to single-phase AC-AC matrix converter connected to an equivalent RLC circuit representing an IPT system. . . . . . . . . . . . . . . . . 150

5.13 The conceptual plot of the primary resonant current and corresponding output voltage of the converter in energy-injection and freeoscillation modes. . . . . . . . . . . . . . . . . . . 150 
5.14 The proposed simplified digital power controller for the three-phase AC$\mathrm{AC}$ matrix converter designed based on the switching logic given in $(5.28) \ldots \ldots \ldots \ldots 151$

5.15 Simulation results on the proposed AC-AC matrix converter: (a) forward power transfer mode at $68.2 \mathrm{~kW}$ charging power, (b) reverse power transfer mode at $26.4 \mathrm{~kW}$ discharging power, . . . . . . . . . . . 154

5.16 The IPT system setup used for experimental analysis. . . . . . . . . . . 156

5.17 Experimental results on the proposed three-phase matrix converter proto-type: resonant current $\left(i_{r}\right)$, and unfiltered phase A current $\left(i_{a}\right)$, switching signals $S_{A 1}, S_{A 2}$. . . . . . . . . . . 157

6.1 Typical IPT system with a primary self-tuning controller. . . . . . . . . 160

6.2 The magnetic flux density inside the case study IPT system: (a) normal operation, (b) a conductive object is placed on the primary coil. . . . 161

6.3 3D model of the case study IPT system. . . . . . . . . . . . . . 163

6.4 Flux density in the circular power pad. . . . . . . . . . . . . . . 163

6.5 Surface loss density on the sample foreign object. . . . . . . . . . . 164

6.6 Primary self-inductance $\left(L_{p}\right)$, secondary self-inductance $\left(L_{s}\right)$ and mutualinductance $(M)$ versus the location of the conductive object relative to the center of the transmitter. . . . . . . . . . . . . . . . . . 164

6.7 Resonance frequency of the IPT system versus the location of the conductive object respect to the center of the transmitter pad, calculated

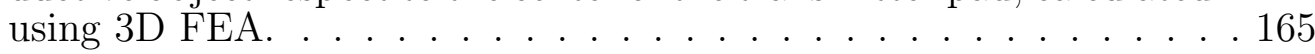

6.8 The proposed primary converter system with self-tuning controller and FOD and a low-power DC source for initial (standby) detection. . . . 165

6.9 Resonance frequency deviation of the IPT system versus the location of the receiver pad relative to the center of the transmitter pad. . . . . 165

6.10 The case study IPT system with a self-tuning controller. . . . . . . . . 168

6.11 Experimental test results on different foreign objects: (a) 5-cent coin with 200 ms detection speed and 49\% detection area, (b) beverage can with $10 \mathrm{~ms}$ detection speed and $82 \%$ detection area. . . . . . . 170

6.12 Detection area versus detection speed calculated based on the experimental test results on the case study IPT system. . . . . . . . . . . . 170

6.13 A typical inductive charging system with a self-tuning controller in the primary side. . . . . . . . . . . . . . . . . 172 
6.14 The proposed proximity detection system based for inductive electric vehicle charging systems. . . . . . . . . . . . . . . . . . . 173

6.15 Magnetic flux lines and field intensity of the wireless charging system at different transmitter and receiver pad center-to-center distances: (a) 3 meters, (b) 2 meters, (c) 1 meter, (d) 0 meter. . . . . . . . . . . . 174

6.16 Self-inductance of the primary and corresponding resonance frequency of the IPT system as function of vehicle alignment respect to the primary power pad. . . . . . . . . . . . 176 


\section{CHAPTER 1}

\section{INTRODUCTION}

Inductive power transfer (IPT) is an emerging technology for transferring power from an energy source to a receiver without any physical contact based on electromagnetic induction. This technology has recently found a wide range of applications in commercial and residential sections such as material handling [1,2], biomedical implants [3,4], transportation systems $[5,6]$ and static [7-9] and dynamic electric vehicle charging systems [10-14]. Using IPT technology wireless power transfer from one system to another across a relatively large distance can be achieved. This is achieved by enabling resonant magnetic induction in two loosely-coupled inductors which have a weak magnetic coupling. Since IPT is unaffected by dust or chemicals and eliminates sparking and the risk of electrical shock, it can be used in hazardous environments. This technology offers high reliability, robustness, high efficiency and provides a clean, safe, and robust way of transferring power. Therefore, IPT has rapidly gained an increasing interest in industrial and commercial sectors.

Contactless electric vehicle (EV) charging based on IPT is a new technology that brings more convenience and safety to the use of EVs. It enables automated

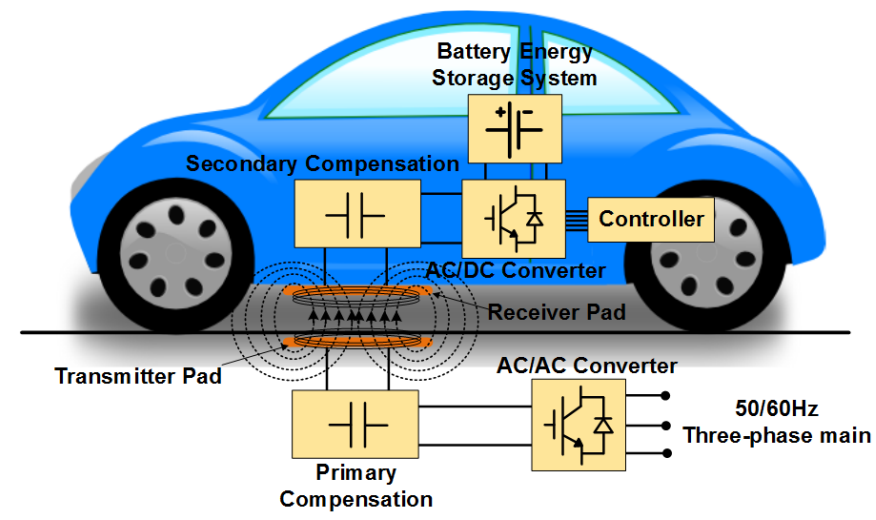

Figure 1.1: A typical contactless electric vehicle charging system based on inductive power transfer. 
charging processes without the need for interaction of the driver by eliminating the charging cables. Contactless EV charging is divided into two main categories: static charging [15-39] and dynamic (in-motion) [5, 11, 16,40-83] charging. In static charging, is aimed to charge EVs using a contactless charger when the EV is parked in a charging station. This is a solution that enables safe, efficient and convenient overnight recharging of EVs [9]. A typical static inductive EV charging system is shown in Fig. 1.1. This system is comprised of a power supply, primary and secondary converters, loosely-coupled magnetic structures (transmitter and receiver structures), and corresponding compensation components.

On the other hand, dynamic contactless EV charging systems based on IPT are designed to provide a wireless power transfer link to the moving EVs on the roadways. This technology can significantly increase the range of EVs by providing continuous wireless power while EVs are moving. Therefore, using dynamic contactless EV charging systems, the required size of the traction batteries in EVs can be reduced. A typical structure of a dynamic contactless EV charging system is shown in Fig. 1.2. This system is composed of multi-conductor underground transmitter tracks, a receiving structure mounted under the EV. In order to achieve a convenient dynamic EV charging, the system should be able to tolerate high levels of misalignments with respect to the underground tracks $[5,12]$.

A roadway IPT system is a safe, robust and clean way of transferring power to EVs with high flexibility, convenience and is unaffected by weather and dirt. Roadway IPT systems have already been proposed for more than two decades [40]. However, due to technological barriers, commercial developments have been slow. A typical roadway inductive power transfer system and its components are shown in Fig. 1.2. This system is composed of primary transmitter tracks, secondary receiver magnetic structures, compensation capacitors, power converters, controllers 


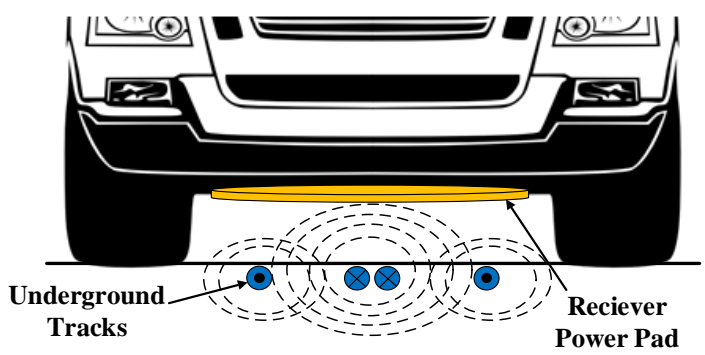

Figure 1.2: Typical structure of a dynamic (roadway) contactless EV charging system EV charging system.

and wireless communication modules. The primary tracks, which are built as loops, are placed underground. Track loops are longer than pickup pads and due to a large air gap, the magnetic coupling coefficient between transmitter and receiver would be lower than 10\% [40]. Many studies have been established to improve the efficiency of the magnetic structures with magnetic materials both in the primary tracks and the secondary pad. But the use of magnetic materials in the primary track structures increases the implementation cost and may not be commercially practical.

\subsection{Existing Challenges in Contactless Electric Vehicle Charg- ing Systems}

In order to make inductive charging systems an acceptable solution that can perform as efficient as conventional plug-in charging systems, they need to be highly optimized for different design measures, such as power transfer efficiency, power density, cost efficiency, and misalignment tolerance. Also, such systems should have a high tolerance to misalignments in order to provide a convenient charging process. Moreover, electromagnetic field (EMF) emissions of such inductive charging units should comply with standards to meet human exposure limitations. In the following sections, these key design challenges are investigated. 


\subsubsection{Power Transfer Efficiency}

The power transfer efficiency of an inductive EV charging system is of key importance and should be comparable to the efficiency of conventional plug-in EV chargers, in order to be considered as an unbeatable solution that brings more convenience to the use of EVs. In a loosely coupled IPT system, due to weak coupling of the coils in an inductive link, it requires a strong magnetic field to be created to deliver high power levels at large ranges. To achieve this, it requires the use of a reactive power compensation circuit, as well as power converters that can generate large currents at high frequencies, often in the $\mathrm{kHz}$ ranges. In order to generate a high-frequency current on the primary side, power converters are employed in IPT systems. A typical configuration of an IPT system is shown in Fig. 1.1. The power source of an IPT system is usually the electric utility $(1 \phi$ or $3 \phi)$ supplying power at $50 / 60 \mathrm{~Hz}$, and for portable applications a dc battery source may be used.

The efficiency of inductive EV charging systems can be improved by increasing the magnetic coupling between the transmitter and receiver structures. This requires increasing the size and volume of the magnetic structures. This increased size and volume, however, not only will compromise the ground clearance of EVs, but also leads to a decrease in the power density and cost efficiency of the inductive charging system. Therefore, a good magnetic structure design should consider a trade-off between power transfer efficiency and power density.

Also, the efficiency of inductive charging systems can be improved by using low loss high-frequency power electronics converters. Specifically, power converters with soft-switching (zero-current switching (ZCS) or zero-voltage switching (ZVS)) operations are of a great importance. Since inductive EV charging systems operate at frequencies as high as $85 \mathrm{kHz}$, the use of soft-switching converters is essential.

Such converters can be designed based on the resonance nature of IPT systems. 
This requires the use of controllers that enable ZCS/ZVS by relying on the resonance nature of inductive charging systems.

\subsubsection{Power Density and Cost Efficiency}

Compared to plug-in chargers, inductive charging systems are composed of a few extra components including transmitter and receiver magnetic structures and compensation networks. The compensation networks are usually embedded inside the receiver and transmitter power converters. This increases the size of the power converters and thereby their power density is decreased. Also, this will increase the cost of the converters. Therefore, power electronics should be optimized for power density and compactness.

In static inductive EV charging systems, the transmitter structure is placed on/under the ground and the receiver is mounted under the EV. The space for the receiver coil under the EV and the admissible weight of the magnetic structures are normally limited, and the weight and volume of the magnetic structure should be considered as a constraint. Therefore, conventional structures may compromise the ground clearance of the EV or require major chassis adjustment. As a result, it is essential for both transmitter and receiver magnetic structures to be optimized for size and volume. However, reducing the size of magnetic structures may compromise other characteristics of the system such as power transfer efficiency and misalignment tolerance. Therefore, a trade-off between different design objectives should be established.

Although IPT systems can achieve efficiencies as high as 95\% at high power transfer levels, they produce power losses in different components of the system including magnetic structures. The power losses and high power density requirements 
together bring about design challenges for IPT systems that require electromagnetic and thermal design considerations [7].

The thermal performance of an inductive charging system should be considered in their design process. Specifically, the thermal performance is of great importance in inductive EV charging systems with high power levels light-duty (up $22 \mathrm{~kW}$ ) and heavy-duty (higher than $22 \mathrm{~kW}$ ) EVs. The thermal performance of an inductive charging system is directly related to the distribution of power losses in the system. The power losses in an IPT system is distributed over the magnetic structure, power electronic converters, and compensation networks on both transmitter and receiver sides. The heat dissipation in the power electronic devices is usually optimized by the use of effective heat sinks along with natural air or forced air convection inside the device. Moreover, the thermal management system inside power electronic converters can be either an open-loop or closed-loop controller. Where in a closedloop thermal management system, the temperature of the device is kept around a reference point.

The losses in magnetic structures are distributed over different key components including the Litz wire coil, ferrite cores, and shielding plates. Magnetic structures in IPT systems are cooled either by natural air or forced air $[84,85]$. The air convection is a process that involves both heat transfer and fluid flow processes in fluids. Also, temperature changes in the coils cause changes in the resistance of the coils in the magnetic structures which can significantly affect the losses generated in the coils. Therefore, the electromagnetic model should also be coupled with the thermal and fluid models. Electromagnetic analysis of IPT systems using finite element analysis (FEA) has been studied in many studies [86-88]. However, multiphysics FEA of IPT systems considering electromagnetic, thermal and fluid models has not been reported. 


\subsubsection{Misalignment Tolerance}

Static inductive EV charging systems are aimed to be implemented in parking sta-

tions and therefore, should be able to tolerate some level of misalignment. An unguided vehicle parking requires a large charging zone for more convenience. High tolerance to horizontal misalignment in an IPT system is essential for a convenient vehicle charging. This tolerance is specified to be between $\pm 100 \mathrm{~mm}$ and $\pm 150 \mathrm{~mm}$ or higher [89]. Thus, the inductive charging systems should be designed in a way that have an acceptable horizontal misalignment tolerance. However, similarly, a magnetic structure with high horizontal misalignment tolerance may not be a costefficient solution. Thus, misalignment tolerance should be considered as one of the design measures for inductive charging systems.

\subsubsection{Electromagnetic Field Emissions}

The electromagnetic field (EMF) emissions of inductive charging system for lightduty EVs should comply with SAE TIR J2954 [90] standard. This standard is based on human exposure limitation as defined by the International Commission on NonIonizing Radiation Protection (ICNIRP) [91]. Therefore, EMF limitations should be considered as an important constraint in the design of magnetic structures.

\subsubsection{Foreign Object Detection}

In a loosely-coupled IPT system, the coupling between the transmitter and receiver coils is poor. Therefore, compensation capacitors are used to form a resonant circuit with the self-inductance of the primary, to generate large resonant currents which are essential for transferring power between primary and secondary circuits. Also, to reduce the size of the coils and compensation capacitors and increase the power 
transfer efficiency, IPT systems usually operate at high resonance frequencies up to $90 \mathrm{kHz}$ [90]. Therefore, in a loosely coupled IPT system, high-frequency highmagnitude magnetic fields will be generated in the power transfer area. The highfrequency magnetic fields can induce intense eddy-currents on conductive objects, and generate heat which can lead to a significant temperature rise in the object [92]. As a result, conductive objects should not be exposed to the magnetic fields generated by the IPT system to avoid efficiency reduction and eliminate the risk of fire $[93,94]$. Consequently, a conductive foreign object detection (FOD) mechanism in IPT systems with large air gaps is essential.

Different FOD methods for IPT systems have been proposed, which can be categorized into two major types: sensor-based and sensorless detection methods. The sensor-based FOD methods include distance detection [95], light intensity monitoring [96], image processing [97], thermal sensors [98, 99], and magnetic field sensors [100]. Sensorless FOD methods are designed based on measurement, estimation, and monitoring of different parameters in IPT systems. These parameters include induced voltage [101], capacitance [102], power loss [103,104], and energy decay time [105].

\section{$1.2 \quad$ Literature Review}

In this Section, the background work and literature review of inductive EV charging systems are presented. 


\subsubsection{Magnetic Structures of Inductive Electric Vehicle Charg- ing Systems}

Magnetic structures play a key role in the performance of inductive EV charging systems. These structures should be optimized for different design objectives and ensure the seamless operation of the inductive charging system at any operating condition. Specifically, the magnetic structures should be optimized to have a high power transfer efficiency and high power density and cost efficiency. Further, these structures should be able to allow high tolerance to misalignments to provide more convenience for vehicle alignment. However, these design objectives are incommensurable. For example, a high power density design may compromise the power transfer efficiency and misalignment tolerance or, a cost-efficient design will have a reduced efficiency and misalignment tolerance. Therefore, trade-offs between different design objectives should be considered in the design of magnetic structures.

The design of magnetic structures involves multiple constraints. The stray electromagnetic field (EMF) generated by an inductive charging system should remain within the limits as defined by standards such as SAE J2954 for light-duty inductive EV charging systems [90] which complies with the International Commission

on Non-Ionizing Radiation Protection (ICNIRP) [91]. Also, the power transfer efficiency of the system should remain above $85 \%$ and $80 \%$ in aligned and offset positions, respectively. Further, temperature rises which are caused due to the power losses in magnetic structures should be kept as low as possible and therefore should be considered as a critical design constraint. The addition of these constraints to the trade-offs between different design objectives further increases the complexity of the design optimization problem. 
Various magnetic structures are proposed for both static and dynamic inductive EV charging systems. The magnetic structure designed for small air gaps include U-cores [106, 107], E-cores [107, 108], S-cores [108], pot-cores [109,110], and ferrite discs or plates [111]. Because of the use of large ferrite cores in these structures, they are relatively bulky, expensive, and fragile.

On the other hand, planar magnetic structures are thin, light and do not compromise the ground clearance of the EVs. These structures include, circular $[9,112,113]$, unipolar rectangular [84], bipolar double-D (DD) [11,84], bipolar double-D Quadrature (DDQ) [114], and tripolar [115]. These structures are all recommended by SAE J2954 [90]. The design optimization of these magnetic structures is investigated in different studies. In [9], design and optimization of circular magnetic structures are discussed in detail. In [84,89], multi-objective optimization of magnetic structures of inductive EV charging systems considering power transfer efficiency, power density and stray magnetic field as objective functions are presented. However, a study that provides a generalized systematic approach for the design optimization of magnetic structures in inductive charging systems has not been reported in the literature.

\subsubsection{Power Converter Topologies in Inductive Power Trans-}

\section{fer Systems}

In a loosely-coupled IPT system, the inductive link requires a strong magnetic field to be created to deliver high power levels at large air gaps due to the weak coupling of the coils. To achieve this, this technology requires the use of power converters that can generate large high-frequency currents, often in the kilohertz range $(10-85 \mathrm{kHz})$. In order to generate a high-frequency current on the primary side, specific power converters are employed in IPT systems. Power converters play a key role in the performance of IPT systems. Recent developments in IPT systems have heightened 
the need for high-power, reliable and efficient converters. Normally, these converters take 50/60 Hz current from the $\mathrm{AC}$ mains and convert to high-frequency using a two-stage AC-DC-AC power conversion. The power source of an IPT system is usually the electric utility (single-phase or three-phase) supplying power at 50/60 $\mathrm{Hz}$.

Voltage-source inverters (VSI) based on pulse-width modulation (PWM) with a front-end rectifier have become the preferred choice for most practical applications [116]. This is mainly due to their simple topology and low cost. On the other hand, this two-stage topology has low-frequency harmonics on the de link and the ac input line, which requires the use of very bulky short-life electrolytic capacitors for the dc link and a large low-pass filter at the output [116]. Several topologies have been proposed to solve the problems of the traditional ac-dc-ac power converters [117-119]. Matrix converters are the main alternatives for two-stage converters that can convert energy directly from an ac source to a load with different frequency and amplitude without any energy storage elements [120]. These converters have the advantages of the simple and compact topology, bidirectional power flow capability, high-quality input-current waveforms, and adjustable input power factor independent of the load [116,120-123]. Particularly, different converter topologies have been proposed for IPT applications [124-132]. A simple, compact and highly efficient single-phase matrix converter for IPT applications is presented in [124]. The energy injection and free oscillation control strategy is applied to the topology. However, this matrix converter suffers from current sags around input ac voltage zero-crossings.

The magnetic structures are inherently capable of transferring power in either direction. However, in order to enable bidirectional power transfer in IPT systems, the use of bidirectional AC-AC power converters is essential. Conventionally, four- 


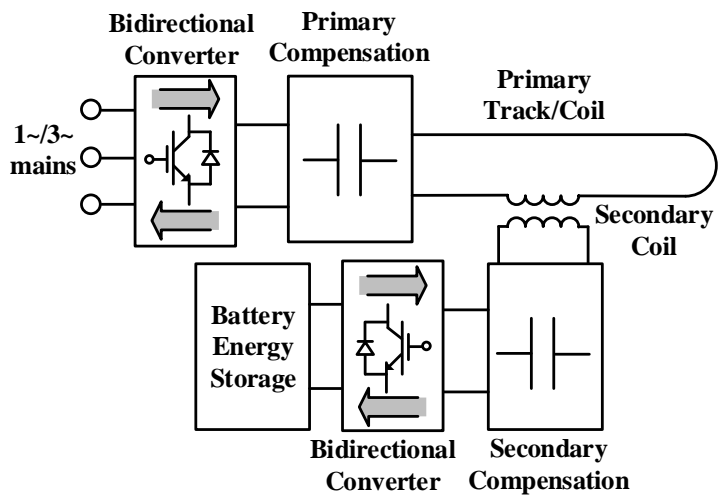

Figure 1.3: The structure of a bidirectional IPT system.

quadrant two-stage AC-AC converters are proposed for bidirectional IPT systems [130]. However, in recent years there has been an increasing interest in matrix converters (MCs). MCs are used to convert AC mains inputs to an AC output with a different frequency and amplitude directly without any intermediate conversion stage. In MC topologies the bulky energy storage elements are eliminated and thereby, they have high power density and are more reliable. Specifically, threephase to single-phase and single-phase to single-phase matrix converters are of great interest in IPT systems. These types of converters have been successfully employed with a reduced number of elements and improved efficiency [124,133]. Since MCs are inherently bidirectional, they can be used to regenerate power from an IPT system back into the source and as a result, they can be used for inductive EV charging/discharging applications where G2V and V2G connections can be realized.

In [124], a half-wave single-phase MC for inductive power transfer systems which is comprised of two bidirectional switches is proposed. This converter, however, can only inject energy to the IPT systems in positive or negative half-cycle depending on the input voltage sign. In other words, half of the resonant current half-cycles are free-oscillation modes which significantly reduces the maximum extractable power from AC mains. 
Bidirectional IPT systems have been studied in [125,134-145]. The magnetic structures in IPT systems are inherently capable of transferring power in either direction. However, in order to enable bidirectional power transfer in IPT systems, the use of bidirectional power converters is essential. Specifically, a bidirectional $\mathrm{AC}-\mathrm{AC}$ converter is essential in the primary side, as an interface between $\mathrm{AC}$ mains and the IPT system. Conventionally, two-stage AC-AC converters are used for IPT systems including H-bridge converters [135], current source converters [125, 134], z-source inverters [146], multilevel inverters [130]. However, in recent years there has been an increasing interest in matrix converters (MCs) $[124,133,147,148]$. MCs are used to directly convert AC mains inputs to a high-frequency AC (HFAC) output without any intermediate conversion stage. In MCs, the bulky energy storage elements are eliminated and thereby, they have high power density and are more reliable. Specifically, three-phase to single-phase and single-phase to single-phase matrix converters are of great interest in IPT systems. These types of converters have been successfully employed with a reduced number of elements and improved efficiency [124,133]. Resonant MCs have been of great interest in many applications. In [148], a single-phase AC-AC resonant converter with soft-switching operation sliding-mode control is introduced.

In [147], a single-phase MC based on a modulation technique for bidirectional IPT systems is proposed. The converter enables attenuation of the harmonic distortions in the grid current. However, the converter is hard-switched, which leads to a lower efficiency and reliability and requires complex commutation techniques. Quantum resonant converters which are introduced in 1989 [149] have found an increasing interest for IPT systems. In [124], a half-bridge single-phase quantum MC comprised of two bidirectional switches is proposed. Due to the reduced number of switches in the converter, energy injection to the resonant tank can be only car- 
ried out in half of the resonant current half-cycles. Therefore, the maximum power transfer that can be achieved by the converter is limited.

\subsubsection{Control Methods of Inductive Power Transfer Systems}

Different control methods for IPT systems and resonant converters have been proposed. These methods include power-frequency control $[135,150]$, phase-shift and frequency control [151,152], load detection [153], power flow control [17], and sliding mode control (SMC) [148,154-156]. The control methods can have a self-tuning capability $[157,158]$. This feature makes these controllers suitable for dynamic IPT applications, where the resonance frequency of the system may have small variations due to load variations on the receiver side. Furthermore, it can find many applications such as energy encryption [159], where the IPT system has a variable

resonance frequency with the use of variable compensation capacitors. Specifically, a very effective method which is widely used as control technique in resonant power electronic converters is energy-injection free-oscillation method. This control technique has been successfully employed in many studies $[124,160]$. This method can be employed in different converter topologies such as two-stage AC/DC/AC converters [160] and matrix converters [124]. Using this method, the resonant current can be regulated by controlling the energy transfer rate which is injected to the primary LC tank. This is achieved by constantly switching between two free-oscillation and energy-injection operation modes of the converter.

Power flow control plays a crucial role in the IPT system's optimal operation, which can in turn be attained by controlling the transferred power to either the transmitter or the receiver, or both [17]. In the literature, several techniques for power flow control for IPT systems exist, including resonance frequency control 
[161], power-frequency control [128,135,150], phase-shift control [151,152,157], load detection [153], reactive power control [17], and sliding mode control (SMC) [148, 154]. Tuning the control parameters and handling the system variability can be made possible by modifying the control methods to be adaptive with self-tuning capability $[158,162]$. The self-tuning capability of such controllers can significantly enhance the performance and interoperability of IPT systems.

Soft-switching operations in power converters can significantly improve the efficiency and reduce their electromagnetic field interference (EMI) [163-169]. Since IPT systems for electric vehicle applications usually operate at frequencies ranging between 20 and $85 \mathrm{kHz}$, the performance of the power converters, considerably affect their performance. Thus, by ensuring soft-switching operations of the converter, the controllers can greatly improve the performance of the system. One method capable of effective power flow control in IPT systems is the energy-injection free-oscillation. This technique, which enables soft-switching operations, has been successfully applied in many studies $[124,133,160,162]$.

Conventionally, pulse-width modulation (PWM) based power electronic converters are used in IPT systems and desired outputs are achieved by controlling the frequency and duty-cycle of the PWM signal [130,137,170,171]. In [162], a control method based on a variable frequency control technique is proposed for inductive EV charging systems. This method achieves multiple power transfer levels in a fullbridge DC/AC converter and is designed to reduce the frequency of energy injection (rather than changing the duty-cycle of a PWM signal) without compromising the resonance behavior of the IPT system. The developed controller is open-loop and provides multiple power transfer levels.

The resonance frequency in an IPT system can slightly deviate from its nominal point due to changes in operating conditions. The deviation of the switching 
operations from the resonance point can dramatically affect the performance of the system. Therefore, constant switching adjustment to the resonance frequency variations is essential to ensure high power transfer efficiency in the system. The resonance frequency tracking capability of the controller enables synchronization of the switching operation of the converters with the resonant current to achieve maximum power transfer with high efficiency [157, 162,172,173].

In order for an IPT system to operate at the proper operating point with high performance, the use of controllers for power transfer regulation, resonant current regulation, switching frequency tuning, etc. are of a great importance. Different control strategies for resonant IPT systems have been proposed, such as powerfrequency control $[135,150]$, frequency and phase-shift control [151], load detection [153], power flow control [17], and sliding mode control [154]. The control methods can have a self-tuning capability $[157,162,174]$. This feature makes these controllers suitable for dynamic IPT applications, where the resonance frequency of the system may have small variations due to load variations on the receiver side. One of the effective methods for controlling the transferred power in an IPT system is the amplitude modulation of the resonant current based on energy-injection technique. This control technique can be designed for a wide range of converter topologies including two-stage $\mathrm{AC} / \mathrm{DC} / \mathrm{AC}$ and single-stage matrix converters. This technique has been successfully employed in single-phase and three-phase matrix converters to effectively regulate the resonant current $[124,133,175]$.

The requirements for light-duty static inductive EV charging systems are established in SAE TIR J2954 Standard [90]. This standard defines four inductive charging levels as $3.7 \mathrm{~kW}, 7.7 \mathrm{~kW}, 11 \mathrm{~kW}$ and $22 \mathrm{~kW}$ for light-duty EVs with a minimum power transfer efficiency of $85 \%$. Also, the standard requires the inductive charging system to have a single nominal operating frequency of $85 \mathrm{kHz}$. Although 
the requirements for static inductive charging for heavy-duty EVs are not defined in the standard, it is successfully implemented in many studies $[14,17,84]$.

Similar to conductive charging systems, inductive EV charging systems are capable of bidirectional power transfer which enables V2G, G2V, V2I and, V2V connections through the IPT system. Since such inductive connections can be easily and quickly established at different geographical locations of the power system, they can make the power system more complex and dynamically varying.

\subsection{Research Objectives and Original Contributions}

The aim of this research is to tackle the existing challenges in inductive electric vehicle charging systems. Specifically, the following research areas are investigated:

\section{Generalized Physics-Based Multi-Objective Design Optimization of Magnetic Structures for Inductive Charging Systems}

The aim of this objective is to introduce a generalized approach for design optimization of magnetic structures for inductive charging systems that addresses the trade-off problem between several key objectives including efficiency, power density, misalignment tolerance, and cost efficiency considering critical constraints. The design problem is formulated as a constrained multi-objective optimization problem (MOP). The MOP is then solved using a genetic algorithm (GA) which is coupled to a physics-based model of the inductive charging system. The physics model is a 3D parametric hybrid electromagnetic and thermal model which is solved using finite element analysis (FEA) and is coupled to the circuit model. The proposed optimization method complies with SAE J2954 standard for inductive charging systems in terms of interoperability and electromagnetic compatibility (EMC). Using the proposed method, 
design optimization of multiple planar topologies are investigated. Specifically, a $3.7 \mathrm{~kW}$ inductive charging system is investigated as a case study and a prototype is built to validate the optimization results. The results show that the proposed generalized multi-objective optimization method provides a holistic solution for obtaining the magnetic design parameters of inductive EV charging systems. Using the proposed method, the magnetic structures can be effectively designed by prioritizing the objectives. Using the developed design methodology, a $3.7 \mathrm{~kW}$ inductive charging system with square magnetic structures is investigated as a case study and a prototype is built to validate the optimization results. The developed prototype achieves $93.65 \%$ efficiency (DC-to-DC) and a power density of $1.65 \mathrm{~kW} / \mathrm{dm}^{3}$.

\section{Design and Development of Self-Tuning Controllers for Inductive Power Transfer Systems}

In this objective, self-tuning controllers for power transfer regulation in inductive power transfer (IPT) systems are proposed. The proposed controllers enable power transfer regulation around a user-defined reference power level. The converter's efficiency is improved by constantly tuning the switching operations to the resonant current zero-crossing points, thereby achieving the soft-switching operations reducing the power losses and electromagnetic interference (EMI) the power converters. The self-tuning capability makes it suitable for dynamic IPT systems with uncertain loads and fluctuating resonance frequency. High operating frequencies can be achieved using the proposed simplified digital circuit designs for the controllers which deliver a low total propagation delay. Bidirectional power transfer can be enabled by using the proposed controllers on both transmitter as well as receiver sides. In the reverse power flow mode, the primary converter operates as a rectifier and the 
power transfer is controlled through the secondary converter using the proposed controllers. The performance of the proposed controllers is analyzed using MATLAB/Simulink, and the results are presented detail. Finally, the proposed controllers are implemented experimentally and their performance is evaluated in a case study IPT system. The experimental and simulation results conform to each other and show that the proposed converter can effectively regulate the power transfer with an improved efficiency.

It is shown that the proposed controllers can effectively control the power transfer level in IPT systems, which uses different predefined energy injection levels to maintain user-defined power transfer levels. The controllers' selftuning functionality renders it suitable for dynamic IPT applications where the resonance frequency and the load might vary. The proposed controller can also significantly improve efficiency and reduce the EMI by enabling softswitching operations. The controller is further designed using a simplified digital circuit that can lower costs by eliminating the need for availing more expensive solutions, such as DSP and FPGA. Therefore, the controllers can operate at high frequencies. These salient features of the controllers including low cost and high efficiency, therefore, make it a powerful alternative that can be applied to a wide variety of IPT systems. Several simplified digital controllers are developed and experimentally implemented. The controllers are implemented without the use of DSP/FPGA solutions. Experimental tests show that of the developed simplified controllers can effectively regulate the power transfer around the desired value. Moreover, the experiments show that compared to conventional converters, the developed converters can achieve $4 \%$ higher efficiency at low power levels. 


\section{Development of Direct AC-AC Matrix Converter Topologies Induc- tive Power Transfer Systems}

Direct single-phase and three-phase AC-AC matrix converters for inductive power transfer (IPT) systems with soft-switching operation are investigated in this objective. The proposed topologies are expected to have a high reliability and extended lifetime due to the soft-switching operation and elimination of short life electrolytic capacitors. The soft-switching operations will also reduce switching stress, switching loss and electromagnetic interference (EMI) of the converters. Quantum energy injection/regeneration principle is used to design a simplified digital power controller that enables the converter to establish bidirectional power transfer between the IPT system and single-phase AC mains at a desired power level. The simplified controller can be implemented using basic logic circuit components, without the need for DSP/FPGA platforms, thereby reducing the complexity and the implementation cost. The converters benefit from resonance frequency tracking capability for the synchronization of switching operations of the converters with the resonant current which makes it ideal for dynamic IPT systems. The converters are specifically suitable for establishing grid-to-vehicle (G2V) and vehicle-togrid (V2G) connections through inductive electric vehicle charging/discharging systems. The proposed converters are analyzed theoretically, are simulated in MATLAB/Simulink, and finally are verified experimentally on a case study

IPT system. The results show that the proposed matrix converters can effectively establish bidirectional power transfer at different power levels with soft-switching operation and resonance frequency tracking capability.

Specifically, a direct three-phase AC-AC matrix converter with reduced number of switches (only seven) is developed and built. Due to reduced switch- 
ing elements the converter can achieve high reliability, efficiency and costefficiency. The converter operates in eight modes operation modes, which are described in detail. A variable frequency control strategy based on quantum energy injection/regeneration principle is employed to regulate the resonant current, the resonant voltage, and the output power. With the use of the proposed converter as the primary converter, simulation analysis and experimental implementations on a case study IPT system, show that the current regulation control method can fully regulate the output current and output power around user-defined reference values. Thus, it is suitable for dynamic IPT applications, where the system has inherent variations. It is shown that the developed converters can achieve efficiencies as high as $98.54 \%$ at high power levels and outperform conventional two-stage converters.

\section{Further Applications of Self-Tuning Power Controllers in Inductive EV Charging Systems}

The introduced self-tuning controllers can find many applications in inductive EV charging system. In this objective, a few applications that utilize the power transfer control along with resonance frequency tracking capabilities of the developed controllers are introduced. These applications include, sensorless conductive foreign object detection (FOD) and sensorless vehicle proximity detection.

It is shown that the proposed FOD method based on the resonance frequency deviation provides a fast and effective technique for detection of conductive foreign objects in IPT systems. By the use of self-tuning controllers, the need for detection sensors is eliminated, which as a result simplifies the implementation of the method. The proposed technique can be implemented for initial 
(standby) detection at low-power and online detection at full-power. Experimental results on a case study IPT system show that the proposed method is effective in detecting conductive objects and in initial detection mode, it can detect a 5-cent coin in the power transfer area in about $200 \mathrm{~ms}$. However, comparisons between the effect of vehicle variations and conductive foreign objects on the resonance frequency deviation show that online detection mode would not be effective for detection of such small objects. The detection of larger objects is achieved in both initial detection and online detection modes with much higher speed and larger detection area. Furthermore, it is shown that by reducing the detection speed, the detection area is significantly increased and much smaller conductive objects can be detected. The resonance frequency deviations are proportional to the size and shape of the objects and in fact can be used as a measure for the size of the object.

A vehicle proximity detection method is proposed which provides a simple and effective mechanism which can be used in inductive charging stations to enable automated inductive charging process. Since the transmitter magnetic structure is used as the detection coil, the proposed method can be implemented in any inductive charging system and requires minor system modifications. The resonance frequency of the inductive charging system is used as a measure for vehicle detection. The resonance frequency tracking capability is enabled using self-tuning controllers for IPT systems which have a relatively simple design. The simulations results show that the vehicle can be detected in about 1.5 meters from the perfect alignment and once the resonance frequency recovers to its original value indicates that the vehicle is perfectly aligned and therefore the charging process can be initiated. 


\section{CHAPTER 2}

\section{GENERALIZED PHYSICS-BASED MULTI-OBJECTIVE DESIGN OPTIMIZATION OF MAGNETIC STRUCTURES FOR INDUCTIVE CHARGING SYSTEMS}

Magnetic structures in inductive electric vehicle (EV) charging systems should be designed to achieve high power transfer efficiency and high power density. Also, these structures should be able to provide high tolerance to misalignments for more convenient vehicle alignment. Therefore, magnetic structures should achieve multiple design objectives simultaneously which makes the design process challenging. The aim of this study is to introduce a generalized approach for design optimization of magnetic structures for inductive charging systems that addresses the trade-off problem between several key objectives including efficiency, power density, misalignment tolerance, and cost efficiency considering critical constraints. The design problem is formulated as a constrained multi-objective optimization problem (MOP). The MOP is then solved using a genetic algorithm (GA) which is coupled to a physics-based model of the inductive charging system. The physics model is a 3D parametric hybrid electromagnetic and thermal model which is solved using finite element analysis (FEA) and is coupled to the circuit model. The proposed optimization method complies with SAE J2954 standard for inductive charging systems in terms of interoperability and electromagnetic compatibility (EMC). A $3.7 \mathrm{~kW}$ inductive charging system is investigated as a case study and a prototype is built to validate the optimization results. 


\subsection{Multi-Objective Design Optimization of Square Mag- netic Structures for Static Inductive Charging Systems}

In this section, a generalized approach for the multi-objective design optimization of magnetic structures is presented. The proposed approach incorporates several objectives including power transfer efficiency, horizontal misalignment tolerance, power density and specific cost. Also, design constraints including the ones defined by SAE J2954 standard are also included. The design optimization is formulated as a constrained multi-objective optimization problem (MOP). A Genetic Algorithm coupled with a hybrid analytical-numerical formulation is developed in order to solve the MOP. The MOP is then solved using genetic algorithm (GA) which is coupled to a physics-based circuit model of the inductive charging system. The physicsbased model is a 3D parametric hybrid electromagnetic and thermal model which is solved using finite element analysis (FEA) and is coupled to the circuit model. The physics model is employed to precisely calculate circuit parameters such as magnetic couplings, equivalent resistance of the coils, magnetic cores, and other structural components. In this work, COMSOL Multiphysics is employed to perform the 3D FEAs. The proposed optimization method complies with SAE J2954 standard for inductive charging systems in terms of interoperability and electromagnetic compatibility (EMC). A 3.7kW (class WPT1/Z1 of SAE J2954) inductive charging system is investigated as a case study and optimized magnetic structures are presented for several design scenarios. A prototype is built based on the optimized design parameters for maximum power density. The experimental results show that the proposed method effectively results in the desired optimized magnetic structures with high accuracy. 


\subsubsection{Derivation of Equivalent Circuit Model of an Induc- tive Charging System}

In order to interpret the effect of magnetic structure design on the performance of inductive charging systems, a detailed circuit analysis is essential. The circuit diagram of a typical series compensated inductive charging system is presented in Fig. 2.1(a). The system is composed of a primary rectifier, primary $\mathrm{DC} / \mathrm{AC}$ converter, magnetic structure equivalent model $\left(L_{1}, L_{2}, M\right)$, series compensation capacitors $\left(C_{1}, C_{2}\right)$, and secondary battery charger (AC/DC converter, traction battery).

The equivalent circuit of a series-compensated inductive battery charger, can be derived using fundamental harmonic approximation (FHA) [176]. Since in IPT systems, the output voltage of the primary DC/AC converter has a fundamental

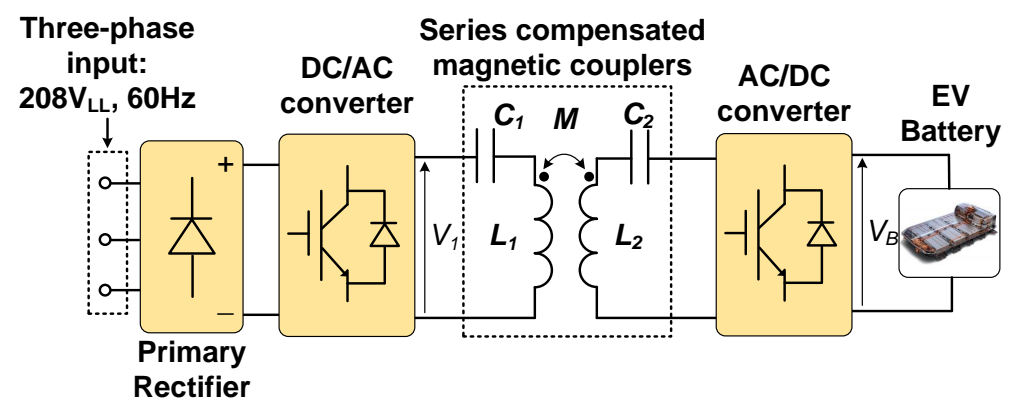

(a)

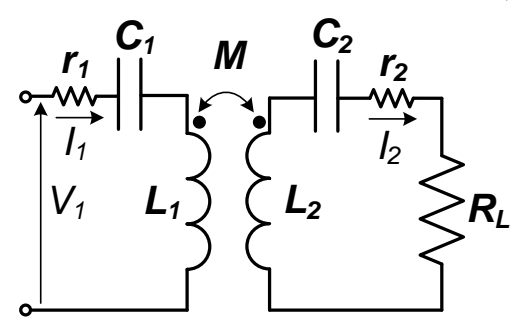

(b)

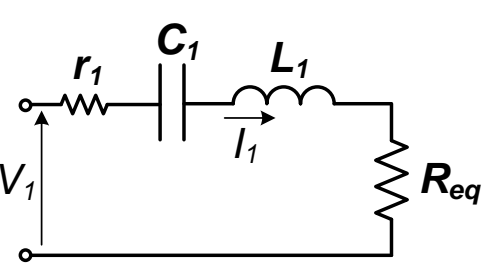

(c)

Figure 2.1: The circuit diagram of series compensated inductive charging systems (a) complete model including primary and secondary power converters, (b) simplified fundamental harmonic model, (c) primary side model. 
harmonic component exactly at the resonance frequency, FHA can be applied in order to model all the voltages and currents as sinusoidal at the resonance frequency.

\subsubsection{Equivalent Model of a Full-Wave Battery Charger}

A battery charger has inherently nonlinear characteristics. However, a full-wave battery charger can be modeled using FHA method. Using FHA, tA battery charger has inherently nonlinear characteristics. However, a full-wave battery charger in a resonant circuit can be modeled using FHA method. Using FHA, the battery charger on the secondary side of an inductive charging system can be modeled as a variable equivalent resistance. The equivalent ac resistance of a full-bridge rectifier $\left(R_{L}\right)$ feeding a DC load $R_{B}$ can be expressed as [176],

$$
R_{L}=\frac{8}{\pi^{2}} R_{B}
$$

The equivalent DC resistance of the battery can be written as $R_{B}=V_{B} / I_{B}$. Therefore, (2.1) can rewritten as,

$$
R_{L}=\frac{8 V_{B}}{\pi^{2} I_{B}}
$$

Also, it can be easily shown that in a full-wave rectifier the relation between DC current $\left(I_{d c}\right)$ and secondary RMS AC current $\left(I_{2}\right)$ is as follows,

$$
I_{B}=\frac{2 \sqrt{2}}{\pi} I_{2}
$$

Using (2.2) and (2.3), the equivalent $\mathrm{AC}$ resistance can be rewritten as,

$$
R_{L}=\frac{2 \sqrt{2}}{\pi} \frac{V_{B}}{I_{2}}
$$

Equation (2.4) gives the equivalent load of a battery charger at the secondary as function of ac current and battery voltage. 


\subsubsection{Equivalent Circuit of Series Compensated Inductive Battery Charger}

Derivation of Equivalent Circuit Model of an Inductive Charging Systems The equivalent circuit of the inductive charging system is shown in Fig. 2.1(b) where, $V_{1}$ and $I_{1}$ are the voltage and current outputs of the primary converter respectively which are applied to the primary circuit, $I_{2}$ is the secondary current, $r_{1}$ and $r_{2}$ are the equivalent resistances of the primary and secondary respectively which incorporate resistance of power converters, compensation capacitors, coils, magnetic cores and shielding plates (Section 2.1.2). This circuit can be further simplified by reflecting the battery charging load to the primary side as shown in Fig. 2.1(c). The secondary current $I_{2}$ then can be calculated as,

$$
I_{2}=\frac{\omega M I_{1}-\frac{2 \sqrt{2}}{\pi} V_{B}}{r_{2}}
$$

In a series-series compensated IPT system which operates at the resonance frequency, the reflected resistance to the primary can be expressed as [7],

$$
R_{e q}=\frac{\omega^{2} M^{2}}{r_{2}+R_{L}}
$$

Using (2.4) and (2.5), (2.6) can be rewritten as,

$$
R_{e q}=\frac{\omega M\left(\pi \omega M I_{1}-2 \sqrt{2} V_{B}\right)}{\pi r_{2} I_{1}}
$$

By applying KVL to the equivalent circuit of the primary, the following can be derived,

$$
V_{1}=\left(r_{1}+R_{e q}\right) I_{1}
$$

where $V_{1}$ is the main harmonic component of the input voltage applied to the primary. Using (2.7) and (2.8), the primary and secondary currents $I_{1}, I_{2}$ can be written as,

$$
I_{1}=\frac{\pi r_{2} V_{1}+2 \sqrt{2} \omega M V_{B}}{\pi\left(r_{1} r_{2}+\omega^{2} M^{2}\right)}
$$




$$
I_{2}=\frac{\pi \omega M V_{1}-2 \sqrt{2} r_{1} V_{B}}{\pi\left(r_{1} r_{2}+\omega^{2} M^{2}\right)}
$$

\subsubsection{Inductive Charging System Efficiency}

The power transfer efficiency can be calculated as,

$$
\eta=\frac{R_{B} I_{2}^{2}}{\left(r_{1}+R_{e q}\right) I_{1}^{2}}
$$

Using (2.9) and (2.10), $\eta$ can be expressed as,

$$
\eta=\frac{2 V_{B}\left(\sqrt{2} \pi \omega M V_{1}-4 r_{1} V_{B}\right)}{\pi V_{1}\left(\pi r_{2} V_{1}+2 \sqrt{2} \omega M V_{B}\right)}
$$

\subsubsection{Optimal Input Voltage}

The input voltage of the IPT system $\left(V_{1}\right)$ can be controlled by the primary DC/AC converter. The optimal input voltage at which maximum power transfer efficiency occurs $\left(V_{\text {opt }}\right)$ can be found by solving the following equation for $V_{1}$,

$$
\frac{\partial \eta}{\partial V_{1}}=0
$$

Using (2.12), $V_{\text {opt }}$ can be simplified as,

$$
V_{\text {opt }}=\frac{2 \sqrt{2} V_{B}\left(r_{1} r_{2}+\sqrt{r_{1} r_{2}\left(r_{1} r_{2}+\omega^{2} M^{2}\right)}\right)}{\pi \omega M r_{2}}
$$

By substituting (2.14) in (2.12), the corresponding efficiency $\eta_{\max }$ is derived as,

$$
\eta_{\max }=1-\frac{2 \sqrt{r_{1} r_{2}\left(r_{1} r_{2}+\omega^{2} M^{2}\right)}-2 r_{1} r_{2}}{\omega^{2} M^{2}}
$$

To further simplify (2.15), the following factor is defined as,

$$
Q^{2}=\frac{\omega^{2} M^{2}}{r_{1} r_{2}}
$$




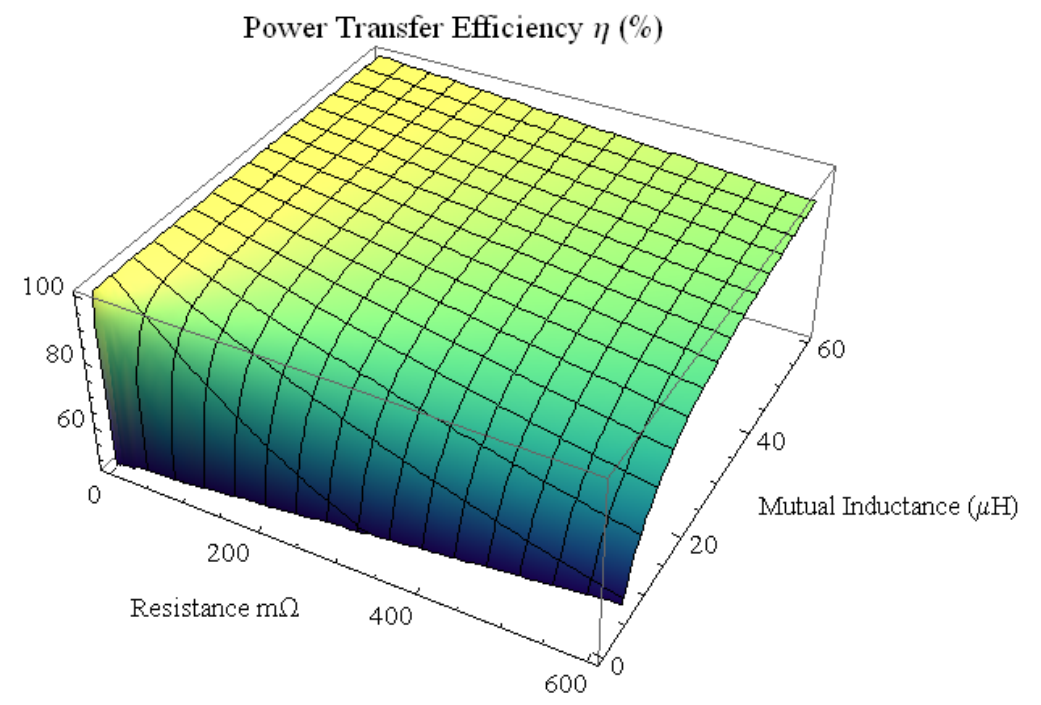

Figure 2.2: Power transfer efficiency $(\eta)$ of an inductive charging system as a function of resistance $r$ and mutual inductance $M$.

where $Q$ is a factor that represents both the level of magnetic coupling and coil quality in primary and secondary magnetic structures. Using (2.16), (2.15) can be simplified as,

$$
\eta_{\max }=1-\frac{2 \sqrt{1+Q^{2}}-2}{Q^{2}}
$$

where the quality factor $Q$ can be calculated using the physics-based model for $M$, $r_{1}$ and $r_{2}$. In Fig. 2.2, the plot of maximum power transfer efficiency as a function $M$ and $r_{1} r_{2}$ is presented. This plot shows that efficiency increases when $M$ increases or when $r_{1} r_{2}$ decreases. This can be mathematically proved by taking the derivative of $\eta_{\max }$ respect to $Q$ as,

$$
\frac{d \eta_{\max }}{d Q}=\frac{2 Q^{2}-2 \sqrt{1+Q^{2}}+4}{Q^{3} \sqrt{1+Q^{2}}}
$$

Based on (2.18), it can be easily shown that for any $Q>0$, we have $\frac{d \eta_{\max }}{d Q}>$ 0. Therefore, $\eta_{\max }$ is a strictly increasing function of $Q$. As a result, achieving maximum $Q$ guarantees maximum power transfer efficiency of the system. 


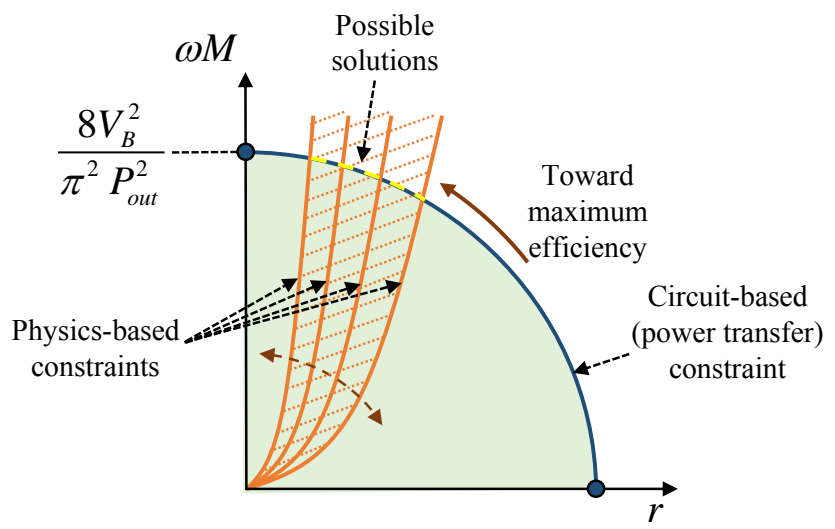

Figure 2.3: Representation of circuit-based (power transfer) and physics-based constraints in $\omega M-r$ coordinates.

\subsubsection{Output Power Constraint}

The power output of an inductive charging system can be expressed as $P_{\text {out }}=R_{L} I_{2}^{2}$. Using (2.4) and (2.10), $P_{\text {out }}$ can be rewritten as,

$$
P_{\text {out }}=\frac{8 r_{1} V_{B}^{2}}{\pi^{2} \sqrt{r_{1} r_{2}\left(r_{1} r_{2}+\omega^{2} M^{2}\right)}}
$$

Equation (2.19) expresses the relationship between output power and mutual inductance and equivalent primary and secondary resistances. In the case that primary and secondary structures are identical, it can be assumed $r_{1}=r_{2}=r$,

$$
P_{\text {out }}=\frac{8 V_{B}^{2}}{\pi^{2} \sqrt{r^{2}+\omega^{2} M^{2}}}
$$

Fig. 2.3 conceptually shows the circuit-based (output power) and physics-based design constraints. Based on (2.20), the circuit-based constrained is a quadrant in $\omega M-r$ coordinates with a radius of $\frac{8 V_{B}^{2}}{\pi^{2} P_{\text {out }}}$.

Inductive charging systems are designed to achieve $Q^{2}>>1$. Therefore, according to $(2.16)$ we have $\omega^{2} M^{2}>>r^{2}$. Thereby, (2.20) can be approximated as $P_{\text {out }}=\frac{8 V_{B}^{2}}{\pi^{2} \omega M}$. Thus, the required mutual-inductance $M_{0}$ can be expressed as,

$$
M_{0}=\frac{8 V_{B}^{2}}{\pi^{2} \omega P_{\text {out }}}
$$




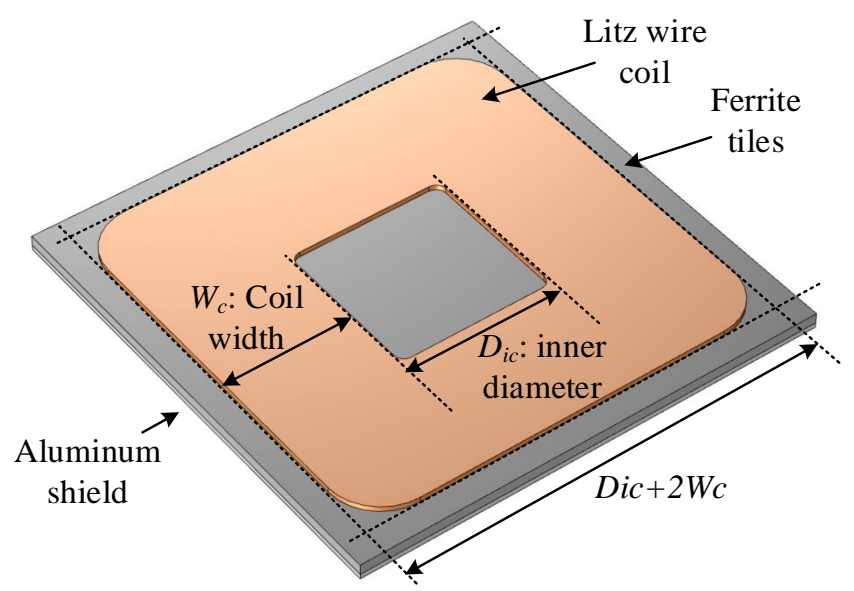

Figure 2.4: Structure of a square magnetic coupler for inductive EV charging systems.

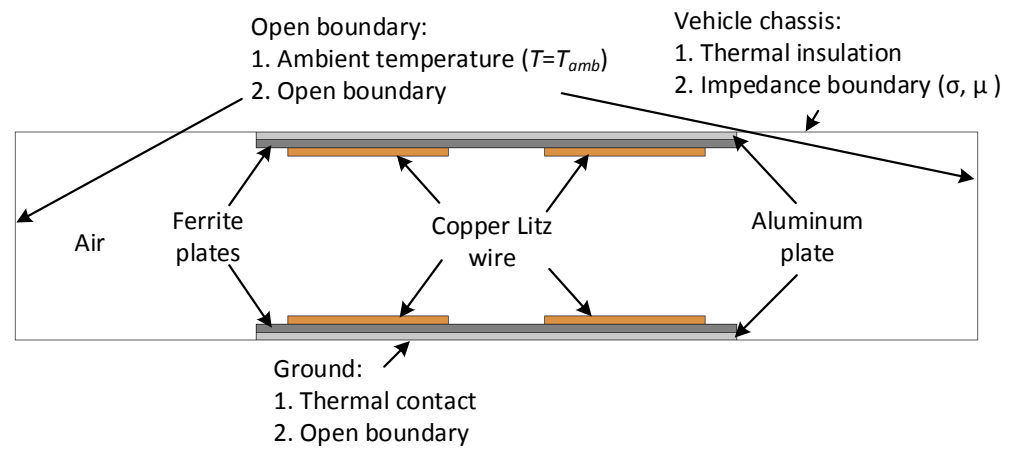

Figure 2.5: Boundary conditions of the hybrid physics model solved using FEA.

\subsubsection{Parametric Physics Model Using 3D Finite Element Model}

In order to be able to evaluate different design objectives and constraints in an inductive charging system with any design parameters, a parametric hybrid physics model that incorporates both electromagnetic and thermal models is employed. 3D Finite Element Analysis (FEA) is employed for numerically solving the physics model. In this study, COMSOL Multiphysics software is employed for performing the 3D FEAs. A physics model takes design parameters as an input and provides mutual- 


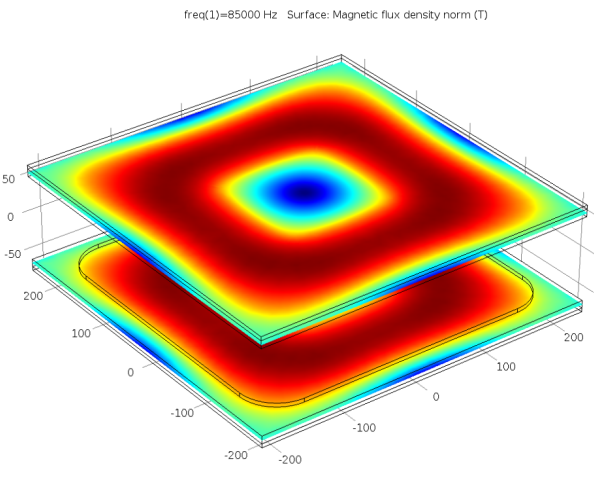

(a)

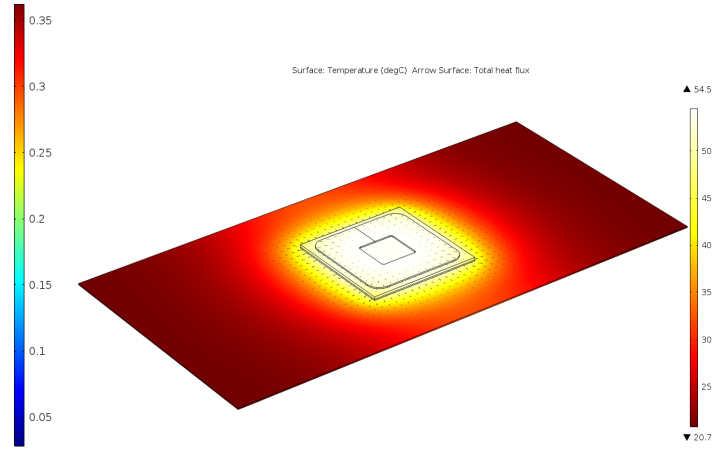

(b)

Figure 2.6: Sample finite element analysis results of the hybrid physics model: (a) Magnetic flux density distribution, (b) Temperature distribution.

inductance, magnetic field distribution, maximum temperature. The physics model can be directly coupled to the optimization algorithm. Fig. 2.4 shows a 3D FEA model of a square magnetic structure. In Fig. 2.5, the details of the applied boundary conditions for the electromagnetic and thermal models are presented. Further, in Fig. 2.6(a) and (b), sample plots of magnetic flux density distribution and temperature distribution on the magnetic structures are presented. In the following section, the derivation of different parameters using the physics model is presented.

\subsubsection{Equivalent Resistances of Coil}

Due to the high operating frequency of inductive charging systems, the coils are usually composed of Litz wire to minimize the losses by mitigating the skin and proximity effects. Litz wire is composed of many thin wire strands which are twisted and woven and are individually insulated. The skin and proximity effects in a Litz wire are divided into bundle level and strand level. Since the bundle-level effects are minimized by manufacturing techniques, in this study only strand-level effects are considered. The equivalent resistance that incorporates both skin and proximity 
effects can be expressed as [177],

$$
r_{\text {eq }}=r_{\text {skin }}+\left(r_{\text {proxi }}+r_{\text {proxe }}\right)
$$

where $r_{\text {skin }}$ is the strand-level skin effect resistance calculated as,

$$
r_{s k i n}=r_{d c} \frac{\gamma_{s}}{2} \frac{\operatorname{ber}\left(\gamma_{s}\right) \operatorname{bei}^{\prime}\left(\gamma_{s}\right)-\operatorname{bei}\left(\gamma_{s}\right) \operatorname{ber}^{\prime}\left(\gamma_{s}\right)}{\operatorname{ber}^{\prime 2}\left(\gamma_{s}\right)+b e i^{\prime 2}\left(\gamma_{s}\right)}
$$

$r_{d c}=\frac{4 \rho}{n_{s} \pi d_{s}^{2}} N l_{T}$ is the dc resistance of a strand, $\gamma_{s}=\sqrt{\omega \mu \sigma} r_{s}$, and $\operatorname{bei}(), \operatorname{ber}(), \operatorname{bei}^{\prime}(), \operatorname{ber}^{\prime}()$ are Kelvin functions. Also, $r_{\text {proxi }}$ and $r_{\text {proxe }}$ are the strand-level proximity effect resistances due to the internal magnetic field of the wire and external magnetic field, respectively and are calculated as [177],

$$
\begin{gathered}
r_{\text {proxi }}=-r_{d c} \frac{\beta \gamma_{s}}{2} \frac{\operatorname{ber}_{2}\left(\gamma_{s}\right) \operatorname{ber}^{\prime}\left(\gamma_{s}\right)+b e i_{2}\left(\gamma_{s}\right) b e i^{\prime}\left(\gamma_{s}\right)}{\operatorname{ber}^{2}\left(\gamma_{s}\right)+b e i^{2}\left(\gamma_{s}\right)} \\
r_{\text {proxe }}=n_{s} \frac{\operatorname{ber}_{2}\left(\gamma_{s}\right) \operatorname{ber}^{\prime}\left(\gamma_{s}\right)+b e i_{2}\left(\gamma_{s}\right) b e i^{\prime}\left(\gamma_{s}\right)}{\operatorname{ber}^{2}\left(\gamma_{s}\right)+b e i^{2}\left(\gamma_{s}\right)} \sum_{i=1}^{N} C_{i} \frac{H_{i, e x t}}{I_{r m s}^{2}}
\end{gathered}
$$

where $\beta$ is the packing factor, ber2(), bei2() are second order Kelvin functions, $H_{i, e x t}$ is the external magnetic field at the $i$ 'th turn which can be calculated using the electromagnetic model.

\subsubsection{Equivalent Resistance of Core Losses}

The ferrite core losses is calculated by integrating the Steinmetz equation over the volume of the core as,

$$
P_{\text {core }}=\int_{V_{f}} k f^{\alpha} \hat{B}^{\beta} d v
$$

where $P$ is the core loss per unit volume, $\hat{B}$ is the peak flux density, $f$ is the frequency of sinusoidal excitation, and $k, \alpha, \beta$ are Steinmetz constants which are determined using curve fitting techniques. Using (2.26), the equivalent series resistance which represents the core losses $r_{\text {core }}$ is calculated as,

$$
r_{\text {core }}=\frac{P_{\text {core }}}{I_{r m s}^{2}}=\frac{k f^{\alpha}}{I_{r m s}^{2}} \int_{V_{f}} \hat{B}^{\beta} d v
$$


The flux density distribution $\hat{B}$ is calculated using the electromagnetic physics model and thereby, $r_{\text {core }}$ can be directly calculated using $(2.27)$ by performing the integration over the volume of the core.

\subsubsection{Equivalent Resistance of Stray Losses}

The stray magnetic fields generated by the magnetic structures of inductive charging systems can penetrate into nearby conductive or magnetic object and induce losses. Due to high frequency and high magnitude characteristics of the stray magnetic fields, such losses can be significant. In inductive electric vehicle charging systems, such stray losses are induced in the shielding plates and the vehicle chassis. The losses on conductive objects are calculated using the surface impedance technique as $[178]$,

$$
P_{\text {plate }}=\int_{S} \sqrt{\frac{\omega \mu}{2 \sigma}} H_{r m s}^{2} d s
$$

where $H_{r m s}$ is the RMS magnetic field at the surface of the object which is calculated using the FEA. The equivalent series resistance that represents the stray losses $\left(r_{\text {stray }}\right)$ can be calculated as,

$$
r_{\text {stray }}=\sqrt{\frac{\omega \mu}{2 \sigma}} \frac{\int_{S} H_{r m s}^{2} d s}{I_{r m s}^{2}}
$$

\subsubsection{Equivalent Resistance of Compensation Capacitors}

The Equivalent Series Resistance (ESR) of the compensation capacitors can be calculated based on the loss tangent as,

$$
r_{c a p}=\frac{\tan \delta(\omega)}{\omega C}
$$

where $C$ is the capacitance of the capacitor and $\tan \delta(\omega)$ is the frequency dependent loss factor, which is provided by the capacitor manufacturer. 


\subsubsection{Thermal Performance Analysis}

In order to ensure that magnetic structures have a proper thermal performance, the thermal analysis is essential. In this study, the electromagnetic model is coupled to a thermal model in order to calculate the temperature distribution in the magnetic structures. The hot-spot temperature $\left(\theta_{h s}\right)$ is then determined and is used as a constraint in the optimization algorithm.

\subsubsection{Multi-Objective Optimization of Magnetic Structure Design}

\subsubsection{Multi-objective Optimization Formulation}

The design of magnetic structures in an inductive power system deals with multiple objectives including Power Transfer Efficiency ( $\eta$, Horizontal Misalignment Tolerance $(\Delta)$, Power Density $(\alpha)$, and Cost Efficiency $(\beta)$. The inductive charging system should be optimized with respect to different design parameters, such as coil inner width, coil width, number of turns, etc., to balance these four conflicting design objectives. In addition, multiple constraints need to be considered for the optimization problem. These constraints contain structural size limits, magnetic coupling constraint, and hot-spot temperature limit, and stray field limit to meet the desired design specifications and comply with standards.

Let $\boldsymbol{p}=\left(p_{1}, \ldots, p_{n}\right)$ denote $n$ design parameters of the inductive charging system, the magnetic structure design therefore can be defined as a multi-objective 
optimization problem (MOP) mathematically described as follows:

$$
\begin{aligned}
& \max \boldsymbol{F}(\boldsymbol{p})=\{\eta(\boldsymbol{p}), \Delta(\boldsymbol{p}), \alpha(\boldsymbol{p}), \beta(\boldsymbol{p})\} \\
& \text { s.t. } \quad S_{p}(\boldsymbol{p}) \leq S_{\max }, \\
& B_{s}(\boldsymbol{p}) \leq B_{\max }, \\
& \theta_{h s}(\boldsymbol{p}) \leq \theta_{\max }, \\
& M(\boldsymbol{p})=M_{0},
\end{aligned}
$$

where $\boldsymbol{F}(\cdot)$ is the set of four real-valued objective functions $\{\eta(\cdot), \Delta(\cdot), \alpha(\cdot), \beta(\cdot)\}$ defined as:

\subsubsection{Power Transfer Efficiency $(\eta)$}

The power transfer efficiency is one of the key performance metrics in inductive charging systems. This objective can be determined using (2.17). This equation is coupled to the electromagnetic physics model through $M, r_{1}$ and $r_{2}$ circuit parameters. It should be noted that losses in the coils, magnetic cores, and stray losses in the primary and secondary are all incorporated in $r_{1}$ and $r_{2}$, respectively.

\subsubsection{Horizontal Misalignment Tolerance $(\Delta)$}

Inductive EV charging systems should be able to tolerate slight horizontal misalignments by maintaining an acceptable power transfer efficiency. Higher tolerance to misalignments provides more convenience and flexibility for inductive EV charging stations. The misalignment tolerance for any magnetic structure can be calculated using the FEA model and power transfer efficiency calculations at different misalignments. The misalignment tolerance $(\Delta)$ is defined as $\Delta=\operatorname{Max}\left\{D \mid \eta(D) \geq \eta_{\min }\right\}$, where $D$ is the horizontal displacement and $\eta_{\min }$ is the minimum acceptable power transfer efficiency which should be $80 \%$ according to SAE J2954. 


\subsubsection{Power Density $(\alpha)$}

The power density is a measure of compactness of the magnetic structure which is determined as,

$$
\alpha=\frac{P}{V_{s}}
$$

where $\alpha$ is the power density usually expressed in $k W / d m^{3}$, and $V_{s}$ is the volume of the magnetic structure.

\subsubsection{Specific Cost $(\beta)$}

The specific material cost of the magnetic structure (in $W / \$$ ) can be determined by calculating the length of Litz wire, ferrite core volume, and shielding plate area as,

$$
\beta=\frac{P}{L_{w} C_{w}+V_{f} C_{f}+S_{p} C_{p}}
$$

where $L_{w}$ is the wire length, $V_{f}$ is the core volume, $S_{p}$ is the shielding plate area and $C_{w}, C_{f}, C_{p}$ are the per unit specific costs for the Litz wire, ferrite core and aluminum plate, respectively.

Let $\Omega=\left\{\boldsymbol{p} \in \mathbb{R}^{n} \mid S_{p} \leq S_{\text {max }}, B_{s}(\boldsymbol{p}) \leq B_{\max }, \theta_{h s}(\boldsymbol{p}) \leq \theta_{\max }, M(\boldsymbol{p})=M_{0}\right\}$ be the space of system design parameters. Geometric Constraint: $S_{p}(\boldsymbol{p}) \leq S_{\text {max }}$ represents the outer diameter of the pads and should be less than a maximum allowable value; Stray Field Constraint: $B_{s}(\boldsymbol{p}) \leq B_{\max }$ provides the maximum allowable magnetic field in a certain region as defined by the SAE J2954 standard; Temperature Limit: $\theta_{h s}(\boldsymbol{p}) \leq \theta_{\max }$ gives the maximum allowable operating temperature to ensure the thermal performance of the system; and Mutual-Inductance Constraint: $M(\boldsymbol{p})=M_{0}$ represents the desired mutual inductance constraint according to (2.21). 


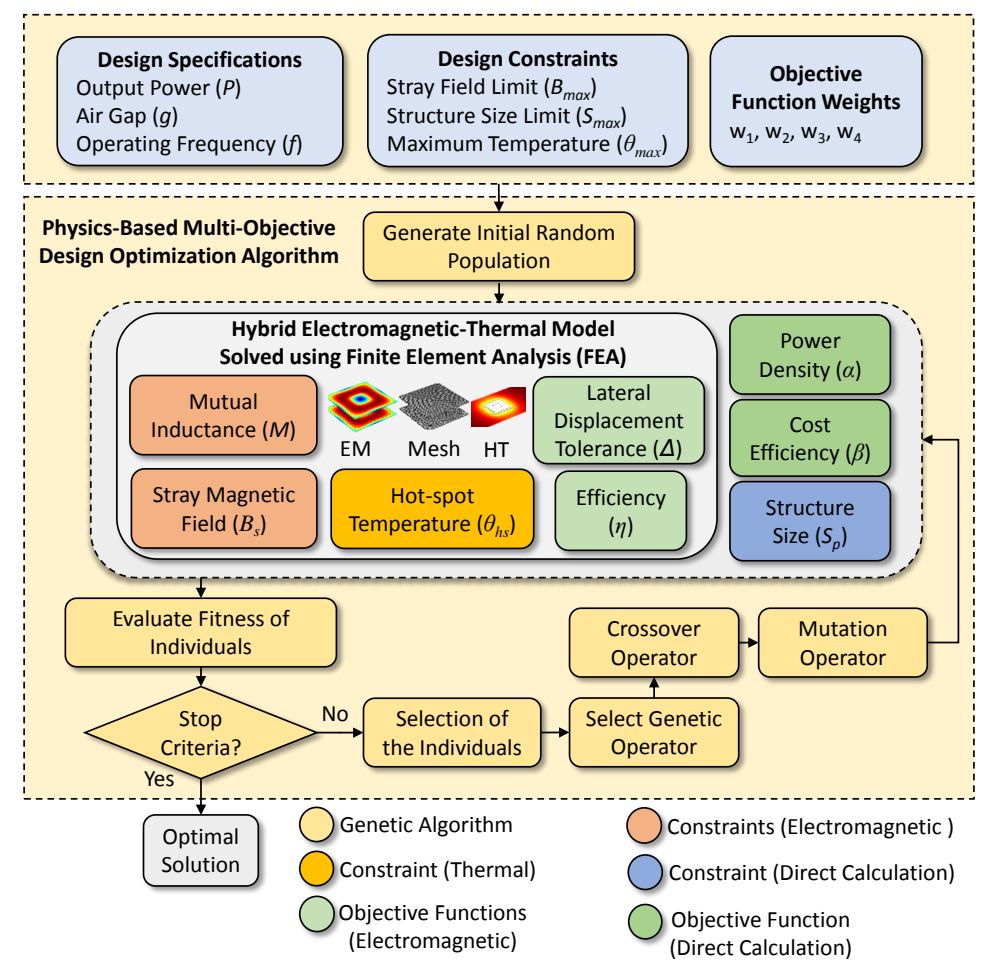

Figure 2.7: The flowchart of the proposed physics-based multi-objective design optimization algorithm.

\subsubsection{Genetic Algorithms for Multi-objective Optimization}

Given the design parameter space $\Omega$, the main objective of Problem (2.31) is to calculate a Pareto-optimal solution $\boldsymbol{p}^{*} \in \Omega$, where there is no $\boldsymbol{p} \in \Omega$ satisfying that, $\eta\left(\boldsymbol{p}^{*}\right) \leq \eta(\boldsymbol{p}), \Delta\left(\boldsymbol{p}^{*}\right) \leq \Delta(\boldsymbol{p}), \alpha\left(\boldsymbol{p}^{*}\right) \leq \alpha(\boldsymbol{p}), \beta\left(\boldsymbol{p}^{*}\right) \leq \beta(\boldsymbol{p})$, with at least one strict inequality fulfilled. However, due to the nonlinearity of the objective functions and constraints in Problem (2.31), the Pareto-optimal solution $\boldsymbol{p}^{*} \in \Omega$ cannot be computed efficiently [179]. Therefore, the scalarization method is implemented to solve Problem (2.31) by combining its four objective functions into one single-objective scalar function. For details, the scalarization method maximizes a 
positively weighted convex sum of the objectives, that is,

$$
\begin{array}{ll}
\max & w_{1} \eta(\boldsymbol{p})+w_{2} \Delta(\boldsymbol{p})+w_{3} \alpha(\boldsymbol{p})+w_{4} \beta(\boldsymbol{p}) \\
\text { s.t. } & \boldsymbol{p} \in \Omega,
\end{array}
$$

where $\boldsymbol{w}=\left[w_{1}, w_{2}, w_{3}, w_{4}\right]^{T}$ denotes for the weight vector, which is determined based on $\sum_{i=1}^{4} w_{i}=1$ and $w_{i}>0, i=1, \ldots, 4$. According to the theorems in [180], assume that the parameter space $\Omega$ is convex and the four objective functions $\{\eta(\boldsymbol{p}), \Delta(\boldsymbol{p}), \alpha(\boldsymbol{p}), \beta(\boldsymbol{p})\}$ are convex on $\Omega$, it can be proved that the optimal solution of the single objective Problem (2.34) is an efficient solution for the original MOP Problem (2.31). Corresponding to each weight vector $\boldsymbol{w}$, one of the Pareto-optimal solutions can be derived for the magnetic structure design problem.

Give a weight vector $\boldsymbol{w}$, the Genetic Algorithm (GA) is introduced to compute the optimal solution $\boldsymbol{p}^{*}$ of Problem (2.34) to determine the optimal design parameters of the magnetic structure for inductive charging systems. The proposed GA contains four main steps including: 1) First Population Initialization, 2) Selection of the Individuals, 3) Crossover, and 4) Mutation Process [181]. Firstly, the design parameters are represented in the chromosome type, typically as strings of 0 and 1. The GA begins with randomly generating a first population in the chromosome type. Then, the GA algorithm produces a series of new populations generated by selection, crossover and mutation processes. The optimal solution of Problem (2.34) is derived until the new population satisfies the algorithm stopping criteria. The flowchart of the proposed physics-based multi-objective design optimization algorithm is presented in Fig. 2.7. 
Table 2.1: The cost coefficients for different materials.

\begin{tabular}{lll}
\hline Coefficient & Description & Value \\
\hline$C_{w}$ & Litz wire cost per unit length $(\$ / \mathrm{m})$ & 2.04 \\
$C_{f}$ & Ferrite material cost per unit volume $\left(\$ / \mathrm{dm}^{3}\right)$ & 180 \\
$C_{p}$ & Aluminum plate cost in unit area $\left(\$ / \mathrm{m}^{2}\right)$ & 333.68 \\
\hline
\end{tabular}

Table 2.2: Design specifications of the target case study system.

Description
Output power $(P)$
Frequency $\left(f_{s}\right)$
Secondary Battery Voltage $\left(V_{B}\right)$
Maximum Structure Footprint $\left(S_{p}\right)$
Maximum Stray Field $\left(B_{\max }\right)$
Maximum Hot-Spot Temperature

(a)

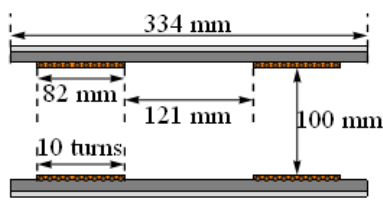

(c)

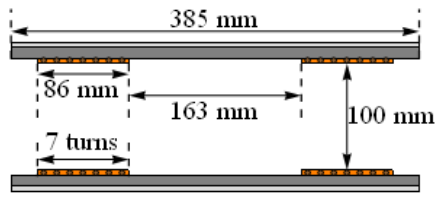

(e)

Value

$100 \mathrm{~mm}$ (Class Z1)

$3.7 \mathrm{~kW}$ (Class WPT1)

$85 \mathrm{kHz}$

$160 \mathrm{~V}$

$25 d m^{2}$

$6.25 \mu \mathrm{T}$

$80^{\circ} \mathrm{C}$

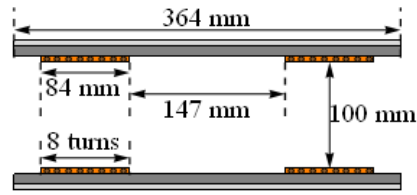

(b)

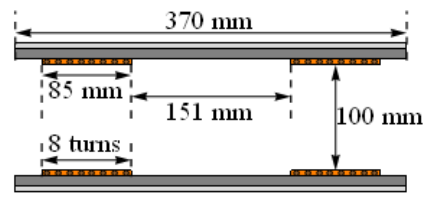

(d)

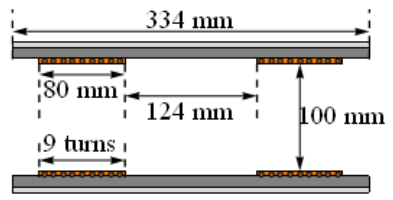

(f)

Figure 2.8: Optimized magnetic structures for different design cases of Table 2.3.

\subsubsection{Case Study Analysis}

A $3.7 \mathrm{~kW}$ inductive EV charging system for $100 \mathrm{~mm}$ air gap (WPT1/Z1 as defined in SAE J2954) with square magnetic couplers is investigated as the case study. The magnetic structures are assumed to have an area no more than $25 \mathrm{dm}^{2}$. Also, the stray field of the inductive system should be less than $6.25 \mu \mathrm{T}$ to meet human 


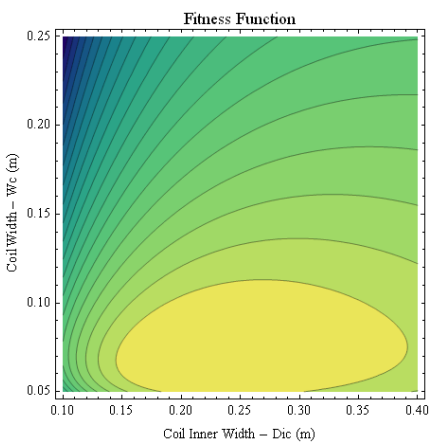

(a)

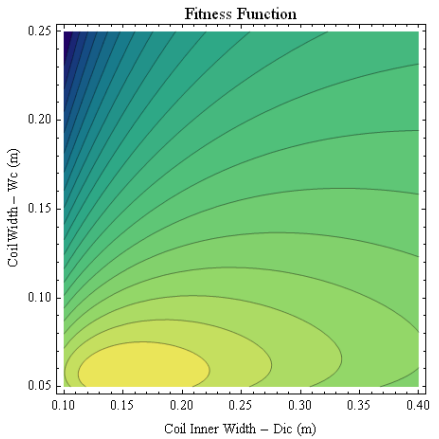

(d)

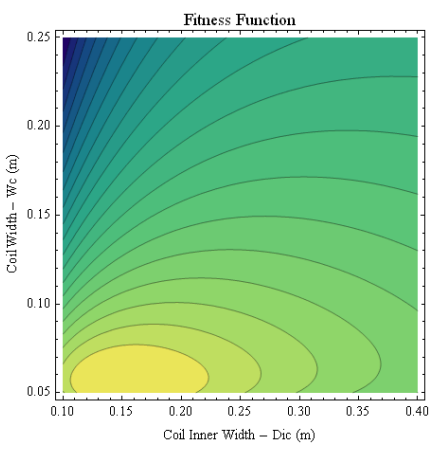

(b)

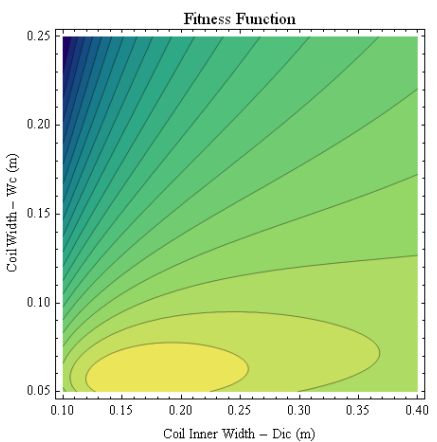

(e)

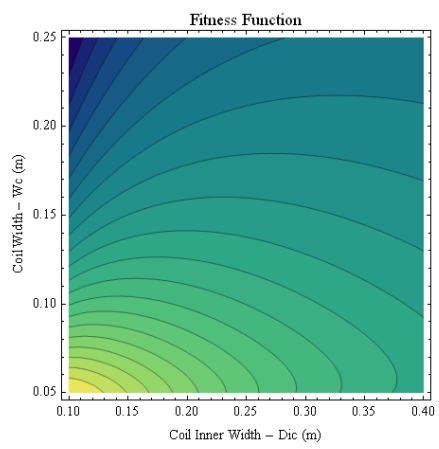

(c)

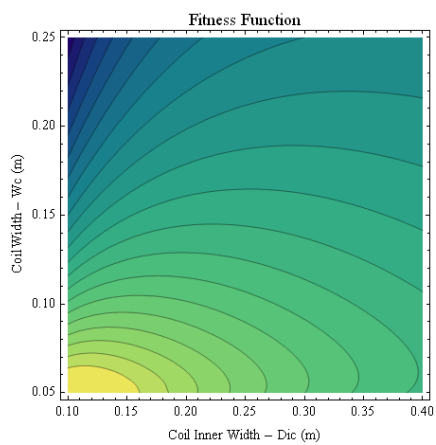

(f)

Figure 2.9: Contour plots of fitness function for different design cases of Table 2.3.

Table 2.3: Optimization results for different design scenarios.

\begin{tabular}{|c|c|c|c|c|c|c|c|c|c|c|c|c|}
\hline \multirow{2}{*}{ No. } & \multicolumn{4}{|c|}{ Objective Function Weights } & \multicolumn{3}{|c|}{ Optimized Design Parameters } & \multicolumn{5}{|c|}{ Objective Functions and Constraints } \\
\hline & $w_{1}$ & $w_{2}$ & $w_{3}$ & $w_{4}$ & $D_{i c}(m m)$ & $W_{c}(m m)$ & $N$ & $\eta(\%)$ & $\Delta(m m)$ & $\alpha\left(k W / d m^{3}\right)$ & $\beta(W / \$)$ & $B_{s}(\mu T)$ \\
\hline 1 & 0.7 & 0.1 & 0.1 & 0.1 & 223 & 103 & 5 & 96.81 & 164 & 0.81 & 46.68 & 3.45 \\
\hline 2 & 0.4 & 0.1 & 0.4 & 0.1 & 147 & 84 & 8 & 95.82 & 116 & 1.39 & 80.11 & 4.98 \\
\hline 3 & 0.3 & 0.1 & 0.5 & 0.1 & 121 & 82 & 10 & 95.07 & 102 & 1.65 & 94.94 & 5.94 \\
\hline 4 & 0.4 & 0.4 & 0.1 & 0.1 & 151 & 85 & 8 & 95.92 & 117 & 1.35 & 77.75 & 5.02 \\
\hline 5 & 0.3 & 0.5 & 0.1 & 0.1 & 163 & 86 & 7 & 96.15 & 125 & 1.25 & 71.86 & 4.47 \\
\hline 6 & 0.3 & 0.1 & 0.1 & 0.5 & 124 & 80 & 9 & 95.15 & 102 & 1.65 & 94.94 & 5.37 \\
\hline
\end{tabular}

exposure regulations in compliance with ICNIRP 2010 standard [91]. Also, the ferrite tiles are selected as MnZn N87 material. The cost function is calculated using the cost coefficients presented in Table 2.1. The detailed design requirements and constraints are presented in Table 2.2.

The parameterized square magnetic structure is presented in Fig. 2.4. As it is shown, the magnetic structure is identified by inner width of the coil $\left(D_{i c}\right)$, width of the coil $\left(W_{c}\right)$, and number of turns $\left(N_{t}\right)$. The proposed multi-objective optimization 
algorithm is programmed in MATLAB. Also, COMSOL Multiphysics is employed for performing parametric 3D FEAs on electromagnetic and thermal physics models. The multi-objective optimization is carried out for different design priorities by applying corresponding objective function weights to achieve optimal solutions. A computer with a Core i7-4770 processor and 16 GB of memory is employed to perform the optimizations and the results are presented in Table 2.3. Each 3D FEA takes 75 seconds on average. The average time required for each optimization scenario is about 9 hours. However, by utilizing the multi-core processing capabilities, multiple FEAs can be performed in parallel to significantly reduce the optimization time. In Fig. 2.9, the corresponding contour of the fitness function for each design case is presented. Also, the optimized design structure for each design case is presented in Fig. 2.8.

\subsubsection{Design for Efficiency}

Case No. 1 of Table 2.3 shows the optimization for results maximum power transfer efficiency. This is achieved by assigning the highest priority to the efficiency and minimum priority to the rest of objectives. The corresponding optimized magnetic structure is presented in Fig. 2.8(a). As it can be seen the optimized structure requires to have a large cross-sectional area with only 5 turns. Although using this design $96.81 \%$ efficiency can be achieved, the power density of this design is as low as $0.81 \mathrm{~kW} / \mathrm{dm}^{3}$.

\subsubsection{Design for Efficiency and Power Density}

In case No. 2, the power transfer efficiency and power density are considered with the same level of priority. Compared to case No. 1, although the efficiency is reduced 
by about $1 \%$, the power density is improved by $72 \% .\left(1.39 \mathrm{~kW} / \mathrm{dm}^{3}\right)$. The optimized structure for this case study is shown in Fig. 2.8(b).

\subsubsection{Design for Power Density}

The power density can be further improved by increasing its priority over efficiency as presented for Case No. 3. Compared to Case No. 2, the power density is improved by about $19 \%$ while the efficiency is decreased by $0.75 \%$. The dimensions of the optimized structure is presented in Fig. 2.8 (c) and the corresponding contour plot of the fitness function is presented in Fig. 2.9(c).

\subsubsection{Design for Efficiency and Displacement Tolerance}

The cases No. 4 and 5 present optimal solutions the priority of displacement tolerance. Specifically, the optimized structure in case No. 5 can achieve $125 \mathrm{~mm}$ displacement tolerance with $96.15 \%$ efficiency and $1.25 \mathrm{~kW} / \mathrm{dm}^{3}$ power density. The corresponding structures and objective function contours are presented in Fig. 2.8 (d),(e) and in Fig. 2.9(d),(e).

\subsubsection{Design for Cost Efficiency}

Case No. 6, presents an optimized design for both power transfer efficiency and cost efficiency. The derived design parameters and objective function values are similar to case No. $3\left(1.65 \mathrm{~kW} / \mathrm{dm}^{3}\right)$ with slight improvement in efficiency. This is due to the fact that the power density and cost efficiency objectives follow the same trend. The corresponding optimized structure and the objective function contour are presented in Fig. 2.8 (f) and in Fig. 2.9(f). 
Table 2.4: Specifications of the experimental setup.

\begin{tabular}{|c|c|}
\hline Component & Description \\
\hline Ferrite Plates & Laird MP2106-0M0, $53 \mathrm{~mm} \times 53 \mathrm{~mm} \times 2.5 \mathrm{~mm}, \mathrm{~N} 87 \mathrm{MnZn}$ \\
\hline Litz Wire & Gauge 8 , round Litz wire, $5 \times 5 \times 42 / 38$ structure \\
\hline AC Capacitors & KEMET PHE450, film capacitor $22 n F, 3 \mathrm{kV}$ \\
\hline Power Switches & IXYS IXXN110N65C4H1, 650V 210A IGBT \\
\hline Resistive Loads & 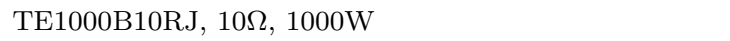 \\
\hline
\end{tabular}

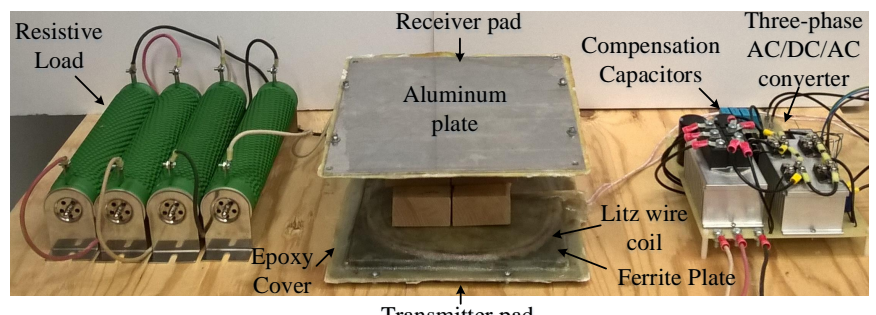

Transmitter pad

Figure 2.10: The developed experimental setup with optimized square magnetic structures designed for high power density.

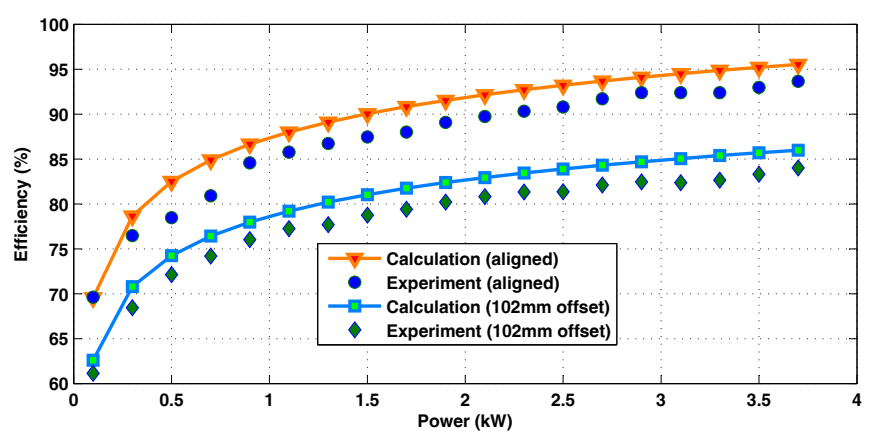

Figure 2.11: Power transfer efficiency of the case study system as function of receiver power obtained using both calculations and experimental measurements with and without horizontal offset.

\subsubsection{Experimental Analysis}

Based on the optimization results presented in Section 2.1.4 for maximum power density, a $3.7 \mathrm{~kW}$ prototype is built. The magnetic structure is built according to the design parameters of case No. 3 of Table 2.3 which are derived for highest power density. The experimental setup is shown in Fig. 2.10 and the specification are presented in Table 2.4. The setup is composed of optimized square magnetic 
structures, a three-phase power supply, a three-phase $\mathrm{AC} / \mathrm{DC} / \mathrm{AC}$ converter, and secondary loads.

Fig. 2.11 shows the plots of the power transfer efficiency $(\eta)$ of the case study system as functions of power $(\mathrm{kW})$ which are obtained using both calculations and experimental measurements with and without horizontal offset. According to Fig. 2.11, the experiments show that the prototype system can achieve $93.65 \%$ efficiency at $3.7 \mathrm{~kW}$ which is good agreement with $95.53 \%$ theoretical value. Also, it can be seen that with $102 \mathrm{~mm}$ horizontal offset, the efficiency of the system at $3.7 \mathrm{~kW}$ is reduced to $83.43 \%$ which conforms to the expected horizontal displacement tolerance. 


\section{CHAPTER 3}

\section{DESIGN AND DEVELOPMENT OF SELF-TUNING CONTROLLERS FOR INDUCTIVE POWER TRANSFER SYSTEMS}

In this chapter, self-tuning controllers for IPT systems with resonant current control are introduced. The proposed controllers are designed based on an amplitude modulation technique for resonant converters to regulate the transferred power in an IPT system. Using the proposed controllers, the switching operations of the converters are synchronized to the resonance current of the IPT system which in turn eliminates the need for manual frequency tuning and enables soft-switching operations (zero-current switching). Soft-switching operations increase the efficiency and reliability, and reduce the switching stress and electromagnetic interference (EMI) of the converters. Based on the control design, a simplified digital control circuit is proposed which can be used as an alternative for high-cost DSP/FPGA solutions. The design methodology, theoretical analysis of the proposed converter, along with simulation and experimental results on a case study IPT system are presented in detail. The results show that the proposed controllers can effectively regulate the transferred power with self-tuning capability and soft-switching operations. The work presented in this chapter is published in [162,172-174].

\subsection{Sliding Mode Controller for H-Bridge Converters in In- ductive Power Transfer Systems}

In this section, soft-switching self-tuning H-bridge converters which are controlled based on the an amplitude modulation technique based on a sliding mode control are proposed for IPT systems. The proposed controller benefits from the self-tuning capability. This enables the synchronization of the switching operations with the 
resonant current and guarantees soft-switching operations. A simplified digital design for the proposed controller is presented which can be used as an alternative for DSP/FPGA based solutions. It can operate at much higher frequencies which is suitable for IPT applications. The proposed converter is analyzed theoretically and simulated in MATLAB/Simulink, and finally, it is implemented experimentally and the results are presented.

\subsubsection{Self-Tuning Controller Design}

In Fig. 3.1, an H-bridge converter topology connected to a DC source input and an equivalent RLC circuit of an IPT system at the output is shown. The DC source is usually a full-bridge rectifier. The equivalent circuit of an IPT system is composed a series capacitance $C$, primary inductance $L$, and an equivalent resistance $R_{e q}$ which represents the reflected load from the secondary circuit to the primary. The self-tuning controller for an H-bridge converter can be designed based on the energyinjection and free-oscillation technique for resonant circuits. A conceptual plot of the resonant current and output voltage of a converter which are controlled using energy-injection control method is shown in Fig. 3.2. As it is shown, each half-cycle can be either an energy-injection mode or a free-oscillation mode. The transitions between different modes only occur at resonant current zero-crossing points, which ensures the soft-switching operation of the converter. In energy-injection modes, energy is injected into the LC tank from the DC voltage source, thus increasing the resonant current. On the other hand, in free-oscillation modes, the LC tank continues its oscillation without energy injection from the source, thus decreasing the resonant current. Therefore, the resonant current can be regulated around a reference current by constantly switching between the two operation modes. 


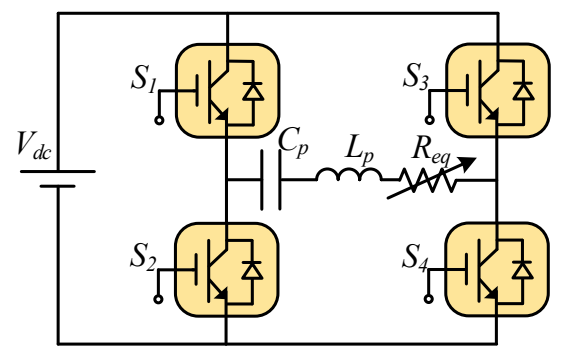

Figure 3.1: An H-bridge converter connected to an equivalent RLC circuit representing an IPT system.

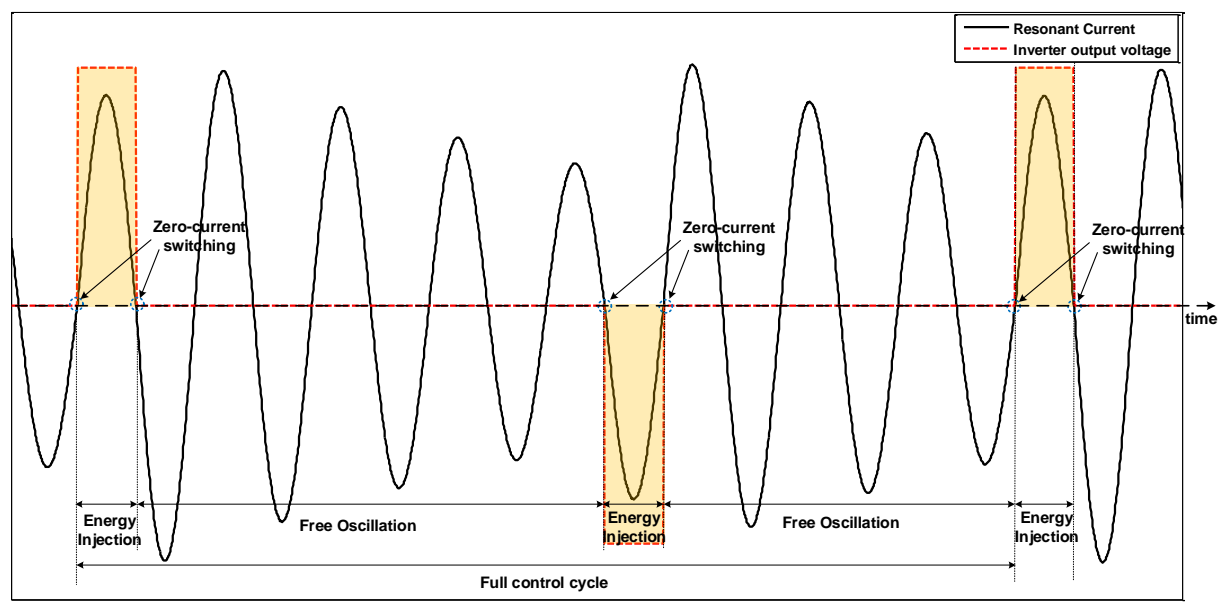

Figure 3.2: Resonant current and output voltage of the H-bridge converter which is controlled using the proposed SMC based on energy injection and free oscillation technique.

\subsubsection{Sliding Mode Controller Design}

The controller can be designed based on sliding mode control (SMC) framework which is based on energy-injection and free-oscillation technique to perform amplitude modulation on the resonant current. The sliding surface is defined based on the peak resonant current as,

$$
\sigma[k]=i_{\text {ref }}-\left|i_{p}[k]\right|
$$


where $\sigma[k]$ is the discrete sliding surface and $i_{r e f}$ is the reference current and $i_{p}[k]$ is the discretized peak resonant current can be rewritten as follows [133]:

$$
i_{p}[k]=\left(u[k]-v_{c 1}[k]\right) \frac{\tau e^{-\arctan (\tau \omega) / \tau \omega}}{L \sqrt{1+(\tau \omega)^{2}}}
$$

where $u[k]$ is the input voltage of the IPT system, $v_{c 1}[k]$ is the voltage of the compensation capacitor, $\tau$ is the resonant damping time constant, and $\omega$ is the natural resonance frequency. The reaching law for the SMC can be formulated as follows [182]:

$$
(\sigma[k+1]-\sigma[k]) \sigma[k]<0
$$

Using (3.1) and (3.3) the following can be derived:

$$
\left(\left|i_{p}[k+1]\right|-\left|i_{p}[k]\right|\right) \sigma[k]>0
$$

Based on (3.4) the feedback control law $u[k]$ is picked so that the discrepancy between consecutive resonant current peaks and $\sigma[k]$ have same signs. In other words, whenever $\sigma[k]>0$ energy injection to the LC tank should be performed to increase the peak resonant current and whenever $\sigma[k]<0$ the LC tank should continue its free-oscillation. This is depicted in Fig. 3.2. In an H-bridge converter which is shown Fig. 3.1, the output voltage which is applied to the LC tank can be either $V_{d c},-V_{d c}$ or 0 . As a result, based on (3.4) the control law for a full-bridge converter can be derived as,

$$
u[k+1]= \begin{cases}V_{d c} & \sigma[k]>0, i_{p}[k]<0 \\ -V_{d c} & \sigma[k]>0, i_{p}[k]>0 \\ 0 & \sigma[k]<0\end{cases}
$$

Based on (3.5), an H-bridge converter will have four operation modes which are presented in Table 3.1. These operation modes are determined according to the 

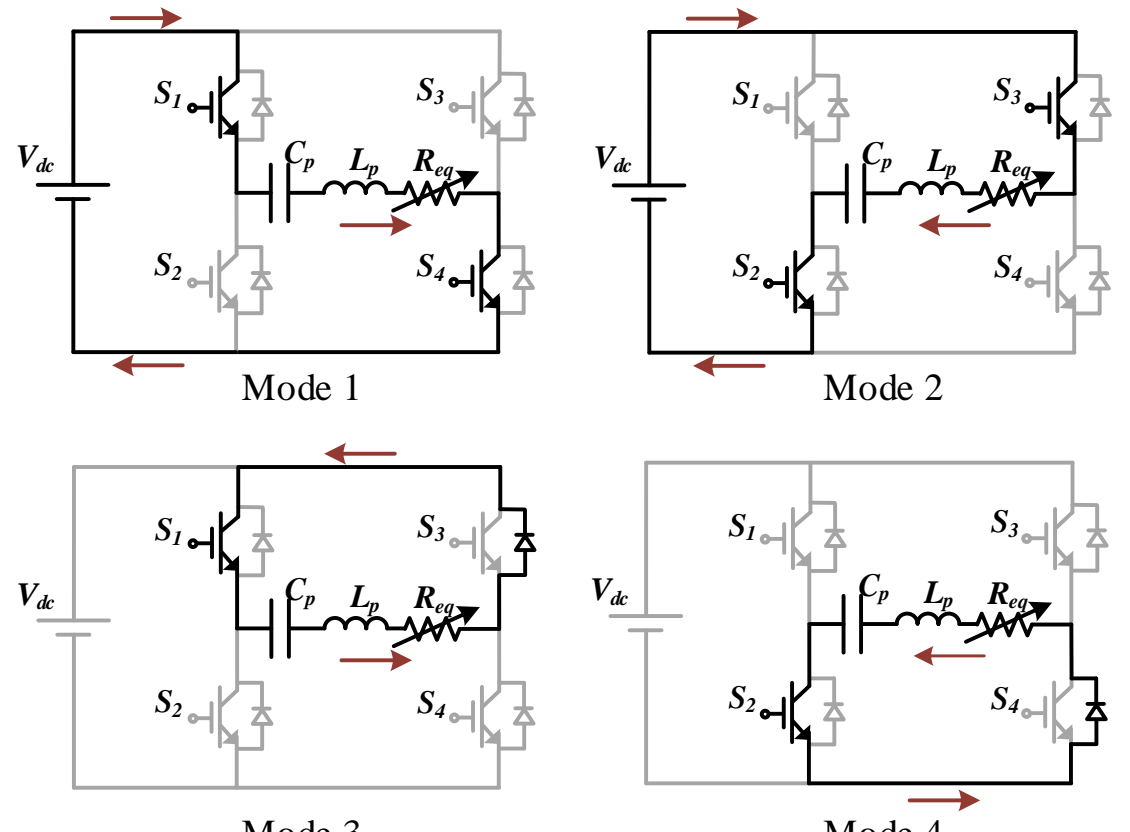

Mode 3

Mode 4

Figure 3.3: Different control modes in an H-bridge converter: energy injection modes (1 and 2), free-oscillation modes (3 and 4).

Table 3.1: Four operation modes and corresponding switching states in an H-bridge converter.

\begin{tabular}{cc|cc|cccc}
\hline Mode & Type & $\operatorname{sign}\left(i_{r}\right)$ & $\operatorname{sign}(\sigma[k])$ & $S_{1}$ & $S_{2}$ & $S_{3}$ & $S_{4}$ \\
\hline \hline 1 & energy injection & 1 & 1 & 1 & 0 & 0 & 1 \\
2 & energy injection & 0 & 1 & 0 & 1 & 1 & 0 \\
3 & free oscillation & 1 & 0 & 1 & 0 & 0 & 0 \\
4 & free oscillation & 0 & 0 & 0 & 1 & 0 & 0 \\
\hline
\end{tabular}

sign of $\sigma$ and peak resonant current $i_{p}$ in each half-cycle. In Fig. 3.3, the resonant current path in four different operation modes are presented. According to Table 3.1 and Fig. 3.3, the switching states of the H-bridge converter would be as follows:

$$
\begin{gathered}
S_{1}=\operatorname{sign}\left(i_{r}\right) \quad S_{2}=\overline{\operatorname{sign}\left(i_{r}\right)} \\
S_{3}=\overline{\operatorname{sign}\left(i_{r}\right)} \cdot \operatorname{sign}(\sigma[k]) \quad S_{4}=\operatorname{sign}\left(i_{r}\right) \cdot \operatorname{sign}(\sigma[k])
\end{gathered}
$$




\subsubsection{Simplified Design for the Sliding Mode Controller}

Based on the control laws and corresponding switching signals derived for the $\mathrm{H}$ bridge converter in Section 3.1.1.1, a simplified digital controller can be designed which is shown in Fig. 3.4. The controller takes the resonant current of the IPT system and the reference current as the inputs and generates four switching signals for the H-bridge converter. The performance of the converter can be described in four operation modes, which are presented in Table 3.1 and Fig. 3.3. The operation modes 1 and 2 are energy injection modes in which energy is injected into the IPT system, and the operation modes 3 and 4 are free oscillation modes in which the IPT system continues its resonant oscillation. The transitions to different modes of operation only occur at resonant current zero-crossing points. The controller takes a feedback from the resonant current of the IPT system as the input and generates the switching signals for the four switches of the H-bridge converter. It is composed of two differential voltage comparators, a peak detector, two D-type flip-flops, two AND gates, and a NOT gate.

The first differential comparator is used to detect resonant current zero-crossing points, as well as its direction. The peak detector is used to detect the peak of the resonant current in each half-cycle. The D-type flip-flops are used to save the state of the peak comparator for the next half-cycle $(\operatorname{sign}(\sigma[k]))$. These two flip-flops are

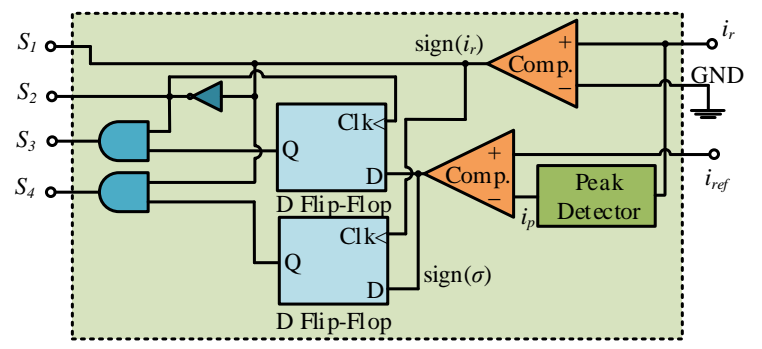

Figure 3.4: The proposed simplified controller for H-bridge resonant converters ( $i_{r}$ is the measured resonant current signal, $i_{r e f}$ is the reference current). 


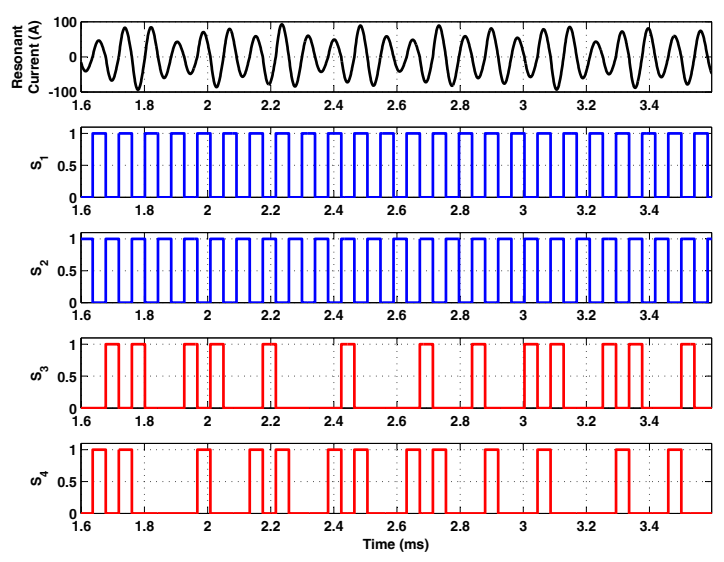

(a)
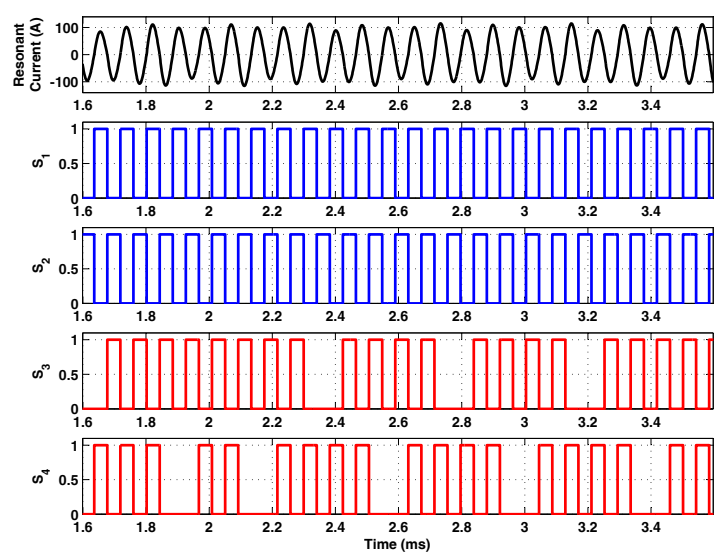

(b)

Figure 3.5: Simulation results on the case study IPT system using an H-bridge converter topology: (a) $i_{\text {ref }}=40 \mathrm{~A}, P_{\text {out }}=6.6 \mathrm{~kW}$, (b) $i_{\text {ref }}=60 \mathrm{~A}, P_{\text {out }}=14 \mathrm{~kW}$.

used to consider both positive and negative peaks of the resonant current. Finally, AND and NOT gates are used to generate the appropriate switching signals for $S_{1}$, $S_{2}, S_{3}$ and $S_{4}$ according to Table 3.1 .

\subsubsection{Simulation Analysis}

The proposed self-tuning soft-switching control circuit for H-bridge converter topology which is presented in Fig. 3.4, is simulated using MATLAB/Simulink. The simulation model is comprised of a three-phase mains, transmitter and receiver pads with their corresponding compensation circuits, an AC/DC/AC H-bridge converter which is controlled by the proposed controller and it is connected to the transmitter coil, and a battery charger for an electric vehicle at the secondary. The self-inductances of the primary and secondary are each $172 \mu H$, where each has a $120 n F$ compensation capacitor. As a result, the operating resonance frequency of the LC tank will be $35 \mathrm{kHz}$. The three-phase power supply has a line-to-line voltage of $208 \mathrm{~V}$ with $60 \mathrm{~Hz}$ power frequency. 


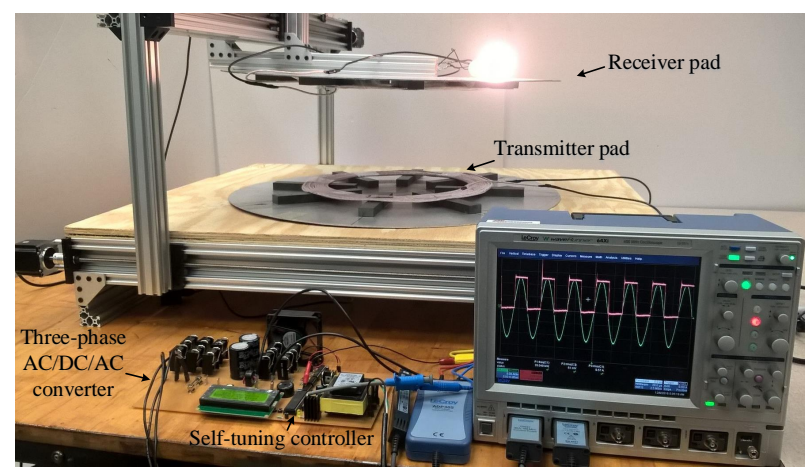

Figure 3.6: The case study IPT system with circular pads as transmitter and receiver, an $\mathrm{AC} / \mathrm{DC} / \mathrm{AC}$ converter controlled by the proposed control circuit.

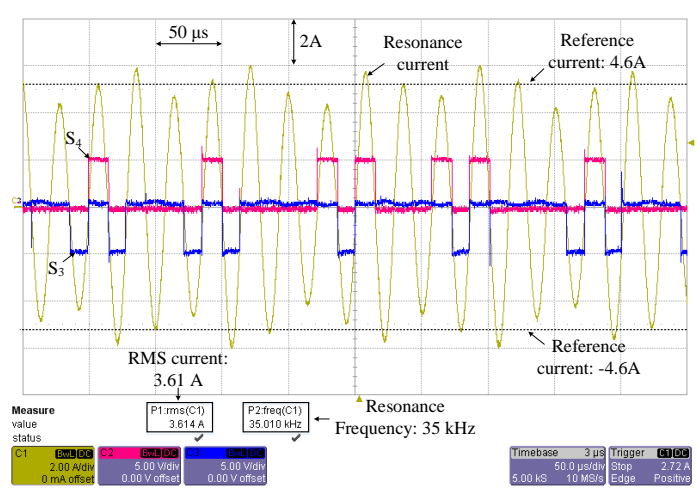

(a)

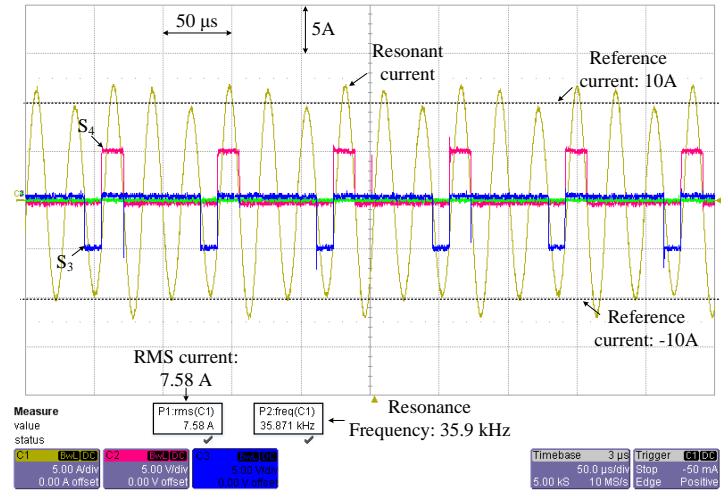

(b)

Figure 3.7: Resonant current and energy injection switching signals of the H-bridge converter in the case study IPT system: (a) $V_{L L}=10 \mathrm{~V}, i_{\text {ref }}=3.6 \mathrm{~A}, P_{\text {out }}=35 \mathrm{~W}$, (b) $V_{L L}=20 \mathrm{~V}, i_{\text {ref }}=10 \mathrm{~A}, P_{\text {out }}=155 \mathrm{~W}$.

The reference current of the controller $\left(i_{\text {ref }}\right)$ is set to $60 \mathrm{~A}$ and $100 \mathrm{~A}$ and the simulations were carried out. In Figs. $3.5 \mathrm{a}$ and $3.5 \mathrm{~b}$, the resonant current, and the corresponding switching signals for both simulation cases are shown. As it can be seen, the switches 1 and 2 are constantly switching while switches 3 and 4 have a variable frequency switching signals. These switching signals are adjusted by the controller to regulate the energy injection to the IPT system in order to regulate the transferred power. 


\subsubsection{Experimental Analysis}

In order to verify the performance of the proposed self-tuning controller, a pro-totype H-bridge converter topology is built based on the control circuit presented in Fig. 3.4 and the experimental tests were carried out. The case study IPT system which is shown in Fig. 3.6 is comprised of two circular power pads as the transmitter and receiver structures, compensation capacitors, an AC/DC/AC H-bridge converter along with the proposed self-tuning controller. The self-inductance of the circular pads are each $172 \mu \mathrm{H}$, where each has a $120 n F$ compensation capacitor and thereby, the operating resonance frequency of the LC tank would be $35 \mathrm{kHz}$. A variable three-phase power supply is used as the AC mains. The experimental tests were carried out in two scenarios: (a) $V_{L L}=10 \mathrm{~V}$ and $i_{r e f}=3.6 \mathrm{~A}$, (b) $V_{L L}=20 \mathrm{~V}$ and $i_{r e f}=10 A$, where $V_{L L}$ is the line-to-line voltage of the three-phase input voltage. In Fig. 3.7, the resonant current and energy injection switching signals $\left(S_{3}\right.$ and $S_{4}$ ) of the H-bridge converter are shown. These results show that the implemented controller is capable of regulating the resonance current around the reference current at different input voltage levels with soft-switching operations.

\subsection{Self-Tuning Variable Frequency Controller for Inductive Electric Vehicle Charging with Multiple Power Levels}

In this section, a self-tuning controller for contactless electric vehicle (EV) charging systems based on inductive power transfer (IPT) with multiple power levels is introduced. The multiple charging levels (consisting of 10 charging levels) are achieved by controlling the energy injection frequency of the transmitter coil of the inductive power transfer (IPT) system. The charging levels include the standard 
wireless charging levels for light-duty EVs (Levels 1, 2, 3 and 4) as defined by SAE TIR J2954 [90]. The proposed controller is capable of self-tuning the switching operations to the natural resonance frequency of the IPT system and benefits from soft-switching operations (zero-current switching), which ensures the maximum performance of the IPT system. The proposed controller has such a simple design which can be implemented based on a simplified control circuit. The simulation of the proposed controller for an inductive charging system at different charging levels is carried out in MATLAB/Simulink. Also, the proposed controller with an $\mathrm{AC} / \mathrm{DC} / \mathrm{AC}$ converter is implemented experimentally on an IPT charging system to verify the effectiveness of the controller at different charging levels. The experimental test results conform with the simulation results and verify that the proposed controller effectively enables self-tuning capability and soft-switching operations at different charging levels for IPT based contactless EV charging systems.

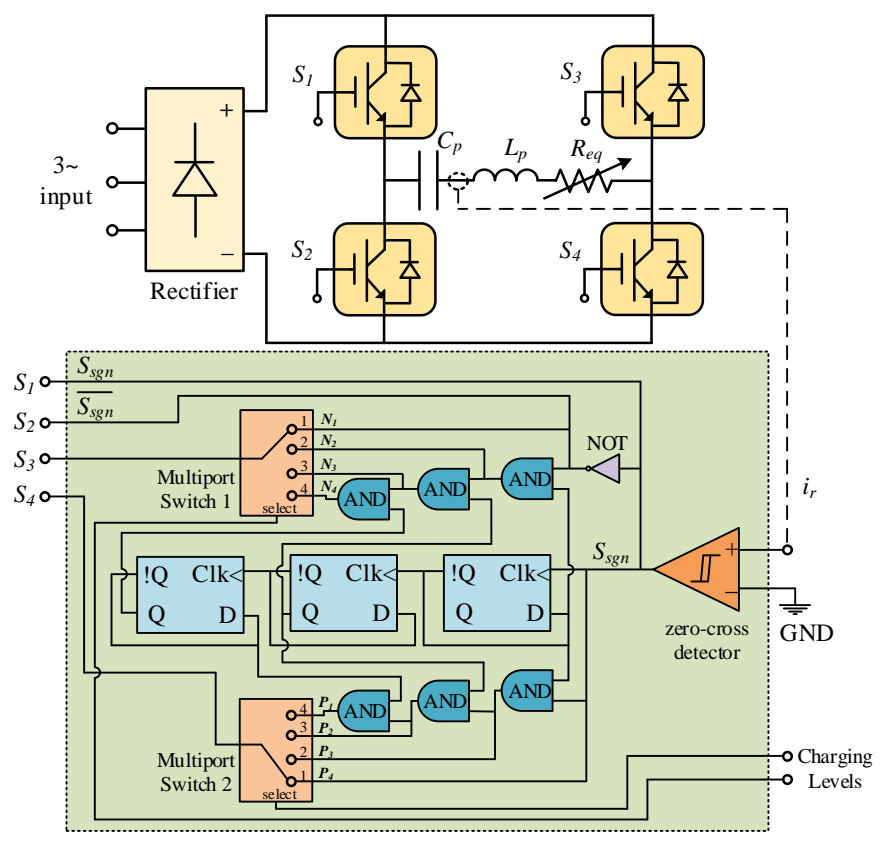

Figure 3.8: The proposed simplified control circuit designed for a two-stage fullbridge $\mathrm{AC} / \mathrm{DC} / \mathrm{HFAC}$ converter. 


\subsubsection{The Proposed Controller}

Using the energy injection method the amplitude of the resonant current in an IPT system can be controlled by varying the rate of the energy injection to the primary coil. On this basis, a self-tuning variable frequency energy injection control method for IPT systems is proposed. The proposed method employs a variable frequency energy injection to the IPT system to achieve multiple charging levels. Fig. 3.8 shows the proposed controller which is designed for a two-stage AC/DC/HFAC converter. The two-stage converter is composed of a three-phase rectifier and a single-phase full-bridge high-frequency inverter. The operation of the full-bridge inverter can be described in four modes based on the direction of the resonant current (positive or negative) and the type of the (energy injection or free-oscillation) operation mode, as it is presented in Fig. 3.3 and Table 3.2. Based on Table 3.2, four switching signals of the full-bridge inverter $S_{1}, S_{2}, S_{3}$, and $S_{4}$ can be expressed in terms of current direction $\left(S_{s g n}\right)$ and energy injection $\left(S_{i n j}\right)$ states as follows:

$$
S_{1}=S_{s g n}, \quad S_{2}=\overline{S_{s g n}}, \quad S_{3}=\overline{S_{s g n}} \cdot S_{i n j} \quad S_{4}=S_{s g n} \cdot S_{i n j}
$$

Based on (3.7), regardless of the type of operation mode (whether it is an energy injection or free-oscillation mode) $S_{1}$ is switched $\mathrm{ON}$ when the direction of current is positive, and $S_{2}$ is switched $\mathrm{ON}$ when the direction of current is negative. Furthermore, (3.7) shows that $S_{4}$ is switched $\mathrm{ON}$ in positive energy injection modes and $S_{3}$ is switched $\mathrm{ON}$ in negative energy injection modes.

Table 3.2: Four operation modes of a full-bridge inverter and corresponding switching states.

\begin{tabular}{|c|c|c|c|c|c|c|c|}
\hline Mode & Type & $\begin{array}{ll}\text { Current } & \text { direction } \\
\left(S_{s g n}\right) & \\
\end{array}$ & $\begin{array}{ll}\text { Energy } & \text { injection } \\
\left(S_{i n j}\right) & \\
\end{array}$ & $S_{1}$ & $S_{2}$ & $S_{3}$ & $S_{4}$ \\
\hline mode 1 & Energy injection & 1 & 1 & 1 & 0 & 0 & 1 \\
\hline mode 2 & Energy injection & 0 & 1 & 0 & 1 & 1 & 0 \\
\hline mode 3 & Free oscillation & 1 & 0 & 1 & 0 & 0 & 0 \\
\hline mode 4 & Free oscillation & 0 & 0 & 0 & 1 & 0 & 0 \\
\hline
\end{tabular}




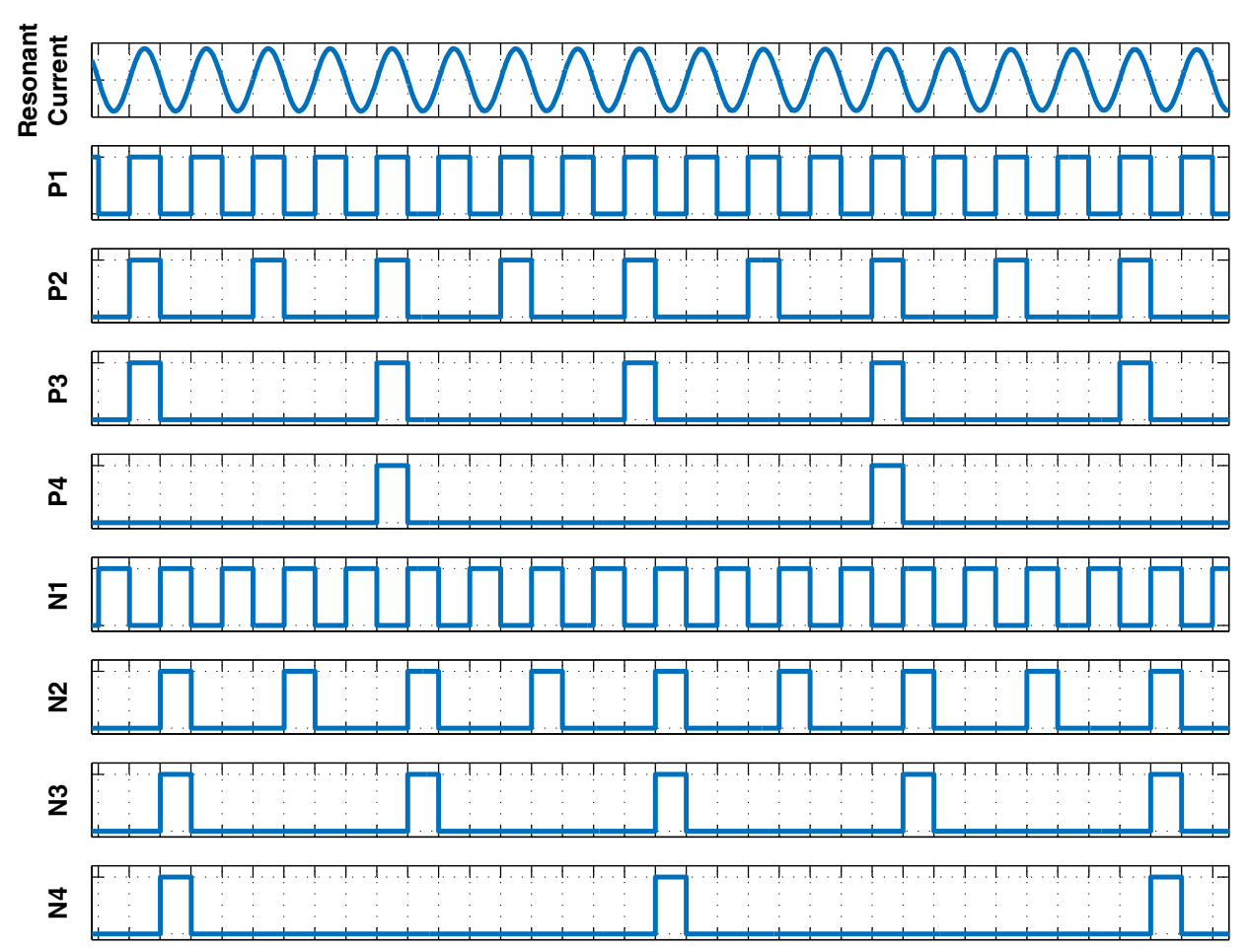

Figure 3.9: The energy injection switching signals with frequency of $f_{r}$ (resonance frequency), $f_{r} / 2, f_{r} / 4$ and $f_{r} / 8$ for positive $\left(P_{1}, P_{2}, P_{3}\right.$ and $\left.P_{4}\right)$ and negative $\left(N_{1}, N_{2}\right.$, $N_{3}$ and $N_{4}$ ) half-cycles which are generated generated by the proposed controller.

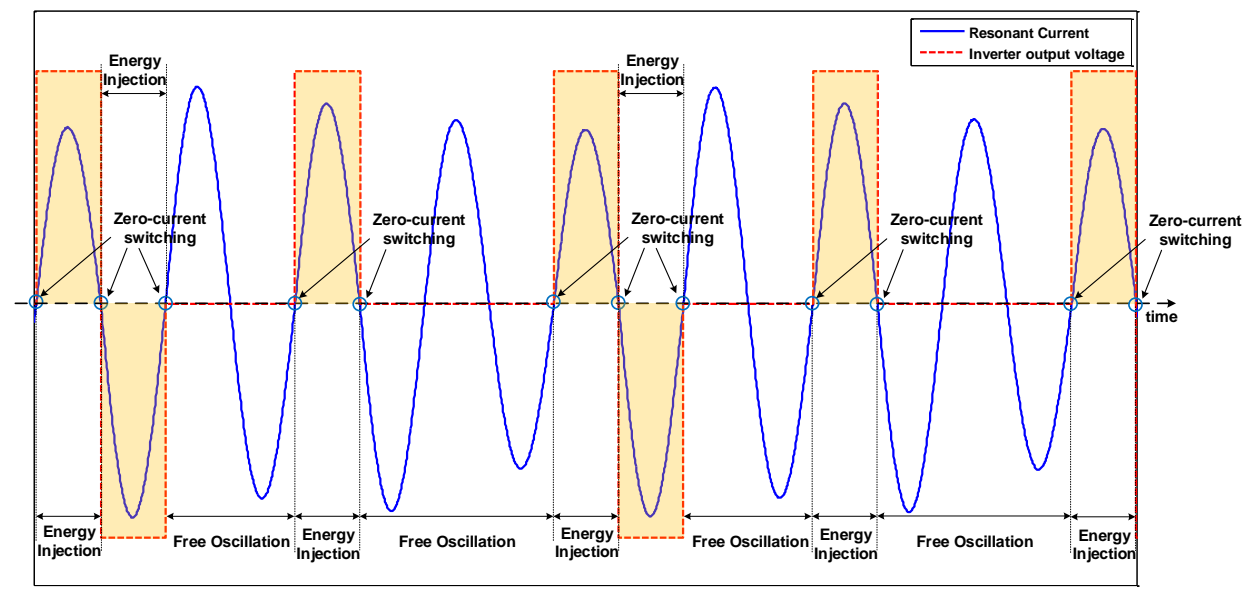

Figure 3.10: Typical waveforms of the resonant current and the converter output voltage applying the proposed control method for charging Level 2-4.

The presented controller is designed based on a simplified circuit and it is composed of D-type flip-flops, logic gates, a differential comparator, and multi-port 
switches. The sign of the resonant current $\left(S_{s g n}\right)$ which has the resonance frequency $f_{r}$, is determined by the zero-cross detector which is the differential comparator. Based on (3.7), $S_{1}$ is connected to $S_{s g n}$ and $S_{2}$ is connected to $\overline{S_{s g n}}$ as shown in Fig 3.8. The series flip-flops form three frequency dividers and by using the sign signal $S_{s g n}$ as the clock source, signals with $f_{r} / 2, f_{r} / 4$ and $f_{r} / 8$ frequencies are generated. By combining the sign signal $S_{\text {sgn }}$ with the signals generated by the frequency divider with the use of AND and NOT gates, four switching signals for positive energy injection half-cycles $\left(P_{1}, P_{2}, P_{3}, P_{4}\right)$ and four switching signals for negative energy injection half-cycles $\left(N_{1}, N_{2}, N_{3}, N_{4}\right)$ are generated. Based on (3.7), since $P_{1}, P_{2}, P_{3}$ and $P_{4}$ are energy injection signals in positive half-cycles, $S_{3}$ can be connected to any of them, enabling energy injection to the IPT system at different rates. Similarly, based on (3.7), since $N_{1}, N_{2}, N_{3}$ and $N_{4}$ energy injection signals in positive half-cycles, $S_{4}$ can be connected to any of them, enabling energy injection to the IPT system at different rates. Therefore, the multi-port switches are employed to connect $S_{3}$ and $S_{4}$ to the proper energy injection switching signals based on a user-defined charging level preset. In Fig. 3.9, typical energy injection switching signals for positive and negative half-cycles are presented. Using two multi-port switches in the proposed control circuit the frequency of energy injection in positive and negative half-cycles of the resonant current can be controlled separately. The energy injection frequency in positive and negative half-cycles can be $f_{r}$ (resonance frequency), $f_{r} / 2, f_{r} / 4$ and $f_{r} / 8$. Thereby, enabling energy injection to the IPT system at 10 distinct levels. These charging levels are presented in Table 3.3. Although, these $16(4 \times 4)$ charging levels can be reduced to 10 distinct charging levels as there are 6 charging levels are equivalent to the other 10 charging levels which are given in Table 3.3, e.g. Level 1-2 is equivalent to Level 2-1, and Level 1-4 is equivalent to Level 4-1, etc. 
Typical waveforms of the resonant current and output voltage of a full-bridge inverter with the use of energy injection control method is shown in Fig. 3.10. As it can be seen, each mode starts and ends at zero-crossing points, which leads to zerocurrent current switching (ZCS) of the inverter. Therefore, the converter will have higher efficiency compared to conventional converters. Also, the simple design of the controller not only simplifies its implementation but also enables higher operating frequencies, at which conventional digital controllers (DSP/FPGA) may not be able to achieve.

In charging levels with different energy injection frequencies for positive and negative half-cycles, the output voltage of the full-bridge inverter will have a DC component. Since the impedance of the series RLC circuit shown (in Fig. 2), for a DC input is infinite (the capacitor acts as an open circuit). In other words, the DC component of the voltage is eliminated. As a result, the output resonant current will only include harmonic (non-DC) components, without any DC component, which will result in a symmetrical resonant current.

In an IPT system if the converter operates at the resonance frequency a perfect matching will be obtained, as the impedance of the compensation capacitors cancels out the equivalent impedance of the transmitter and receiver coils, resulting in a purely resistive network; Thereby, there would be no phase difference between the resonant current and input voltage. As a result, the zero-current switching (ZCS) will ensure the zero-voltage switching (ZVS). Since using the proposed controller the switching operations are synchronized with the resonant current and therefore, the ZCS and ZVS are both achieved. However, if the resonance frequency of the primary and secondary are a little different due to imperfect resonance matching, the equivalent circuit would not be purely resistive. Since the proposed controller uses zero-current crossing points for generating the switching signals, in such a case only ZCS will be achieved. 


\subsubsection{Theoretical Analysis}

In this section theoretical calculation of different measures for the contactless charging system based on the proposed controller is presented. Analytical solutions for the resonant voltage, resonant current and the output power can be found as follows:

\subsubsection{Resonant Current and Resonant Voltage Calculation}

The differential equation of a series compensated IPT system can be expressed based on the resonant current $i$ as follows:

$$
\frac{d^{2} i}{d t^{2}}+\frac{R_{e q}}{L} \frac{d i}{d t}+\frac{1}{L C} i=0
$$

where $L$ is the self-inductance of the transmitter, and $C$ is the compensation capacitor of the transmitter, and $R_{e q}$ is the equivalent resistance of the load on the receiver side, reflected to the transmitter side. At each zero-crossing point of resonant current, the initial conditions of the circuit are as,

$$
i_{0}=0, \quad L \frac{d i}{d t}(0)=V_{t}-v_{c 0}
$$

where $i_{0}$ is the initial resonant current and $V_{t}$ is the output voltage of the inverter. The resonant current and the resonant voltage $\left(v_{c}\right)$ of the compensation capacitor

Table 3.3: Different charging levels which can be achieved using the proposed controller.

\begin{tabular}{c|cc}
\hline \hline & \multicolumn{2}{|c}{ Frequency of energy injection } \\
Charging Level & Positive half-cycles & Negative half-cycles \\
\hline Level 1-1 & $f_{r}$ & $f_{r}$ \\
Level 1-2 & $f_{r}$ & $f_{r} / 2$ \\
Level 1-4 & $f_{r}$ & $f_{r} / 4$ \\
Level 1-8 & $f_{r}$ & $f_{r} / 8$ \\
Level 2-2 & $f_{r} / 2$ & $f_{r} / 2$ \\
Level 2-4 & $f_{r} / 2$ & $f_{r} / 4$ \\
Level 2-8 & $f_{r} / 2$ & $f_{r} / 8$ \\
Level 4-4 & $f_{r} / 4$ & $f_{r} / 4$ \\
Level 4-8 & $f_{r} / 4$ & $f_{r} / 8$ \\
Level 8-8 & $f_{r} / 8$ & $f_{r} / 8$ \\
\hline
\end{tabular}


can be found by solving (3.8), based on the initial conditions given in (3.9) [133]:

$$
\begin{gathered}
i(t)=K e^{-t / \tau} \sin (\omega t) \\
v_{c}(t)=v_{c 0}+\frac{K \tau}{C\left(1+\tau^{2} \omega^{2}\right)}\left(\tau \omega-e^{-t / \tau}[\sin (\omega t)+\tau \omega \cos (\omega t)]\right)
\end{gathered}
$$

where $\tau=2 L / R$ is the damping time constant, $\omega=\sqrt{\omega_{0}^{2}-\alpha^{2}}$ is the natural damped frequency, $\omega_{0}=1 / \sqrt{L C}$ is the resonant frequency, $\alpha=R_{e q} / 2 L$ is the damping coefficient, and the coefficient $K$ is expressed as:

$$
K=\frac{V_{t}-v_{c 0}}{\omega L}
$$

In order to calculate the initial condition for the resonant voltage $v_{c 0}$ at each current zero-crossing in a steady-state condition, a full control cycle consisting of $2 n$ half-cycles of the resonant current which includes one energy injection half-cycle (Fig. 3.10) is considered. The resonant voltage at the end of the energy injection half-cycle $(t=\pi / \omega)$ can be calculated using (3.11) as follows:

$$
v_{c 1}=V_{t}+\beta\left(V_{t}-v_{c 0}\right)
$$

where $\beta$ is defined as:

$$
\beta=e^{\frac{-\pi}{\tau \omega}}
$$

At the end of free-oscillation half-cycles (half-cycles from 2 to $2 n$ ), the resonant voltage can be calculated based on (3.11) as follows:

$$
v_{c k}=v_{c 1} \beta^{k-1}(-1)^{k-1}
$$

Using (3.13), equation (3.15) can be rewritten as follows:

$$
v_{c k}=V_{t}(1+\beta) \beta^{k-1}(-1)^{k-1}+v_{c 0} \beta^{k}
$$

By assuming that the system has reached a steady-state condition, it can be concluded that the resonant voltage at the beginning of each control cycle $\left(v_{c 0}\right.$ at $\left.k=0\right)$ 
should be equal to the its value at the end of the control cycle $\left(v_{c k}\right.$ at $\left.k=2 n\right)$. Therefore, using (3.16) the following equations can be derived:

$$
\begin{gathered}
v_{c 0}=-V_{t}(1+\beta) \beta^{2 n-1}+v_{c 0} \beta^{2 n} \\
v_{c 0}=-\frac{(1+\beta)}{1-\beta^{2 n}} \beta^{2 n-1} V_{t}
\end{gathered}
$$

Equation (3.18) is the initial condition for the resonant voltage in the steadystate condition and can be used in $(3.12),(3.11)$ to calculate the resonant current and the resonant voltage at any time.

\subsubsection{Output Power Calculation}

The maximum output power of the converter is achieved when the controller is set to level 1-1. In this case, all of the half-cycles of the resonant current would be in energy injection mode. Using the same method for calculation of the initial condition for the resonant voltage in steady-state conditions which is presented in Section 3.2.2.1, the initial condition for the resonant voltage can be calculated as,

$$
v_{c 0}=-\frac{1+\beta}{1-\beta} V_{t}
$$

Using (3.12) and (3.19), the resonant current $i$ for any half-cycle can be written as follows:

$$
i=\frac{2 V_{t}}{\omega L(1-\beta)} e^{-t / \tau} \sin (\omega t)
$$

The output power can be calculated using (3.20) as follows:

$$
\begin{gathered}
P=\frac{\int_{0}^{\pi / \omega} V_{t} \frac{2 V_{t}}{\omega L(1-\beta)} e^{-t / \tau} \sin (\omega t) d t}{\pi / \omega} \\
P=\frac{2 V_{t}^{2} \tau^{2} \omega(1+\beta)}{\pi L(1-\beta)\left(1+\tau^{2} \omega^{2}\right)}
\end{gathered}
$$

Using (3.22), the output power can be calculated based on the input voltage and the circuit parameters. 
Table 3.4: The simulation results at charging levels 1-1, 1-2, 2-2 and 4-8.

\begin{tabular}{c|lc|c}
\hline Charging Level & $\begin{array}{l}\text { RMS Resonant } \\
\text { Current (A) }\end{array}$ & THD of Current (\%) & Output Power (kW) \\
\hline \hline Level 1-1 & 105.2 & 0.85 & 41 \\
Level 1-2 & 78.4 & 2.18 & 22.1 \\
Level 2-2 & 67 & 3.43 & 12.3 \\
Level 4-8 & 55.1 & 5.39 & 3.6 \\
\hline
\end{tabular}

\subsubsection{Simulation Results}

An inductive EV charging system based on the proposed self-tuning controller which is shown in Fig. 3.8, is simulated in MATLAB/Simulink. The simulation model is presented in Fig. 3.11 and it is composed of a three-phase power supply, a fullbridge three-phase rectifier, a full-bridge single-phase inverter which is switched by the proposed controller, transmitter and receiver coils with their compensation capacitors, and a battery charger for an EV at the receiver. The self-inductances of the transmitter and receiver each are assumed to be $172 \mu H$, with compensation capacitors of $0.12 \mu F$. Therefore, the resonance frequency of the IPT system is 35 $\mathrm{kHz}$. The three-phase power supply is assumed to have a line-to-line voltage of 208 $\mathrm{V}$ and $60 \mathrm{~Hz}$ power frequency. The EV battery is modeled as a $360 \mathrm{~V}$ battery with

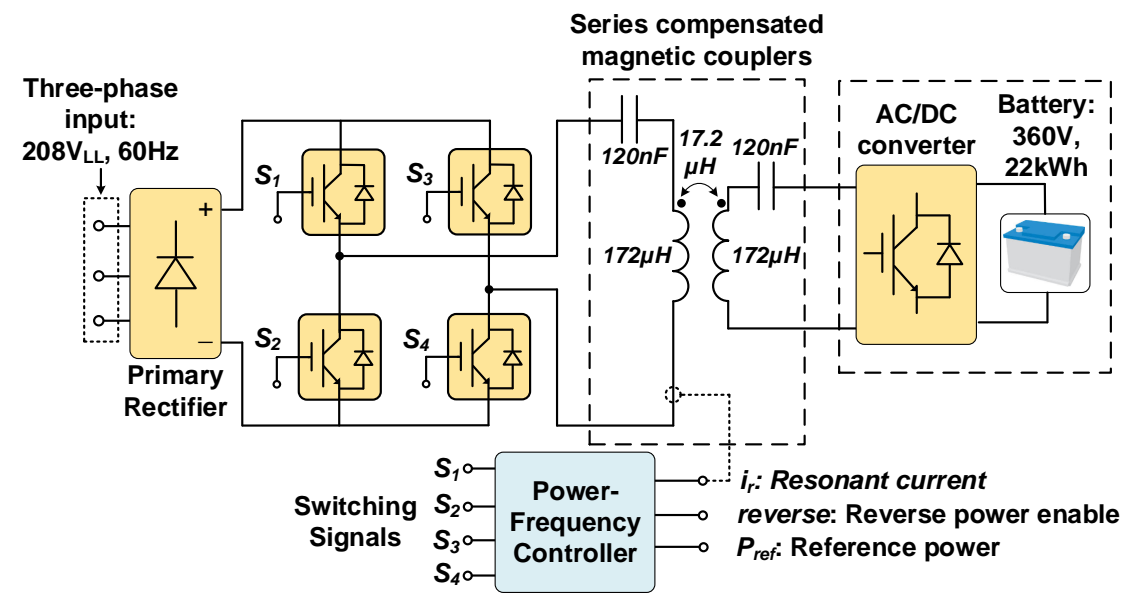

Figure 3.11: Inductive electric vehicle battery charging model with the proposed controller simulated in MATLAB/Simulink. 


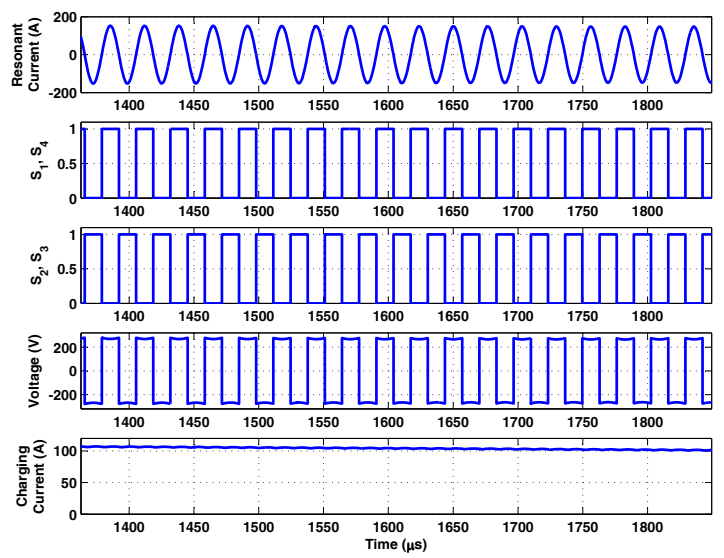

(a)

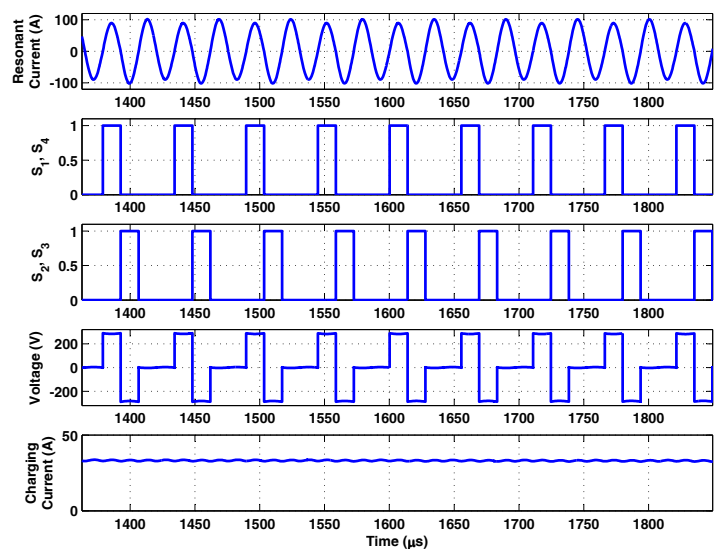

(c)
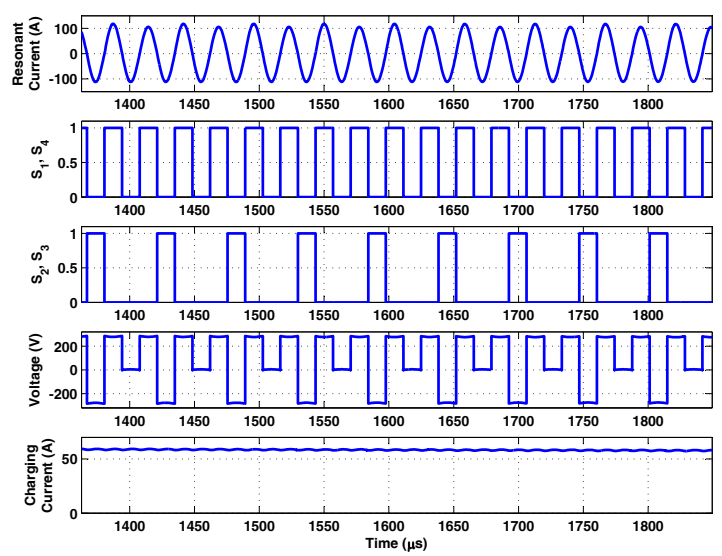

(b)
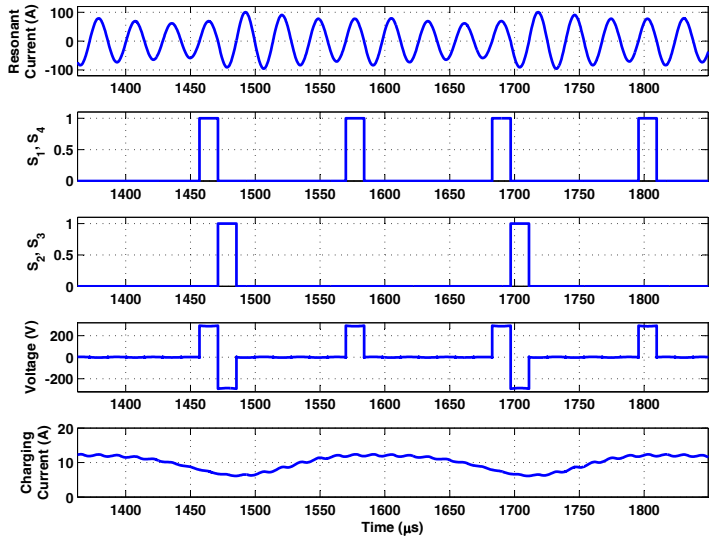

(d)

Figure 3.12: The resonant current, inverter output voltage, switching signals and battery charging current in different charging levels: (a) Level 1-1 charging with 41 kW output power, (b) Level 1-2 charging with $22.1 \mathrm{~kW}$ output power, (c) Level 2-2 charging with $12.3 \mathrm{~kW}$ output power, (d) Level $4-8$ charging with $3.6 \mathrm{~kW}$ output power.

$22 \mathrm{kWh}$ capacity. The secondary converter is modeled as a conventional two-stage $(\mathrm{AC} / \mathrm{DC} / \mathrm{DC})$ battery charger that can operate at constant current $(\mathrm{CC})$ mode at a desired charging power level.

The simulations are carried out in four charging levels (levels 1-1, 1-2, 2-2 and 4-8 according to Table 3.3) and the results are presented in Table 3.4. Also, the resonant current, inverter output voltage, battery charging current and correspond- 


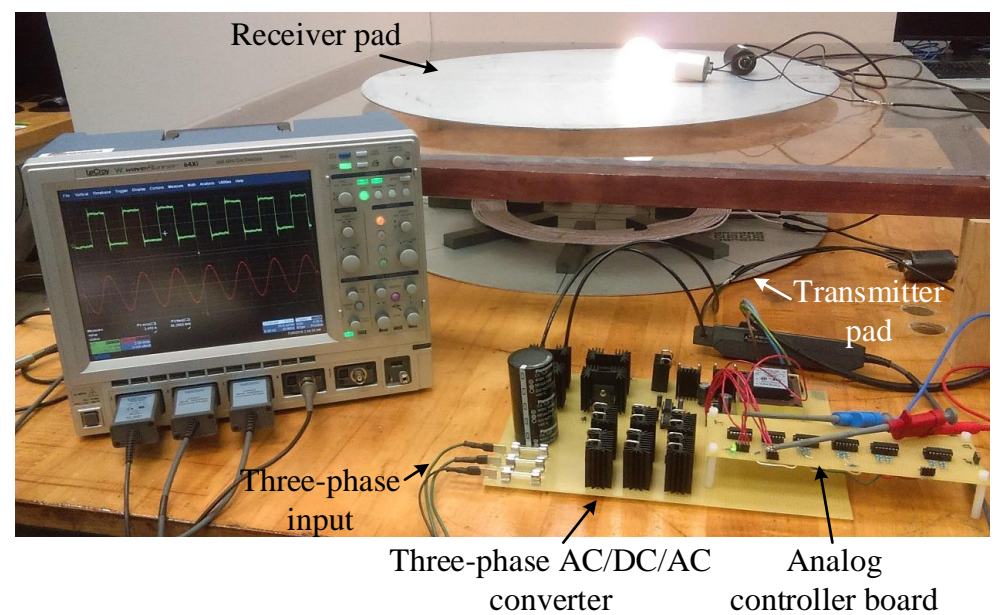

Figure 3.13: The case study IPT setup consisting of two circular transmitter and receiver power pads, an $\mathrm{AC} / \mathrm{DC} / \mathrm{AC}$ converter as the primary converter and the proposed controller.

ing switching signals are shown in Fig. 3.12. As it is shown, using the proposed controller, different charging levels can be achieved with low harmonic distortions (THD) in the resonant current. It is important to note that the charging levels 1-2 and 4-8 respectively correspond to the charging levels 4 and 1 , as defined by SAE TIR J2954 standard [90].

\subsubsection{Experimental Analysis}

The proposed controller for IPT systems is implemented experimentally and tested on an IPT system. In Fig. 3.13, the IPT test-bed which is comprised of two circular transmitter and receiver power pads, compensation capacitors, a threephase $\mathrm{AC} / \mathrm{DC} / \mathrm{AC}$ converter as the primary converter connected to the proposed controller, and a variable load at the secondary. The self-inductance of the power pads is $172 \mu \mathrm{H}$, and each pad has a $0.12 \mu \mathrm{F}$ compensation capacitor. As a result, the resonance frequency of the IPT system would be $35 \mathrm{kHz}$. The three-phase input 


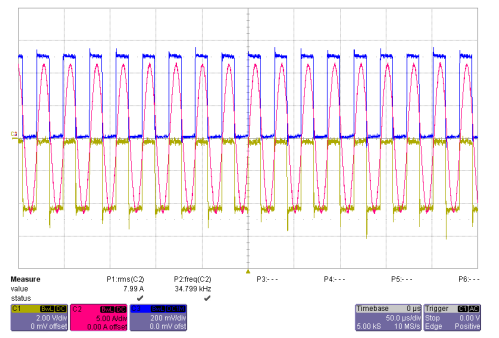

(a) Level 1-1

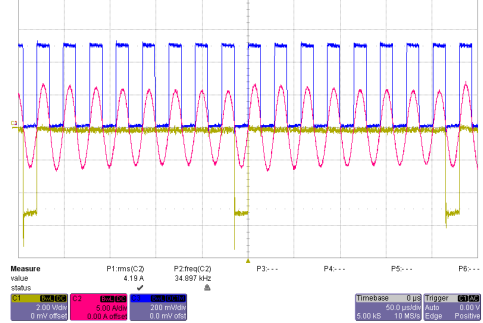

(d) Level 1-8

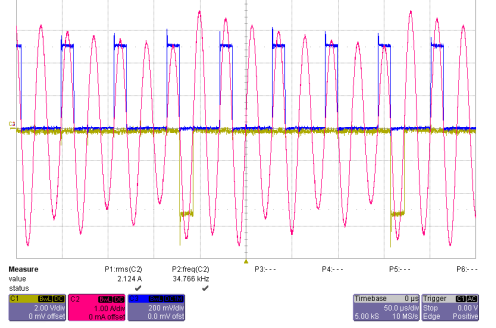

(g) Level 2-8

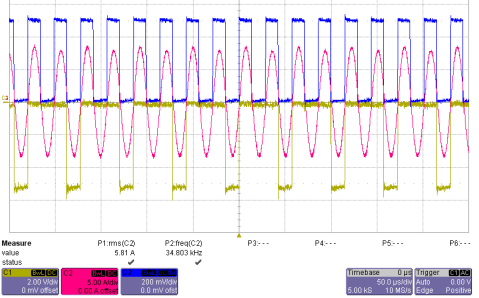

(b) Level 1-2

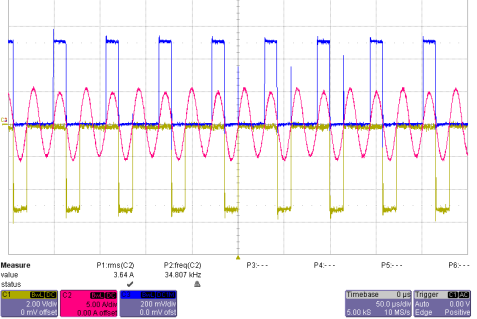

(e) Level 2-2

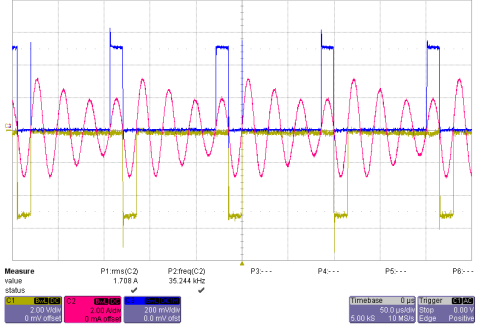

(h) Level 4-4

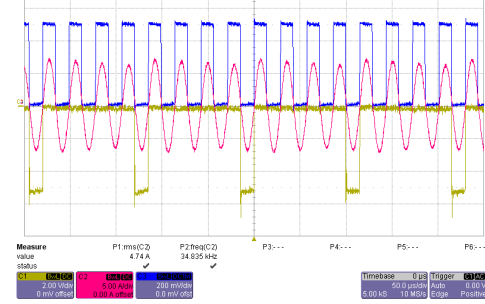

(c) Level 1-4

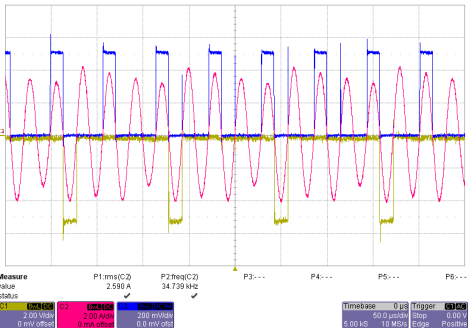

(f) Level 2-4

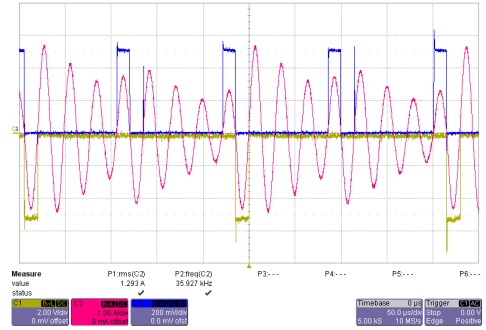

(i) Level 4-8

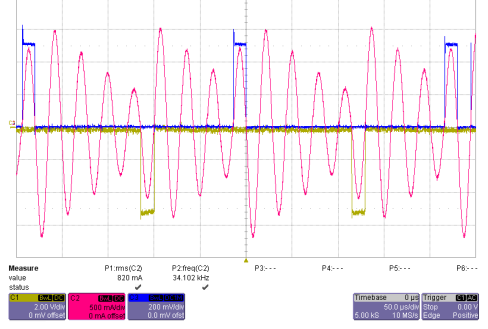

(j) Level 8-8

Figure 3.14: Experimental test results on the IPT system setup: the resonant current and energy-injection switching signals at different charging levels.

of the rectifier is connected to a three-phase power supply with a reduced line-to-line voltage of $25 \mathrm{~V}$, with $60 \mathrm{~Hz}$ frequency.

The proposed controller is tested at multiple charging levels according to Table 3.3 and the results are presented in Table 3.5. The resonant current and the energy 
Table 3.5: Experimental test results on the case study IPT system using the proposed controller at different charging levels.

\begin{tabular}{c|lllll}
\hline \hline \multirow{2}{*}{ Charging Level } & \multicolumn{2}{|c|}{ Frequency of energy injection } & & \\
\cline { 2 - 3 } & $\begin{array}{l}\text { Positive } \\
\text { cycles }\end{array}$ & $\begin{array}{l}\text { half- } \\
\text { cycles }\end{array}$ & $\begin{array}{l}\text { Resonant } \\
\text { current (A) }\end{array}$ & $\begin{array}{l}\text { Output } \\
\text { power (W) }\end{array}$ \\
\hline Level 1-1 & $f_{r}$ & $f_{r}$ & 8.00 & 165.76 \\
Level 1-2 & $f_{r}$ & $f_{r} / 2$ & 5.81 & 87.42 \\
Level 1-4 & $f_{r}$ & $f_{r} / 4$ & 4.74 & 58.19 \\
Level 1-8 & $f_{r}$ & $f_{r} / 8$ & 4.19 & 45.47 \\
Level 2-2 & $f_{r} / 2$ & $f_{r} / 2$ & 3.64 & 34.32 \\
Level 2-4 & $f_{r} / 2$ & $f_{r} / 4$ & 2.59 & 17.37 \\
Level 2-8 & $f_{r} / 2$ & $f_{r} / 8$ & 2.12 & 11.64 \\
Level 4-4 & $f_{r} / 4$ & $f_{r} / 4$ & 1.70 & 7.48 \\
Level 4-8 & $f_{r} / 4$ & $f_{r} / 8$ & 1.29 & 4.31 \\
Level 8-8 & $f_{r} / 8$ & $f_{r} / 8$ & 0.82 & 1.74 \\
\hline
\end{tabular}

injection switching signals for each charging level are shown in Fig. 3.14. This figure shows that due to the self-tuning capability of the converter, the switching operations are all synced with the resonant current (at $35 \mathrm{kHz}$ resonance frequency). Also, Fig. 3.14 verifies the ZCS operations which occur at the current zero-crossing points. Therefore, the proposed controller effectively self-tunes the switching operations with the resonant current and achieves soft-switching operations at each charging level.

\subsection{Development of a Power-Frequency Controller with Res- onance Frequency Tracking Capability for Inductive Power \\ Transfer Systems}

A self-tuning controller for power transfer regulation in inductive power transfer (IPT) systems is proposed in this section. The controller enables power transfer regulation around a user-defined reference power level. The converter's efficiency is improved by constantly tuning the switching operations to the resonant current, 
thereby achieving the soft-switching operations reducing the electromagnetic interference (EMI) the power converters. The self-tuning capability makes it ideal for dynamic IPT systems with uncertain loads and fluctuating resonance frequency. High operating frequencies can be achieved using the proposed simplified digital circuit design for the controller which delivers a low total propagation delay. Bidirectional power transfer can be enabled by using the proposed controller on both transmitter as well as receiver sides. In the reverse power flow mode, the primary converter operates as a rectifier and the power transfer is controlled through the secondary converter using the proposed controller. The performance of the proposed controller is analyzed using MATLAB/Simulink, and the results are presented. Finally, the proposed controller is implemented experimentally and its performance is evaluated as a case study on an IPT system. The experimental and simulation results conform to each other and show that the proposed converter can effectively regulate the power transfer with an improved efficiency.

This section presents design and implementation of a power-frequency controller that regulates the power transfer rate in accordance with the desired level (specified by the user) and tracks the resonance frequency of the system in a way that maximizes the power transfer efficiency. This controller also ensures its performance is not affected by system changes due to dynamic variations, as is the case in roadway IPT systems. Above all, the method proposed in this section simplifies the design and complexity of the controller, thereby reducing implementation costs. The existing methods in literature fall short in all of the above aspects, highlighting the uniqueness of the proposed controller. Although the proposed controller leverages similar benefits in terms of its self-tuning capability, low EMI, soft-switching operations, low switching stress, etc., its real novelty lies in the simplified, cost-effective design, and its ability to maximize power transfer efficiency even in dynamic system 
Table 3.6: Distinct power transfer levels and corresponding energy injection frequencies for positive and negative half-cycles.

\begin{tabular}{l|l|l}
\hline \hline \multirow{2}{*}{$\begin{array}{l}\text { Power } \\
\text { Level } n-m\end{array}$} & \multicolumn{2}{|c}{ Frequency of energy injection } \\
\cline { 2 - 3 } & $\begin{array}{l}\text { Positive half- } \\
\text { cycles }\left(f_{r} / n\right)\end{array}$ & $\begin{array}{l}\text { Negative half- } \\
\text { cycles }\left(f_{r} / m\right)\end{array}$ \\
\hline Level 1-1 & $f_{r}$ & $f_{r}$ \\
Level 1-2 & $f_{r}$ & $f_{r} / 2$ \\
Level 1-4 & $f_{r}$ & $f_{r} / 4$ \\
Level 1-8 & $f_{r}$ & $f_{r} / 8$ \\
Level 2-2 & $f_{r} / 2$ & $f_{r} / 2$ \\
Level 2-4 & $f_{r} / 2$ & $f_{r} / 4$ \\
Level 2-8 & $f_{r} / 2$ & $f_{r} / 8$ \\
Level 4-4 & $f_{r} / 4$ & $f_{r} / 4$ \\
Level 4-8 & $f_{r} / 4$ & $f_{r} / 8$ \\
Level 8-8 & $f_{r} / 8$ & $f_{r} / 8$ \\
\hline \hline
\end{tabular}

environments. A digital circuit-based design and implementation of the proposed controller is presented, which is capable of effectively regulating the transferred power. In order to validate the controller's efficacy, it is analyzed theoretically, simulated in MATLAB/Simulink, and then experimentally implemented, the results of which are discussed in detail.

\subsubsection{Proposed Control Method}

A control method based on variable frequency energy-injection and free-oscillation technique for IPT systems is presented in [162], which provides 10 power transfer levels (Table 3.6). These power levels are achieved by independently controlling the frequency of energy-injection into the IPT system at positive and negative halfcycles of the resonant current. The power transfer levels are labeled as $n-m$, where $n$ and $m$ correspond to $f_{r} / n$ and $f_{r} / m$ energy-injection frequencies for positive and negative half-cycles, respectively (where $f_{r}$ is the resonance frequency). Figure 3.16 conceptually shows the positive and negative half-cycle energy-injection signals for different power levels $(n, m \in\{1,2,4,8\})$. In this study, the control technique is further developed in order to design a closed-loop self-tuning controller that enables 
power transfer regulation in IPT systems. This is achieved by designing a control loop for power level that can regulate the power level around the reference power $\left(P_{\text {ref }}\right)$ by switching between energy-injection states presented in Table 3.6.

\subsubsection{Power-Frequency Controller}

The proposed controller along with a full-bridge DC/AC converter connected to an RLC is shown in Fig. 3.15. The RLC load represents the IPT system where $L$ is the self-inductance of the primary coil, $C$ is the series compensation capacitor, and $R_{e q}$ is the equivalent reflected resistance of the secondary. The controller takes the resonant current as feedback generates four output signals to control the fullbridge converter based on the reference power level $\left(P_{r e f}\right)$. The proposed controller

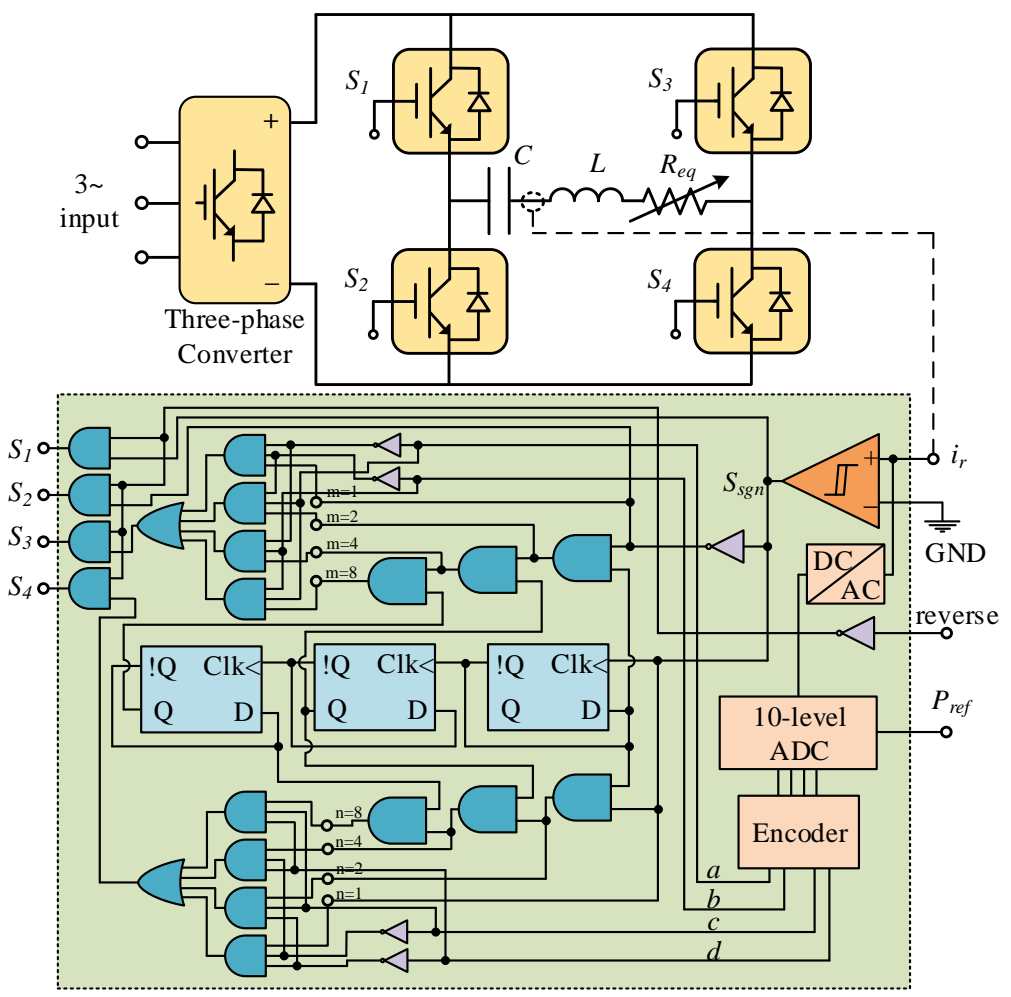

Figure 3.15: The proposed power-frequency controller designed based on a digital control circuit. 
is comprised of a differential comparator, AND, OR, NOT logic gates, flip-flops, signal rectifier, 10-level analog to digital converter (ADC) and an encoder.

Figure 3.16 conceptually shows the positive and negative half-cycle energy-injection signals for different power levels $(n, m \in\{1,2,4,8\})$ which are presented in Table 3.6 and the control circuit diagram shown in Fig. 3.15. These energy injection signals are generated using a three-stage frequency divider (three flip-flops connected in series) and are routed to the output switching signals using two 4-input multiplexer circuits (combination of 2 NOT gates, 4 AND gates, and an OR gate). The multiplexers are switched using four selectors ( $a$ and $b$ for negative half-cycles, and $c$ and $d$ for positive half-cycles) which are determined based on the resonant current amplitude and the reference power $\left(P_{\text {ref }}\right)$.

The circuit is designed to achieve zero-current switching by synchronizing the switching operations with the resonant current. This is done using the output of the resonant current zero-cross detector (output of the differential comparator, $S_{s g n}$ ) as a clock source signal for the resonance frequency divider. The frequency divider is used to change the energy injection frequency in order to control the power transfer level. A signal rectifier (AC/DC converter) is used to convert the measured $\mathrm{AC}$ resonant current into a DC signal in order to enable comparisons with the reference level. This signal determines the level of the resonant current. Using an ADC, the corresponding DC signal is then converted to a 4-digit signal by comparing the equivalent DC signal to the reference level input. Based on the level of the resonant current, the encoder generates 4 signals to control the power transfer level and minimize the power level error respect to the reference power level. The power transfer control is achieved by tuning the frequency of energy injection to the IPT systems using two multiplexer circuits for positive and negative half-cycle energy injection signals, which are generated by the resonance frequency divider circuits. 


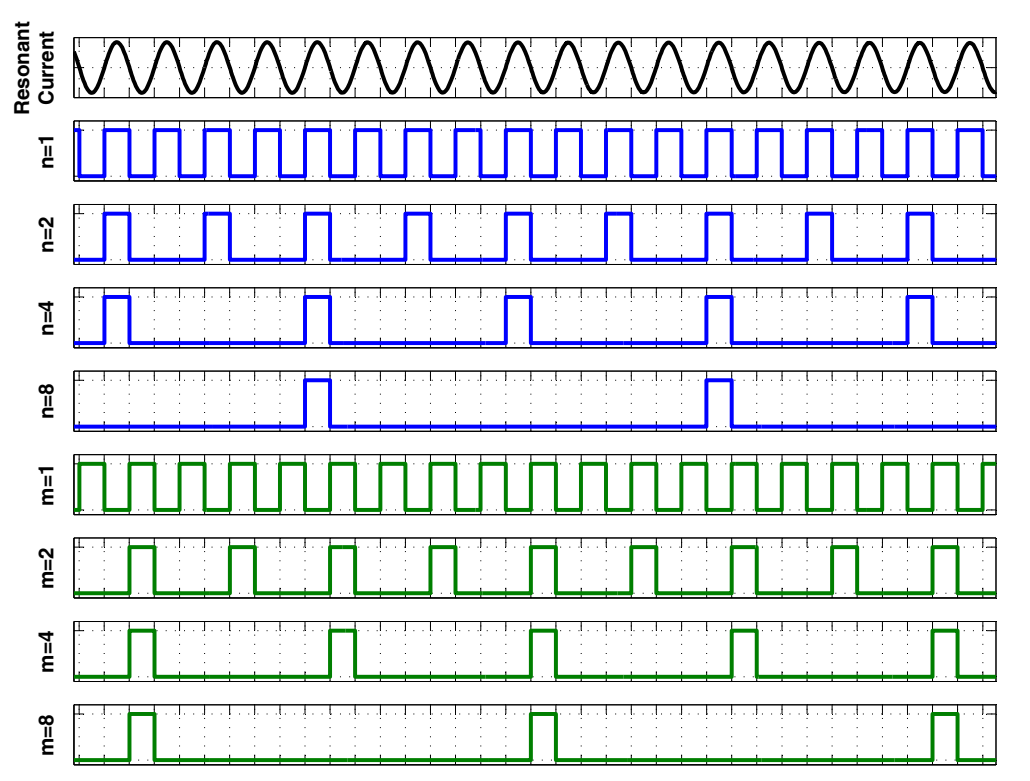

Figure 3.16: Resonant current and corresponding positive and negative half-cycle energy injection signals for $n, m \in\{1,2,4,8\}$.

The "reverse" input is used to enable reverse power flow mode by disabling all the switching signals and thus allowing the converter to operate as a rectifier. In this case, the secondary coil works as a transmitter and the primary coil works as a receiver allowing a reverse power flow. If the primary circuit is powered by an $\mathrm{AC}$ source, an inverter is required between the $\mathrm{AC}$ power source and the DC-link in order to enable power transfer to the grid, forming a V2G connection.

\subsubsection{Self-Tuning Capability}

The power electronic converters used in IPT systems should operate at the resonance frequency of the system in order to achieve the maximum power transfer efficiency. Any deviation from the resonance frequency can dramatically affect the performance of the system. Due to different operating conditions in IPT systems such as, transmitter and receiver alignment, load characteristics, etc., the characteristics of the system including resonance frequency may change. Also, different 


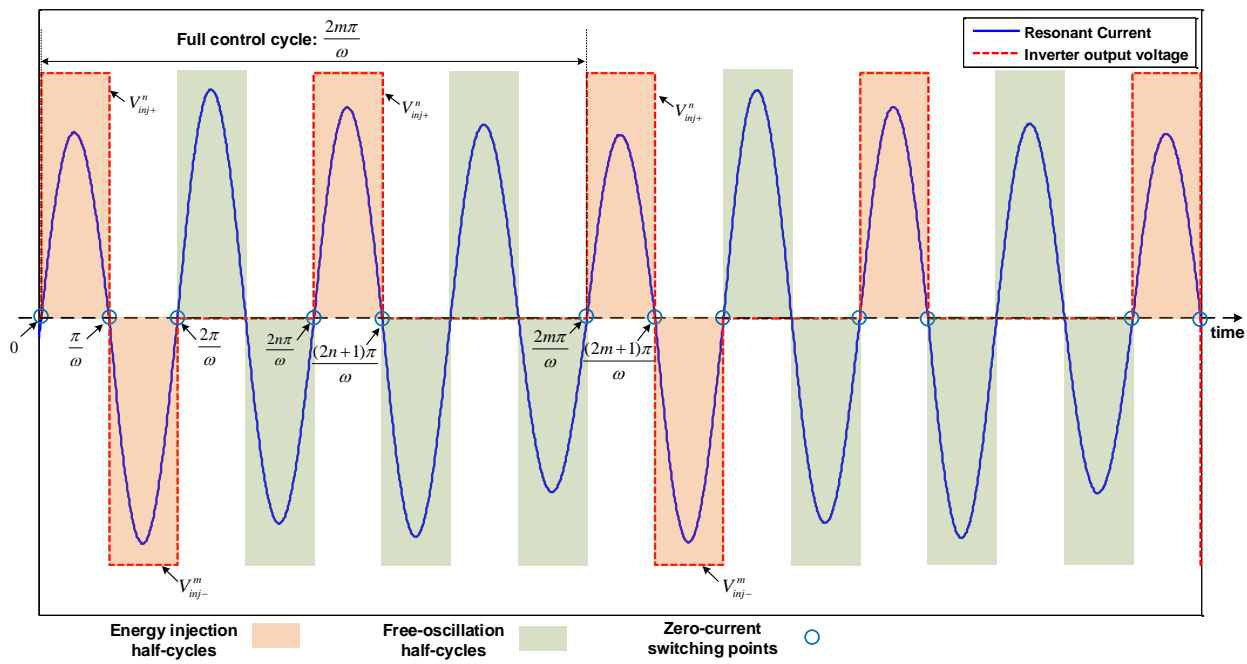

Figure 3.17: The resonant current and corresponding switching signals controlled using the proposed controller for power level 2-4 $(n=2, m=4)$.

IPT systems can be designed based on a range of operating resonance frequencies (e.g. $20-85 \mathrm{kHz}$ for inductive EV charging systems). Furthermore, IPT systems can have a variable resonance frequency for different purposes [159]. Therefore, the use of self-tuning controllers that can track and tune the switching operations of the converters with the resonance frequency of the IPT system would be of great interest, as it eliminates the need for manual tuning and it can significantly enhance the performance of the system.

The proposed controller (Fig. 3.15) synchronizes the switching signals with the resonant current of the IPT system to enable the self-tuning capability. This is achieved by using the resonant current sign signal $\left(S_{\text {sgn }}\right.$ determined by the differential comparator), and its complement $\overline{S_{s g n}}$ as clock sources for the entire control circuit. This ensures that the switching operations always occur at the resonant current zero-crossing points, and therefore, the converter is switched at the resonance frequency. This also enables zero-current switching operations which significantly enhance the performance of the converter and reduce switching stress. 


\subsubsection{Theoretical Analysis}

The proposed power controller achieves power transfer control based on resonant current regulation. Therefore, finding an analytical solution for the resonant current in an IPT system would be useful in order to calculate the power transfer level at different energy injection frequencies.

\subsubsection{Power Transfer Level}

In an RLC circuit shown in Fig. 3.15, the equation for the resonant current can be written as follows:

$$
L \frac{d i}{d t}+\frac{1}{C} \int i d t+R_{e q} i=V_{i n j+}^{n}(t)+V_{i n j-}^{m}(t)
$$

where $i$ is the resonant current, $V_{i n j+}^{n}(t)$ and $V_{i n j-}^{m}(t)$ are output voltage pulses of the converter at energy injection level $n-m$ in positive and negative half-cycles respectively. In Fig. 3.17, $V_{i n j+}^{n}(t)$ and $V_{i n j-}^{m}(t)$ for power level $2-4(n=2$ and $m=4)$ are conceptually shown. These input functions are periodic with cycles of $2 n \pi / \omega$ and $2 m \pi / \omega$ ( $\omega$ is the frequency of the resonant current), and can be expressed as,

$$
\begin{gathered}
V_{i n j+}^{n}(t)= \begin{cases}V_{t} & 0<t<\frac{\pi}{\omega} \\
0 & \frac{\pi}{\omega}<t<\frac{2 n \pi}{\omega}\end{cases} \\
V_{i n j-}^{m}(t)= \begin{cases}-V_{t} & \frac{\pi}{\omega}<t<\frac{2 \pi}{\omega} \\
0 & 0<t<\frac{\pi}{\omega}, \frac{2 \pi}{\omega}<t<\frac{2 m \pi}{\omega}\end{cases}
\end{gathered}
$$

Equation (3.23) can be solved by applying the superposition law and considering $V_{i n j+}^{n}(t)$ and $V_{i n j-}^{m}(t)$ as two separate input functions:

$$
L \frac{d i_{1}}{d t}+\frac{1}{C} \int i_{1} d t+R_{e q} i_{1}=V_{i n j+}^{n}(t)
$$




$$
L \frac{d i_{2}}{d t}+\frac{1}{C} \int i_{2} d t+R_{e q} i_{2}=V_{i n j-}^{m}(t)
$$

where $i_{1}$ and $i_{2}$ are corresponding solutions due to $V_{i n j+}^{n}(t)$ and $V_{i n j-}^{m}(t)$ input functions respectively. First, the analytical solution for $i_{1}$ is found. Since in each halfcycle $V_{i n j+}^{n}(t)$ is constant, $(3.26)$ can be rewritten as follows:

$$
\frac{d^{2} i_{1}}{d t^{2}}+\frac{R_{e q}}{L} \frac{d i_{1}}{d t}+\frac{1}{L C} i_{1}=0
$$

The controller is designed in way that zero-current switching is obtained. Therefore, the initial conditions would be as follows:

$$
i_{1}(0)=0, \quad L \frac{d i_{1}}{d t}(0)=V_{i n j+}^{n}(t)-v_{c}(0)
$$

where $V_{i n j+}^{n}(t)$ is the converter output voltage and $v_{c}(0)$ is the initial capacitor voltage. Therefore, the solution of (3.28) would be as follows:

$$
i_{1}=\frac{V_{i n j+}^{n}-v_{c}(0)}{\omega L} e^{-t / \tau} \sin (\omega t)
$$

where $\omega=\sqrt{\omega_{0}^{2}-1 / \tau^{2}}$ is the natural resonance frequency, $\omega_{0}=1 / \sqrt{L C}$ is the resonance frequency, and $\tau=2 L / R_{e q}$ is the damping time constant. Assuming that the system has reached to a steady-state condition, $v_{c}(0)$ can be calculated as follows [162]:

$$
v_{c}(0)=-\frac{(1+\beta)}{1-\beta^{2 n}} \beta^{2 n-1} V_{t}
$$

where $\beta=e^{\frac{-\pi}{\tau \omega}}$. Using (3.24), (3.30), and (3.31), $i_{1}$ can be derived as follows:

$$
i_{1}(t)= \begin{cases}\frac{1+\beta^{2 n-1}}{\omega L\left(1-\beta^{2 n}\right)} V_{t} e^{-t / \tau} \sin (\omega t) & 0<t<\frac{\pi}{\omega} \\ \frac{(1+\beta) \beta^{2 n-1}}{\omega L\left(1-\beta^{2 n}\right)} V_{t} e^{-t / \tau} \sin (\omega t) & \frac{\pi}{\omega}<t<\frac{2 n \pi}{\omega}\end{cases}
$$


Since negative half-cycle energy injection always happens after a positive halfcycle energy injection, the following can be obtained:

$$
V_{i n j-}^{m}(t)=-V_{i n j+}^{m}\left(t-\frac{\pi}{\omega}\right)
$$

Since (3.26) is linear and time-invariant, based on (3.33) the following is obtained:

$$
i_{2}(t)=-\left.i_{1}\left(t-\frac{\pi}{\omega}\right)\right|_{n=m}
$$

In order to eliminate the power levels that will result in a repetitive power level, the proposed controller is designed in a way that $n \leq m$. Using (3.34) and (3.32), $i_{2}$ can be written as follows:

$$
i_{2}(t)=\left\{\begin{array}{cl}
\frac{(1+\beta) \beta^{2 m-2}}{\omega L\left(1-\beta^{2 m}\right)} V_{t} e^{-t / \tau} \sin (\omega t) & 0<t<\frac{\pi}{\omega} \\
\frac{1+\beta^{2 m-1}}{\omega L\left(1-\beta^{2 m}\right) \beta} V_{t} e^{-t / \tau} \sin (\omega t) & \frac{\pi}{\omega}<t<\frac{2 m \pi}{\omega}
\end{array}\right.
$$

Applying the superposition principle for differential equations, the final solution for the resonant current can be expressed as follows:

$$
i=i_{1}+i_{2}
$$

Using the analytical solution for the resonant current, the transferred power can be obtained as follows:

$$
P_{i n j}=\frac{\int_{0}^{\frac{2 m \pi}{\omega}}\left(V_{i n j+}^{n}(t)+V_{i n j-}^{m}(t)\right) i(t) d t}{2 m \pi / \omega}
$$

Considering the fact that in a full cycle $(0<t<2 m \pi / \omega)$ there would be $m / n$ positive half-cycle energy-injections and only one negative half-cycle energyinjection, (3.37) can be rewritten as follows:

$$
P_{i n j}=\frac{\sum_{i=1}^{m / n} \int_{2(i-1) \pi / \omega}^{(2 i-1) \pi / \omega} V_{d c} i(t) d t+\int_{\pi / \omega}^{2 \pi / \omega}-V_{d c} i(t) d t}{2 m \pi / \omega}
$$


Using (3.32) and (3.35), (3.38) the power transfer level can be calculated based on IPT system parameters $L, C, R_{e q}$, DC-link voltage $V_{d c}$, and power transfer level $n-m$. This equation is specifically useful for estimating the output power at different power transfer levels given in Table 3.6.

\subsubsection{Voltage Gain of the Converter}

Since the proposed controller tunes the output voltage of the converter by switching to different modes of operation, its voltage gain characteristic would be of great interest. The RMS output voltage at power transfer level $n-m$ can be calculated as follows:

$$
V_{r m s}=\sqrt{\frac{\int_{0}^{2 m \pi / \omega} V_{t}^{2} d t}{2 m \pi / \omega}}
$$

Based on Fig. 3.17, (3.39) can be rewritten as,

$$
V_{r m s}=\sqrt{\frac{\sum_{i=1}^{m / n} \int_{2(i-1) \pi / \omega}^{(2 i-1) \pi / \omega} V_{d c}^{2} d t+\int_{\pi / \omega}^{2 \pi / \omega} V_{d c}^{2} d t}{2 m \pi / \omega}}
$$

Equation (3.40) can be simplified as follows:

$$
V_{r m s}=\sqrt{\frac{(m / n+1) V_{d c}^{2} \pi / \omega}{2 m \pi / \omega}}=\sqrt{\frac{n+m}{2 n m}} V_{d c}
$$

Using (3.41) the voltage gain of the converter can be obtained as follows:

$$
G_{v}=\frac{V_{r m s}}{V_{d c}}=\sqrt{\frac{n+m}{2 n m}}
$$

According to (3.42), the converter using the proposed controller operates as a controlled voltage source for the primary with control parameters $n$ and $m$. In IPT systems (specifically dynamic IPT systems), $R_{e q}$ can change due to inherent variations of the system such as vehicle alignment relative to primary and number of charging vehicles. The controller is designed to regulate the voltage gain with the 
variations of $R_{e q}$ to achieve the desired power transfer level $\left(P_{r e f}\right)$. For instance, if $R_{e q}$ decreases, output voltage of the converter is reduced ( $n$ and/or $m$ are increased) to regulate the power level around $P_{\text {ref }}$. In other words, lower $R_{e q}$ results in lower energy-injection levels (higher $n$ and/or $m$ ). At a fixed energy injection level (no power level regulation), the RMS output voltage of the converter is constant, therefore there would be an inverse relationship between power level and $R_{e q}$.

\subsubsection{System Response Time}

Since an IPT system can be represented with an equivalent RLC circuit (shown in Fig. 3.15), the settling time of the system within $2 \%$ of the final value can be expressed as follows:

$$
T_{s} \simeq 4 \tau=\frac{8 L}{R_{e q}}
$$

Equation (1) shows that there is an inverse relationship between response time of the system and $R_{e q}$. The relationship between power level and different system parameters including $R_{e q}$ is presented in (3.38).

\subsubsection{Steady-State Error}

As discussed in Section 3.3.1, the proposed controller operates based on 10 discrete power transfer levels presented in Table 3.6. This means that the controller switches between these discrete power levels to minimize the output power error. In Fig. 3.18, the output power and steady-state error are plotted as a function of reference power. The output power (P.U.) is calculated using (3.38) at different energy injection levels and the relationship between the output power and the reference power (P.U.)

is determined based on the design of the controller. Figure 3.18 shows that the maximum steady-state error of the system is $22 \%$. 


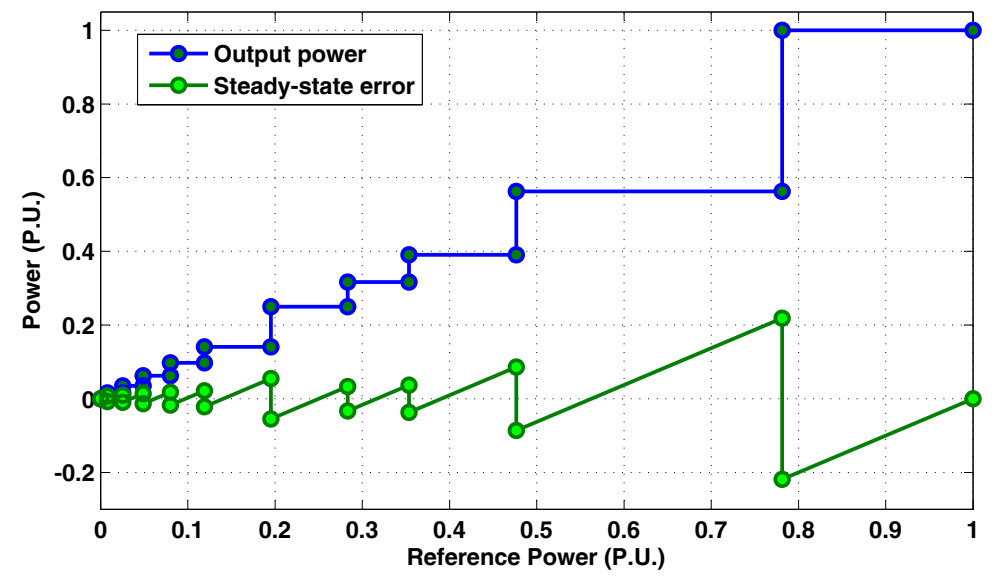

Figure 3.18: The output power and steady-state error as a function of reference power.

\subsubsection{Simulation Results}

The proposed controller along with an IPT system is modeled and simulated in MATLAB/Simulink using SimPowerSystem toolbox and the performance of power transfer regulation is evaluated at different conditions. The simulation model and the specifications of the IPT system are presented in Fig. 3.19 and Table 3.7 respectively. The IPT system is comprised of a three-phase two-stage AC/DC/AC converter connected to the primary circuit, magnetic couplers, compensation capacitors, secondary AC/DC converter which charges an EV battery. The power controller is modeled based on the control circuit presented in Fig. 3.15 using AND, OR and NOT logic gates, flip-flops, ADC conversion. The voltage comparator is modeled as a "compare to zero" block which acts as zero-cross detector. The current measurement transformer is modeled as a controlled current source with a 1:1000 conversion ratio. The current measurement signal is converted into a DC signal using a half-wave rectifier which is then connected to the ADC/Encoder block in order to switch the controller to the appropriate power transfer level. The controller generates the switching signals of the second stage (inverter stage) of the primary 


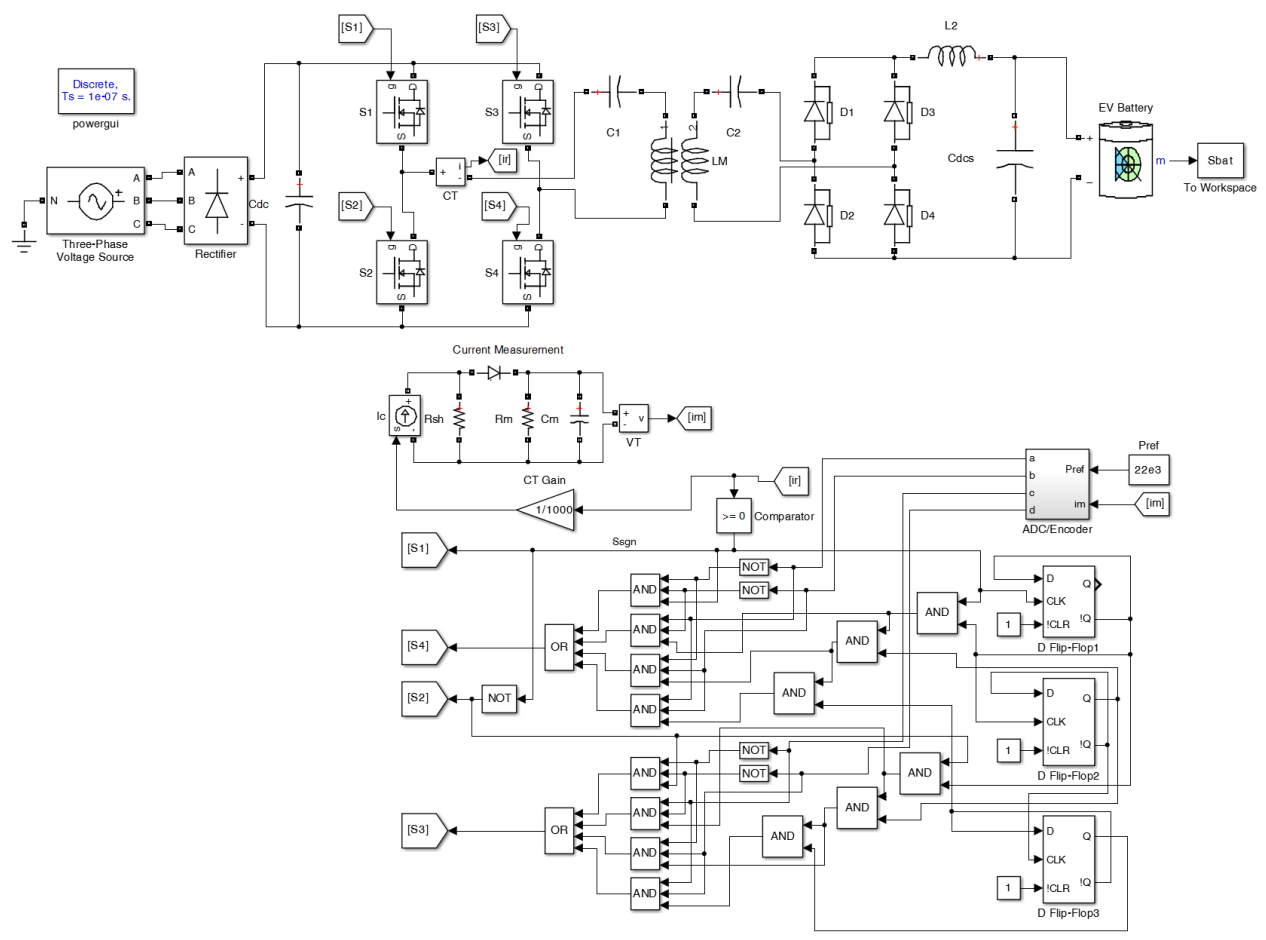

Figure 3.19: The case study IPT system simulation model.

Table 3.7: Specifications of the case study IPT system.

\begin{tabular}{c|c}
\hline \hline Parameter & Value \\
\hline Primary and secondary self-inductances & $172 \mu \mathrm{H}$ \\
Primary and secondary compensation capacitors & $120 \mathrm{nF}$ \\
Resonance frequency & $35 \mathrm{kHz}$ \\
Grid line voltage & $208 \mathrm{~V}$ \\
EV battery voltage & $360 \mathrm{~V}$ \\
EV battery capacity & $22 \mathrm{kWh}$ \\
\hline \hline
\end{tabular}

converter. The reference power level is determined using (3.38), based on the energy injection levels for positive and negative half-cycles $(n$ and $m)$.

The simulations are carried out for all power levels according to Table 3.6, and the results are presented in Table 3.8. The results include grid power and current (rms), battery charging power and current, and efficiency of the system. Also, the power level and efficiency of the system at different power levels are presented in Fig. 3.20 which shows that as the power level drops (higher $n$ and/or $m$ ), the 
Table 3.8: Distinct power transfer levels and corresponding energy injection frequencies for positive and negative half-cycles.

\begin{tabular}{l|ll|ll|l}
\hline \hline $\begin{array}{l}\text { Power } \\
\text { Level } \\
n-m\end{array}$ & $\begin{array}{l}\text { Grid } \\
\text { power } \\
(\mathrm{kW})\end{array}$ & $\begin{array}{l}\text { Grid } \\
\text { cur- } \\
\text { rent } \\
(\mathrm{A})\end{array}$ & $\begin{array}{l}\text { Battery } \\
\text { charging } \\
\text { current } \\
(\mathrm{A})\end{array}$ & $\begin{array}{l}\text { Battery } \\
\text { charging } \\
\text { power } \\
(\mathrm{kW})\end{array}$ & $\begin{array}{l}\text { Efficiency } \\
(\%)\end{array}$ \\
\hline $1-1$ & 33.83 & 112.65 & 92.61 & 33.34 & 98.54 \\
$1-2$ & 23.28 & 89.56 & 63.28 & 22.75 & 97.72 \\
$1-3$ & 18.76 & 78.97 & 50.81 & 18.21 & 97.07 \\
$1-4$ & 16.60 & 73.76 & 45.17 & 16.06 & 96.77 \\
$2-2$ & 14.51 & 68.33 & 39.38 & 13.97 & 96.27 \\
$2-3$ & 10.48 & 56.98 & 28.14 & 9.97 & 95.17 \\
$2-4$ & 8.55 & 50.80 & 22.66 & 8.04 & 94.08 \\
$3-3$ & 6.65 & 44.38 & 17.04 & 6.18 & 92.87 \\
$3-4$ & 4.80 & 36.94 & 12.80 & 4.37 & 91.01 \\
$4-4$ & 3.00 & 28.16 & 7.95 & 2.63 & 87.75 \\
\hline \hline
\end{tabular}

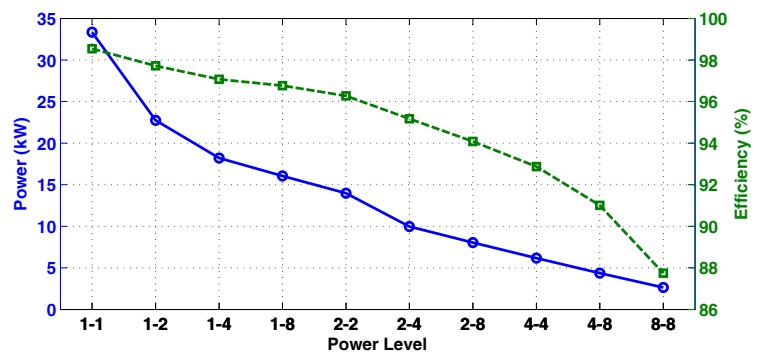

Figure 3.20: Power and efficiency of the converter at different power transfer levels calculated based on simulation results.

efficiency of the converter drops. It is due to the fact that at lower power levels, the number of free-oscillation cycles are increased by the controller, in order to reduce energy injection into the IPT system, thereby allowing the resonant current to freely oscillate through the switches of the converter. This in turn increases conduction losses in the converter. Figure 3.20 also shows that for the first five power levels (levels 1-1, 1-2, 1-4, 2-2, 2-4), the converter can achieve a minimum efficiency of 96\%. Furthermore, the maximum efficiency that is achieved at level 1-1 with 33.83 $\mathrm{kW}$ power is $98.54 \%$.

By applying step changes in the reference power level, the performance of the controller is analyzed in tracking the reference power level. In Fig. 3.21, the simula- 


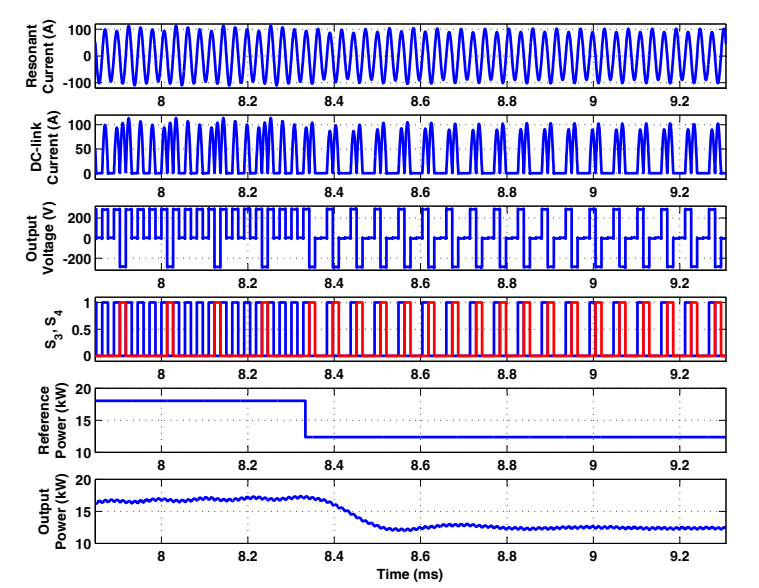

(a) Transition from Level $1-4(18 \mathrm{~kW})$ to Level 2-2 $(12 \mathrm{~kW})$.

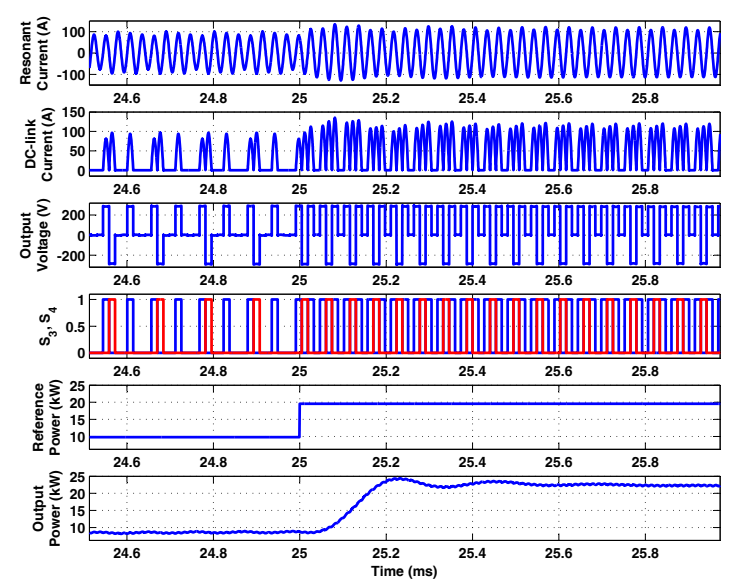

(b) Transition from Level $2-4(10 \mathrm{~kW})$ to Level 1-2 $(20 \mathrm{~kW})$.
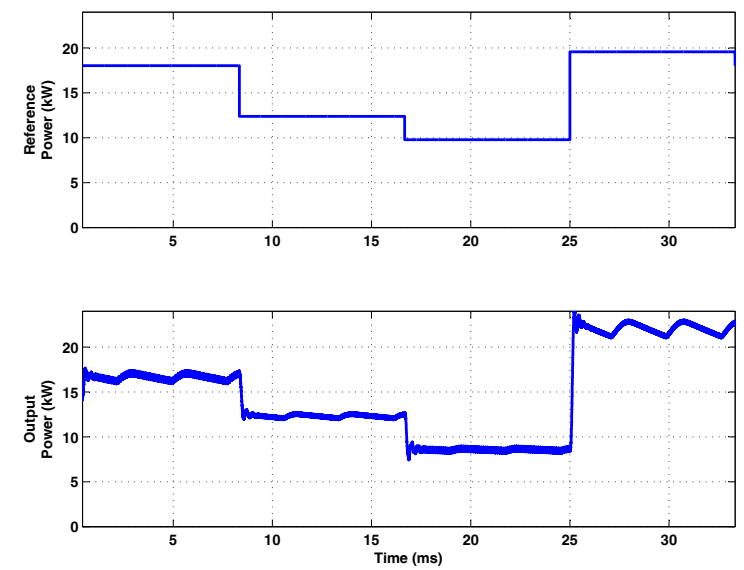

(c) Transition from Level 1-4 $(18 \mathrm{~kW})$, Level 2-2 (12 kW), Level 2-4 (10 kW) and Level 1-2 $(20 \mathrm{~kW})$.

Figure 3.21: Simulation results on the case study IPT system showing resonant current, DC-link current, energy-injection switching signals $\left(S_{3}, S_{4}\right)$, reference power and output power.

tion results including resonant current, DC-link current, energy-injection switching signals, reference power and output power are presented for different power level transitions. The output power is calculated based on the power delivered to batteries at the secondary circuit. Figure 3.21(a) shows the transition from level 1-4 (18 $\mathrm{kW})$ to level 2-2 (12 kW). In Fig. 3.21(b) transition from level 2-4 (10 kW) to level 


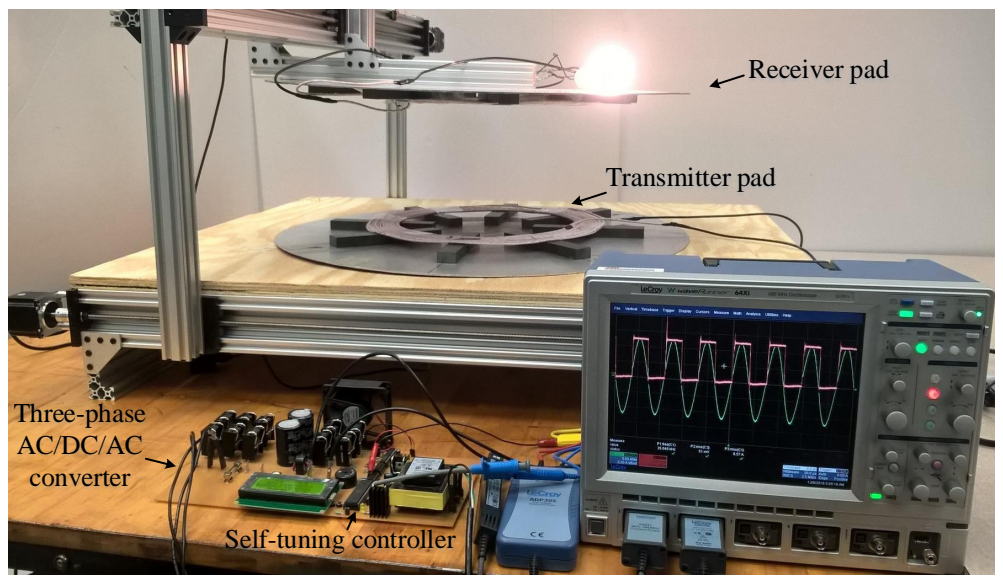

Figure 3.22: The experimental set-up of the proposed power-frequency controller.

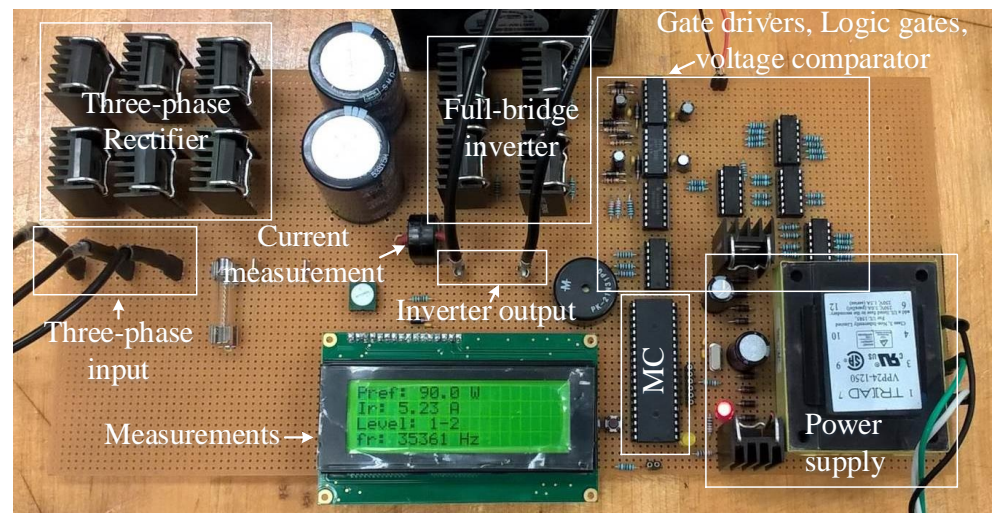

Figure 3.23: Experimental setup of the proposed controller and its components.

1-2 (20 kW). Also, in Fig. 3.21(c) transition from level 1-4 (18 kW), level 2-2 (12 $\mathrm{kW})$, level 2-4 (10 kW) and level 1-2 $(20 \mathrm{~kW})$ are presented. The results show that the using the proposed controller, the output power of the IPT system effectively tracks the reference current (estimated output power) with low discrepancy. The controller effectively changes the energy injection frequencies in order to conform to the reference power level. In Figs. 3.21(a) and (b),it can be seen that the switching operations always occur at the resonant current zero-crossing points which verifies the self-tuning capability of the controller and zero-current switching (ZCS) in the converter described earlier in Section 3.3.1.2. 
Table 3.9: Specifications of the experimental IPT system.

\begin{tabular}{c|c|c}
\hline \hline Parameter & Components & Value \\
\hline Pad self-inductance & 15 turns, 10 AWG Litz wire & $172 \mu \mathrm{H}$ \\
Compensation capacitors & Film capacitor, FPG66Y0124J & $120 \mathrm{nF}$ \\
Primary supply & Variable three-phase AC supply & $10 \mathrm{~V}(\mathrm{LL}), 60 \mathrm{~Hz}$ \\
Secondary battery & Lead-acid battery & $12 \mathrm{~V}, 86.4 \mathrm{Wh}$ \\
\hline \hline
\end{tabular}

Table 3.10: List of components used in experimental implementation of the proposed power controller.

\begin{tabular}{c|c}
\hline \hline Component & Part number \\
\hline Switches $\left(S_{1}, S_{2}, S_{3}, S_{4}\right)$ & CREE CMF20120D \\
Gate driver & IR2110 \\
Current transducer & PE-51688 \\
Differential comparator & TLC374 \\
NOT gate & SN74LS04 \\
AND gate & SN74HC08 \\
OR gate & M74HC4072 \\
Flip-flop & SN74LS74 \\
ADC, Encoder & Atmel ATmega32 \\
\hline \hline
\end{tabular}

\subsubsection{Experimental Results}

In order to validate the effectiveness of the proposed control circuit for controlling the power transfer in IPT system, experimental test results were carried out on a case study IPT system. The case study system which is shown in Fig. 3.22, is composed of two circular transmitter and receiver magnetic structures, primary three-phase converter, compensation capacitors, and a battery charger connected to a $12 \mathrm{~V}$ battery at the receiver side. The primary converter is a two-stage $\mathrm{AC} / \mathrm{DC} / \mathrm{AC}$ converter where its first stage is a full-bridge three-phase AC/DC converter connected to a $60 \mathrm{~Hz}$ variable three-phase source and the second stage is a full-bridge single-phase $\mathrm{DC} / \mathrm{AC}$ converter which is controlled with the proposed power controller. The specifications of the circular power pads, compensation capacitors, primary AC supply and secondary battery conform to the ones given in Table 3.9. Resonance frequency of the IPT system is $35 \mathrm{kHz}$. The proposed control circuit which is presented in Fig. 3.15 is implemented experimentally and is shown in Fig. 3.23. Also, the circuit 


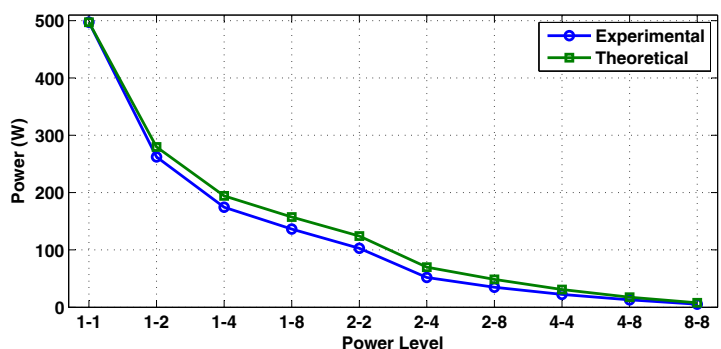

(a)

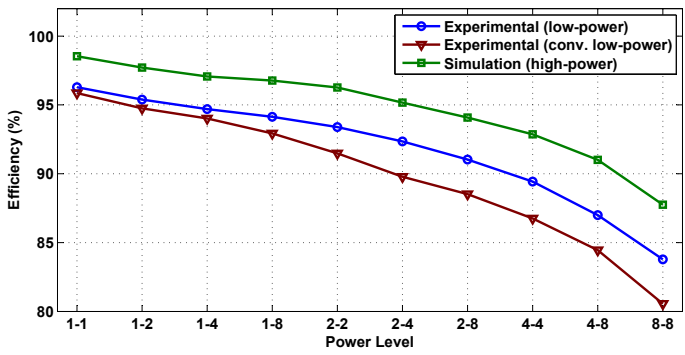

(b)

Figure 3.24: Experimental results on the case study IPT system at different power levels: (a) output power obtained based on experimental measurements and theoretical calculations, (b) converter efficiency obtained based on experimental measurements and simulation results.

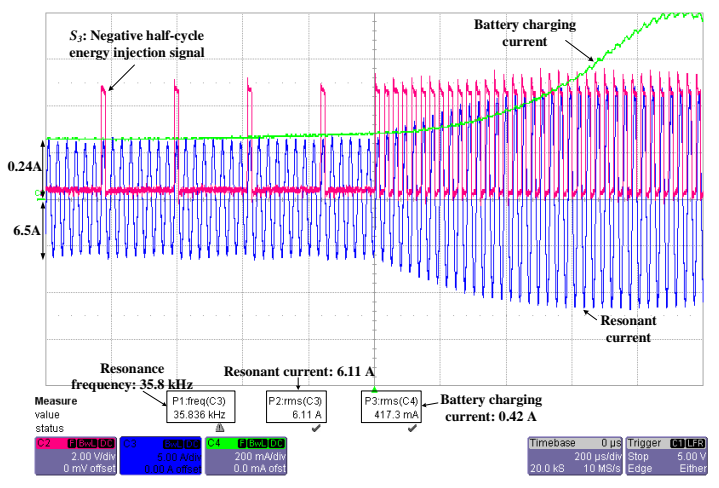

(a)

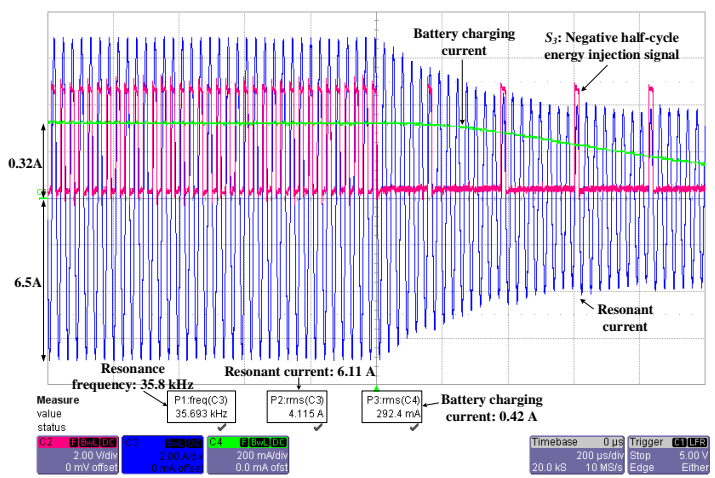

(b)

Figure 3.25: Experimental results on the IPT setup showing the resonant current, battery charging current and negative half-cycle switching signal $\left(S_{3}\right)$ : (a) transition from Level 1-8 to Level 1-1, (b) transition from Level 1-1 to Level 1-8.

components used in building the controller and converter prototype are listed in Table 3.10. The logic gates the differential comparator is used to detect resonant current zero-crossing points. The logic gates and flip-flops are utilized to build the three-stage resonance frequency divider and 4-input multiplexers (described in Section 3.3.1.1). A 10 level ADC (Analog-to-Digital Conversion) along with an encoder are implemented using an Atmel Atmega32 microcontroller. 


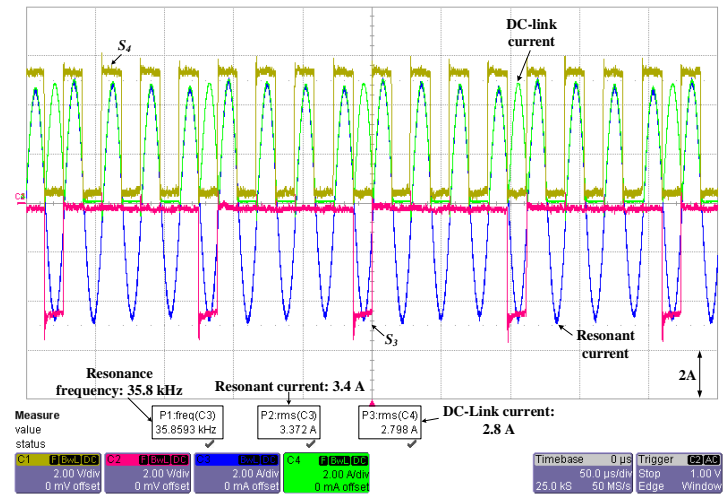

(a)

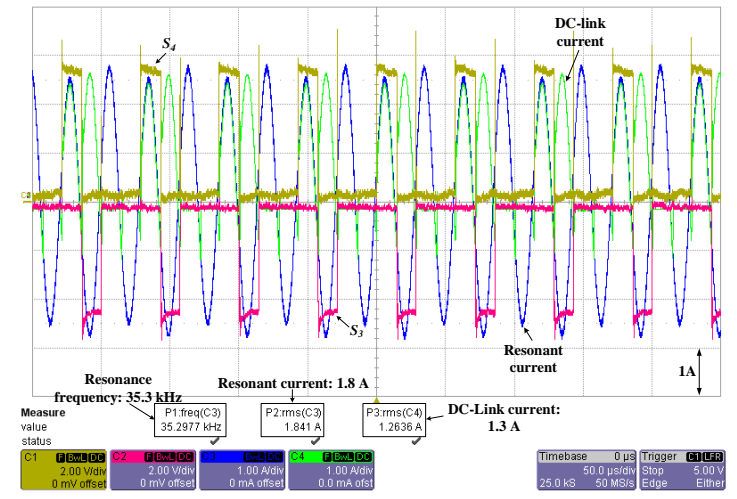

(b)

Figure 3.26: Experimental results on the IPT setup showing the resonant current, primary DC-link current and energy injection switching signals $\left(S_{3}\right.$ and $\left.S_{4}\right)$ : (a) Level 1-4 power transfer, (b) Level 2-2 power transfer.

The performance of the controller is evaluated by applying step changes in the reference power level which causes changes in the energy injection frequencies. In Figs. 3.25(a) and (b), the resonant current, battery charging current and negative half-cycle energy injection signals $\left(S_{3}\right)$ during the transitions from level 1-8 to level 1-1 and from level 2-1 to level 2-8 are shown. These figures show that the controller can effectively regulate the battery charging current and thereby the power transfer level according to the reference power level. Also, in Figs. 3.26(a) and (b), the resonant current, primary DC-link current and energy injection switching signals $\left(S_{3}\right.$ and $\left.S_{4}\right)$ for level 1-4 and level 2-2 power are shown. These figures show that the converter achieves ZCS by self-tuning of the switching operations to the resonant current. Experimental results of the converter output voltage (switch node voltage) and the resonant current at power Level 2-4 are presented in Fig. 3.27. This figure shows that the converter achieves Zero Current Switching (ZCS) operations. In free-oscillation modes, the output voltage equals to the voltage drop across two conducting switches $\left(S_{1}\right.$ and $S_{3}$, or $S_{2}$ and $\left.S_{4}\right)$ consisting of a body diode and a forward biased switch. In energy injection modes, the output voltage equals to $V_{d c}$ 
or $-V_{d c}$, incorporating the voltage drops across two conducting switches $\left(S_{1}\right.$ and $S_{4}$, or $S_{2}$ and $\left.S_{3}\right)$.

\subsubsection{Efficiency Analysis}

The power transfer at different levels with a maximum of $500 \mathrm{~W}$ calculated based on the experimental measurements on the case study IPT system setup and theoretical calculations, is presented in Fig. 3.24(a). This figure shows that the experimental measurements of power transfer at different power levels conform to the theoretical calculations with a small discrepancy. In 3.24(b), experimental efficiency measurements on the IPT system setup using both the proposed power controller and conventional controller at different power transfer levels are presented and compared to the simulation results at high-power levels. The conventional controller is designed based on the firing angle control with respect to resonant current zero-crossing points in order to regulate the resonant current and the power transfer rate. The figure shows that for low power rates $(500 \mathrm{~W})$, the converter can achieve a power transfer efficiency of $96 \%$ using both control methods. However, as the power level is decreased, the proposed power control method performs better and achieves higher efficiencies. This is due to the fact that the proposed power control method benefits from soft-switching operations. Also, based on Fig. 3.24(b), it can be seen that the converter achieves efficiencies above $98 \%$ at high-power levels (at level 1-1) which is only about $2 \%$ higher than efficiency measurements at low power level.

In Fig. 3.24(b), experimental efficiency measurements on the IPT systems setup using both the proposed power controller and conventional controllers at different power transfer levels are presented and are compared to the simulation results at

high-power levels. The conventional controller is designed based on switching firing angle control respect to the resonant current zero-crossing points to regulate the 


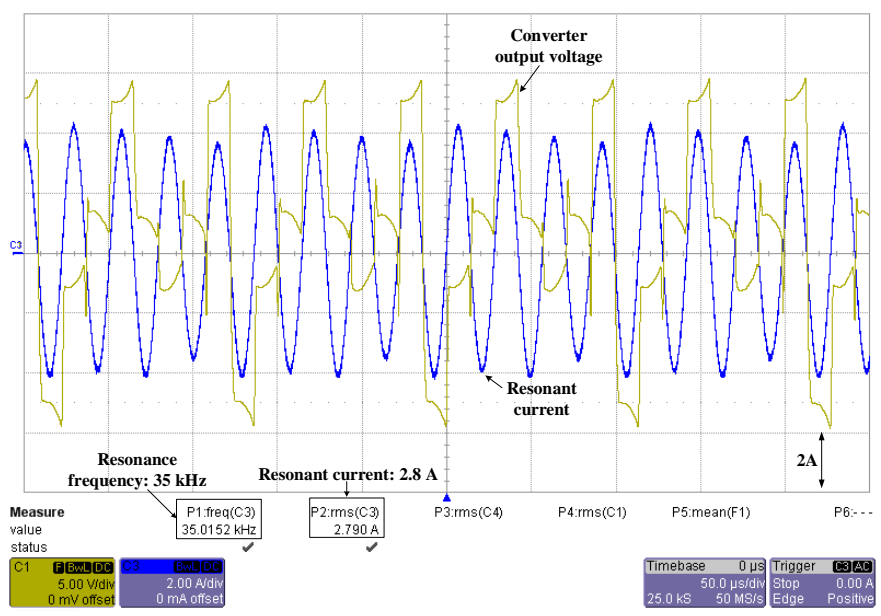

Figure 3.27: The output voltage of the $\mathrm{DC} / \mathrm{AC}$ converter and resonant current at power Level 2-4.

resonant current and the power transfer rate. The figure shows that for low power rates $(500 \mathrm{~W})$, using both control methods, the converter can achieve a power transfer efficiency of $96 \%$. But as the power level is decreased, the proposed power control method performs better and achieves higher efficiencies. This is due to the fact that the proposed power control method benefits from soft-switching operations. Also, based on Fig. 3.24(b), it can be seen that the converter achieves efficiencies above $98 \%$ at high-power levels (at level 1-1) which is only about $2 \%$ higher than efficiency measurements at low power level.

\subsubsection{Resonance Frequency Tracking Capability Analysis}

In order to verify the resonance frequency tracking capability of the controller, the case study IPT system is investigated under different transmitter and receiver pad alignments (vertical and horizontal). Any change in the pad alignment will lead to a change in the characteristics of system including the resonance frequency. The alignment of the IPT system is controlled using a multi-axis alignment system (shown in Fig. 3.22). The resonance frequency measurements are carried at with 


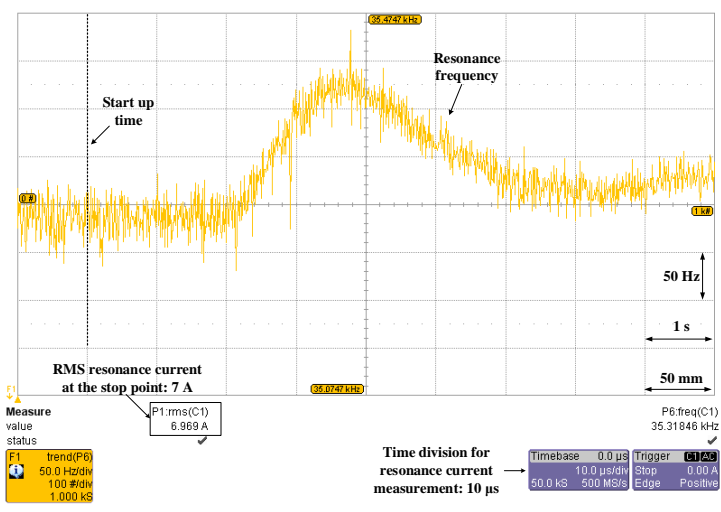

(a)

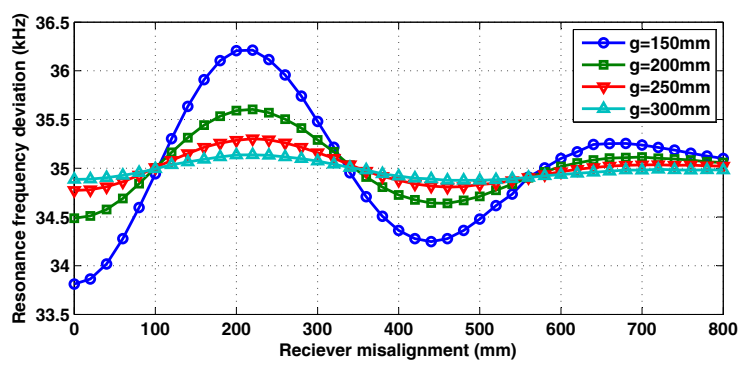

(b)

Figure 3.28: Experimental frequency measurement results: (a) Dynamic resonance frequency variations during horizontal movement of the secondary pad at $50 \mathrm{~mm} / \mathrm{s}$ speed and $200 \mathrm{~mm}$ air gap, (b) Resonance frequency measurements with variations in distance between the transmitter and receiver power pads (g: air gap).

different vertical and horizontal alignments and are presented in Fig. 3.28. Fig. 3.28(a) shows the resonance frequency of the IPT system as it dynamically changes when the secondary pad moves horizontally at $200 \mathrm{~mm}$ air gap and $50 \mathrm{~mm} / \mathrm{s}$ speed. This figure shows that the power controller effectively tracks the resonance frequency of the system in dynamically varying conditions. Also, Fig 15(b) shows the steadystate resonance frequency at different horizontal and vertical displacements which is captured experimentally using the IPT system setup. The results also show that the resonance frequency of the system can deviate by $3.5 \%$ due to horizontal misalignments. It is important to note that in the presented alignment experiment, the vehicle chassis which may impact the characteristics of the IPT system is not considered. 


\subsection{Summary}

A self-tuning soft-switching controller for H-bridge converters for power transfer regulation of IPT systems has been introduced. The self-tuning capability of the proposed controller allows synchronization of the switching operations with the resonance current of the IPT system and enables soft-switching operations to achieve a high efficiency. A simplified design for the proposed controller is introduced which eliminates the need for high-priced DSP/FPGA solutions and enables higher operating frequencies. The simulation results and experimental studies on a case study IPT system show that the proposed controller effectively regulates the resonant current around a desired value, synchronizes the switching operations with the resonant current and enables soft-switching operations. Although the proposed controller is designed for two-stage $\mathrm{AC} / \mathrm{DC} / \mathrm{AC}$ converter topologies, using the same design methodology it can be designed for any type of converter topology.

Furthermore, a controller that can be used in inductive EV charging systems to achieve multiple charging levels is introduced. The proposed controller has 10 userdefined charging levels and is capable of self-tuning the switching operations of the converter to the resonance frequency of the IPT system, and therefore eliminates the need for switching frequency tuning. Also, it enables soft-switching operations (ZCS) in the converter, which will result in a significant increase in the efficiency of the power electronic converter. Furthermore, the implementation of the proposed controller based on a simplified circuit eliminates the need for DSP/FPGA based solutions and enables high operating frequencies which are usually required in IPT systems. The experimental test results on the IPT test-bed conform with the simulation results and verify the effectiveness of the proposed controller at different charging levels. 


\section{CHAPTER 4}

\section{SINGLE-PHASE SOFT-SWITCHED AC-AC MATRIX CONVERTERS WITH POWER CONTROLLER FOR BIDIRECTIONAL INDUCTIVE POWER TRANSFER SYSTEMS}

In this chapter, direct single-phase ac-ac matrix converters with self-tuning capability and soft-switching operations for bidirectional inductive power transfer (IPT) systems are introduced. The self-tuning capability of the converters makes them suitable for inductive electric vehicle charging applications where the system has variable operating conditions. The soft-switching operations of the converters significantly improve the efficiency and reduce the switching stress which in turn reduces the complexity of the switching commutation. Also, due to the elimination of DC conversion stage and short life electrolytic capacitors, it is expected to be more reliable and durable. A discrete-time sliding mode control (SMC) is proposed to regulate the power transfer level and its direction in the IPT system. Theoretical analysis and design methodology of the proposed SMC are presented in detail. The simulation analysis on a case study IPT system is carried out and the results show that the proposed converter with its SMC can fully regulate the output resonant current around a user-defined reference value. The proposed converter is specifically suitable for grid-to-vehicle (G2V) and vehicle-to-grid (V2G) connections through inductive electric vehicle $(\mathrm{EV})$ charging systems in residential sectors. The work presented in this chapter is published in $[183,184]$. 


\subsection{Self-Tuned Single-Phase AC-AC Converter for Bidirec- tional Inductive Power Transfer Systems}

In this section, a soft-switched single-phase single-stage AC-AC matrix converter with self-tuning capability for bidirectional IPT systems is presented. A sliding mode controller is designed to control the power transfer level and its direction. A simplified digital design for the proposed SMC is presented. The controller constantly synchronizes the switching operations of the converter with the resonant current and, thereby achieves zero-current switching (ZCS). The proposed bidirectional AC-AC converter along with its controller is modeled in MATLAB/Simulink and its effectiveness in IPT based G2V and V2G connections is analyzed and the results are presented.

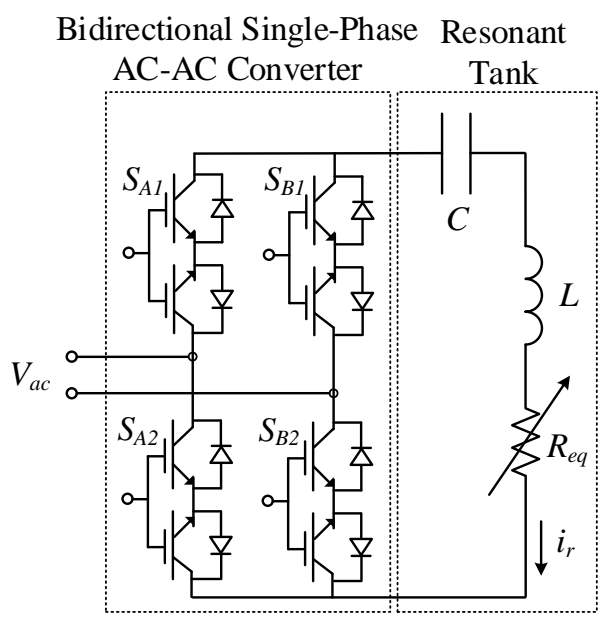

Figure 4.1: Single-phase ac-ac matrix converter with the simplified self-tuning controller. 


\subsubsection{Sliding Mode Controller Design for Single-Phase AC-}

\section{AC Converter}

In Fig. 4.1, a direct single-phase AC-AC converter comprised of four bidirectional switches $\left(S_{A 1}, S_{A 2}, S_{B 1}, S_{B 2}\right)$ are shown. The system is represented by an equivalent RLC circuit with a series capacitance $C$, a primary inductance $L$, and an equivalent resistance $R$ which characterizes the reflected impedance of the secondary circuit to the primary. In forward power transfer mode, the equivalent resistance has a positive value and in reverse power transfer mode, the equivalent resistance has a negative value. In order to design a sliding mode control (SMC) for the converter, the SMC laws should be obtained using theoretical analysis of the system. In order to design an SMC that enables soft-switching operations, it is assumed that the switching operations always occur at the resonant current zero-crossing points. Moreover, this assumption guarantees the resonance frequency self-tuning capability of the system. The design methodology is presented in the following section.

\subsubsection{Sliding Mode Control Law Derivation}

The state-space equations of an IPT system connected to the converter which is shown in Fig. 4.1 can be written as follows:

$$
\begin{gathered}
x(t)=\left[\begin{array}{c}
v_{c}(t) \\
i_{l}(t)
\end{array}\right] \\
\dot{x}(t)=A x(t)+B u(t) \\
y(t)=C x(t)+D u(t)
\end{gathered}
$$


where $v_{c}(t)$ is the voltage of the capacitor $C$ and $i_{l}(t)$ is the resonant current of the LC tank and:

$$
A=\left[\begin{array}{cc}
0 & \frac{1}{C} \\
-\frac{1}{L} & -\frac{R}{L}
\end{array}\right], B=\left[\begin{array}{c}
0 \\
\frac{1}{L}
\end{array}\right], C=\left[\begin{array}{ll}
0 & 1
\end{array}\right], D=0
$$

Assuming that the each half-cycle starts at a current zero-crossing, the initial condition and the input can be written as follows:

$$
x(0)=\left[\begin{array}{l}
v_{0} \\
0
\end{array}\right], \quad u=v_{t}
$$

where $v_{0}$ is the initial capacitor voltage and $v_{t}$ is the voltage across the LC tank. The solution to (4.2) in Laplace domain can be written as:

$$
X(s)=(s I-A)^{-1}\left[x(0)+B \frac{v_{t}}{s}\right]
$$

Using (4.3) and (4.4), (4.5) can be rewritten as follows:

$$
X(s)=\left[\begin{array}{c}
\frac{v_{t}}{s\left(C L s^{2}+C R s+1\right)}+\frac{C v_{0}(R+L s)}{C L s^{2}+C R s+1} \\
\frac{C v_{t}}{C L s^{2}+C R s+1}-\frac{C v_{0}}{C L s^{2}+C R s+1}
\end{array}\right]
$$

By applying inverse Laplace transform to (4.6) the time domain solution $x(t)$ can be written as follows:

$$
x(t)=\left[\begin{array}{c}
v_{t}+\left(v_{0}-v_{t}\right) e^{-t / \tau}\left[\cos (\omega t)+\frac{1}{\tau \omega} \sin (\omega t)\right] \\
\frac{\left(v_{t}-v_{0}\right)}{\omega L} e^{-t / \tau} \sin (\omega t)
\end{array}\right]
$$

where,

$$
\omega=\sqrt{\frac{1}{L C}-\frac{R^{2}}{4 L^{2}}}, \quad \tau=\frac{2 L}{R}
$$




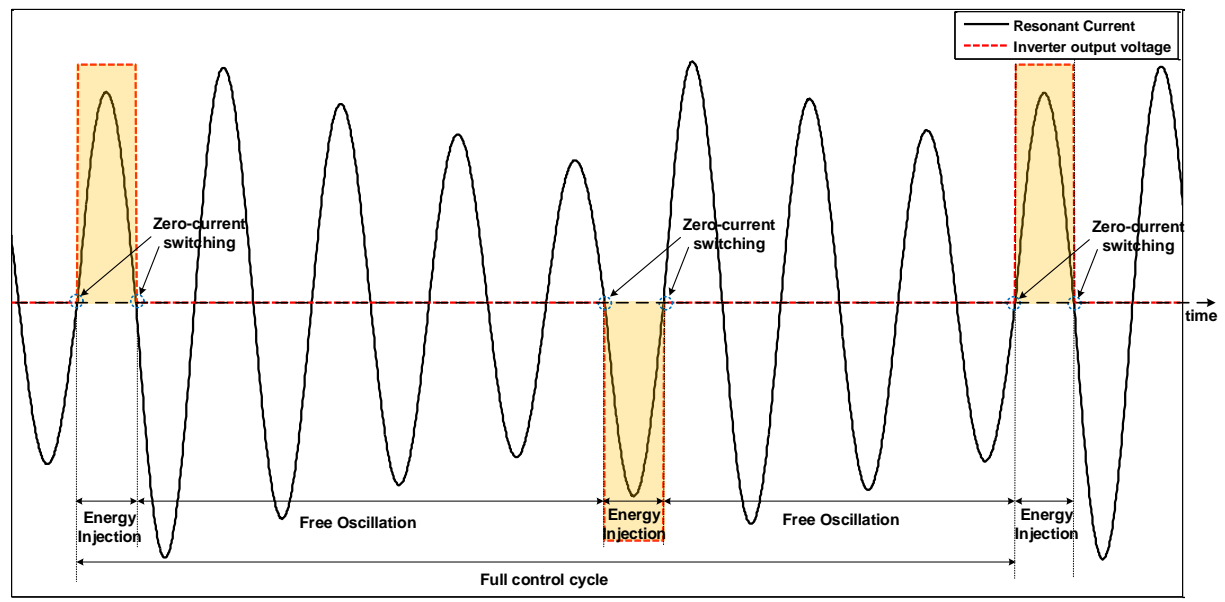

Figure 4.2: Conceptual plot of resonant current and inverter output voltage based on energy injection and free oscillation technique.

Equation (4.7) gives the resonant current and voltage for each half-cycle based on the initial capacitor voltage $v_{0}$ and the tank voltage $v_{t}$. The half-cycles wherein $v_{t}=V_{a c}$ or $v_{t}=-V_{a c}$ are energy-injection/regeneration half-cycles while the halfcycles wherein $v_{t}=0$ are free-oscillation half-cycles. Using (4.7), the peak resonant current in each half cycle $i_{p}$, which occurs at the time $t_{p}$ can be found by solving the following equation:

$$
\frac{d i_{l}}{d t}=\frac{\left(v_{t}-v_{0}\right)}{\omega L} e^{-t_{p} / \tau}\left[\omega \cos \left(\omega t_{p}\right)-\frac{1}{\tau} \sin \left(\omega t_{p}\right)\right]=0
$$

By simplifying (4.9), $t_{p}$ can be calculated as follows:

$$
t_{p}=\frac{\arctan (\tau \omega)}{\omega}
$$

By substituting (4.10) in (4.7), the peak resonant current $i_{p}$ can be calculated as the following equation:

$$
i_{p}=-\frac{\left(v_{t}-v_{0}\right)}{\omega L} e^{-\frac{\arctan (\tau \omega)}{\tau \omega}} \frac{\tau \omega}{\sqrt{1+(\tau \omega)^{2}}}
$$



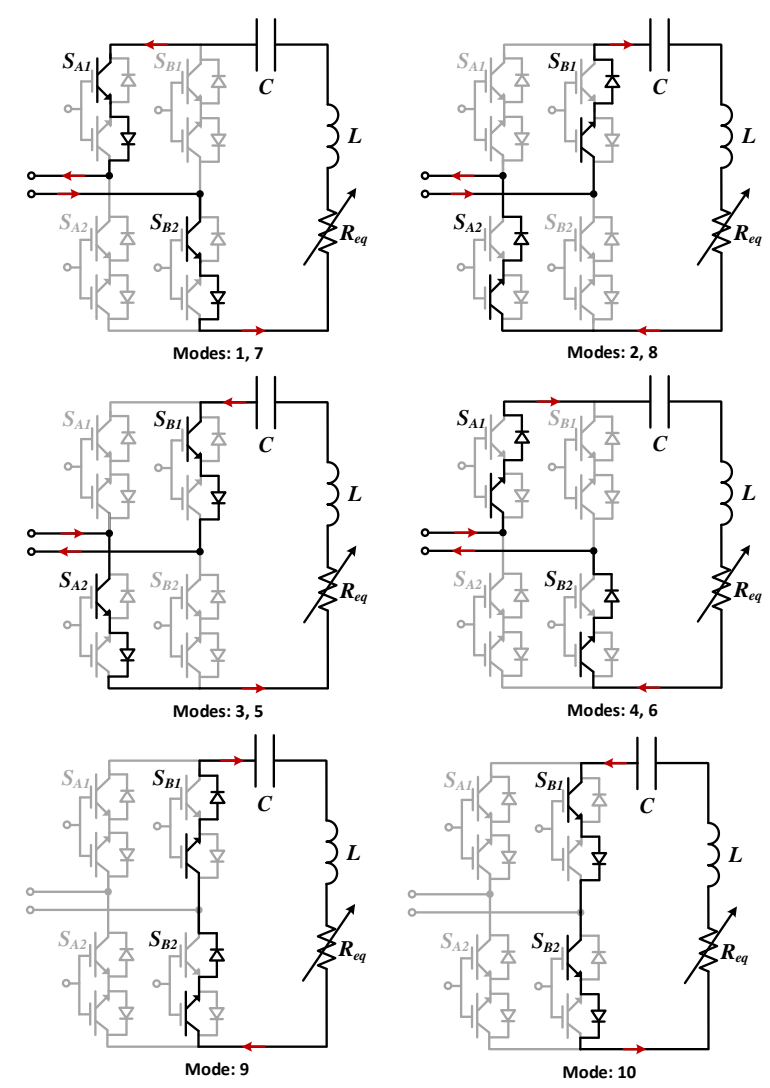

Figure 4.3: Resonant current path in 10 operation modes of the single-phase matrix converter.

In order to achieve zero-current switching operations, the transitions between different modes always occur at resonant current zero-crossing points. Therefore, the state-space model presented in (4.2) can be discretized in switching time points which results in a discrete-time SMC. Thus, the sampling time $T_{s}$ is defined as:

$$
T_{s}=\frac{\pi}{\omega}
$$

The equivalent discretized state-space model given by (4.2) can be rewritten as follows:

$$
x[k]=\left[\begin{array}{c}
v_{c}[k] \\
i_{l}[k]
\end{array}\right]
$$




$$
\begin{aligned}
& x[k+1]=A_{d} x[k]+B_{d} u[k] \\
& y[k+1]=C_{d} x[k]+D_{d} u[k]
\end{aligned}
$$

where $A_{d}$ and $B_{d}$ can be calculated as follows:

$$
\begin{gathered}
A_{d}=\mathcal{L}^{-1}\left\{(s I-A)^{-1}\right\}_{t=T_{s}} \\
B_{d}=A^{-1}\left(A_{d}-I\right) B
\end{gathered}
$$

Using (4.3), (4.15) and (4.16) can be simplified as follows:

$$
\begin{gathered}
A_{d}=\left[\begin{array}{cc}
-e^{-\pi / \tau \omega} & 0 \\
0 & -e^{-\pi / \tau \omega}
\end{array}\right] \\
B_{d}=\left[\begin{array}{cc}
1+e^{-\pi / \tau \omega} & 0
\end{array}\right]
\end{gathered}
$$

Also, based on the discretized model given by (4.14), the discretized peak resonant current can be rewritten as follows:

$$
i_{p}[k]=\left(u[k]-x_{1}[k]\right) \frac{\tau e^{-\arctan (\tau \omega) / \tau \omega}}{L \sqrt{1+(\tau \omega)^{2}}}
$$

Based on the peak resonant current given by (4.19), a sliding surface is defined as,

$$
\sigma[k]=\left|i_{p}[k]\right|-i_{r e f}
$$

where $\sigma[k]$ is the discrete sliding surface and $i_{r e f}$ is the reference current. The reaching law for the SMC can be formulated as follows [182]:

$$
(\sigma[k+1]-\sigma[k]) \sigma[k]<0
$$

Using (4.20) and (4.21) the following can be derived:

$$
\left(\left|i_{p}[k+1]\right|-\left|i_{p}[k]\right|\right) \sigma[k]>0
$$


Based on (4.22) the feedback control law $u[k]$ is picked so that the discrepancy between consecutive resonant current peaks and $\sigma[k]$ have same signs. In other words, whenever $\sigma[k]>0$ energy injection to the LC tank should be performed to increase the peak resonant current and whenever $\sigma[k]<0$ the LC tank should continue its free-oscillation. In the $\mathrm{AC}-\mathrm{AC}$ converter which is shown Fig. 4.1, the input voltage of the LC tank can be either $V_{a c},-V_{a c}$ or 0 . As a result, based on (3.4) the control law for the single-phase AC-AC converter can be derived as,

$$
u[k+1]= \begin{cases}\left|V_{a c}\right| & \sigma[k]>0, i_{p}[k]<0 \\ -\left|V_{a c}\right| & \sigma[k]>0, i_{p}[k]>0 \\ 0 & \sigma[k]<0\end{cases}
$$

Similarly, in reverse power transfer mode the following can be derived:

$$
u[k+1]= \begin{cases}-\left|V_{a c}\right| & \sigma[k]>0, i_{p}[k]<0 \\ \left|V_{a c}\right| & \sigma[k]>0, i_{p}[k]>0 \\ 0 & \sigma[k]<0\end{cases}
$$

Based on (4.23) and (4.24), the single-phase AC-AC converter will have 10 operation modes which are presented in Table 4.1. These modes are determined based on the direction of resonant current $\left(S_{c}\right)$, input voltage polarity $\left(S_{v}\right)$, reverse power flow signal $\left(S_{r}\right)$ and energy injection/regeneration signal $\left(S_{i}\right)$. In Fig. 4.3, the resonant current path in 10 operation modes are presented. According to Table 4.1 and Fig. 4.3, the switching signals of the AC-AC converter can be obtained as,

$$
\begin{aligned}
& S_{A 1}=\left(S_{c}^{*} S_{v}+\overline{S_{c}^{*} S_{v}}\right) S_{i}^{*}, \quad S_{A 2}=\left(\overline{S_{c}^{*}} S_{v}+S_{c}^{*} \overline{S_{v}}\right) S_{i}^{*} \\
& S_{B 1}=\left(S_{c}^{*} S_{v}+\overline{S_{c}^{*} S_{v}}\right) S_{i}^{*}+\overline{S_{i}^{*}}, \quad S_{B 2}=\left(\overline{S_{c}^{*}} S_{v}+S_{c}^{*} \overline{S_{v}}\right) S_{i}^{*}+\overline{S_{i}^{*}}
\end{aligned}
$$


Table 4.1: Operation modes of the single-phase AC-AC converter.

\begin{tabular}{l|llll|l}
\hline Mode & $\begin{array}{l}\text { Current } \\
\text { direction } \\
\left(S_{c}\right)\end{array}$ & $\begin{array}{l}\text { Supply volt- } \\
\text { age polarity } \\
\left(S_{v}\right)\end{array}$ & $\begin{array}{l}\text { Reverse } \\
\text { power } \\
\text { flow }\left(S_{r}\right)\end{array}$ & $\begin{array}{l}\text { Energy } \\
\text { injection- } \\
\text { regeneration } \\
\left(S_{i}^{*}\right)\end{array}$ & $\begin{array}{l}\text { ON } \\
\text { switches }\end{array}$ \\
\hline 1 & 0 & 0 & 0 & 1 & $S_{A 1}, S_{B 2}$ \\
2 & 1 & 0 & 0 & 1 & $S_{A 2}, S_{B 1}$ \\
3 & 0 & 1 & 0 & 1 & $S_{A 2}, S_{B 1}$ \\
4 & 1 & 1 & 0 & 1 & $S_{A 1}, S_{B 2}$ \\
5 & 0 & 0 & 1 & 1 & $S_{A 2}, S_{B 1}$ \\
6 & 1 & 0 & 1 & 1 & $S_{A 1}, S_{B 2}$ \\
7 & 0 & 1 & 1 & 1 & $S_{A 1}, S_{B 2}$ \\
8 & 1 & 1 & 1 & 1 & $S_{A 2}, S_{B 1}$ \\
9 & 1 & - & - & 0 & $S_{B 1}, S_{B 2}$ \\
10 & 0 & - & - & 0 & $S_{B 1}, S_{B 2}$ \\
\hline
\end{tabular}

where, $S_{c}^{*}=S_{c} \oplus S_{r}$ and $S_{i}^{*}=S_{i} \oplus S_{r}$. The direction of the power transfer can be reversed by changing the $S_{r}$ signal. Based on the control laws for the SMC and corresponding switching signals given in (4.25), a digital control circuit is designed which is shown in Fig. 4.4. This controller is composed of differential comparators and logic gates. A resonant current measurement $\left(i_{r}\right)$ and $\mathrm{AC}$ input voltage measurement $\left(V_{a c}\right)$ are used as feed-back and the reference current $\left(i_{\text {ref }}\right)$ and power transfer direction $\left(S_{r}\right)$ are defined as inputs.

In Fig. 4.2, conceptual plots of the energy injection/regeneration signal, resonant current in forward power transfer mode are shown. As it can be seen, the controller constantly switches between energy-injection/regeneration modes to regulate the amplitude of the resonant current. In energy-injection/regeneration modes energy is injected/regenerated into/from the LC tank from/to the singlephase AC supply, thus increasing/decreasing the resonant current. Conversely, in free-oscillation modes the LC tank continues its oscillation without any energy injection/regeneration from/to single-phase AC supply, thus decreasing/increasing the resonant current. 


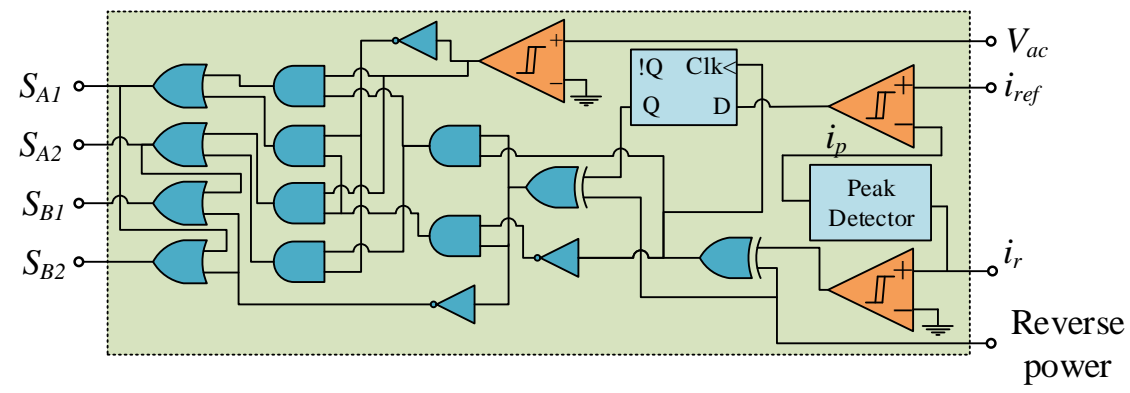

Figure 4.4: The simplified digital SMC based controller for the single-phase AC-AC converter.

\subsubsection{Simulation Results}

A bidirectional inductive battery charging system with the proposed single-phase $\mathrm{AC}-\mathrm{AC}$ converter as the primary converter is modeled in MATLAB/Simulink. The specifications of the case study IPT system is presented in Table 4.2. The simulations are carried out in forward and reverse power transfer modes (G2V and V2G) at Level 1 (L1: $3.3 \mathrm{~kW}$ ) and Level 2 (L2: $7.7 \mathrm{~kW}$ ) power levels (as defined in SAE J2954 standard [90]). Figure 4.5 shows the simulation results on the case study inductive battery charging system: (a) forward power transfer at 3.5kW power (Level 1), (b) forward power transfer at $8 \mathrm{~kW}$ power (Level 2), (c) reverse power transfer at $3.3 \mathrm{~kW}$ power (Level 1), (d) reverse power transfer at $7.5 \mathrm{~kW}$ power (Level 2). The results show that the single-phase AC-AC converter effectively enables bidirectional power transfer at desired power transfer levels. Figs. 4.5(a) and (b) show that in forward power transfer mode, the charging current has notches which correspond to the AC supply voltage zero-crossing points. This is due to the fact that in AC supply voltage zero-crossing points, although the controller forces the converter to operate at its maximum power transfer mode (without any free-oscillation modes), enough power is not being injected to the system to keep the charging current constant. Therefore, the battery charging current at secondary has such variations. 
Table 4.2: Specifications of the case study system.

\begin{tabular}{cc}
\hline Parameter & Value \\
\hline Self-inductances $\left(L_{p}, L_{s}\right)$ & $172 \mu \mathrm{H}$ \\
Compensation capacitors $\left(C_{p}, C_{s}\right)$ & $0.12 \mu \mathrm{F}$ \\
Resonance frequency $\left(f_{r}\right)$ & $35 \mathrm{kHz}$ \\
Grid voltage $\left(V_{a c}\right)$ & $120 \mathrm{~V}$ \\
Grid frequency $\left(f_{a c}\right)$ & $60 \mathrm{~Hz}$ \\
Battery voltage $\left(V_{b}\right)$ & $360 \mathrm{~V}$ \\
Battery capacity $\left(C_{b}\right)$ & $22 \mathrm{kWh}$ \\
\hline
\end{tabular}
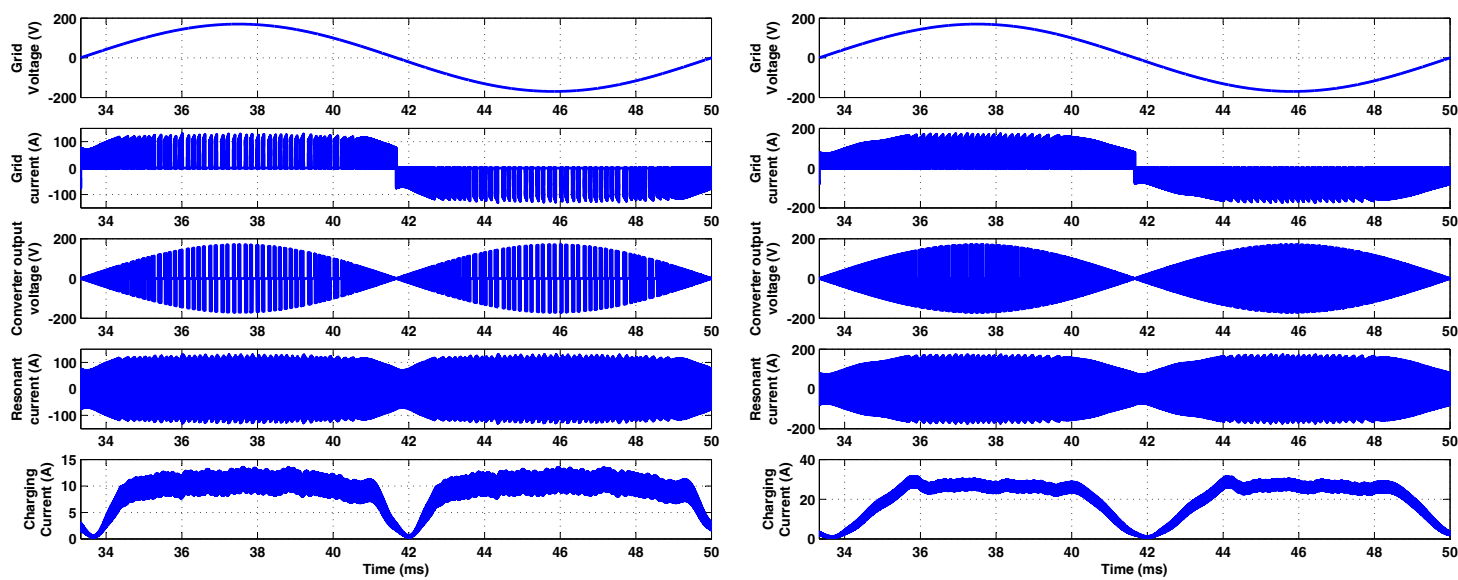

(a)

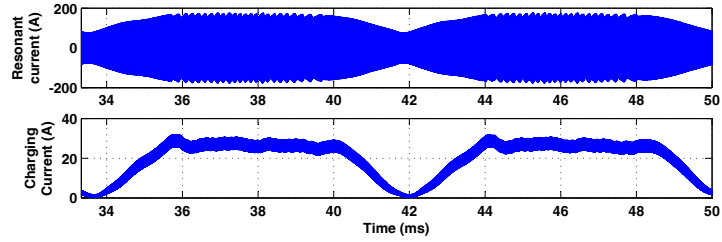

(b)
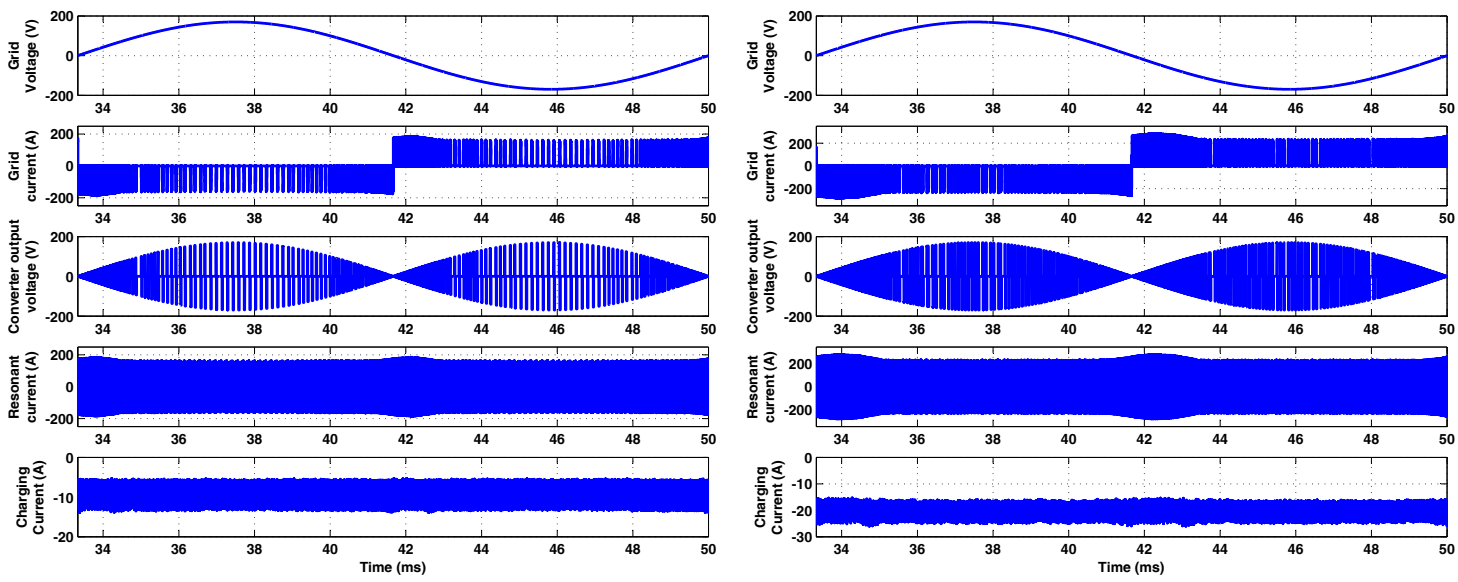

(c)

(d)

Figure 4.5: Simulation results on the case study inductive battery charging system: (a) forward power transfer at $3.5 \mathrm{~kW}$ power (Level 1), (b) forward power transfer at $8 \mathrm{~kW}$ power (Level 2), (c) reverse power transfer at $3.3 \mathrm{~kW}$ power (Level 1), (d) reverse power transfer at $7.5 \mathrm{~kW}$ power (Level 2). 


\subsection{Single-Phase Soft-Switched AC-AC Matrix Converter with Power Controller for Bidirectional Inductive Power Transfer Systems}

In this section, a direct soft-switched single-phase AC-AC matrix converters for bidirectional inductive power transfer (IPT) systems is proposed. Quantum energy injection/regeneration principle is used to design a simplified digital power controller that enables the converter to establish bidirectional power transfer between the IPT system and single-phase AC mains at a desired power level. The simplified controller can be implemented using basic logic circuit components, without the need for DSP/FPGA platforms, thereby reducing the complexity and the implementation cost. The converter benefits from resonance frequency tracking capability for the synchronization of switching operations of the converter with the resonant current which makes it ideal for dynamic IPT systems. Also, it benefits from soft-switching operations to achieve an enhanced efficiency and low electromagnetic interference (EMI). The converter is specifically suitable for establishing grid-to-vehicle (G2V) and vehicle-to-grid (V2G) connections through inductive electric vehicle charging/discharging systems. The proposed converter is analyzed theoretically, is simulated in MATLAB/Simulink, and finally is verified experimentally at low power on a case study IPT system. The results show that the proposed matrix converter can effectively establish bidirectional power transfer at different power levels with soft-switching operation and resonance frequency tracking capability.

With regular $120 \mathrm{~V} / 240 \mathrm{~V}$ single-phase supply, the power transfer levels that can be obtained include standard power levels as defined by SAE J2954 for lightduty EVs [90]. The power transfer regulation is achieved based on energy injec- 


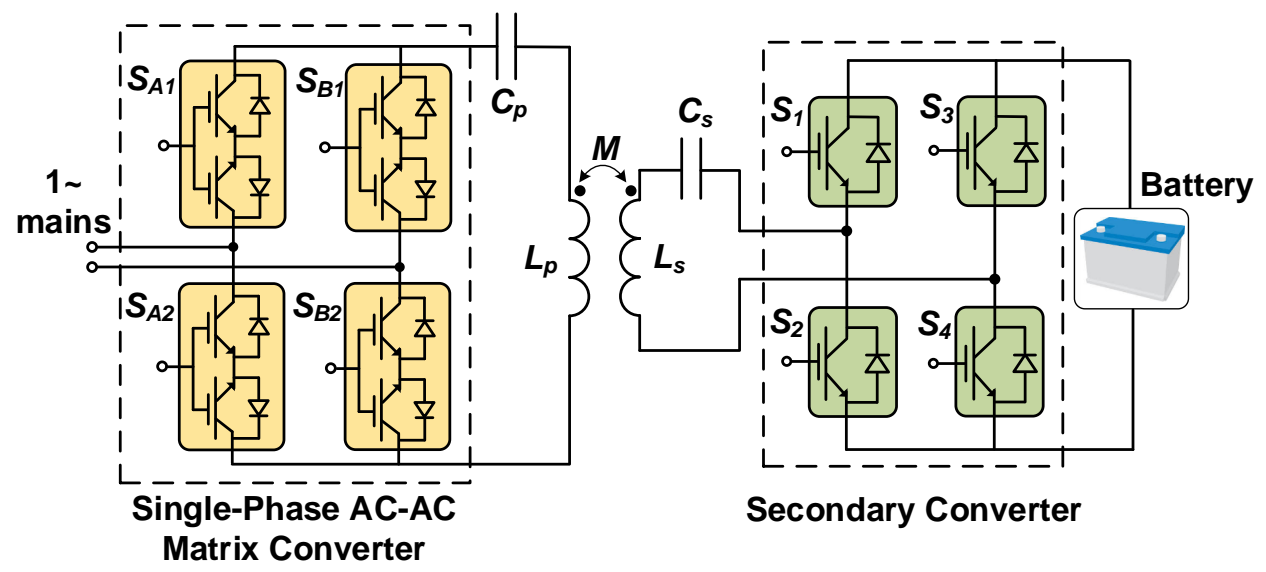

Figure 4.6: Series-compensated bidirectional IPT system with a single-phase AC$\mathrm{AC} \mathrm{MC}$ as the primary converter and a conventional H-bridge converter as the secondary converter.

tion/regeneration principle. The proposed controller benefits from resonance frequency tracking capability to constantly synchronize the switching operations of the converter with the resonant current. Also, it achieves zero-current switching (ZCS), which in turn eliminates the need for conventional multi-stage commutation techniques. The power controller is designed and implemented based on a digital circuit composed of basic logic level components without the use of FPGA/DSP solutions, thereby making it a low-cost solution. Theoretical and simulation analyses and experimental validations of the proposed direct $\mathrm{AC}-\mathrm{AC}$ matrix converter along with its controller are presented in detail.

\subsubsection{Power Transfer Controller for Direct Single-Phase AC-}

\section{AC Converter}

In Fig. 4.6, a series-series compensated bidirectional IPT system with a direct single-phase AC-AC MC as the primary converter and an H-bridge converter as the secondary converter is shown. The IPT system is represented by primary and sec- 


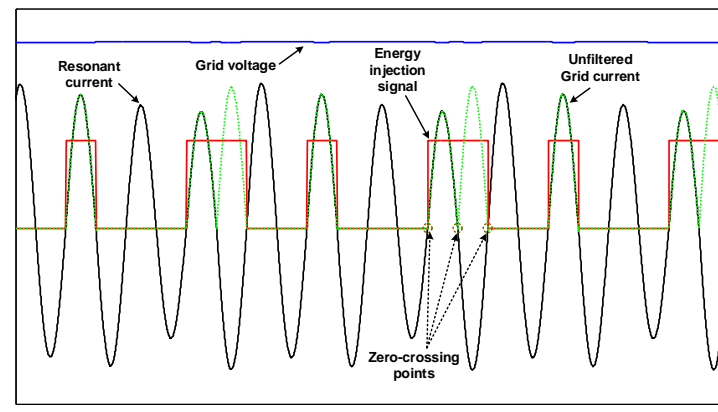

(a)

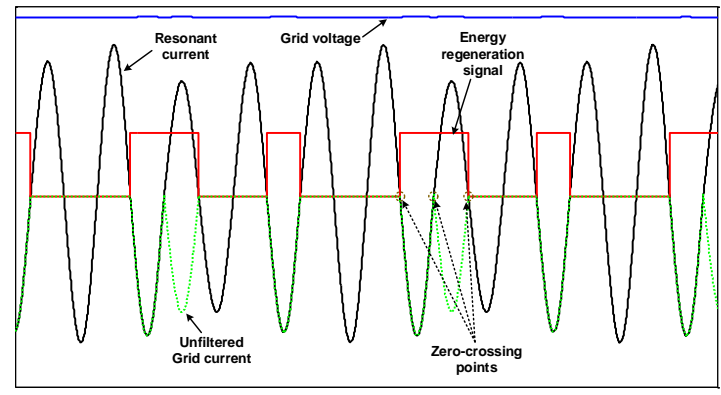

(c)

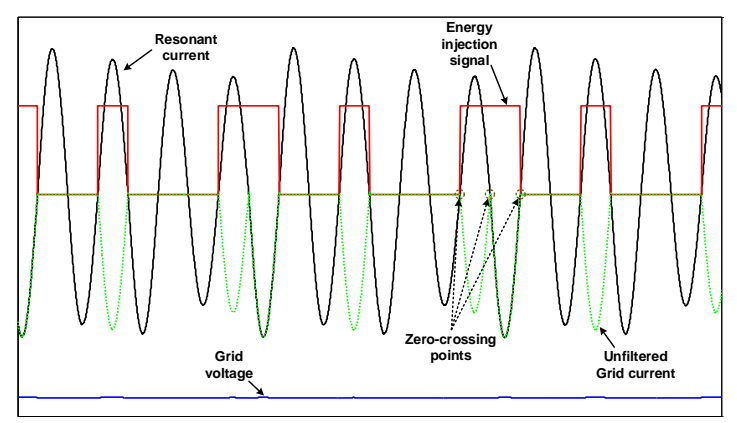

(b)

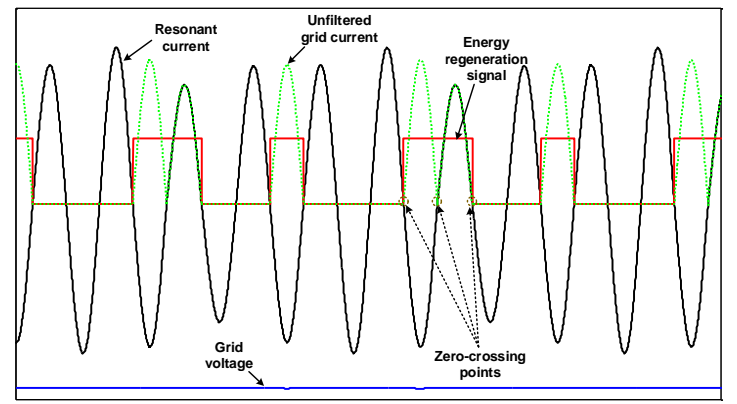

(d)

Figure 4.7: Concept plot of resonant current, energy injection or regeneration signal, grid current (unfiltered) and grid voltage: (a) forward power transfer mode with positive grid voltage, (b) forward power transfer mode with negative grid voltage, (c) reverse power transfer mode with positive grid voltage, (d) reverse power transfer mode with negative grid voltage.

ondary self-inductances $L_{p}$ and $L_{s}$, primary and secondary compensation capacitors $C_{p}$ and $C_{s}$. The primary converter is comprised of four bidirectional switches $\left(S_{A 1}\right.$, $\left.S_{A 2}, S_{B 1}, S_{B 2}\right)$.

A power controller is designed for the AC-AC converter that enables power transfer at the desired level by tuning the energy injection/regeneration rate in the IPT system. The controller takes resonant current $\left(i_{r}\right)$ and AC supply voltage $\left(v_{a c}\right)$ as feedbacks and generates four switching signals for the AC-AC converter. The controller is designed to enable soft-switching operations at the resonant current zero-crossing points. In other words, each operation mode starts and ends at current zero-crossing points, forming a half-cycle. The zero-current switching (ZCS) 

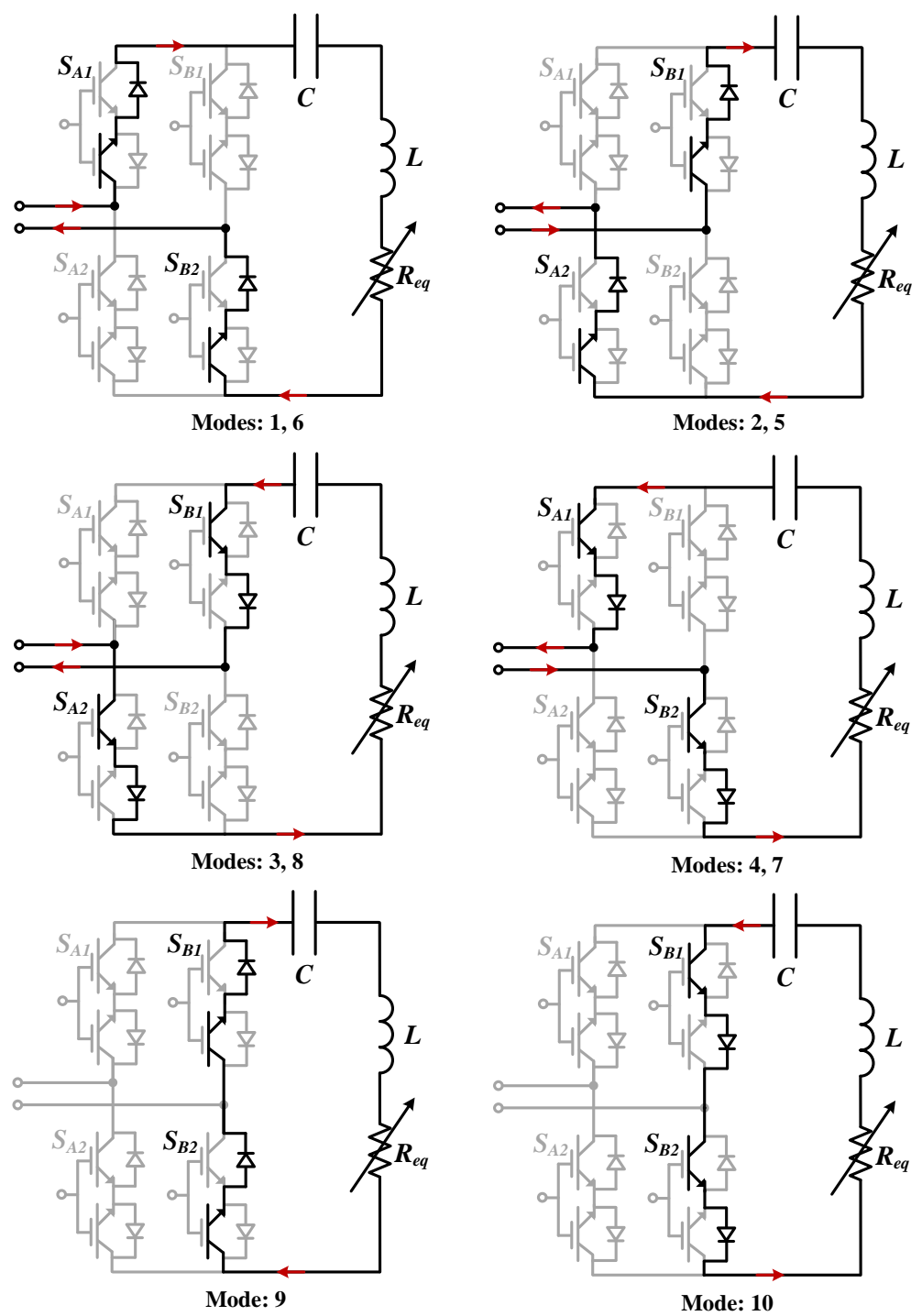

Figure 4.8: Resonant current path in the single-phase AC-AC converter in 10 operation modes.

significantly reduces the complexity of commutation usually required in matrix converters and eliminates the need for conventional four stage commutation techniques. The controller is composed of two main sections: switching logic and energy injection/regeneration frequency divider. The quantum energy injection/regeneration principle and the design methodology for each section of the controller are described as follows. 


\subsubsection{Quantum Energy Injection/Regeneration Principle for Power Transfer Control}

Loosely-coupled IPT systems operate based on resonant magnetic induction to enable power transmission through large air gaps. In such systems, the inductance of the coils and compensation capacitors form LC tanks (in both primary and secondary sides) in which a buffer energy is stored. The power transfer level in an IPT system can be regulated by controlling the buffer energy stored in the LC tanks. Quantum energy injection/regeneration to/from LC tank is a technique which can be used to control the buffer energy and consequently the power transfer level of the IPT system. This control method can be applied to the single-phase AC-AC matrix converter which is shown as a primary converter in Fig. 4.6. Based on this method energy can be transferred from/to the single-phase power source to/from the primary LC tank in the form of quantum energy pulses which exactly correspond to the resonant current half-cycles. In the half-cycles in which energy transfer is not desired, the IPT system enters a freewheeling mode in which the resonant current is freewheeled in order to continue its natural oscillation.

In Fig. 4.7, the application of this technique on the single-phase converter is conceptually shown at different operating conditions. This figure shows the concept plots of resonant current, energy injection or regeneration signal, grid current and grid voltage where (a), (b) represent forward power transfer mode with positive and negative grid voltages, and (c), (d) represent reverse power transfer mode with positive and negative grid voltages. As it can be seen, in forward power transfer mode, quantum energy injections increase the resonant current while in reverse power transfer mode, quantum energy regenerations decrease the resonant current. 
Table 4.3: Operation modes and corresponding switching states of the single-phase AC-AC matrix converter

\begin{tabular}{|c|c|c|c|c|c|c|c|c|c|}
\hline Mode & Type & $S_{r}\left(P_{g}<0\right)$ & $S_{c}\left(i_{r}>0\right)$ & $S_{v}\left(v_{a c}>0\right)$ & $S_{n r g}$ & $S_{A 1}$ & $S_{A 2}$ & $S_{B 1}$ & $S_{B 2}$ \\
\hline 1 & Energy injection & 0 & 1 & 1 & 1 & 1 & 0 & 0 & 1 \\
\hline 2 & Energy injection & 0 & 1 & 0 & 1 & 0 & 1 & 1 & 0 \\
\hline 3 & Energy injection & 0 & 0 & 1 & 1 & 0 & 1 & 1 & 0 \\
\hline 4 & Energy injection & 0 & 0 & 0 & 1 & 1 & 0 & 0 & 1 \\
\hline 5 & Energy regeneration & 1 & 1 & 1 & 1 & 0 & 1 & 1 & 0 \\
\hline 6 & Energy regeneration & 1 & 1 & 0 & 1 & 1 & 0 & 0 & 1 \\
\hline 7 & Energy regeneration & 1 & 0 & 1 & 1 & 1 & 0 & 0 & 1 \\
\hline 8 & Energy regeneration & 1 & 0 & 0 & 1 & 0 & 1 & 1 & 0 \\
\hline 9 & Free oscillation & - & 1 & - & 0 & 0 & 0 & 1 & 1 \\
\hline 10 & Free oscillation & - & 0 & - & 0 & 0 & 0 & 1 & 1 \\
\hline
\end{tabular}

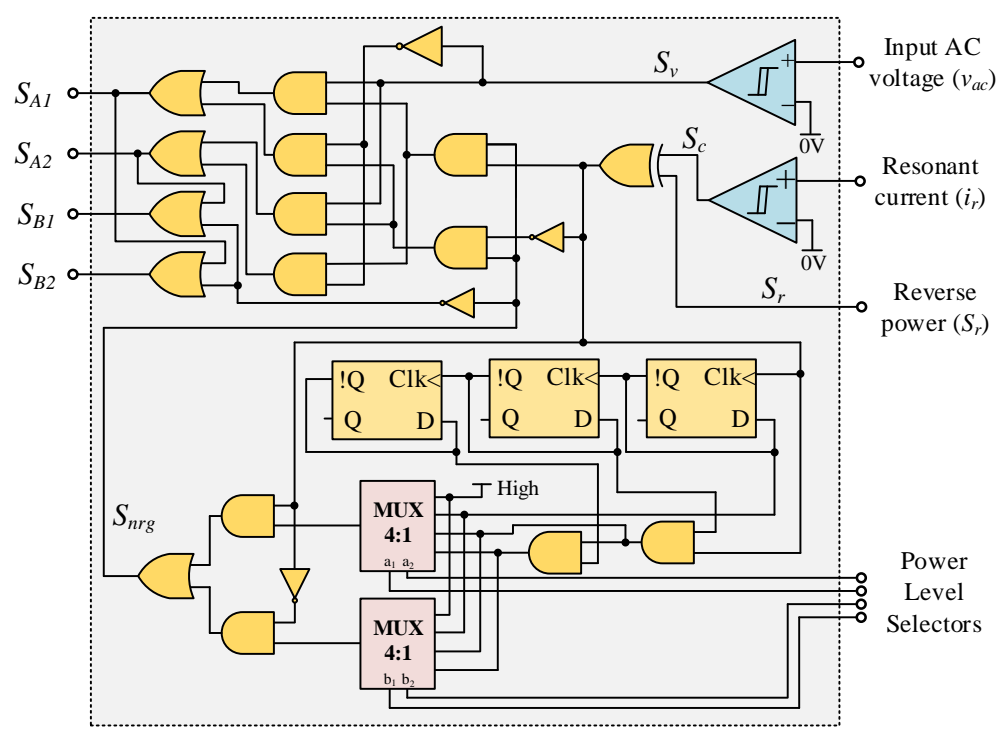

Figure 4.9: The proposed power controller for the primary single-phase AC-AC converter for bidirectional IPT systems.

\subsubsection{Switching Logic Design}

A switching logic based on the quantum energy injection/regeneration method for the single-phase AC-AC converter can be designed. This switching logic can be designed by defining different operation modes of the converter which are determined by the resonant current direction, grid voltage polarity, power transfer direction, and energy injection/regeneration signal. These operation modes are divided into three different types: energy-injection, energy-regeneration, and free-oscillation. A 


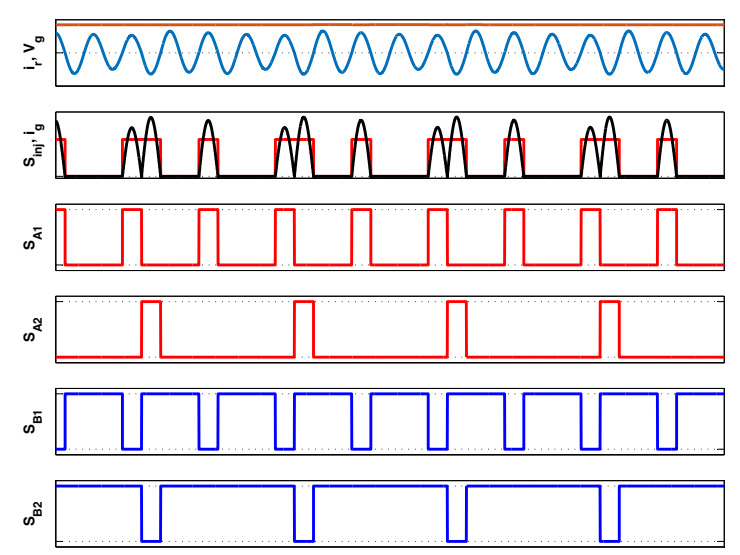

(a)

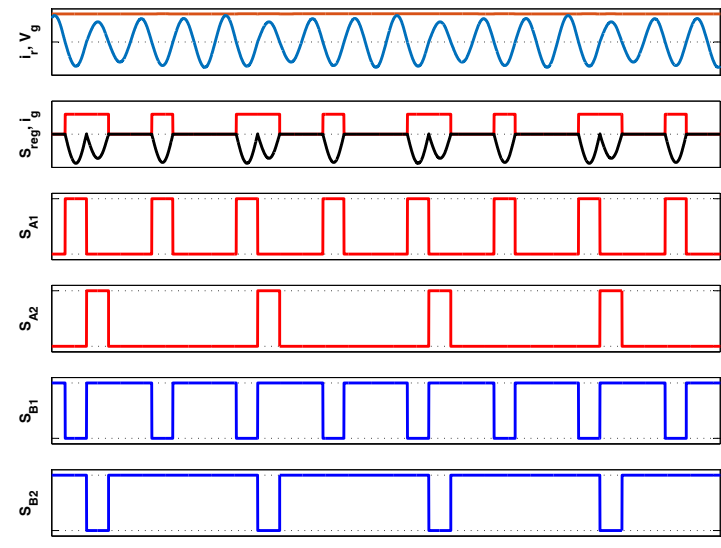

(c)

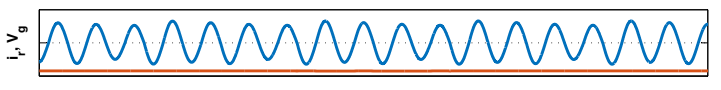
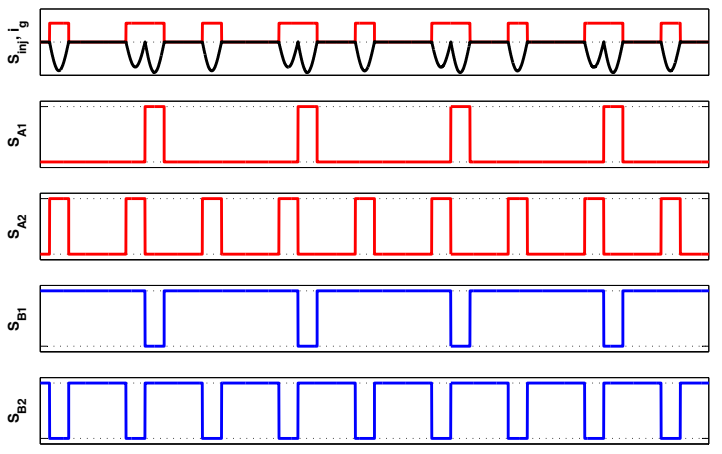

(b)
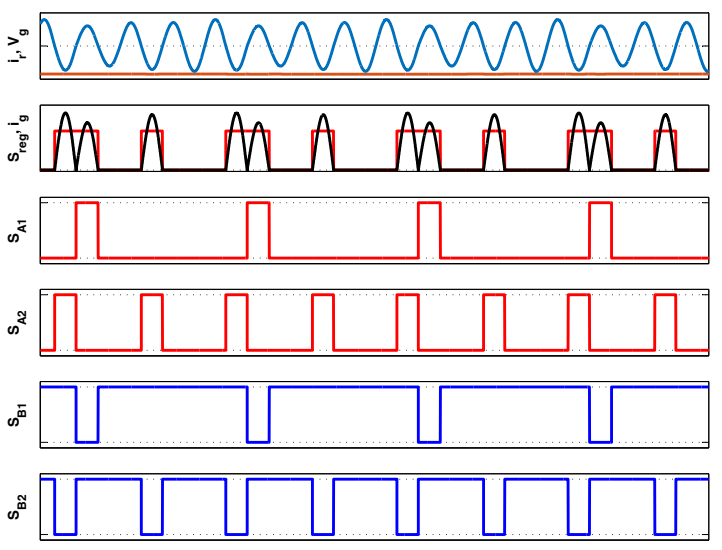

(d)

Figure 4.10: Concept plot of the switching signals of the converter $\left(S_{A 1}, S_{A 2}, S_{B 1}, S_{B 2}\right)$ along with the resonant current $\left(i_{r}\right)$, grid voltage $\left(V_{g}\right)$, energy injection/regeneration signal $\left(S_{i n j} / S_{r e g}\right)$, unfiltered grid current $\left(i_{g}\right)$ : (a) forward power transfer mode with positive grid voltage, (b) forward power transfer mode with negative grid voltage, (c) reverse power transfer mode with positive grid voltage, (d) reverse power transfer mode with negative grid voltage.

resonant current measurement $\left(i_{r}\right)$ and an input voltage measurement $\left(v_{a c}\right)$ are used as inputs for the switching logic. These modes are determined based on the direction of resonant current $\left(S_{c}: i_{r}>0\right)$, input voltage polarity $\left(S_{v}: v_{a c}>0\right)$, user-defined reverse power transfer signal $\left(S_{r}: P_{g}<0\right.$, where $P_{g}$ is the grid connection power), and energy flow signal (energy injection/regeneration signal) $\left(S_{n r g}\right)$. The $S_{n r g}$ signal is controlled by the second section of the controller which is described in Section 
4.2.1.3. Zero-cross detectors with tunable hysteresis bands are employed in order to find $S_{c}$ and $S_{v}$ using the resonant current and grid voltage input signals. The hysteresis band can be tuned to a desired value using a positive feedback from the source signals.

The switching logic is designed in a way that in energy injection modes the output voltage of the converter and the resonant current have the same sign, and in energy regeneration modes they have opposite signs. Also, in free oscillation modes, the output voltage of the converter is zero and the resonant current is freewheeled in the LC tank. For example in an energy injection mode $\left(S_{n r g}=1, S_{r}=0\right)$ with a positive resonant current $\left(i_{r}>0\right)$, a positive voltage should be applied to the IPT system and therefore, if $v_{a c}>0$ is positive, $S_{A 1}$ and $S_{B 2}$ are turned on, and if $v_{a c}<0$, $S_{A 2}$ and $S_{B 1}$ are turned on. In an energy regeneration mode $\left(S_{n r g}=1, S_{r}=1\right)$ with a negative resonant current $\left(i_{r}<0\right)$, a positive voltage should be applied to the IPT system and therefore, if $v_{a c}>0$ is positive, $S_{A 1}$ and $S_{B 2}$ are turned on, and if $v_{a c}<0, S_{A 2}$ and $S_{B 1}$ are turned on. According to these principles, 10 operation modes can be defined in the AC-AC converter, which are presented in Table 4.3 and the corresponding resonant current paths are shown in Fig. 4.8. Using Table 4.3, a switching logic can be easily obtained by expressing the switching signals $S_{A 1}, S_{A 2}$, $S_{B 1}$, and $S_{B 2}$ as boolean equations for each switching signal in terms of $S_{c}, S_{v}, S_{r}$, and $S_{n r g}$ as follows:

$$
\begin{aligned}
& S_{A 1}=S_{n r g} \overline{S_{r}}\left(S_{v} S_{c}+\overline{S_{v}} \overline{S_{c}}\right)+S_{n r g} S_{r}\left(\overline{S_{v}} S_{c}+S_{v} \overline{S_{c}}\right) \\
& S_{A 2}=S_{n r g} \overline{S_{r}}\left(\overline{S_{v}} S_{c}+S_{v} \overline{S_{c}}\right)+S_{n r g} S_{r}\left(S_{v} S_{c}+\overline{S_{v}} \overline{S_{c}}\right) \\
& S_{B 1}=S_{n r g} \overline{S_{r}}\left(\overline{S_{v}} S_{c}+S_{v} \overline{S_{c}}\right)+S_{n r g} S_{r}\left(S_{v} S_{c}+\overline{S_{v}} \overline{S_{c}}\right)+\overline{S_{n r g}} \\
& S_{B 2}=S_{n r g} \overline{S_{r}}\left(S_{v} S_{c}+\overline{S_{v}} \overline{S_{c}}\right)+S_{n r g} S_{r}\left(\overline{S_{v}} S_{c}+S_{v} \overline{S_{c}}\right)+\overline{S_{n r g}}
\end{aligned}
$$

The direction of the power flow can be reversed by changing the $S_{r}$ signal. The resonant current sign $S_{c}$ which is used as a clock source for the controller is switched 
at resonant current zero-crossing points, based on (4.26) the soft-switching operations of the converter is ensured. Based on (4.26), a switching logic circuit is designed which is presented in Fig. 4.9.

In order to achieve the soft-switching operations in the converter, the transitions between operation modes are performed at zero-crossing points. This is due to the fact that the resonant current $\operatorname{sign}\left(S_{c}: i_{r}>0\right)$ changes at each resonant current zero-crossing point. Also, the controller should be designed in such a way that any change in the energy injection/regeneration signal $\left(S_{n r g}\right)$ occurs at resonant current zero-crossing points. Thereby, according to the boolean equations given in (4.26), the converter achieves zero-current switching.

\subsubsection{Power Transfer Controller}

In an IPT system, the power transfer level can be controlled by regulating the energy transfer rate in the system. This can be achieved by controlling the number energy injection/regeneration pulses in a specific control cycle. In this study, a control cycle consisting of 8 resonant current cycles (16 half-cycles) is considered and the power transfer control is achieved by regulating the number of energy injection/regeneration half-cycles. The proposed power controller which is shown in Fig. 4.9, can change the number of energy injection/regeneration half-cycles to $1,2,4$, and 8 for both positive and negative half-cycles of the resonant current. This digital controller is designed using AND, OR, NOT, XOR logic gates, flipflops, and multiplexers. The controller generates an energy injection/regeneration signal $\left(S_{n r g}\right)$ based on the user-defined power transfer settings (selectors) for both positive and negative resonant current half-cycles. Using the proposed power controller, 10 power transfer levels can be achieved which are presented in Table 3.6. 
Table 4.4: Power transfer levels obtained using the power controller

\begin{tabular}{c|c|c|c|c}
\hline \hline \multirow{2}{*}{ Power Level No. } & Selector state & \multicolumn{2}{|c|}{ Number of energy injection/regeneration pulses } & Voltage transfer \\
& $a_{1} a_{2} b_{1} b_{2}$ & positive pulses $(m)$ & negative pulses $(n)$ & ratio $\left(G_{v}\right)$ \\
\hline \hline 1 & 0000 & 8 & 8 & 1.0000 \\
2 & 0001 & 8 & 4 & 0.8660 \\
3 & 0010 & 8 & 2 & 0.7906 \\
4 & 0011 & 8 & 1 & 0.7500 \\
5 & 0101 & 4 & 4 & 0.7071 \\
6 & 0110 & 4 & 2 & 0.6124 \\
7 & 0111 & 4 & 1 & 0.5590 \\
8 & 1010 & 2 & 2 & 0.5000 \\
9 & 1011 & 2 & 1 & 0.4330 \\
10 & 1111 & 1 & 1 & 0.3536 \\
\hline
\end{tabular}

Each power level corresponds to a certain number of positive and negative energy injection/regeneration half-cycles, $n$ and $m$, and selector state $\left(a_{1} a_{2} b_{1} b_{2}\right)$.

In Fig. 4.7, resonant current $\left(i_{r}\right)$, grid voltage $\left(V_{g}\right)$, grid current $\left(i_{g}\right)$, and energy injection/regeneration signal $\left(S_{i n j} / S_{r e g}\right)$ in forward and reverse power transfer modes at power transfer level 6 are shown conceptually. This figure shows that using the power controller, the resonant current is rectified and directed to the single-phase grid connection in both directions. Also, the switching diagram of the converter at different operating conditions is presented as Fig. 4.10. This figure corresponds to the operating conditions shown in Fig. 4.7 and it represents the switching signals of the converter $\left(S_{A 1}, S_{A 2}, S_{B 1}, S_{B 2}\right)$ along with the resonant current, grid voltage, grid current, and energy injection/regeneration signal.

\subsubsection{Forward Power Transfer}

The forward power transfer mode $\left(S_{r}=0\right)$ enables energy transfer from the primary single-phase AC source to the secondary DC source. In this case, the MC constantly switches between energy-injection and free-oscillation modes. In energy-injection modes, energy is transferred to the IPT system and as a result, the resonant current is increased. In free-oscillation modes, energy transfer to the IPT system is avoided 
and the resonant current is allowed to circulate in the system and the energy stored in the LC tank is used as a source for energy transfer to the IPT system. Since the energy stored in the system reduces, the resonant current decreases. Therefore, switching between these two types of modes enables power transfer regulation in forward power transfer mode. In Figs. 4.7(a), (b), the resonant current, energy injection signal, grid current and grid voltage are shown conceptually. It is clear that the resonant current increases in energy injection modes and it decreases in free-oscillation modes.

\subsubsection{Reverse Power Transfer}

The reverse power transfer mode $\left(S_{r}=1\right)$, is used to enable energy transfer to the primary single-phase AC source from the secondary DC source to the primary $\mathrm{AC}$ source. In this case, the secondary converter acts as an inverter to allow the secondary structure operate as a transmitter and in the primary MC, the energyregeneration and free-oscillation modes are engaged. In this case, $R_{e q}$ which represents the secondary load reflected to primary (shown in Fig. 4) is negative. This means that $R_{e q}$ generates power (rather than consuming power) and injects power to the primary LC tank. In energy-regeneration modes, the MC transfers energy back to the primary AC source and therefore the resonant current decreases. This is done by directing the resonant current to the $\mathrm{AC}$ source with a reverse polarity as shown in Fig. 4.7 and thereby, the energy stored in the resonant tank is injected into the grid which results in a reduction in the energy stored in the LC tank and the resonant current. Also, in free-oscillation modes, the primary current is allowed to circulate in the IPT system and thereby, energy regeneration is avoided. However, as a result of the energy transfer from the secondary to primary, in free-oscillation modes, the energy stored in the primary LC tank increases which in turn increases 
the resonant current. Power transfer regulation in reverse power transfer mode is enabled by continuously switching between these two types of modes.

\subsubsection{Theoretical Analysis}

In this section, the power transfer rate and efficiency of the converter at different power levels are calculated theoretically and details are presented.

\subsubsection{Calculation of Power Transfer}

The power transfer rate of the converter at different levels can be calculated by analyzing the voltage harmonics that are generated by the converter. Since the IPT system is tuned to operate at the resonance frequency, only the harmonic components of the converter output voltage that have the resonance frequency mainly involve in the power transfer. The proposed power control method is based on a full control cycle which is composed of 8 resonant current cycles. Thus, the fundamental harmonics component at the resonance frequency can be calculated as follows:

$$
V_{1}^{m n}=\frac{\omega}{8 \pi} \int_{0}^{8 \times \frac{2 \pi}{\omega}} v_{\text {out }} \sin (\omega t) d t
$$

where $v_{\text {out }}$ is the instantaneous output voltage of the converter, $\omega$ is the angular resonance frequency, and $V_{1}^{m n}$ is the main harmonic component of the output voltage at power level corresponding to $n$ and $m$ number of energy injection/regeneration pulses in positive and negative half-cycles respectively. Based on the principles of operation of the converter explained in Section 4.2.1, $v_{\text {out }}$ can be formulated as,

$$
v_{\text {out }}= \begin{cases} \pm\left|v_{a c}\right| & \frac{2 i \pi}{\omega}<t<\frac{(2 i+1) \pi}{\omega}, \frac{(2 j+1) \pi}{\omega}<t<\frac{2(j+1) \pi}{\omega} \\ & i=0, \ldots, m-1, \quad j=0, \ldots, n-1 \\ 0 & \text { otherwise }\end{cases}
$$


where $v_{a c}$ is the instantaneous voltage of the single-phase $\mathrm{AC}$ mains. The first section of $v_{\text {out }}$ corresponds to energy injection/regeneration modes and the second section corresponds to free-oscillation modes. The sign of the $v_{\text {out }}$ in energy injection/regeneration modes is determined based on the power transfer mode, direction of the resonant current and the polarity of the instantaneous grid voltage. By expanding (4.27) using (4.28), the following is obtained:

$$
V_{1}^{m n}=\frac{ \pm \omega}{8 \pi}\left(\sum_{i=0}^{m-1} \int_{\frac{2 i \pi}{\omega}}^{\frac{(2 i+1) \pi}{\omega}}\left|v_{a c}\right| \sin (\omega t) d t+\sum_{i=0}^{n-1} \int_{\frac{(2 i+1) \pi}{\omega}}^{\frac{2(i+1) \pi}{\omega}}\left|v_{a c}\right| \sin (\omega t) d t\right)
$$

Due to the fact that the resonance frequency is much higher than the power frequency, the variations of grid voltage in a control cycle can be neglected, and therefore (4.29) can be simplified as follows:

$$
V_{1}^{m n}=\frac{ \pm(m+n)\left|v_{a c}\right|}{4 \pi}
$$

It should be noted that the calculated harmonic are time variant and it is dependent on the single-phase AC mains voltage. At the resonance frequency the equivalent RLC tank is purely resistive and therefore, the fundamental harmonic of the resonant current can be calculated as,

$$
I_{1}^{m n}=\frac{V_{1}^{m n}}{R_{e q}}=\frac{ \pm(m+n)\left|v_{a c}\right|}{4 \pi R_{e q}}
$$

where $R_{e q}$ is the equivalent resistance reflected to the primary (Fig. 4.8). Using (4.31), the power transfer rate can be obtained as:

$$
P_{t}=\frac{1}{2} R_{e q}\left(I_{1}^{m n}\right)^{2}=\frac{(m+n)^{2} v_{a c}^{2}}{32 \pi^{2} R_{e q}}
$$

where $P_{t}$ is the instantaneous power transfer rate between single-phase AC mains and IPT system. Since $v_{a c}$ is sinusoidal, the average power transfer $P_{m}$ in a full grid voltage cycle can be calculated in terms of the RMS grid voltage $V_{A C}$ as follows:

$$
P_{m}=\frac{(m+n)^{2} V_{A C}^{2}}{32 \pi^{2} R_{e q}}
$$


According to (4.33), the power controller regulates the power transfer rate by changing the number of energy injection/regeneration pulses $(n$ and $m$ ). It should be noted that in reverse power transfer mode, $R_{e q}$ is negative and therefore, $P_{m}$ will be negative.

\subsubsection{Calculation of Voltage Transfer Ratio (VTR)}

The voltage transfer ratio of the converter is defined as follows:

$$
G_{v}=\frac{V_{\text {out }}}{V_{A C}}
$$

where $V_{\text {out }}$ is the RMS output voltage of the converter which can be calculated as,

$$
V_{\text {out }}=\sqrt{\frac{\int_{0}^{2 \pi / \omega_{0}} V_{\text {out }}^{2} d t}{2 \pi / \omega_{0}}}
$$

where $\omega_{0}$ is the angular frequency of grid voltage. It can be shown that using (4.28), (4.35) can be simplified as,

$$
V_{\text {out }}=\sqrt{\frac{\frac{m+n}{16} \int_{0}^{2 \pi / \omega_{0}} v_{a c}^{2} d t}{2 \pi / \omega_{0}}}
$$

Based on the definition of $V_{A C}$ and (4.36), the relation between $V_{\text {out }}$ and $V_{A C}$ is derived as,

$$
V_{\text {out }}=\frac{\sqrt{m+n}}{4} V_{A C}
$$

Using (4.34) and (4.37), the voltage transfer ratio (VTR) of the converter can be found as,

$$
G_{v}=\frac{V_{\text {out }}}{V_{A C}}=\frac{\sqrt{m+n}}{4}
$$

Equation (4.38) is used in order to directly calculate the voltage transfer ratio at different power transfer levels as presented in the last column of Table 4.4. 


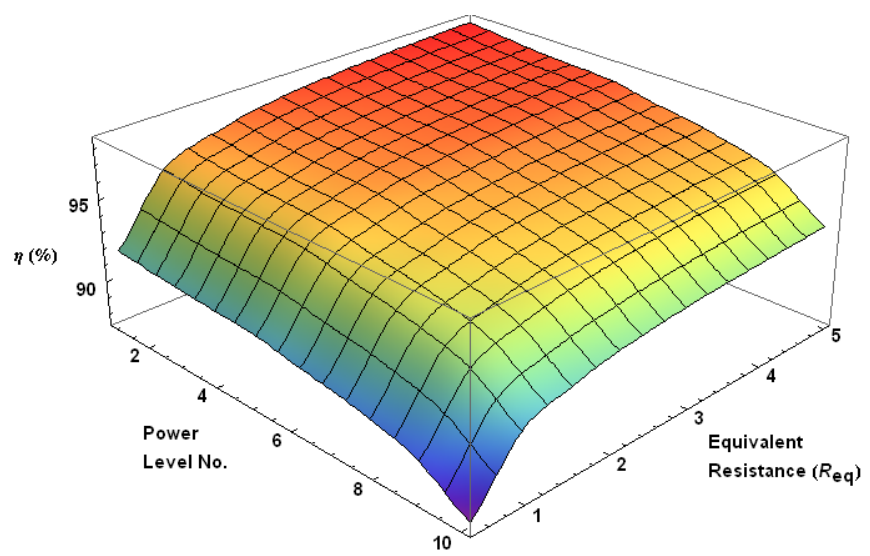

Figure 4.11: The efficiency of the single-phase AC-AC converter as a function of power transfer level and equivalent reflected resistance.

\subsubsection{Efficiency Analysis}

Since the converter achieves soft-switching operations, the switching losses are negligible and its conduction losses form the major power losses of the converter. Using the proposed power controller, the switching state of the converter corresponds to one of the operation modes presented in Table 4.3. In Fig. 4.8, it can be seen that in any operation mode, there are always two conducting bidirectional switches. Considering the fact that each conducting bidirectional switch is composed of one conducting power switch (MOSFET or IGBT) and one conducting body diode, the converter loss can be written as follows:

$$
\begin{aligned}
P_{\text {loss }}=2\left(P_{S}+P_{D}\right) & =2\left(V_{F S} I_{r m s}+R_{F} I_{r m s}^{2}+V_{F D} I_{r m s}\right) \\
& =2\left(V_{F S}+V_{F D}+R_{F} I_{r m s}\right) I_{r m s}
\end{aligned}
$$

Using (4.32) and (4.39), the efficiency of the converter can be obtained as,

$$
\eta=\frac{P_{t}}{P_{t}+P_{\text {loss }}}=\frac{1}{1+\frac{R_{F}}{R_{e q}}+\frac{4 \pi\left(V_{F S}+V_{F D}\right)}{(n+m) V_{r m s}}}
$$

The efficiency of a typical AC-AC converter as a function power level number and equivalent reflected resistance which is calculated analytically using (4.40), is 
presented in Fig. 4.11. This figure shows that as the power level decreases, the efficiency is decreased. Also, it can be seen that the converter can achieve up to $98 \%$ efficiency.

\subsubsection{Simulation Results}

A bidirectional inductive battery charging system with the proposed single-phase AC-AC MC converter as the primary converter and a conventional H-bridge converter as the secondary converter (Fig. 4.6) is modeled in MATLAB/Simulink. The primary converter is controlled using the proposed power controller and the secondary converter is switched using a self-tuning controller with battery charging/discharging current controller. The specifications of the case study system are presented in Table 4.5. The simulations were carried out in 10 power transfer levels in both forward and reverse power transfer modes with $120 \mathrm{~V}$ or $240 \mathrm{~V}$ single-phase mains, and the results are shown in Table 4.6 and Table 4.7. These tables show that the proposed single-phase AC-AC converter provides a wide range of power transfer levels for both G2V and V2G connections and the IPT system can achieve power conversion efficiencies as high as $98 \%$. In Fig. 4.12, the grid voltage and current, the energy injection/regeneration signals, the resonant current and the battery charging/discharging current for: (a) forward power transfer at level 2 with $120 \mathrm{~V}$ grid

Table 4.5: Specifications of the case study IPT system

\begin{tabular}{cc}
\hline \hline Parameter & Value \\
\hline Air gap & $200 \mathrm{~mm}$ \\
Self-inductances $\left(L_{p}, L_{s}\right)$ & $172 \mu \mathrm{H}$ \\
Compensation capacitors $\left(C_{p}, C_{s}\right)$ & $0.12 \mu \mathrm{F}$ \\
Resonance frequency $\left(f_{r}\right)$ & $35 \mathrm{kHz}$ \\
Grid voltage $\left(V_{A C}\right)$ & $120 \mathrm{~V}, 240 \mathrm{~V}$ \\
Grid frequency $\left(f_{a c}\right)$ & $60 \mathrm{~Hz}$ \\
Battery voltage $\left(V_{b}\right)$ & $360 \mathrm{~V}$ \\
Battery capacity $\left(C_{b}\right)$ & $22 \mathrm{kWh}$ \\
\hline
\end{tabular}



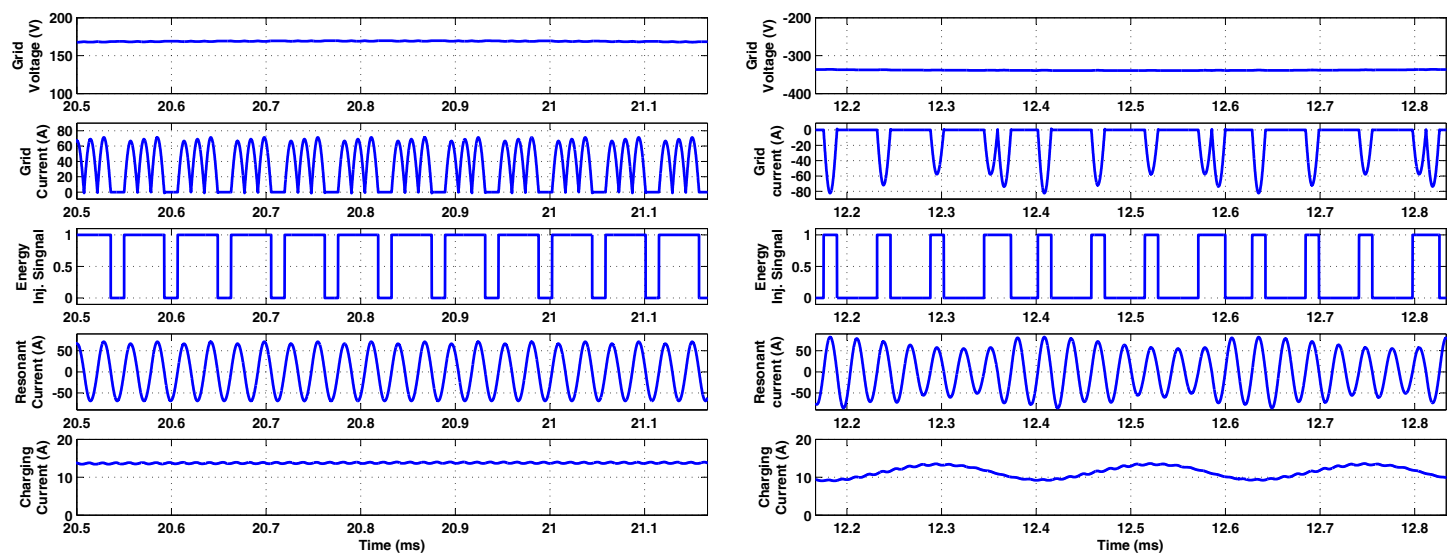

(a) forward power transfer at level 2 with (b) forward power transfer at level 6 with $120 \mathrm{~V}$ grid voltage at the positive peak of the $240 \mathrm{~V}$ grid voltage at the negative peak of grid voltage.

the grid voltage.
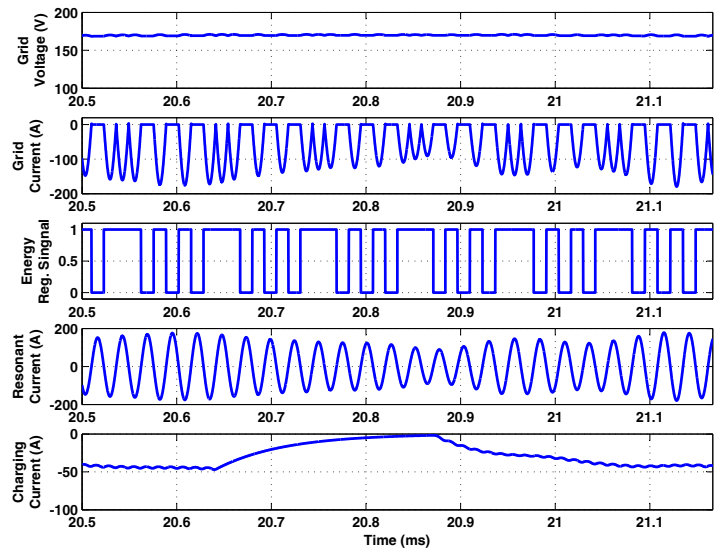

(c) reverse power transfer at level 3 with $120 \mathrm{~V}$ grid voltage at the positive peak of the grid voltage

Figure 4.12: Simulation results including the grid voltage and current, the energy injection/regeneration signals, the resonant current and the battery charging/discharging current.

voltage at the positive peak of the grid voltage, (b) forward power transfer at level 6 with $240 \mathrm{~V}$ grid voltage at the negative peak of the grid voltage, (c) reverse power transfer at level 3 with $120 \mathrm{~V}$ grid voltage at the positive peak of the grid voltage.

As it can be seen, since the resonance frequency of the IPT system is much higher than the frequency of the single-phase mains, the variations of the grid voltage in a 
Table 4.6: Simulation results of the case study IPT system in forward power transfer $(\mathrm{G} 2 \mathrm{~V})$ at different power levels

\begin{tabular}{|c|c|c|c|c|c|}
\hline $\begin{array}{l}\text { Power Transfer } \\
\text { Level }\end{array}$ & $\begin{array}{l}\text { Grid } \\
\text { voltage } \\
(\mathrm{V})\end{array}$ & $\begin{array}{l}\text { Grid } \\
\text { power } \\
(\mathrm{kW})\end{array}$ & $\begin{array}{l}\text { Grid } \\
\text { current } \\
\text { (A) }\end{array}$ & $\begin{array}{l}\text { Battery } \\
\text { current } \\
\text { (A) }\end{array}$ & $\begin{array}{l}\text { Battery } \\
\text { power } \\
(\mathrm{kW})\end{array}$ \\
\hline \multirow{2}{*}{1} & 120 & 4.77 & 48.52 & 13.18 & -4.61 \\
\hline & 240 & 15.52 & 74.16 & 46.8 & -15.09 \\
\hline \multirow{2}{*}{2} & 120 & 3.52 & 41.41 & 9.64 & -3.37 \\
\hline & 240 & 7.85 & 45.15 & 22.11 & -7.64 \\
\hline \multirow{2}{*}{3} & 120 & 2.92 & 37.58 & 7.94 & -2.77 \\
\hline & 240 & 6.15 & 39.30 & 17.11 & -5.97 \\
\hline \multirow{2}{*}{4} & 120 & 2.62 & 35.57 & 7.12 & -2.48 \\
\hline & 240 & 5.49 & 37.15 & 15.26 & -5.31 \\
\hline \multirow{2}{*}{5} & 120 & 2.32 & 33.37 & 6.26 & -2.18 \\
\hline & 240 & 4.78 & 34.37 & 13.20 & -4.62 \\
\hline \multirow{2}{*}{6} & 120 & 1.73 & 28.64 & 4.60 & -1.59 \\
\hline & 240 & 3.53 & 29.34 & 9.66 & -3.38 \\
\hline \multirow{2}{*}{7} & 120 & 1.43 & 25.93 & 3.79 & -1.30 \\
\hline & 240 & 2.93 & 26.70 & 8.04 & -2.78 \\
\hline \multirow{2}{*}{8} & 120 & 1.14 & 22.95 & 2.94 & -1.01 \\
\hline & 240 & 2.33 & 23.69 & 6.28 & -2.18 \\
\hline \multirow{2}{*}{9} & 120 & 0.84 & 19.55 & 2.13 & -0.72 \\
\hline & 240 & 1.73 & 20.23 & 4.67 & -1.59 \\
\hline \multirow{2}{*}{10} & 120 & 0.54 & 15.48 & 1.28 & -0.43 \\
\hline & 240 & 1.14 & 16.21 & 2.95 & -1.01 \\
\hline
\end{tabular}

few cycles of the resonant current is negligible and the grid voltage can be assumed to be constant. Figs. 4.12(a) and (b) show that in G2V connections, the grid current and voltage are of the same sign and the battery charging current is positive which confirm that the power that is being transferred to the battery is positive. Also, the figures show that free-oscillation modes cause a reduction in the energy stored in the IPT system and thereby reduce the resonant current. Conversely, Figs. 4.12(c) and (d) show that in V2G connections, the grid current and voltage have an opposite sign and the battery charging current is negative, which confirms that the power is being transferred to the grid is positive. Similarly, the figures show that free-oscillation modes cause an increase in the energy stored in the IPT system and thereby increase the resonant current. 
Table 4.7: Simulation results of the case study IPT system in reverse power transfer (V2G) at different power levels

\begin{tabular}{|c|c|c|c|c|c|}
\hline $\begin{array}{l}\text { Power Transfer } \\
\text { Level }\end{array}$ & $\begin{array}{l}\text { Grid } \\
\text { voltage } \\
\text { (V) }\end{array}$ & $\begin{array}{l}\text { Grid } \\
\text { power } \\
(\mathrm{kW})\end{array}$ & $\begin{array}{l}\text { Grid } \\
\text { current } \\
\text { (A) }\end{array}$ & $\begin{array}{l}\text { Battery } \\
\text { current } \\
\text { (A) }\end{array}$ & $\begin{array}{l}\text { Battery } \\
\text { power } \\
(\mathrm{kW})\end{array}$ \\
\hline \multirow{2}{*}{1} & 120 & -9.76 & 100.16 & 31.73 & 10.60 \\
\hline & 240 & -13.28 & 76.94 & 38.81 & 13.87 \\
\hline \multirow{2}{*}{2} & 120 & -7.19 & 85.45 & 26.08 & 8.01 \\
\hline & 240 & -10.95 & 73.10 & 33.13 & 11.60 \\
\hline \multirow{2}{*}{3} & 120 & -6.04 & 78.99 & 23.62 & 6.92 \\
\hline & 240 & -9.82 & 69.76 & 30.37 & 10.53 \\
\hline \multirow{2}{*}{4} & 120 & -5.44 & 74.81 & 22.30 & 6.30 \\
\hline & 240 & -9.16 & 68.08 & 29.24 & 9.85 \\
\hline \multirow{2}{*}{5} & 120 & -4.80 & 69.78 & 20.49 & 5.64 \\
\hline & 240 & -9.75 & 70.89 & 31.87 & 10.63 \\
\hline \multirow{2}{*}{6} & 120 & -3.64 & 61.46 & 17.77 & 4.46 \\
\hline & 240 & -7.16 & 60.78 & 26.01 & 8.00 \\
\hline \multirow{2}{*}{7} & 120 & -3.04 & 56.32 & 16.38 & 3.99 \\
\hline & 240 & -5.99 & 56.12 & 23.55 & 6.82 \\
\hline \multirow{2}{*}{8} & 120 & -2.47 & 50.89 & 15.35 & 3.49 \\
\hline & 240 & -4.79 & 49.64 & 20.47 & 5.63 \\
\hline \multirow{2}{*}{9} & 120 & -1.86 & 44.10 & 13.49 & 2.83 \\
\hline & 240 & -3.62 & 43.41 & 17.78 & 4.48 \\
\hline \multirow{2}{*}{10} & 120 & -1.19 & 34.77 & 10.27 & 2.08 \\
\hline & 240 & -2.43 & 35.56 & 14.87 & 3.42 \\
\hline
\end{tabular}

In Fig 4.13, the simulation results of a forward power transfer mode at the maximum power level $(15 \mathrm{~kW})$ in a full grid voltage cycle showing grid voltage, grid current, battery charging voltage and current are presented. This figure shows that the grid voltage variations lead to variations in battery charging current. Moreover, it can be seen that the LC filter has significantly reduced the harmonics associated with energy injection/regeneration current pulses of the primary side. Calculations show that the grid current has a Total Harmonic Distortion (THD) of $19 \%$. It should be noted that the grid current THD still can be improved more by using higher order power filters.

In Fig. 4.14, the efficiency calculations based on both the proposed power controller and a conventional controller at different power transfer levels are presented. The conventional power controller is simulated based on the firing angle control 

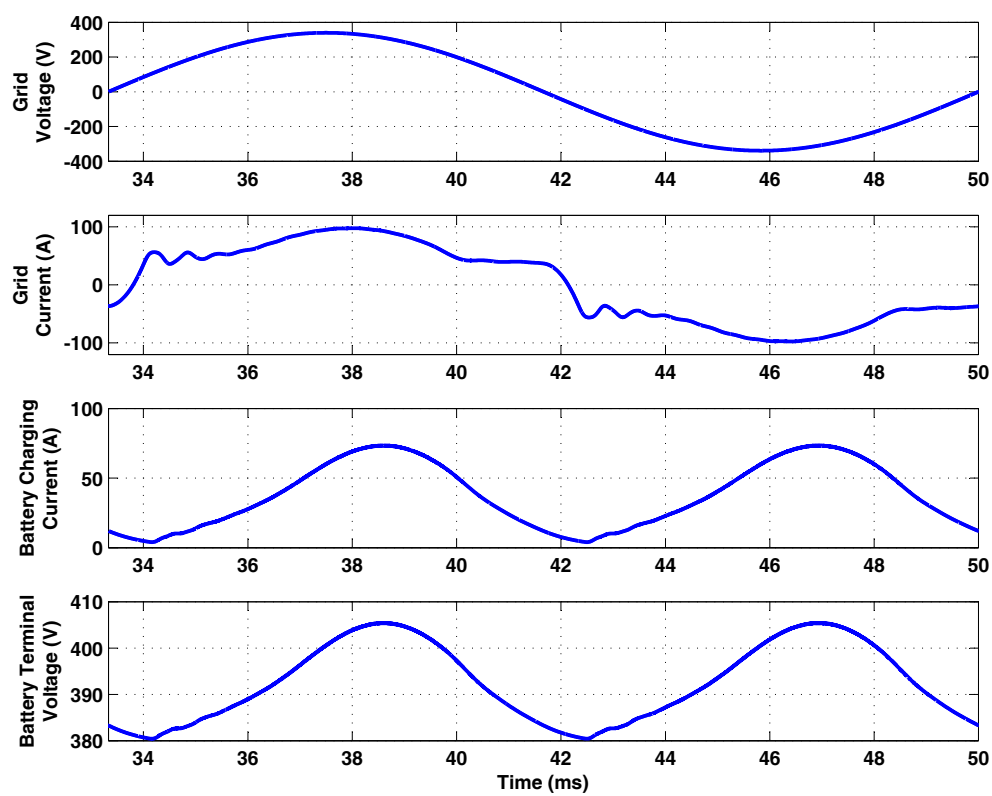

Figure 4.13: The simulation results of a G2V connection at the maximum power transfer level $(15 \mathrm{~kW})$ in a full grid voltage cycle showing grid voltage, grid current, battery charging voltage and current.

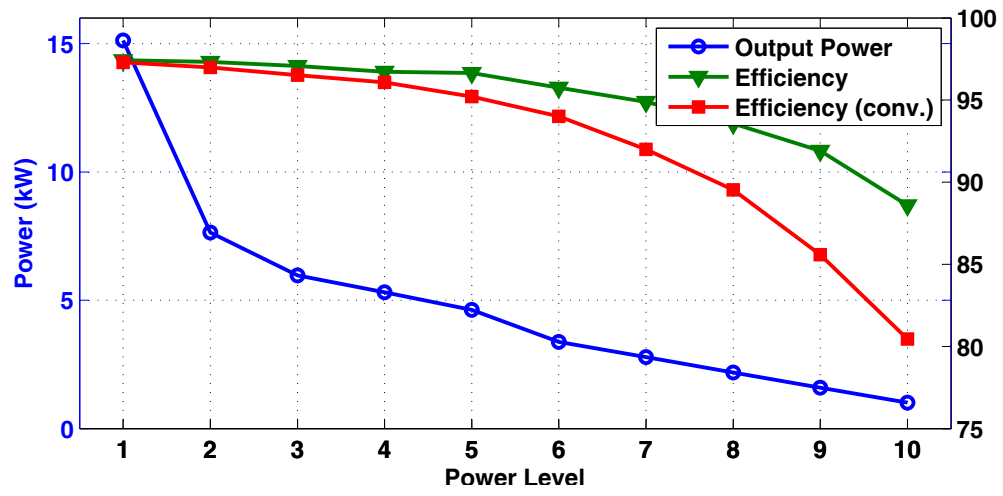

Figure 4.14: The efficiency of a single-phase AC-AC converter controlled using the proposed power controller and conventional controller at different power transfer levels calculated based on simulations.

method with respect to zero-current crossing points to regulate the power transfer rate. The results show that in both cases the converter can reach efficiencies as high as $98 \%$ at the maximum power level (Level 1). As the power level decreases, the proposed converter significantly outperforms the conventional controller and at 
Table 4.8: Specifications of the case study IPT system setup

\begin{tabular}{c|c|c}
\hline \hline Parameter & Components & Value \\
\hline Pad self-inductance & 15 turns, 10 AWG Litz wire & $172 \mu \mathrm{H}$ \\
Compensation capacitors & Film capacitor, FPG66Y0124J & $120 \mathrm{nF}$ \\
Primary supply & Variable three-phase AC supply & $20 \mathrm{~V}(\mathrm{LL}), 60 \mathrm{~Hz}$ \\
Secondary battery & Lead-acid battery & $12 \mathrm{~V}, 86.4 \mathrm{Wh}$ \\
\hline \hline
\end{tabular}

power level 10 it can achieve $8 \%$ higher efficiency. This is mainly due to the fact that the proposed power controller ensures soft-switching operations of the converter at all power transfer level.

\subsubsection{Experimental Results}

The proposed single-phase AC-AC converter and its power controller are built and experimental tests were carried out on a case study IPT system which is shown in Fig. 4.15. The IPT system setup includes series compensated transmitter and receiver power pads with 200mm air gap, primary AC-AC converter, secondary Hbridge converter. The power controller of the primary AC-AC converter is built according to the circuit presented in Fig. 4.9. A variable single-phase AC supply is used as a grid connection. The details of the IPT system setup specifications are presented in Table 4.8. An LC power filter is used on the primary side for miti-

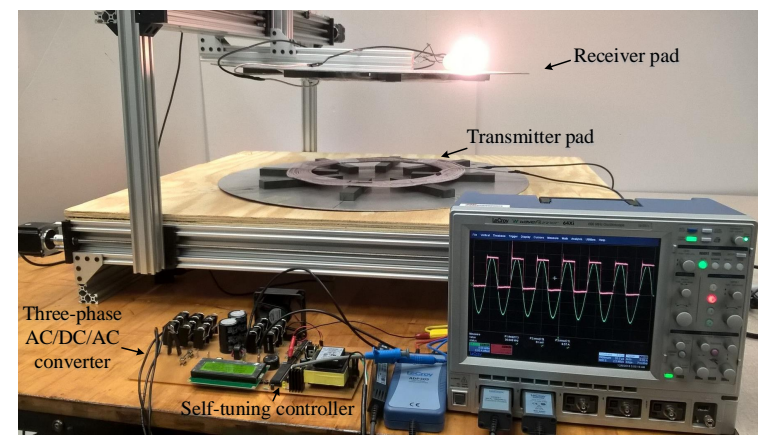

Figure 4.15: The IPT system setup comprised of primary and secondary pads, primary AC-AC matrix converter controlled using the proposed power transfer controller, and the secondary converter. 


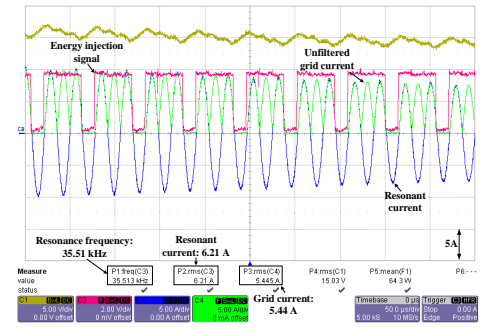

(a)

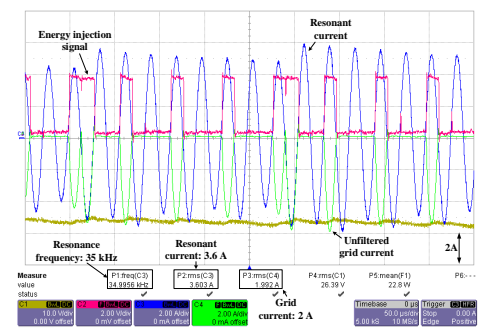

(d)

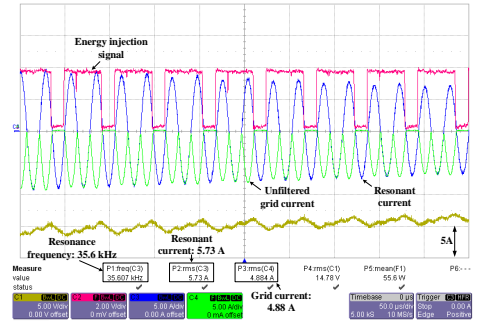

(b)

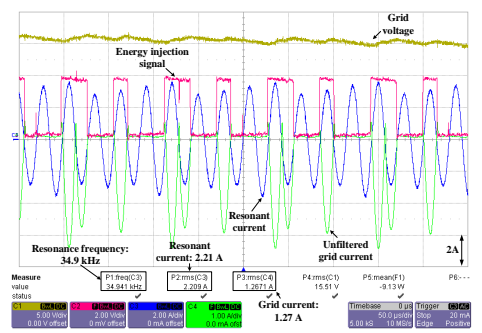

(e)

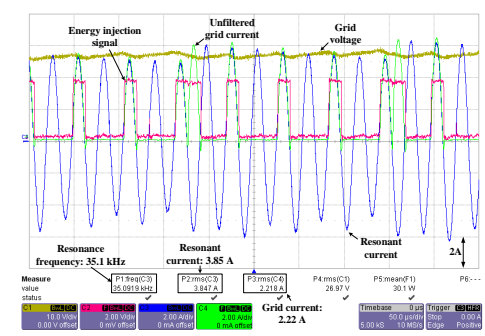

(c)

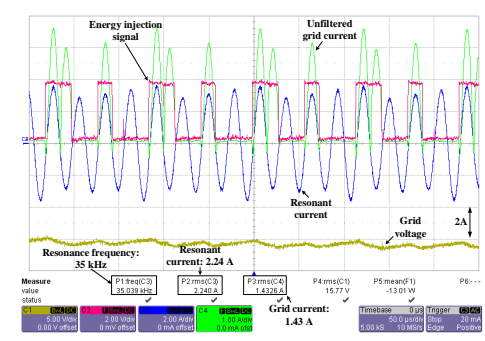

(f)

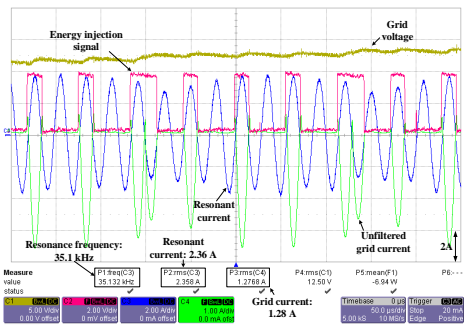

(g)

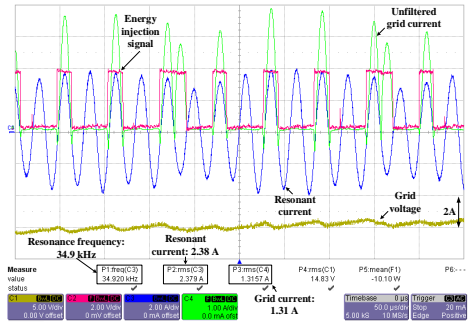

(h)

Figure 4.16: Experimental test results on the case study IPT system setup including the grid voltage and current (unfiltered), the energy injection/regeneration signals, and the resonant current: (a), (b) level 2 forward power transfer at positive and negative peak grid voltage, (c), (d) level 6 forward power transfer at positive and negative peak grid voltage, (e), (f) level 6 reverse power transfer at positive and negative peak grid voltage, (g), (h) level 7 reverse power transfer at positive and negative peak grid voltage.

gation of the harmonics generated by the proposed single-phase AC-AC converter. This filter is designed to have a cut-off frequency less than one-sixth of the switching frequency of the converter (resonance frequency). The LC filter is composed of $\mathrm{L}=40 \mu \mathrm{H}, \mathrm{C}=20 \mu \mathrm{F}$ with a cut-off frequency of $5.6 \mathrm{kHz}$. 
The experimental results including the grid voltage and current (unfiltered), the energy injection/regeneration signals, and the resonant current are shown in Fig. 4.16. Figures 4.16(a), (b), (c) and (d) show forward power transfer at levels 2 and 3 at positive and negative peak grid voltage. Figures 4.16(e), (f), (g) and (h) show reverse power transfer at levels 6 and 7 at positive and negative peak grid voltage. These figures show that the AC-AC converter can effectively establish single-phase $\mathrm{G} 2 \mathrm{~V}$ and $\mathrm{V} 2 \mathrm{G}$ connections at different power transfer levels with resonance frequency tracking capability and soft-switching operations.

As it is shown in Fig. 4.16, in forward power transfer modes, the grid current and voltage have same signs and therefore, the power is being transferred from the primary AC supply to the secondary. In reverse power transfer modes, the grid current and voltage have opposite signs and thus, the power is being transferred from the secondary to the primary AC supply. The power controller is designed to allow the resonant current to continue its damped oscillation regardless of power transfer level and direction of power. This enables the resonance frequency tracking capability and ensures high power transfer efficiency as the converter operates at the damped resonance frequency of the IPT system (frequency of resonant current). The transitions to different operation modes occur at the resonant current zero-crossing points which enable soft-switching operations. The grid voltage has small variations that are due to the energy injection/regeneration to/from the IPT system. The efficiency measurements at $95 \mathrm{~W}$ show that the single-phase AC-AC converter can achieve $92 \%$ efficiency at level 1 in both forward and reverse power transfer modes. The efficiency of the converter significantly increases as the power level increase and as it is shown in Fig. 4.14, it can achieve efficiencies as high as $98 \%$. 


\subsection{Summary}

The proposed self-tuned soft-switched single-phase AC-AC converter along with its SMC based controller can be used in IPT systems as an alternative for conventional two-stage converters. The reduced number of elements which is achieved by eliminating the DC conversion stage and corresponding bulky DC-link capacitors will lead to a compact and reliable converter. The proposed SMC based controller enables soft-switching operations which in turn can significantly enhance the efficiency of the converter. Since IPT systems usually have a variable operating condition, the self-tuning capability of the proposed converter is suitable for IPT systems. The controller is designed based on a simplified digital circuit that can achieve high operating frequencies without the need for DSP/FPGS solutions. Using the proposed controller the power transfer direction and level can be easily controlled. The bidirectional power transfer capability enables G2V and V2G connections through a single-phase power line, which can facilitate the grid integration of EVs through inductive charging links for residential sectors.

The proposed direct single-phase AC-AC converter along with the proposed power transfer controller can be used as an alternative to conventional two-stage converters for bidirectional IPT systems. Due to the elimination of AC-DC conver-

sion stage and bulky DC-link capacitors, the converter is more compact and reliable compared to conventional two-stage converters. The power transfer controller which is designed based on the energy injection/regeneration principle and is implemented based on simplified logic design, effectively enables bidirectional power transfer at 10 different power levels. The resonance frequency tracking capability ensures that the converter operates exactly at the damped resonance frequency of the IPT system and therefore, power transfer efficiency with high efficiency can be achieved. 
Also, the converter benefits from soft-switching operations that further enhances the efficiency of the converter. Finally, the structure of the single-phase matrix converter along with the simplified controller makes it suitable for residential inductive charging systems with bidirectional power flow capability. The resonance frequency tracking capability of the converter makes it suitable for inductive EV charging systems, in which the system may have variations. The bidirectional power transfer capability can be used to make single-phase G2V and V2G connections through IPT systems at multiple power levels including those defined by SAE J2954 standard. 


\section{CHAPTER 5}

\section{THREE-PHASE AC-AC MATRIX CONVERTER FOR INDUCTIVE POWER TRANSFER SYSTEM}

In this chapter the applications of Three-Phase AC-AC Matrix Converter for Inductive Power Transfer Systems are presented. Specifically, a direct three-phase ac-ac matrix converter for inductive power transfer (IPT) systems with soft-switching operation is introduced. The proposed topology is expected to have a high reliability and extended lifetime due to the soft-switching operation and elimination of short life electrolytic capacitors. The soft-switching operation will also reduce switching stress, switching loss and electromagnetic interference (EMI) of the converter. A variable frequency control strategy based on the energy injection and free oscillation technique is used to regulate the resonant current, the resonant voltage, and the output power. With the use of reverse blocking switches, the proposed converter can be built with a reduced number of switches (only seven), which will consequently increase the reliability, efficiency and reduce the cost of the converter. The converter operates in eight modes, which are described in detail. With the use of the proposed converter as the primary converter, simulation analysis and experimental implementations on a case study IPT system, show that the current regulation control method can fully regulate the output current and output power around user-defined reference values. Thus, makes it suitable for dynamic IPT applications, where the system has inherent variations. The work presented in this chapter is published in $[133,175,185]$. 


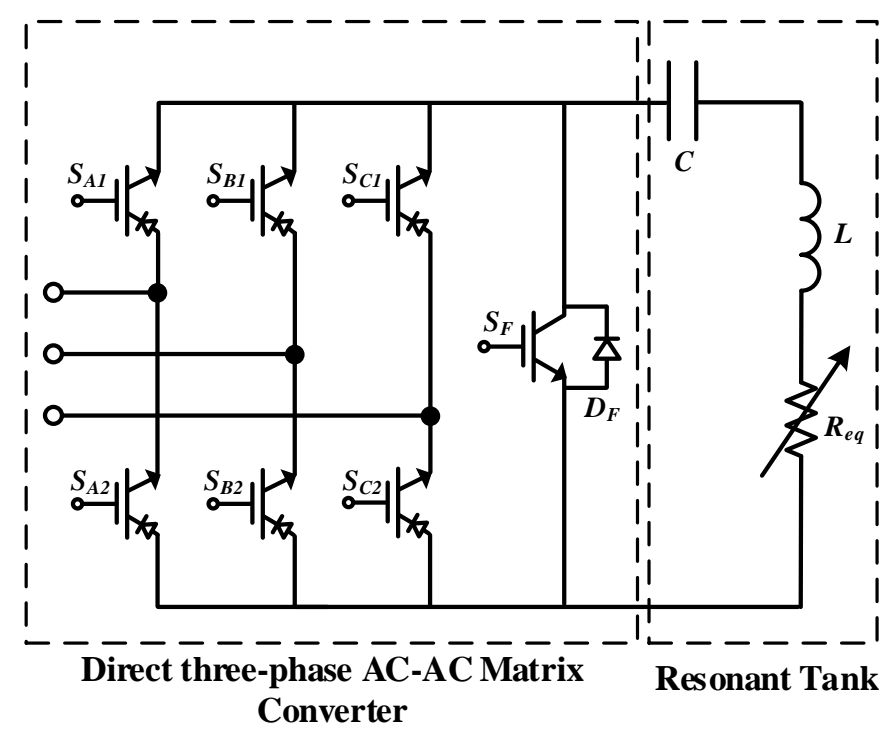

Figure 5.1: Proposed three-phase ac-ac matrix converter.

\subsection{Single-Stage Three-Phase AC-AC Matrix Converter for Inductive Power Transfer Systems}

In this chapter, a three-phase ac-ac matrix converter for IPT systems is proposed. The proposed matrix topology can be built using seven switches, which six of them are reverse blocking switches and one is a regular switch. A variable frequency control strategy based on the proposed converter is used, which is based on the energy injection and free oscillation technique. The key benefits of the proposed converter are soft-switching operation, high efficiency, reduced number of switches and low electromagnetic interference (EMI).

\subsubsection{Proposed Three-Phase AC-AC Converter}

The proposed direct three-phase ac-ac converter is shown in Fig. 5.1. In this figure, $C$ represents the primary compensation capacitor, $L$ is the primary self-inductance and $R_{e q}$ is the reflected resistance of the load at the secondary to the primary circuit. 


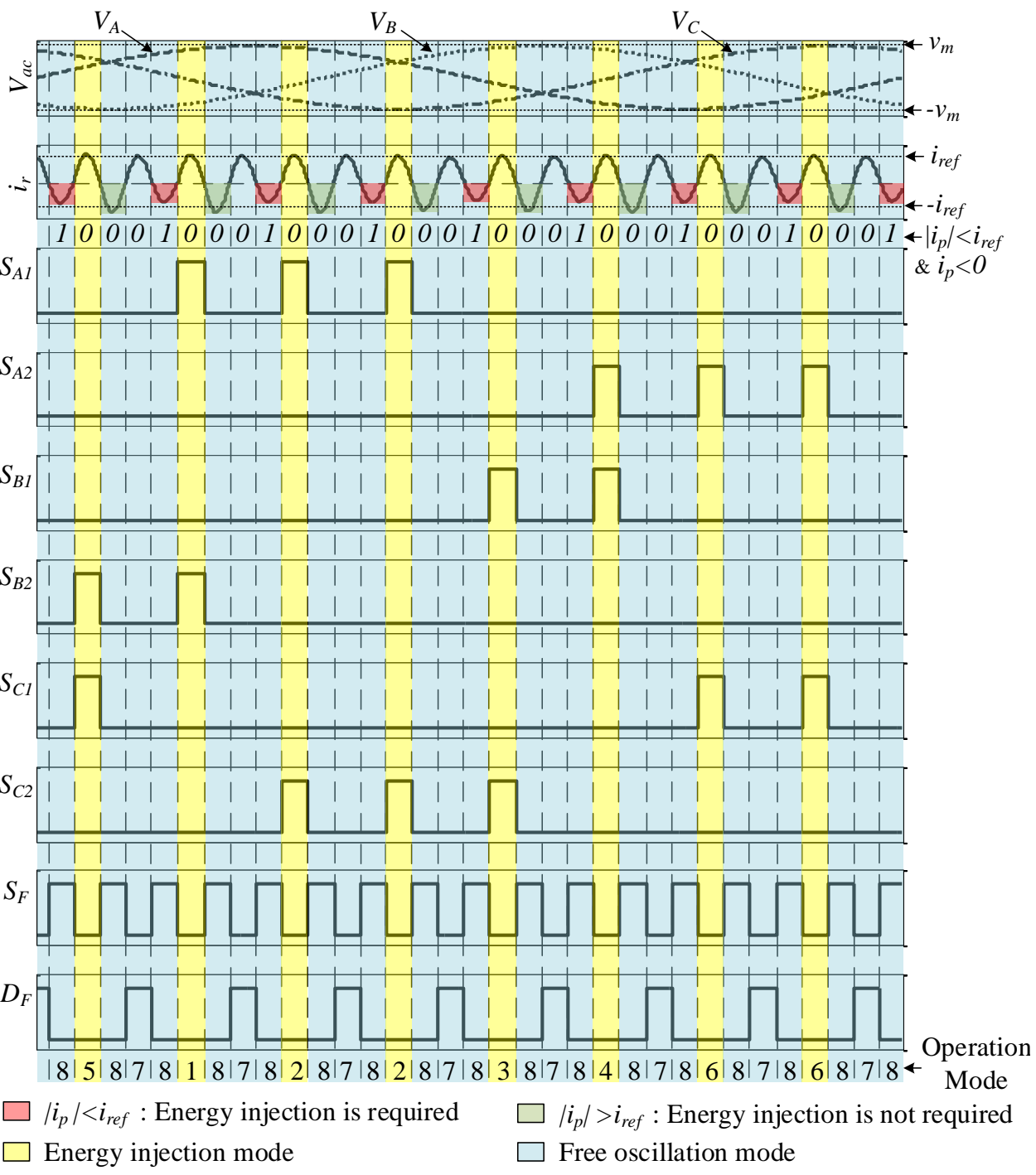

Figure 5.2: A conceptual plot of three-phase input voltages, resonant current and corresponding switching signals of the converter in different modes of operations.

The proposed converter consists of six reverse blocking switches and one regular switch (IGBT or MOSFET) which is in parallel with the resonant tank. A reverseblocking switch can be built using a series combination of an IGBT or a MOSFET with a diode. However, individual switches with reverse-blocking capability are now available in the market and have the advantage of total lower forward voltage and thereby, enable the converter to operate with higher efficiency. 
In [124], a control strategy for a single phase ac-ac converter based on energy injection and free oscillation of the resonant circuit is presented. This control method is further developed in this study and is applied for three different control modes, which are resonant current regulation control, power regulation control and resonant voltage regulation control. The control modes are all based on zero current switching (ZCS) operation. Since the proposed converter is based on the resonant current zero crossing points, the operating frequency of the converter is equal to the resonant current frequency (natural damped frequency, which is described in Section 5.1.2). Therefore, the operating frequency of the converter is determined by the circuit parameters. In a dynamic IPT system, the primary and secondary self-inductances are fixed by the track/coil parameters, such as size and number of turns in the coil. In practice, although the primary's position relative to secondary affects the mutual inductance, it has a very small effect on self-inductances, due to the inherently large air gaps required in electric vehicle (EV) dynamic charging systems, such variations are minimized. Therefore, self-inductances of the primary ( $L$ in Fig. 5.1 ) and secondary are constant. This ensures the performance of the converter in dynamic IPT systems.

The operation of the converter for each control mode can be described in eight operation modes, which are presented in Tables 5.1, 5.2 and 5.3. The operation modes 1 to 6 are energy injection modes in which energy is injected to the LC tank, and the operation modes 7 and 8 are free oscillation modes in which the LC tank continues its resonant oscillation. The transition of different modes of operation occurs at current zero-crossing points. Each mode starts at a resonant current zerocrossing, and continues for a half cycle until the next resonant current zero-crossing. This operation mode transition is determined based on the state of the circuit, and the reference values for the resonant current, voltage, and the output power. 


\subsubsection{Current Regulation Control Mode}

The resonant current regulation plays a key role in the power transfer performance of an IPT system. Since the resonant current amplitude is proportional to the amount of injected energy to the LC tank, the resonant current regulation control can be achieved by continuously changing the operation mode of the converter from energy injection modes (increasing the resonant current) to free oscillation modes (decreasing the resonant current), and vice versa. Using this strategy, the resonant current can be regulated around a user-defined reference current. This is carried out by comparing the peak output resonant current, $i_{p}$ to the reference current $\left(i_{\text {ref }}\right)$ at each current zero-crossing point. The $i_{p}$ is measured in each half cycle of the resonant current. If $i_{p}$ is negative and its absolute value is less than $i_{r e f}\left(i_{p}<0\right.$ and $\left.\left|i_{p}\right|<i_{r e f}\right)$, an energy injection to the LC tank is required for the next half cycle to increase the resonant current. According to Table 5.1, the converter should enter one of the energy injection modes 1 to 6 , depending on the three-phase input voltages. Moreover, if $i_{p}$ is positive or its absolute value is more than $i_{r e f}\left(i_{p}>0\right.$ or $\left.\left|i_{p}\right|>i_{\text {ref }}\right)$, the converter should enter one of the free oscillation modes 7 and 8 . A conceptual plot of three-phase input voltages, resonant current and corresponding switching signals of the converter in different modes of operations is presented in Fig. 5.2. Also, Fig. 5.3 demonstrates the resonant current path in the proposed converter in 8 modes of operation.

In each energy-injection mode, the LC tank terminals are switched between the most positive and the most negative input lines. According to Table 5.1, the switching is performed using six reverse-blocking switches, $S_{A 1}, S_{A 2}, S_{B 1}, S_{B 2}, S_{C 1}$ and $S_{C 2}$, which are used to switch the three-phase input lines to the output during modes 1 to 6 , based on the measured input voltages. It should be noted that the energy injection always occurs in positive half-cycles of the resonant current. In 
free-oscillation modes, the negative half-cycles of the resonant current are conducted through the parallel switch $S_{F}$ (mode 8) and the positive half-cycles of the resonant current are conducted through the intrinsic body diode $D_{F}$ (mode 7 ). It should be noted that negative half-cycles are always free-oscillation modes; therefore $S_{F}$ is switched at the rate of the resonance frequency. Since the resonant current becomes negative after any mode from 1 to 7 , mode 8 always occurs after any other mode of operation.

\subsubsection{Voltage Regulation Control Mode}

The voltage limit in the LC tank and particularly in the compensation capacitor is of great importance. This voltage limit is mostly limited to the insulation level of the primary coils/tracks and voltage rating of the compensation capacitor. The voltage regulation control can be achieved using an approach similar to the current regulation control mode. In Section 5.1.2, it is shown that the peak resonant voltage occurs in each resonant current zero-crossing. Therefore, the resonant voltage can be measured in each current zero-crossing and peak voltage detection is not required.

In voltage regulation control mode, if the peak resonant voltage is negative and its absolute value is lower than the reference voltage $\left(v_{p}<0\right.$ and $\left.\left|v_{p}\right|<v_{r e f}\right)$, then according to Table 5.2, the circuit will enter one of the energy injection modes 1 to 6 , depending on the three-phase input voltages. Therefore, energy will be injected to the LC tank for a half cycle to increase the resonant voltage, and the LC tank terminals are switched between the most positive and the most negative input lines. The switching is performed using six switches, $S_{A 1}, S_{A 2}, S_{B 1}, S_{B 2}, S_{C 1}$ and $S_{C 2}$, which are used to switch the three-phase input lines to the output during modes 1 to 6 , according to Table 5.2 and based on the measured input voltages. Mode 7 occurs when the peak voltage is negative and its absolute value is higher than the 

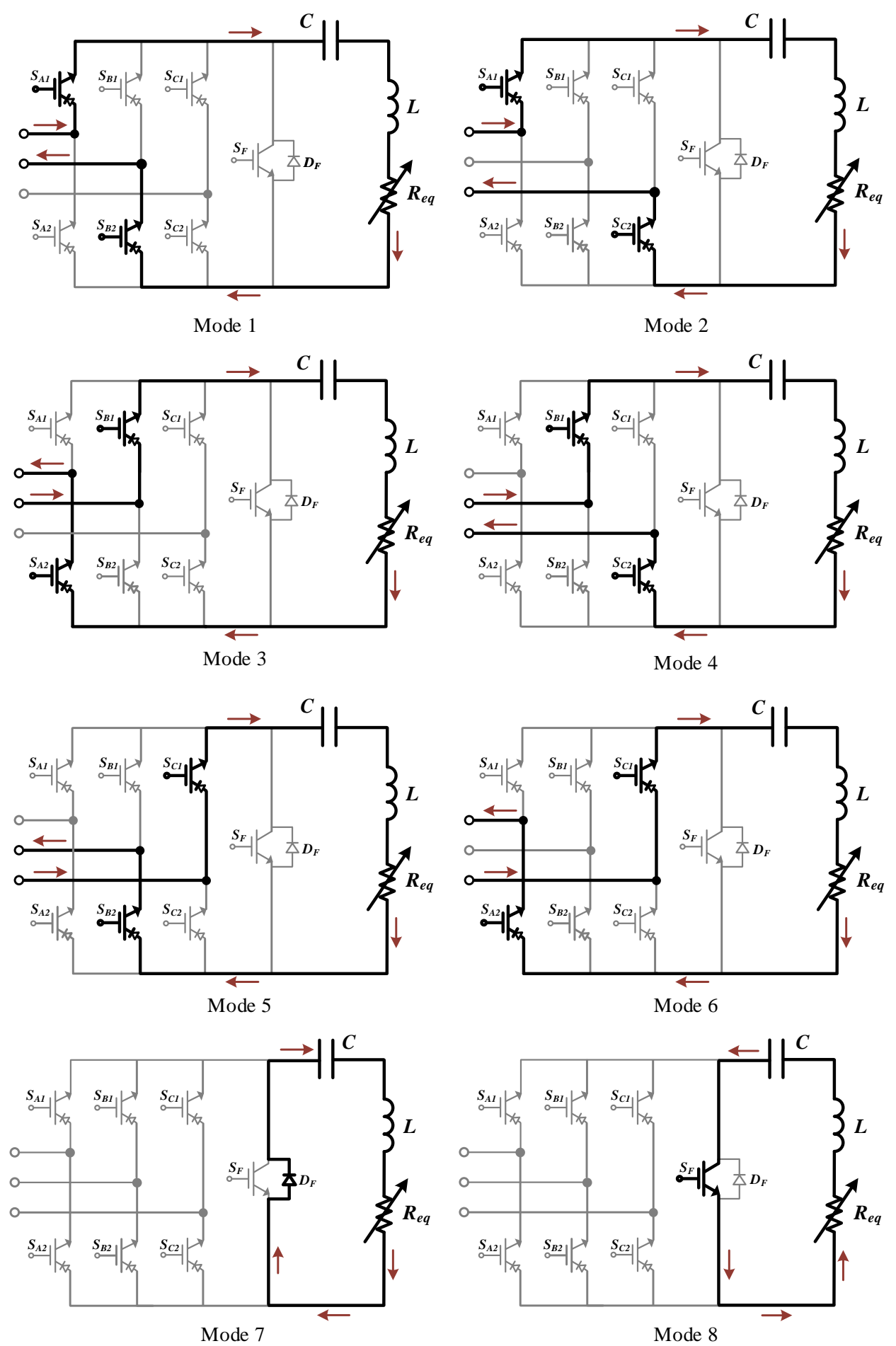

Figure 5.3: The current path in the proposed converter in eight modes of operation. 
Table 5.1: Switching States in different Modes of Operation in Current Regulation Control Mode.

\begin{tabular}{llll}
\hline Mode & $\begin{array}{l}\text { Resonant Cur- } \\
\text { rent }\end{array}$ & $\begin{array}{l}\text { Input } \\
\text { ages }\end{array}$ & $\begin{array}{l}\text { Conducting } \\
\text { Switches }\end{array}$ \\
\hline 1 & $i_{p}<0,\left|i_{p}\right|<i_{\text {ref }}$ & $V_{b}<V_{c}<V_{a}$ & $S_{A 1}, S_{B 2}$ \\
2 & $i_{p}<0,\left|i_{p}\right|<i_{\text {ref }}$ & $V_{c}<V_{b}<V_{a}$ & $S_{A 1}, S_{C 2}$ \\
3 & $i_{p}<0,\left|i_{p}\right|<i_{\text {ref }}$ & $V_{a}<V_{c}<V_{b}$ & $S_{B 1}, S_{A 2}$ \\
4 & $i_{p}<0,\left|i_{p}\right|<i_{\text {ref }}$ & $V_{c}<V_{a}<V_{b}$ & $S_{B 1}, S_{C 2}$ \\
5 & $i_{p}<0,\left|i_{p}\right|<i_{\text {ref }}$ & $V_{b}<V_{a}<V_{c}$ & $S_{C 1}, S_{B 2}$ \\
6 & $i_{p}<0,\left|i_{p}\right|<i_{\text {ref }}$ & $V_{a}<V_{b}<V_{c}$ & $S_{C 1}, S_{A 2}$ \\
7 & $i_{p}<0,\left|i_{p}\right|>i_{r e f}$ & - & $D_{F}$ \\
8 & $i_{p}>0$ & - & $S_{F}$ \\
\hline
\end{tabular}

Table 5.2: Switching States in different Modes of Operation in Voltage Regulation Control Mode.

\begin{tabular}{llll}
\hline Mode & $\begin{array}{l}\text { Resonant Voltage } \\
\text { \& Current }\end{array}$ & $\begin{array}{l}\text { Input } \\
\text { ages }\end{array}$ & $\begin{array}{l}\text { Conducting } \\
\text { Switches }\end{array}$ \\
\hline 1 & $v_{p}<0,\left|v_{p}\right|<v_{r e f}$ & $V_{b}<V_{c}<V_{a}$ & $S_{A 1}, S_{B 2}$ \\
2 & $v_{p}<0,\left|v_{p}\right|<v_{r e f}$ & $V_{c}<V_{b}<V_{a}$ & $S_{A 1}, S_{C 2}$ \\
3 & $v_{p}<0,\left|v_{p}\right|<v_{r e f}$ & $V_{a}<V_{c}<V_{b}$ & $S_{B 1}, S_{A 2}$ \\
4 & $v_{p}<0,\left|v_{p}\right|<v_{r e f}$ & $V_{c}<V_{a}<V_{b}$ & $S_{B 1}, S_{C 2}$ \\
5 & $v_{p}<0,\left|v_{p}\right|<v_{r e f}$ & $V_{b}<V_{a}<V_{c}$ & $S_{C 1}, S_{B 2}$ \\
6 & $v_{p}<0,\left|v_{p}\right|<v_{r e f}$ & $V_{a}<V_{b}<V_{c}$ & $S_{C 1}, S_{A 2}$ \\
7 & $v_{p}<0,\left|v_{p}\right|>v_{r e f}$ & - & $D_{F}$ \\
8 & $i_{p}>0$ & - & $S_{F}$ \\
\hline
\end{tabular}

reference voltage $\left(v_{p}<0\right.$ and $\left.\left|v_{p}\right|>v_{\text {ref }}\right)$ and therefore energy injection to LC tank should be avoided for a half cycle to decrease the resonant voltage. In this mode, the LC tank enters a free oscillation state and the resonant current is positive, which is conducted through the intrinsic body diode $\left(D_{F}\right)$ of the parallel switch $\left(S_{F}\right)$ as shown in Fig. 5.3 for mode 7 . In mode 8, the resonant current is negative and the switch $S_{F}$ is on. Since the resonant current becomes negative after any mode between 1 to 7 , mode 8 always occurs after any other mode of operation.

\subsubsection{Power Regulation Control Mode}

In dynamic IPT systems due to inherent variations in the load power transfer control is important. The power input regulation control can be achieved using an approach similar to current regulation control method. The peak current $\left(i_{p}\right)$ and the input 
Table 5.3: Switching States in different Modes of Operation in Power Regulation Control Mode.

\begin{tabular}{llll}
\hline Mode & $\begin{array}{l}\text { Output Power \& } \\
\text { Resonant Current }\end{array}$ & $\begin{array}{l}\text { Input } \\
\text { ages }\end{array}$ & $\begin{array}{l}\text { Volt- } \\
\text { Switches }\end{array}$ \\
\hline 1 & $P_{\text {out }}<P_{\text {ref }}, i_{p}<0$ & $V_{b}<V_{c}<V_{a}$ & $S_{A 1}, S_{B 2}$ \\
2 & $P_{\text {out }}<P_{\text {ref }}, i_{p}<0$ & $V_{c}<V_{b}<V_{a}$ & $S_{A 1}, S_{C 2}$ \\
3 & $P_{\text {out }}<P_{\text {ref }}, i_{p}<0$ & $V_{a}<V_{c}<V_{b}$ & $S_{B 1}, S_{A 2}$ \\
4 & $P_{\text {out }}<P_{\text {ref }}, i_{p}<0$ & $V_{c}<V_{a}<V_{b}$ & $S_{B 1}, S_{C 2}$ \\
5 & $P_{\text {out }}<P_{\text {ref }}, i_{p}<0$ & $V_{b}<V_{a}<V_{c}$ & $S_{C 1}, S_{B 2}$ \\
6 & $P_{\text {out }}<P_{\text {ref }}, i_{p}<0$ & $V_{a}<V_{b}<V_{c}$ & $S_{C 1}, S_{A 2}$ \\
7 & $P_{\text {out }}>P_{\text {ref }}, i_{p}<0$ & - & $D_{F}$ \\
8 & $i_{p}>0$ & - & $S_{F}$ \\
\hline
\end{tabular}

voltage $\left(V_{i n}\right)$ are measured. Considering the fact that all negative half-cycles are free oscillation modes, and in free oscillation modes the input voltage is zero $\left(V_{\text {in }}=0\right)$, the average output power $\left(P_{i n}\right)$ for a full-cycle $(T)$ can be calculated as below:

$$
P_{\text {out }}=\int_{T} i_{p} V_{\text {in }}=\frac{1}{\pi} i_{p} V_{\text {in }}
$$

In this control mode, in each current zero crossing $P_{\text {out }}$ is compared to a reference power $\left(P_{\text {ref }}\right)$ and if the average output power $\left(P_{\text {out }}\right)$ in one half cycle is lower than the reference power $\left(P_{r e f}\right)$, the circuit will enter one of the energy injection modes 1 to 6 , depending on the three-phase input voltages based on Table 5.3. Therefore, energy will be injected to the LC tank in the next half cycle to increase the resonant current, and the LC tank terminals are switched between the most positive and the most negative input lines. According to Table 5.3., the switching is performed using six switches, $S_{A 1}, S_{A 2}, S_{B 1}, S_{B 2}, S_{C 1}$ and $S_{C 2}$, which are used to switch the threephase input lines to the output during modes 1 to 6 , based on the measured input voltages. Mode 7 occurs when the average output power $\left(P_{\text {out }}\right)$ is higher than the reference power $\left(P_{r e f}\right)$; therefore, energy injection to LC tank should be avoided for a half cycle to decrease the resonant current. In this mode, the LC tank enters a free oscillation state and the resonant current is positive, which is conducted through the intrinsic body diode $\left(D_{F}\right)$ of the parallel switch $\left(S_{F}\right)$ as shown in Fig. 5.3 for mode 


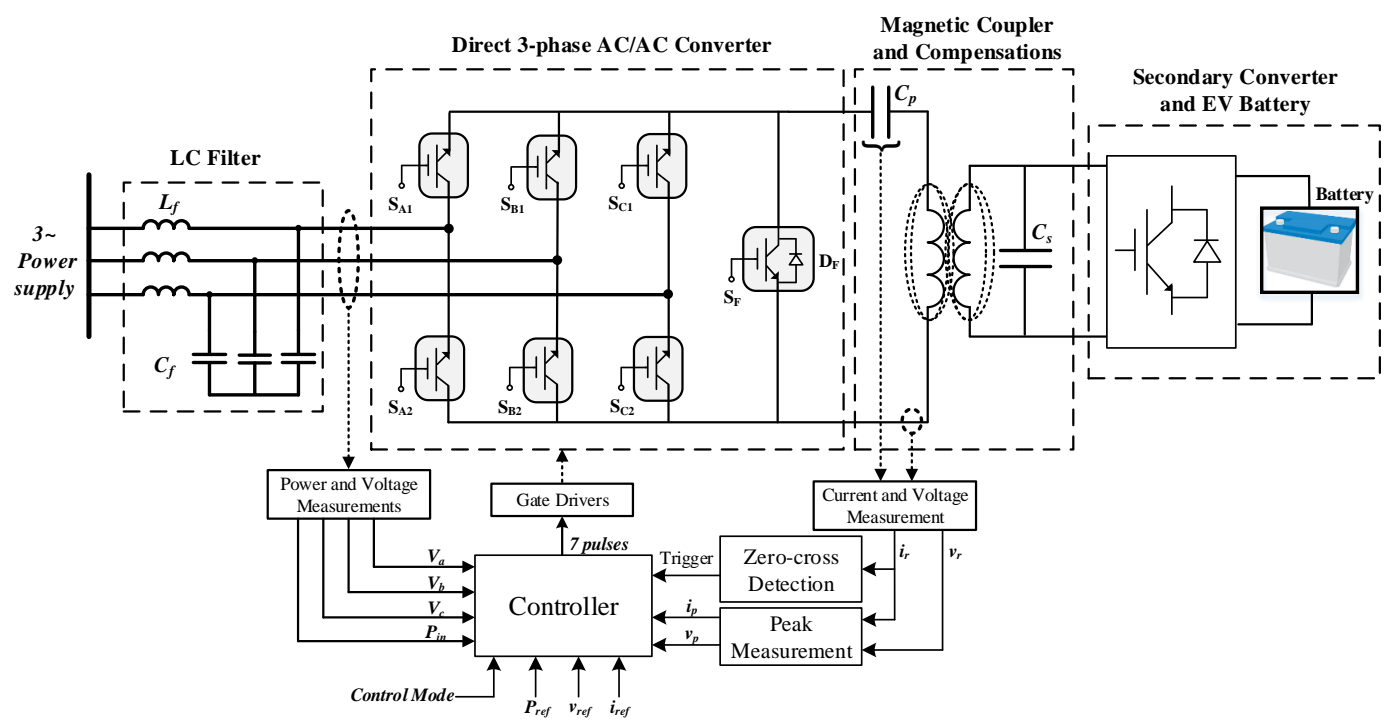

Figure 5.4: The IPT model and its components simulated in MATLAB/Simulink.

7. In mode 8 , the resonant current is negative and the switch $S_{F}$ is on. Since the resonant current becomes negative after any mode between 1 to 7 , mode 8 always occurs after any other mode of operation.

\subsubsection{Theoretical Analysis}

The differential equation of a LC tank with primary self-inductance of $L$, and compensation capacitor $C$ with an equivalent resistance of $R$ can be expressed as,

$$
L \frac{d i}{d t}+R_{e q} i+\frac{1}{C} \int_{0}^{t} i d t+v_{c}(0)=V_{t}
$$

where $i$ is the resonant current, $v_{c}$ is the voltage of the compensation capacitor and $V_{t}$ is the input voltage. Equation (5.2) can be rewritten as the following second order differential equation:

$$
\frac{d^{2} i}{d t^{2}}+\frac{R_{e q}}{L} \frac{d i}{d t}+\frac{1}{L C} i=0
$$


where the initial conditions of the circuit are:

$$
\begin{aligned}
i(0) & =0 \\
L \frac{d i}{d t}(0) & =V_{t}-v_{c}(0)
\end{aligned}
$$

The solution of (5.3) based on initial conditions in (5.4) is derived as:

$$
i=K e^{-t / \tau} \sin (\omega t)
$$

where the natural damped frequency $\omega=\sqrt{\omega_{0}^{2}-\alpha^{2}}$, resonant frequency $\omega_{0}=$ $1 / \sqrt{L C}$, damping coefficient $\alpha=R_{e q} / 2 L$, damping time constant $\tau=2 L / R$ and the coefficient $K$ is expressed as:

$$
K=\frac{V_{t}-v_{c}(0)}{\omega L}
$$

Equation (5.5) shows that the peak current decreases exponentially with a time constant of $\tau$ and (5.6) shows that the value of $K$ changes in each half cycle depending on the input voltage and initial voltage of the compensation capacitor. It should be noted that in the free oscillation modes the input voltage is zero $\left(V_{t}=0\right)$. Also the compensation capacitor voltage can be expressed as:

$$
v_{c}(t)=v_{c}(0)+\frac{K \tau}{C\left(1+\tau^{2} \omega^{2}\right)}\left(\tau \omega-e^{-t / \tau}[\sin (\omega t)+\tau \omega \cos (\omega t)]\right)
$$

The resonant current and voltage equations (5.5) and (5.7) can be used for finding the peak values of current and voltage in each half cycle. In order to find the peak value of the resonant current $i_{n}$, which occurs at the time $t_{n}$ corresponding to the $n$th current peak, the following equation can be solved to find the extremum points of the resonant current:

$$
\frac{d i}{d t}=K e^{-t / \tau}\left[\omega \cos (\omega t)-\frac{1}{\tau} \sin (\omega t)\right]=0
$$

By simplifying (5.8) the following equations are derived:

$$
\tan \left(\omega t_{n}\right)=\tau \omega
$$




$$
t_{n}=\frac{\operatorname{atan}(\tau \omega)+n \pi}{\omega}
$$

therefore the $n$th peak value of the resonant current can be calculated using (5.5) and (5.10) as:

$$
i_{n}=K e^{-\frac{\operatorname{atan}(\tau \omega)+n \pi}{\tau \omega}}(-1)^{n} \frac{\tau \omega}{\sqrt{1+(\tau \omega)^{2}}}
$$

Similarly the peak values of the resonant voltage can be found using (5.7) a,

$$
\begin{aligned}
\frac{d v_{c}}{d t}= & -\frac{K \tau e^{-t / \tau}}{C\left(1+\tau^{2} \omega^{2}\right)}\left(\left[\omega \cos (\omega t)-\tau \omega^{2} \sin (\omega t)\right]\right. \\
& \left.-\frac{1}{\tau}[\sin (\omega t)+\tau \omega \cos (\omega t)]\right)=0
\end{aligned}
$$

Equation (5.12) can be simplified as following set of equations:

$$
\begin{gathered}
\frac{d v_{c}}{d t}=\frac{K}{C} e^{-t / \tau} \sin (\omega t)=0 \\
\sin \left(\omega t_{n}\right)=0 \\
t_{n}=\frac{n \pi}{\omega}
\end{gathered}
$$

Based on (5.5), (5.13), (5.14) and (5.15) it can be seen that in each resonant current zero-crossing, resonant voltage is exactly in its peak. Since control modes, which are presented in Section 5.1.1 are all based on resonance current zero-crossing points, the voltage regulation control mode can be established on peak values of resonant voltage in each current zero-crossing.

Using (5.5), the resonant current in a time period composed of both energy injection and free oscillation modes can be expressed as follows:

$$
i(t)= \begin{cases}K_{i} e^{-t / \tau} \sin (\omega t) & 0<t<\frac{\pi}{\omega} \\ K_{f} e^{-t / \tau} \sin (\omega t) & \frac{\pi}{\omega}<t<\frac{2 m \pi}{\omega}\end{cases}
$$

where $m$ denotes the number of cycles, which is composed of one energy injection half cycle and $2 m-1$ free oscillation half cycles, $K_{i}$ and $K_{f}$ are coefficients of 
(5.5) in the first energy injection and free oscillation half cycles respectively, can be calculated using (5.6) and (5.7) as follows:

$$
\begin{gathered}
K_{i}=\frac{1}{\omega L}\left[V_{t}-v_{c}(0)\right] \\
K_{f}=\frac{1}{\omega L}\left[v_{c}\left(\frac{\pi}{\omega}\right)\right]=\frac{1}{\omega L}\left[v_{c}(0)+\frac{K_{i} \tau^{2} \omega}{C\left(1+\tau^{2} \omega^{2}\right)}\left(1+e^{-\pi / \tau \omega}\right)\right]
\end{gathered}
$$

By assuming $i_{\text {ref }}$ as the reference current, using (5.11) and (5.12) the number of cycles that the next energy injection should occur $(m)$ can be calculated as follows:

$$
m=\frac{1}{\pi}\left\lfloor\tau \omega \ln \left(\frac{K_{f} \tau \omega}{i_{\text {ref }} \sqrt{1+(\tau \omega)^{2}}}\right)+\arctan (\tau \omega)\right\rfloor
$$

Equation (5.19) predicts the number of cycles that the LC tank will continue its free oscillation mode, after an energy-injection mode, as a function of initial condition $\left(K_{f}\right)$, circuit parameters $(\tau \omega)$ and the reference current $i_{r e f}$. A duty-cycle can be defined as the ratio of the number of energy injection modes to the number of free-oscillation modes in the time interval between two successive energy injection modes, and can be written as,

$$
D_{i}=\frac{1}{m+1}=\frac{f_{i n j}}{f_{r}}
$$

where $f_{i n j}$ is the switching frequency of the energy-injection modes and $f_{r}$ is the resonance frequency of the LC tank. The duty-cycle $D_{i}$, is a measure that represents the energy demand for the LC tank. For example in Fig. 5.2, this measure is $D_{i}=0.5$, which shows that only half of the cycles energy needs to be injected to the LC tank.

\subsubsection{Converter Loss Analysis}

The power loss of the proposed converter can be calculated by evaluating the conduction and switching losses of the power switches in different modes of operation. 
Table 5.4: Typical values for parameters used for theoretical converter loss calculation.

\begin{tabular}{lll}
\hline Parameter & Description & $\begin{array}{l}\text { Typical } \\
\text { Value }\end{array}$ \\
\hline$V_{F}$ & Switch forward voltage & $1.5 \mathrm{~V}$ \\
$R_{F}$ & Equivalent ON-state resistance & $0.08 \Omega$ \\
$C_{o s s}$ & Output capacitance & $250 \mathrm{pF}$ \\
$E_{\text {on }}+E_{\text {off }}$ & Switching volt-ampere crossover energy losses & $2 \mathrm{~mJ}$ \\
$V_{F D}$ & Diode forward voltage & $1.8 \mathrm{~V}$ \\
$E_{r r}$ & Reverse recovery energy loss & $200 \mu \mathrm{J}$ \\
\hline
\end{tabular}

The loss of each power switch is composed of switching and conduction losses and can be written as,

$$
\begin{aligned}
P_{S x}=P_{S c o n}+P_{S s w}=\left[V_{F} I_{a v g}+R_{F} I_{r m s}^{2}\right] & T_{\text {con }} f_{s w} \\
& +\left[\left(E_{o n}+E_{o f f}\right)+\frac{1}{2} C_{o s s} V_{i n}^{2}\right] f_{s w}
\end{aligned}
$$

where $P_{S c o n d}$ and $P_{S s w}$ are the conduction and switching losses of the switch $S_{x}$ respectively, $V_{F}$ is the forward voltage of the power switch (in power MOSFETs, $\left.V_{F}=0\right), R_{F}$ is the equivalent resistance of the switch during on state, $I_{\text {avg }}$ and $I_{r m s}$ are the mean and RMS values of the conducted current respectively, $T_{\text {con }}$ is the conduction time of the switch, $f_{s w}$ is the switching frequency, $E_{\text {on }}$ and $E_{\text {off }}$ are volt-ampere crossover energy losses during the switch turn-on and turn-off transitions respectively, $C_{\text {oss }}$ is the output capacitance of the switch, and $V_{\text {in }}$ is the input voltage. Since in the converter benefits from ZCS switching is always performed, the switching losses from volt-ampere crossover are minimized and thus, are relatively low. Therefore, the conduction losses dominate, followed by the losses due to $C_{o s s}$ (output losses). The losses associated with any diode in the converter are composed of conduction and reverse recovery losses and can be calculated as below:

$$
P_{D x}=P_{D c o n d}+P_{D r r}=V_{F D} I_{a v g} T_{c o n} f_{s w}+E_{r r} f_{s w}
$$

where $P_{D \text { cond }}$ and $P_{D r r}$ are the conduction and reverse recovery losses of the diode $D_{x}$ respectively, $V_{F D}$ is the forward voltage of the diode and $E_{r r}$ is the reverse recovery 
energy of the diode. The losses of the converter can be calculated by calculation of the losses associated with energy-injection and free-oscillation modes separately, considering the fact that the switching frequency of all the switches are $f_{i n j}=D_{i} f_{r}$, except $S_{F}$ which its switching frequency is $f_{r}$. In each energy injection mode (modes 1 to 6) two reverse blocking switches are involved; therefore, the losses associated with energy injection modes $\left(P_{i n j}\right)$ can be described as,

$$
P_{i n j}=2 D_{i}\left(P_{S x}+P_{D x}\right)
$$

It should be noted that if the switches $S_{A 1}, S_{A 2}, S_{B 1}, S_{B 2}, S_{C 1}$ and $S_{C 2}$ are switches with built-in reverse blocking capability, $P_{D x}$ loss is eliminated in (5.23), and as a result the efficiency of the converter will be increased. Since $S_{F}$ and its body diode $D_{F}$ are the only switches, involved in free oscillation modes, the losses associated with free oscillation modes $\left(P_{\text {osc }}\right)$ can be calculated as follows:

$$
P_{o s c}=P_{S x}+D_{i} P_{D x}
$$

Finally the total dissipated power can be described as follows:

$$
P_{l o s s}=P_{i n j}+P_{o s c}
$$

Typical values for high power switches and diodes for 50 A output current are presented in Table 5.4. However, for different values of current, these typical values should be modified accordingly. Fig. 5.5 presents the efficiency of the converter versus resonant current and switching frequency, which is calculated based on Table 5.4 and equations (5.21)-(5.25). This figure shows that the efficiency of the converter increases as the resonant current increases, and any change in the switching frequency, does not affect the efficiency significantly in high resonant currents. Also, the maximum efficiency of the converter is $96.6 \%$. 


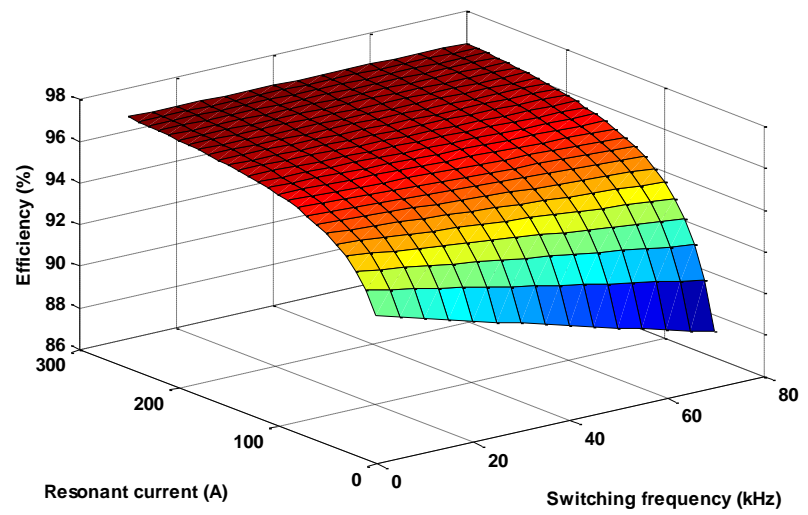

Figure 5.5: Efficiency of the converter vs. switching frequency $\left(f_{s w}\right)$ and resonant current $\left(i_{r}\right)$, calculated analyticaly.
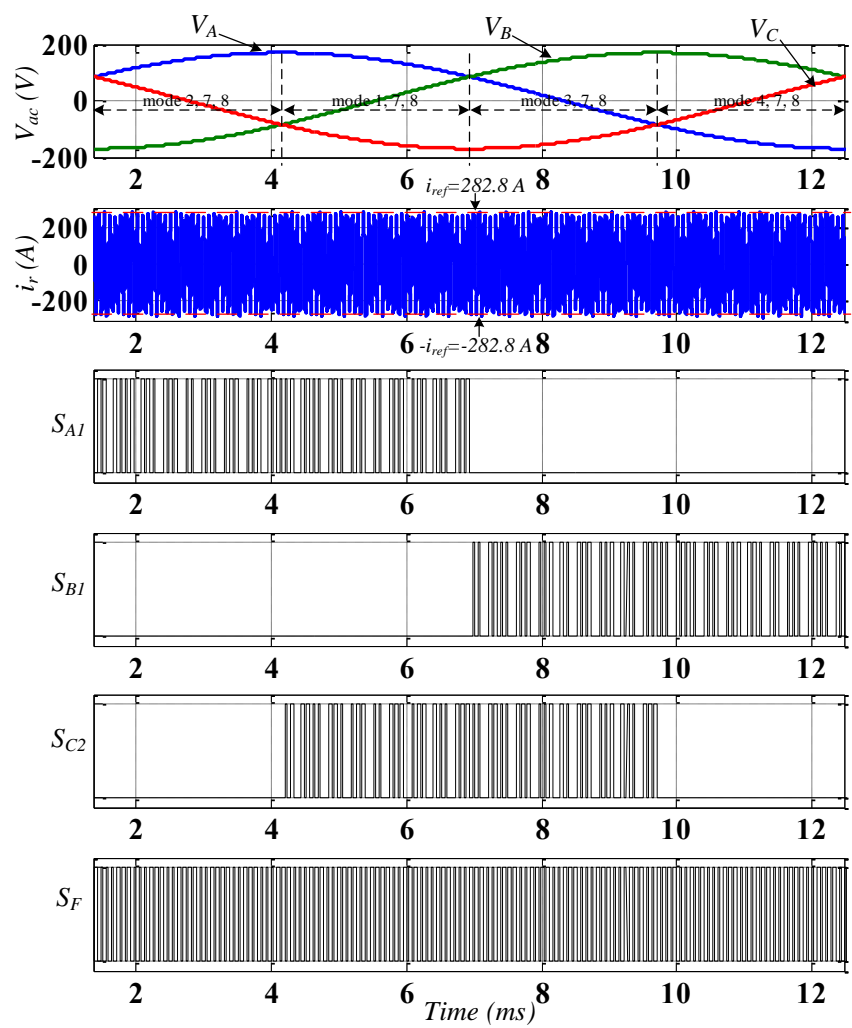

Figure 5.6: Simulation results of the IPT system with $i_{r e f}=282.8 \mathrm{~A}$.

\subsubsection{Simulation Results}

The proposed three-phase converter, which is presented in Fig. 5.1, is simulated using MATLAB/Simulink. The IPT model is shown in Fig. 5.4. This model is 


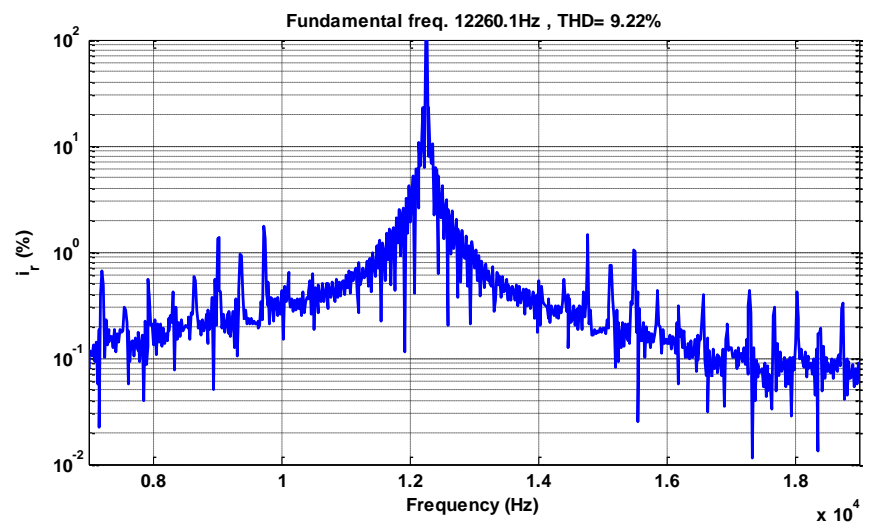

Figure 5.7: Frequency spectrum of the resonant current shown in Fig. 5.6.

composed of a three-phase power supply with an output LC filter, the primary three-phase ac-ac converter, the primary and secondary magnetic structures with their corresponding compensation capacitors and the secondary load, which is a battery charger of an electric vehicle. The controller of the primary converter and its components are also shown in Fig. 5.4. The measurements include the threephase input voltage and the output resonant current of the LC tank. The controller is triggered in each resonant current zero-crossing, and based on the voltage and current measurements the switching state of the of the converter is determined. The switching signals of the converter does not change until the next current zerocrossing.

The self-inductances of the primary and secondary are each $168 \mu H$, where each have a $1 \mu F$ compensation capacitor and thereby, the operating frequency of the converter, which is equal to the resonance frequency of the LC tank would be $12.28 \mathrm{kHz}$. The line-to-line voltage of the three-phase supply is $208 \mathrm{~V}$. The current regulation control mode is enabled with 282.8 A (200 Arms) reference current. The simulation results including the three-phase input voltages and their corresponding modes of operation, the output resonant current and corresponding switching signals of $S_{A 1}, S_{B 1}, S_{C 2}, S_{F}$, are shown in Fig. 5.6. Also, the frequency spectrum of the 
output resonant current is shown in Fig. 5.7. As it can be seen in Figs. 5.6 and 5.7, the current is fully regulated around the reference current and its THD is $9.22 \%$. The active and reactive power calculations show that the total output power is $18.4 \mathrm{~kW}$ and the fundamental reactive power is zero $\left(Q_{1}=0\right)$ and therefore, the displacement power factor is unity $(D P F=1)$. However, due to higher order harmonics in the three-phase input voltages and currents, the true power factor is 0.76 . Using the specifications given in Table 5.4 for the switches of the converter, the efficiency of the converter is calculated based on (5.21)-(5.25) through the simulation, which is $96.2 \%$.

\subsubsection{Experimental Results}

The experimental study is performed on a case study IPT system, which is shown in Fig. 5.8. This system is composed of two circular magnetic structures with 700 mm diameter and $200 \mathrm{~mm}$ air gap. The circular power pads are composed of coils, ferrite bars, and aluminum shields. The proposed three-phase matrix converter is used as the primary converter with a series-parallel topology, which is shown in Fig. 5.4 is implemented where, the self-inductance of each circular pad is $168 \mu H$, and 1 $\mu F$ compensation capacitors are used in both primary and secondary circuits. The

line voltage of the three-phase power supply is $40 \mathrm{~V}$. In this experimental study, series combination of a MOSFET (IRF3205) with a diode (DSEP 30-12A) is used to make the reverse the blocking switches. However, switches with built-in reverse blocking capability are available in the market (e.g. IXRH-40N120). The switch $S_{F}$ is also an IRF3205 MOSFET, and its body diode is used as the diode $D_{F}$. A STM32F4-discovery board with an ARM Cortex-M4 $168 \mathrm{MHz}$ DSP is used as the main controller. The resonant current regulation and output power regulation 


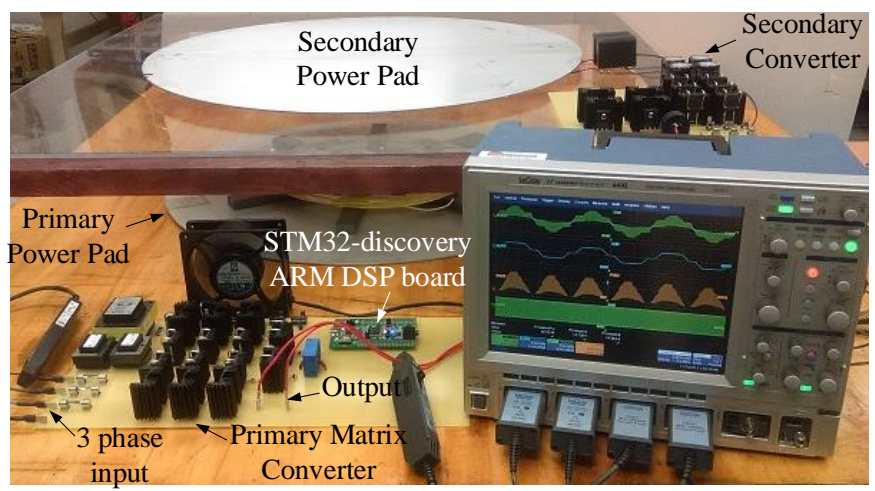

Figure 5.8: The case study IPT system with circular power pads.

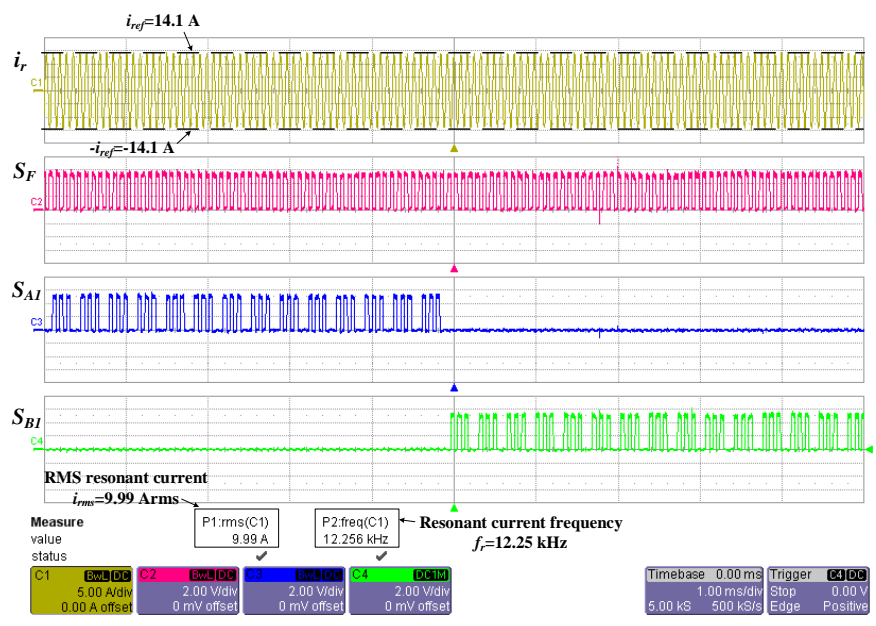

Figure 5.9: Experimental results on the case study IPT system: Output resonant current and corresponding switching signals.

control strategies are studied on the case study IPT system, which are described in the following sections:

\subsubsection{Current Regulation Control}

The resonant current regulation control with 14.1 A (10 Arms) reference current is used to regulate the resonance current. Figure 10 shows the output resonant current and corresponding switching signals of $S_{A 1}, S_{B 1}$ and $S_{F}$ switches during the transition of the most positive phase in voltage from phase A to phase B. The operating 


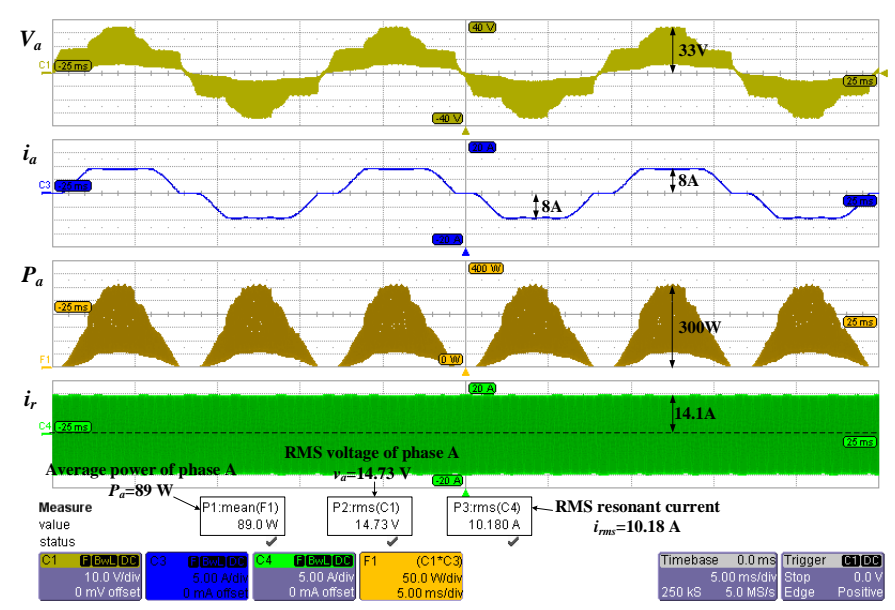

Figure 5.10: Experimental results on the case study IPT system: input voltage $\left(V_{a}\right)$, input current $\left(i_{a}\right)$, and power $\left(P_{a}\right)$ for phase $\mathrm{A}$ and output resonant current $\left(i_{r}\right)$.

frequency of the converter is $12.25 \mathrm{kHz}$, which has a small discrepancy compared to the theoretical resonance frequency $(12.28 \mathrm{kHz})$. The waveforms of the input voltage $\left(V_{a}\right)$, input current $\left(i_{a}\right)$, input power $\left(P_{\text {out }}\right)$ for phase A and output resonant current $\left(i_{r}\right)$ are shown in Fig. 5.10. As it can be seen, the resonant current is fully regulated around the reference current. However, the input voltage has high frequency harmonics which will reduce the true power factor of the converter as a result it also will reduce the power transfer efficiency of the converter. The frequency spectrum of the output resonant current is measured experimentally, which is shown in Fig. 5.11. Calculations show that the THD of the output resonant current is $14.3 \%$. The total output power is $267 \mathrm{~W}$ ( $89 \mathrm{~W}$ from each phase). The input power factor measurements show that the true power factor is 0.67 . However, the fundamental reactive power is zero and therefore, the displacement power factor is unity. Also, the measured loss of the converter is $31.5 \mathrm{~W}$ and consequently, the efficiency of the converter is $89.4 \%$ compared to $92.7 \%$ theoretical efficiency using (5.21)-(5.25). 


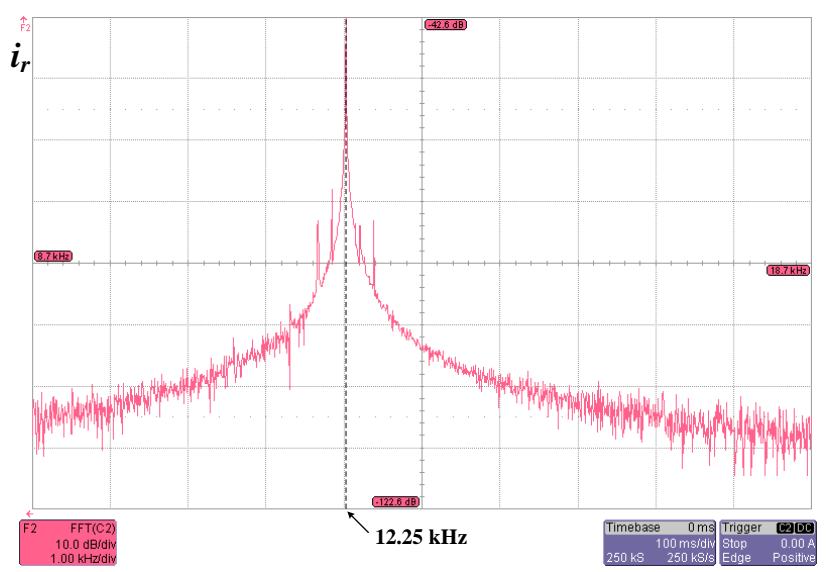

Figure 5.11: Frequency spectrum of the resonant current shown in Fig. 5.10.

The main limiting factors in the proposed converter are the speed of the controller (DSP board) and the response delay time of the resonant current measurement. In IPT applications high-frequency operation of the converter $(10-85 \mathrm{kHz})$ is essential to maximize the power transfer efficiency. On the other hand, in the proposed converter the current and voltage measurements using analog to digital conversion (ADC), with high sampling rate are required. Also, the implemented control strategy on the DSP, which consist of floating-point operations and comparisons, etc, along with ADC conversions, will take tens of clock cycles of the DSP to execute. As a result, a DSP with a high clock speed is essential. The maximum frequency that can be practically achieved using the DSP board (STM32F4-discovery ARM Cortex-M4 $168 \mathrm{MHz} \mathrm{DSP}$ ), is about $40 \mathrm{kHz}$. However, the proposed controller of the converter has the potential to be implemented based on analog circuit, which can significantly enhance the controller speed and resolve the need for a DSP.

Also, a Hall-effect current transducer "LA 55-P" is used for the resonant current measurement, which has a response delay less than $1 \mu \mathrm{s}$. Considering the fact that at least 20 samples in a full-cycle of the resonant current are required for a proper performance of the converter (without losing the zero-crossing points), the maximum 
frequency that practically can be achieved is about $50 \mathrm{kHz}$ based on the response time delay of the current measurement.

\subsection{Three-Phase AC-AC Matrix Converter with Simplified Bidirectional Power Control for Inductive Power Trans- fer Systems}

The use of direct three-phase ac-ac matrix converter with bidirectional power control for inductive power transfer (IPT) systems is proposed. The converter enables direct power conversion between low-frequency three-phase AC mains and high-frequency IPT systems. A digital power controller is designed and developed to regulate the power transfer rate at the desired level in both directions. A simplified circuit for the bidirectional power controller is presented which can be implemented with a few number of logic components or using a Field Programmable Gate Array (FPGA). The controller enables soft-switching operations and benefits from the resonance frequency tracking capability to maintain the high-efficiency power transmission at any operating conditions. The converter achieves bidirectional power transfer which is specifically useful for establishing grid-to-vehicle (G2V) and vehicle-to-grid (V2G) connections through inductive electric vehicle (EV) charging/discharging systems. Also, the proposed converter can be employed in dynamic IPT systems as it can cope with variations of the system. The controller design methodology, simulation analysis, and the preliminary experimental results of the proposed matrix converter on a case study inductive battery charging system are presented in detail.

In this section, the use of direct three-phase AC-AC matrix converter with a simplified power transfer control for IPT applications is proposed. Matrix converters 
are bidirectional which makes it suitable for IPT based grid-to-vehicle (G2V) and vehicle-to-grid (V2G) connections. A power controller is specifically designed for the converter to enable the bidirectional power transfer regulation at a desired level. The controller has the resonance frequency tracking capability to ensure maximum power transfer efficiency of in the system. Therefore, the proposed converter can be employed in dynamic IPT systems as it can cope into variations of the system. The converter benefits from soft-switching operations which significantly enhances the performance of the converter and reduces the complexity of the commutation between bidirectional switches. A simplified circuit for the power controller is presented which can be implemented with a few number of logic components or using a Field Programmable Gate Array (FPGA). The controller enables soft-switching operations and benefits from the resonance frequency tracking capability to maintain the high-efficiency power transmission at any operating conditions. The controller design methodology, simulation analysis, and the preliminary experimental results of the proposed matrix converter on a case study inductive battery charging system are presented in detail. The bidirectional power controller prototype is developed on an FPGA.

\subsubsection{Bidirectional Power Transfer Control for Three-Phase AC-AC Converter}

The AC-AC matrix converter which is comprised of six bidirectional switches is presented in Fig. 5.12. The IPT system is modeled as a series RLC circuit where $L$ is the transmitter inductance, $C$ is the series connected compensation capacitor, and $R_{e q}$ is the equivalent load resistance reflected to the primary. The RLC equivalent circuit model is derived by reflecting the secondary load to the primary side. In 


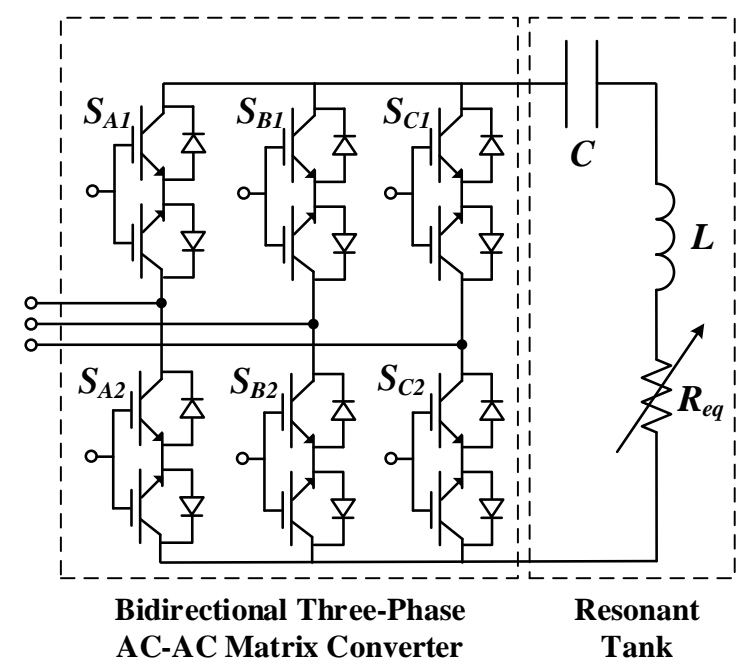

Figure 5.12: A direct three-phase to single-phase AC-AC matrix converter connected to an equivalent RLC circuit representing an IPT system.

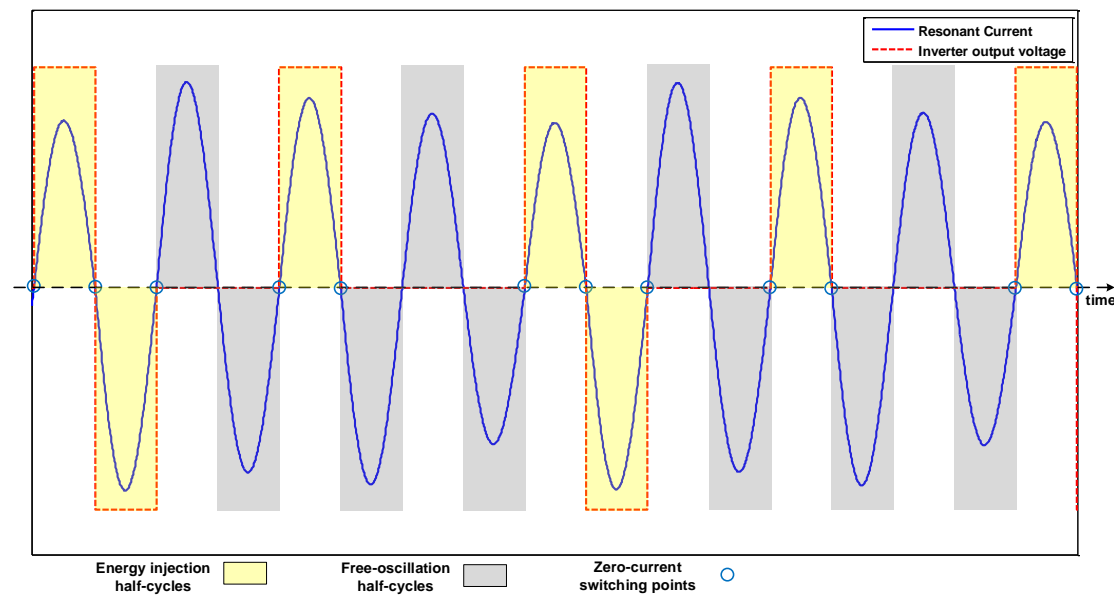

Figure 5.13: The conceptual plot of the primary resonant current and corresponding output voltage of the converter in energy-injection and free-oscillation modes.

an IPT system with a series compensated secondary, the equivalent load resistance reflected to the primary $R_{e q}$ can be calculated as follows [7]:

$$
R_{e q}=\frac{\omega^{2} M^{2}}{R_{L}}
$$

where $\omega$ is the angular resonance frequency and $R_{L}$ is the load at the receiver side. In order to generate an appropriate high-frequency resonant current in the primary 


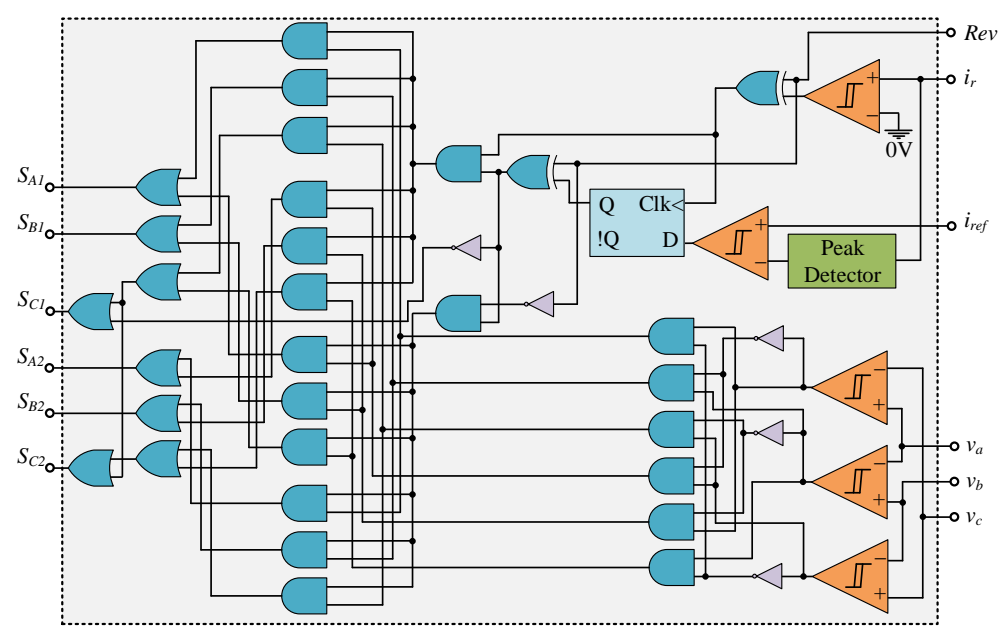

Figure 5.14: The proposed simplified digital power controller for the three-phase AC-AC matrix converter designed based on the switching logic given in (5.28).

of the IPT system and regulate the power transfer rate, a control mechanism should be designed that can achieve the control objectives by switching the converter into different states of operation.

The controller of the three-phase AC-AC converter can be designed based on energy-injection/regeneration and free-oscillation technique. This technique has been used in many studied to regulate the power transfer level in IPT systems $[124,133,139,162,172,183]$. In Fig. 5.13, the conceptual plot of the primary resonant current and corresponding output voltage of the converter of an IPT system in energy-injection and free-oscillation modes for forward power transfer is presented. It can be seen that energy-injection modes increase the resonant current amplitude. In contrast, free-oscillation modes decrease the amplitude of the resonant current. On the other hand, when the converter operates in reverse power transfer, energyinjection modes decrease the resonant current amplitude and free-oscillation modes increase the amplitude of the resonant current. Therefore, in both forward and reverse power transfer modes, the resonant current can be controlled by controlling the energy-injection and free-oscillation modes. In [133], it is shown that the resonant 
current can be calculated as,

$$
i_{r}=\frac{V_{t}-v_{c}(0)}{\omega L} e^{-t / \tau} \sin (\omega t)
$$

where $i_{r}$ is the resonant current, $V_{t}$ is the voltage source applied to the RLC tank, $v_{c}(0)$ is the initial capacitor voltage, $\omega$ is the resonance frequency, $\tau=2 L / R_{e q}$ is the time constant of the RLC tank. In [162], it is shown that in steady-state conditions $v_{c}(0)$ is also a function of $V_{t}$ and RLC circuit parameters. Therefore, based on (5.27), it can be seen that the amplitude of the resonant current can be controlled by controlling $V_{t}$ in each half-cycle.

The energy injection/regeneration technique which is employed in IPT systems controls the applied voltage to the RLC tank $V_{t}$ by switching to different operating modes in order to regulate the power transfer. Based on this technique it can be found that each forward and reverse power transfer modes, the converter has 14 states of operation which are determined based on the direction of the resonant current and the relative order of three-phase input voltages. The forward power transfer mode consists of 12 energy-injection and 2 free-oscillation states which are presented in Table 5.5. The reverse power transfer mode consists of 12 energyregeneration and 2 free-oscillation states which are presented in Table 5.6. According to Tables 5.5 and 5.6 the switching logic for six switches $\left(S_{A 1}, S_{A 2}, S_{B 1}, S_{B 2}, S_{C 1}, S_{C 2}\right)$ 
of the converter can be derived as,

$$
\begin{aligned}
S_{A 1}= & \left(S_{a b} \cdot \overline{S_{c a}} \cdot S_{s g n}+\overline{S_{a b}} \cdot S_{c a} \cdot \overline{S_{s g n}}\right) \cdot S_{i n j} \\
& +\left(S_{a b} \cdot \overline{S_{c a}} \cdot \overline{S_{s g n}}+\overline{S_{a b}} \cdot S_{c a} \cdot S_{s g n}\right) \cdot S_{r e g} \\
S_{A 2}= & \left(\overline{S_{a b}} \cdot S_{c a} \cdot S_{s g n}+S_{a b} \cdot \overline{S_{c a}} \cdot \overline{S_{s g n}}\right) \cdot S_{i n j} \\
& +\left(\overline{S_{a b}} \cdot S_{c a} \cdot \overline{S_{s g n}}+S_{a b} \cdot \overline{S_{c a}} \cdot S_{s g n}\right) \cdot S_{r e g} \\
S_{B 1}= & \left(\overline{S_{a b}} \cdot S_{b c} \cdot S_{s g n}+S_{a b} \cdot \overline{S_{b c}} \cdot \overline{S_{s g n}}\right) \cdot S_{i n j} \\
& +\left(\overline{S_{a b}} \cdot S_{b c} \cdot \overline{S_{s g n}}+S_{a b} \cdot \overline{S_{b c}} \cdot S_{s g n}\right) \cdot S_{r e g} \\
S_{B 2}= & \left(S_{a b} \cdot \overline{S_{b c}} \cdot S_{s g n}+\overline{S_{a b}} \cdot S_{b c} \cdot \overline{S_{s g n}}\right) \cdot S_{i n j} \\
& +\left(S_{a b} \cdot \overline{S_{b c}} \cdot \overline{S_{s g n}}+\overline{S_{a b}} \cdot S_{b c} \cdot S_{s g n}\right) \cdot S_{r e g} \\
S_{C 1}= & \left(S_{c a} \cdot \overline{S_{b c}} \cdot S_{s g n}+\overline{S_{c a}} \cdot S_{b c} \cdot \overline{S_{s g n}}\right) \cdot S_{i n j} \\
+ & \left(S_{c a} \cdot \overline{S_{b c}} \cdot \overline{S_{s g n}}+\overline{S_{c a}} \cdot S_{b c} \cdot S_{s g n}\right) \cdot S_{r e g}+\overline{S_{i n j}} \\
S_{C 2}= & \left(\overline{S_{c a}} \cdot S_{b c} \cdot S_{s g n}+\overline{S_{c a}} \cdot \overline{S_{b c}} \cdot \overline{S_{s g n}}\right) \cdot S_{i n j} \\
+ & \left(\overline{S_{c a}} \cdot S_{b c} \cdot \overline{S_{s g n}}+\overline{S_{c a}} \cdot \overline{S_{b c}} \cdot S_{s g n}\right) \cdot S_{r e g}+\overline{S_{i n j}}
\end{aligned}
$$

where the variables are defined as $S_{a b}: v_{a}>v_{b}, S_{b c}: v_{b}>v_{c}, S_{c a}: v_{c}>v_{a}$, $S_{s g n}: i_{r}>0, S_{i n j}:$ reverse power transfer mode, $S_{r e g}$ : reverse power transfer mode. Using (5.28), a digital controller is designed which is presented in Fig. 5.14. The controller takes three-phase voltages $\left(v_{a}, v_{b}, v_{c}\right)$ and resonant current $\left(i_{r}\right)$ measurements and generates the corresponding switching signals for the converter to regulate the resonant current around the user-defined reference current $\left(i_{r e f}\right)$ and desired power transfer direction $(R e v=0$ for forward power transfer and $R e v=1$ for reverse power transfer). 

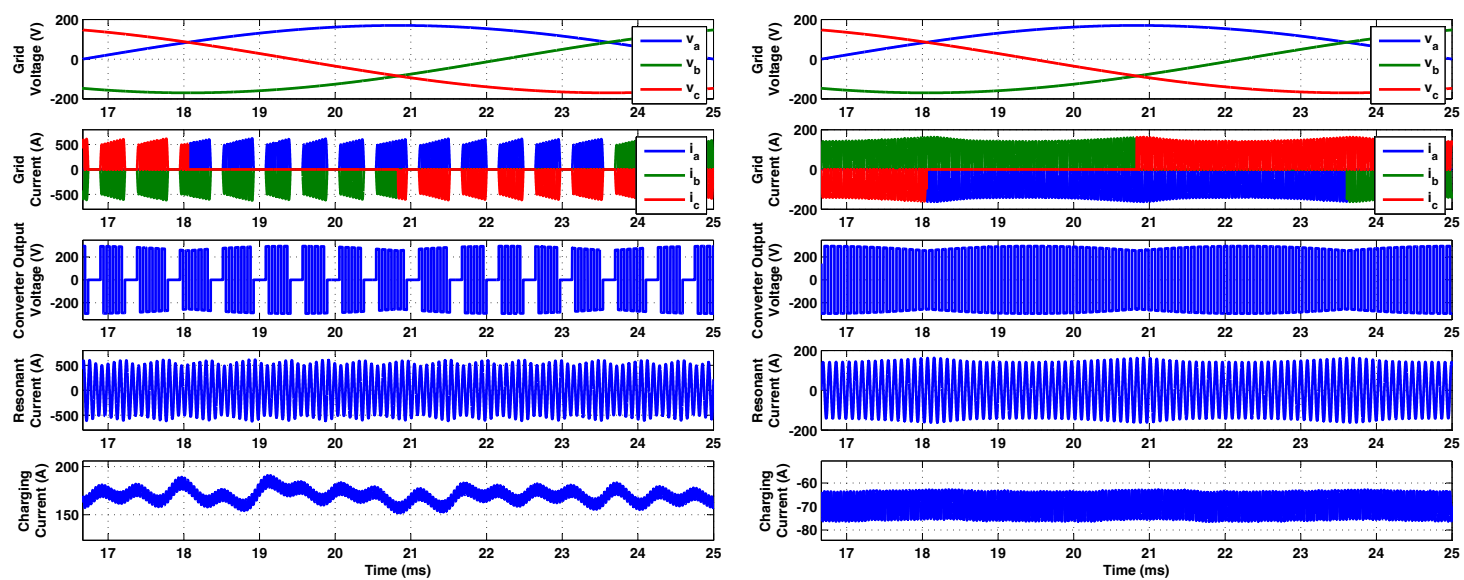

(a)

(b)

Figure 5.15: Simulation results on the proposed AC-AC matrix converter: (a) forward power transfer mode at $68.2 \mathrm{~kW}$ charging power, (b) reverse power transfer mode at $26.4 \mathrm{~kW}$ discharging power,

Table 5.5: Switching states in forward power transfer mode

\begin{tabular}{cccccc}
\hline Mode & $i_{r}>0$ & $v_{a}>v_{b}$ & $v_{b}>v_{c}$ & $v_{c}>v_{a}$ & Switches \\
\hline 1 & 1 & 1 & 1 & 0 & $S_{A 1}, S_{C 2}$ \\
2 & 1 & 1 & 0 & 0 & $S_{A 1}, S_{B 2}$ \\
3 & 1 & 0 & 1 & 0 & $S_{B 1}, S_{C 2}$ \\
4 & 1 & 0 & 1 & 1 & $S_{B 1}, S_{A 2}$ \\
5 & 1 & 0 & 0 & 1 & $S_{C 1}, S_{A 2}$ \\
6 & 1 & 1 & 0 & 1 & $S_{C 1}, S_{B 2}$ \\
7 & 0 & 1 & 1 & 0 & $S_{C 1}, S_{A 2}$ \\
8 & 0 & 1 & 0 & 0 & $S_{B 1}, S_{A 2}$ \\
9 & 0 & 0 & 1 & 0 & $S_{C 1}, S_{B 2}$ \\
10 & 0 & 0 & 1 & 1 & $S_{A 1}, S_{B 2}$ \\
11 & 0 & 0 & 0 & 1 & $S_{A 1}, S_{C 2}$ \\
12 & 0 & 1 & 0 & 1 & $S_{B 1}, S_{C 2}$ \\
13 & 1 & - & - & - & $S_{C 1}, S_{C 2}$ \\
14 & 0 & - & - & - & $S_{C 1}, S_{C 2}$ \\
\hline
\end{tabular}

\subsubsection{Simulation Analysis and Experimental Results}

The proposed converter and its controller connected to an inductive EV charging system are simulated in MATLAB/Simulink. The simulation model consists of transmitter and receiver circuit with $172 \mu \mathrm{H}$ inductance and $120 n \mathrm{~F}$ series-connected compensation capacitors, a $208 V_{L L}$ three-phase voltage source, a secondary AC/DC converter connected to a $22 \mathrm{kWh} 360 \mathrm{~V}$ battery. The simulation results for forward 
Table 5.6: Switching states in reverse power transfer mode

\begin{tabular}{cccccc}
\hline Mode & $i_{r}>0$ & $v_{a}>v_{b}$ & $v_{b}>v_{c}$ & $v_{c}>v_{a}$ & Switches \\
\hline 1 & 1 & 1 & 1 & 0 & $S_{C 1}, S_{A 2}$ \\
2 & 1 & 1 & 0 & 0 & $S_{B 1}, S_{A 2}$ \\
3 & 1 & 0 & 1 & 0 & $S_{C 1}, S_{B 2}$ \\
4 & 1 & 0 & 1 & 1 & $S_{A 1}, S_{B 2}$ \\
5 & 1 & 0 & 0 & 1 & $S_{A 1}, S_{C 2}$ \\
6 & 1 & 1 & 0 & 1 & $S_{B 1}, S_{C 2}$ \\
7 & 0 & 1 & 1 & 0 & $S_{A 1}, S_{C 2}$ \\
8 & 0 & 1 & 0 & 0 & $S_{A 1}, S_{B 2}$ \\
9 & 0 & 0 & 1 & 0 & $S_{B 1}, S_{C 2}$ \\
10 & 0 & 0 & 1 & 1 & $S_{B 1}, S_{A 2}$ \\
11 & 0 & 0 & 0 & 1 & $S_{C 1}, S_{A 2}$ \\
12 & 0 & 1 & 0 & 1 & $S_{C 1}, S_{B 2}$ \\
13 & 1 & - & - & - & $S_{C 1}, S_{C 2}$ \\
14 & 0 & - & - & - & $S_{C 1}, S_{C 2}$ \\
\hline
\end{tabular}

power transfer at $64.2 \mathrm{~kW}$ and reverse power transfer at $26.4 \mathrm{~kW}$ are presented in Figs. 5.15(a) and (b) respectively. These results verify that the converter can effectively establish a bidirectional connection between the IPT system and the power grid at different power transfer levels. This figure also shows that the converter generates a high-frequency resonant current in the IPT system which is regulated by controlling the energy injection/regeneration level. The plots of the three-phase grid voltage and unfiltered grid current show that the converter switches to different phases whenever grid voltages cross each other. This ensures that the maximum power transfer is always achieved.

The proposed three-phase AC-AC matrix converter along with its controller is implemented experimentally and is tested on case study IPT system as shown in Fig. 5.16. The controller is implemented according to the design which is presented in Fig. 5.14. The controller is composed of differential comparators, peak detector and an FPGA board (Xilinx Spartan 6) to perform the logical operations. The IPT system test-bed is composed of transmitter and receiver power pads, each having $172 \mu \mathrm{H}$ inductance and a series connected $120 n \mathrm{~F}$ compensation capacitor thereby, resulting in a $35 \mathrm{kHz}$ resonance frequency. A three-phase variable power supply is 


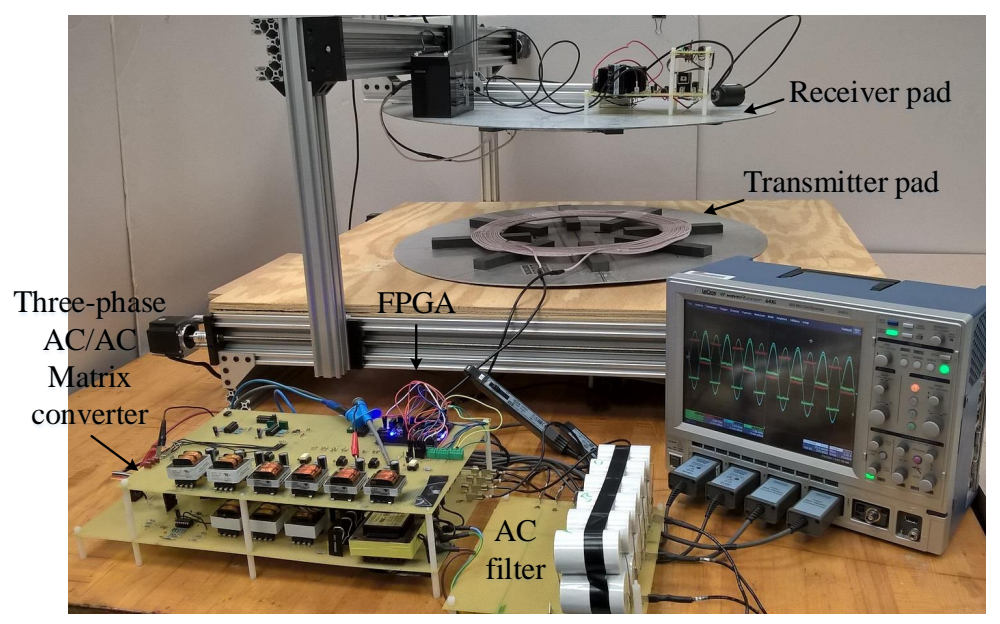

Figure 5.16: The IPT system setup used for experimental analysis.

used as the grid connection. In order to validate the performance of the converter, experiments are carried out at low-voltage grid connection at low-power level. In Fig. 5.15, $S_{A 1}$ and $S_{B 2}$ switching signals (according to Fig. 5.12) at $35 \mathrm{~W}$ forward power transfer rate are presented. This figure shows that the converter achieves zero-current switching operation and effectively generates high-frequency resonant current from a low-frequency AC main.

The harmonic content of the grid current can be effectively mitigated by regular LC power filters. Also, high-order power filters similar to the single-phase topology presented in $[186,187]$ can be employed to further improve the power quality of the proposed converter.

\section{$5.3 \quad$ Summary}

In this chapter, direct three-phase single-stage ac-ac converters for inductive power transfer (IPT) systems are introduced. The proposed converters have different control modes, which can be used to regulate the resonant current, resonant voltage and output power around user-defined reference values. The energy injection and 


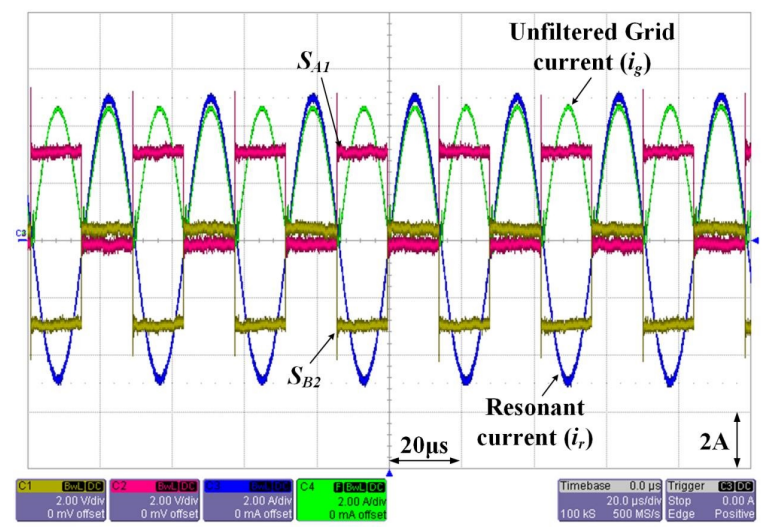

Figure 5.17: Experimental results on the proposed three-phase matrix converter pro-to-type: resonant current $\left(i_{r}\right)$, and unfiltered phase A current $\left(i_{a}\right)$, switching signals $S_{A 1}, S_{A 2}$.

free oscillation control strategy provides a simple and effective method, which makes it suitable for the control of IPT systems. Key benefits of the proposed converter are the minimized number of switches, soft-switching operation, and elimination of short-life electrolytic capacitors. These features will lead to high-efficiency converters with high reliability and extended lifetime. Specifically, with the use of reverse blocking switches, a direct AC-AC matrix converter is developed and built only with seven switches. The simulation results and experimental studies show that the proposed converters can maintain the resonant current or the output power around the user-defined reference values. This feature makes the converters suitable for dynamic IPT systems, where the system has inherent variations. The simulation and experimental results on the proposed converters with power controller show that the converters can effectively establish a bidirectional power transfer connection between the IPT system and the power grid at a desired power level making it suitable for forming contactless G2V and V2G connections. The converter achieves zero-current switching (soft-switching) and can track the resonance frequency in dynamically varying IPT systems which both together ensure the high efficiency operation of the system. The soft-switching operations significantly reduce the complexity of the 
controller design by eliminating the need for multi-stage commutation processes for bidirectional switches. Moreover, the controller is designed based on a simplified switching logic which can be easily implemented on FPGA boards without the need for DSP platforms. 
CHAPTER 6

\section{APPLICATIONS OF SELF-TUNING CONTROLLERS IN INDUCTIVE ELECTRIC VEHICLE CHARGING SYSTEMS}

The self-tuning controllers which are introduced in Chapter 3.1 can find many applications in inductive EV charging system. In this chapter, a few applications that utilize the power transfer control along with resonance frequency tracking capabilities of the developed controllers are introduced. The work presented in this chapter is published in $[188,189]$.

\subsection{Sensorless Conductive Foreign Object Detection for In- ductive Electric Vehicle Charging Systems Based on Resonance Frequency Deviation}

In this section, a sensorless conductive foreign object detection (FOD) method for inductive power transfer (IPT) systems based on resonance frequency deviation monitoring is proposed. The proposed detection method is based on the fact that the proximity of conductive objects to the transmitter area will affect the resonant characteristics of the system, including resonance frequency. Thereby, the conductive object detection is achieved by constantly monitoring the resonance frequency deviation with respect to its nominal value. Self-tuning controllers for IPT systems are utilized to synchronize the switching power converters with the resonance current in order for the system to operate at the resonance frequency. The proposed method is specifically designed for standby mode FOD in which detection is performed at low power before the entrance of the vehicle to the charging station. Theoretical analysis of the proposed method based on circuit theory and 3D finite element analysis (FEA) is presented. Also, the proposed method is implemented in 
a case study IPT system and its effectiveness is verified experimentally by detecting different conductive objects. The results show that the proposed method is fast, sensitive, effective, and is capable of detecting conductive objects as small as a 5cent coin in standby mode. Based on the proposed FOD, two detection modes are proposed: standby detection mode and online detection mode. In standby mode, the charging area is continuously examined for any conductive foreign object without the presence of the vehicle at low power. On the other hand, in online detection mode, FOD is performed along with the charging process at charging power levels.

Theoretical analysis of the proposed method based on circuit theory and 3D finite element analysis (FEA) is presented. The proposed method is implemented on a case study IPT system and its effectiveness is verified experimentally in detection of different conductive objects. The results show that the proposed method is very fast, sensitive and effective and is capable of detecting conductive objects as small as a 5-cent coin.

\subsubsection{Effects of Conductive Foreign Objects on IPT Systems}

When a conductive object is placed in the power transfer area in an IPT system, due to high-frequency magnetic fields generated by the coils, the object acts as

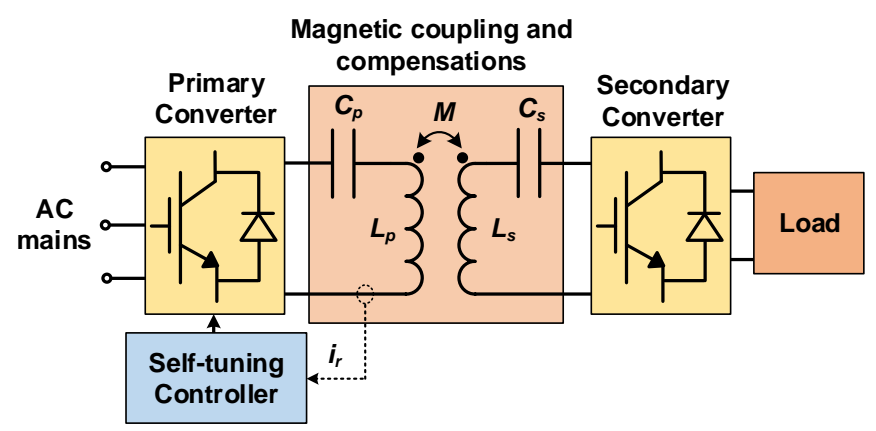

Figure 6.1: Typical IPT system with a primary self-tuning controller. 


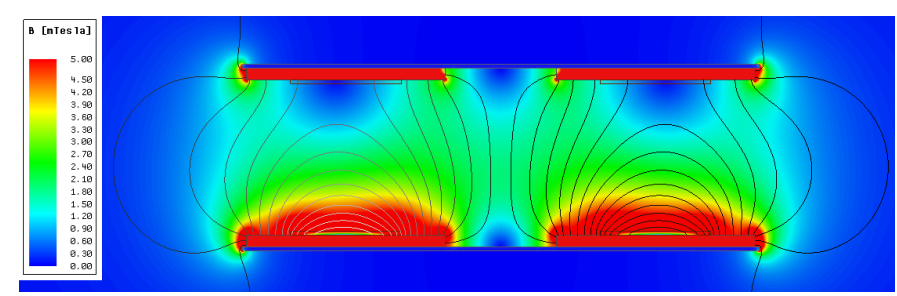

(a)

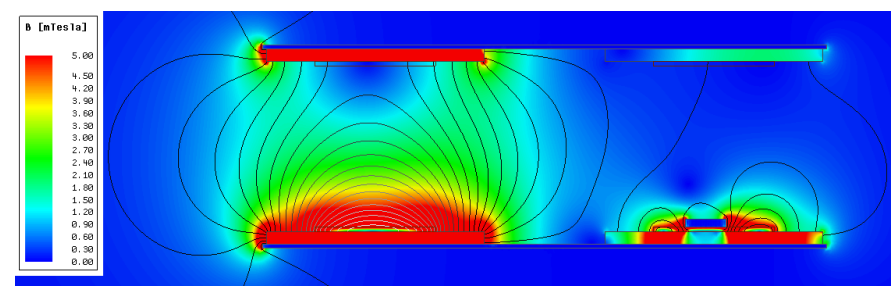

(b)

Figure 6.2: The magnetic flux density inside the case study IPT system: (a) normal operation, (b) a conductive object is placed on the primary coil.

an electromagnetic shield and as a result, it dramatically affects the distribution of magnetic flux lines around it. Consequently, characteristics of the magnetic couplers change, including the self-inductances of the primary and secondary $\left(L_{p}\right.$ and $\left.L_{s}\right)$ and the mutual-inductance between primary and secondary $(M)$. The changes on the inductances significantly depend on the position of the conductive object relative to the magnetic couplers, size, shape, and material type of the object. Figure 6.2(a) shows the magnetic flux lines of a sample IPT system with circular power pads, which are calculated using 2D finite element analysis (FEA). Figure 6.2(b) shows the distorted magnetic flux lines with the presence of a conductive object in the power transfer area.

\subsubsection{Conductive Foreign Object Detection Method}

The effects of a conductive foreign object on the resonance frequency of an IPT systems can be captured by analyzing its effects on self and mutual inductances $\left(L_{p}\right.$, 
$L_{s}$ and $\left.M\right)$ of the system. The equivalent impedance of a series-series compensated IPT system which is shown in Fig. 6.1 can be written as follows:

$$
Z=R_{p}+\frac{1}{s C_{p}}+s L_{p}+Z_{r}
$$

where, $R_{p}$ is the primary resistance, $C_{p}$ is the primary compensation capacitor, $Z_{r}$ is the reflected impedance of the secondary into primary, which can be calculated as follows:

$$
Z_{r}=-\frac{s^{2} M^{2}}{\frac{1}{s C_{s}}+s L_{s}+R_{s}}
$$

where $R_{s}$ is the equivalent resistance of the secondary which includes the load resistance, $C_{s}$ is the secondary compensation capacitor. By considering primary current as the output and primary voltage as the input of the system, the transfer function of the system can be written as follows:

$$
H(s)=\frac{i_{p}}{v_{p}}=\frac{1}{Z}
$$

Using (6.1) and (6.2), the transfer function given in (6.3) can be expressed as follows:

$$
\begin{aligned}
H(s)= & \frac{s C_{p}\left(1+s^{2} C_{s} L_{s}+s C_{s} R_{s}\right)}{\left[C_{p} C_{s}\left(L_{p} L_{s}-M^{2}\right) s^{4}+C_{p} C_{s}\left(L_{s} R_{p}+L_{p} R_{s}\right) s^{3}+\right.} \\
& \left.\left(C_{p} L_{p}+C_{s} L_{s}+C_{p} C_{s} R_{p} R_{s}\right) s^{2}+\left(C_{p} R_{p}+C_{s} R_{s}\right) s+1\right]
\end{aligned}
$$

The derived transfer function has four poles that are comprised of two pairs of complex conjugate numbers. The poles which are closer to the imaginary axis in the complex plane are defined as the dominant poles and their imaginary parts are defined as the resonance frequency of the IPT system (in $\mathrm{rad} / \mathrm{s}$ ). The presence of a conductive foreign object close to the charging area affects the values of the inductances $L_{p}, L_{s}$ and $M$. As a result the resonance frequency gets affected, as these parameters form the coefficients of the denominator of (6.4). Therefore, the 


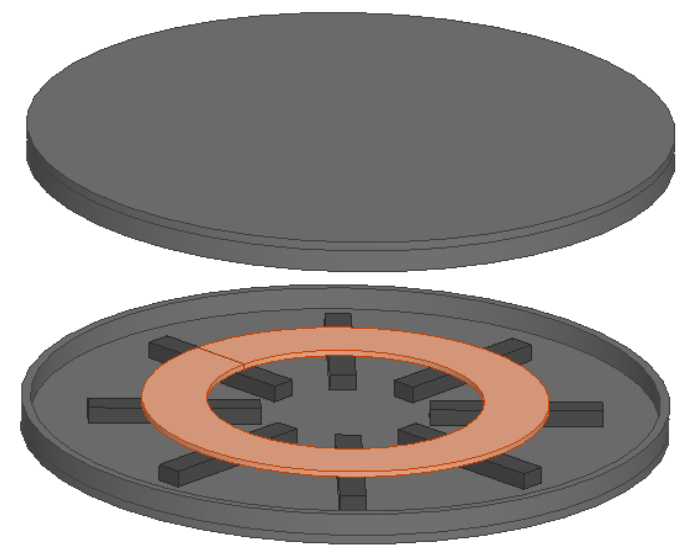

Figure 6.3: 3D model of the case study IPT system.

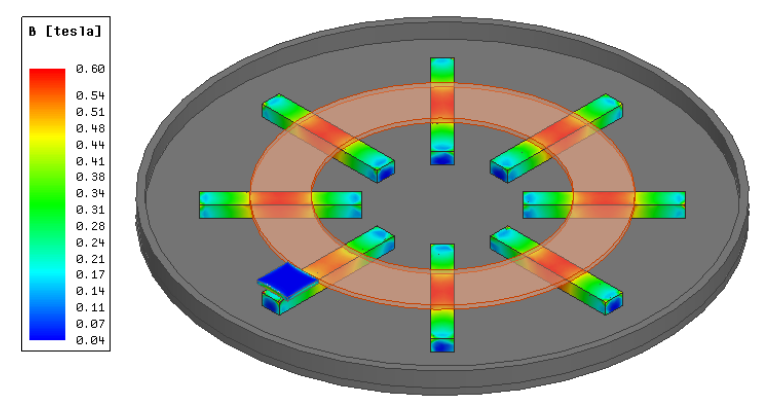

Figure 6.4: Flux density in the circular power pad.

presence of a conductive foreign object can be detected by measuring the resonance frequency.

To demonstrate how an IPT system is affected by a conductive foreign object, a sample IPT system is analyzed using 3D finite element analysis (FEA). The 3D model of the sample IPT system, consisting of two circular power pads is shown in Fig. 6.3. FEA is used to calculate $L_{p}, L_{s}$ and $M$ with conductive foreign object located at different positions relative to the center of the charging pad on top of the primary pad. Figs. 6.4 and 6.5 show the flux density inside the ferrite bars and surface loss density on the external object respectively.

Figure 6.6 shows the self and mutual inductances as a function of the location of the foreign object relative to the center of the primary pad. In Fig. 6.7 the 


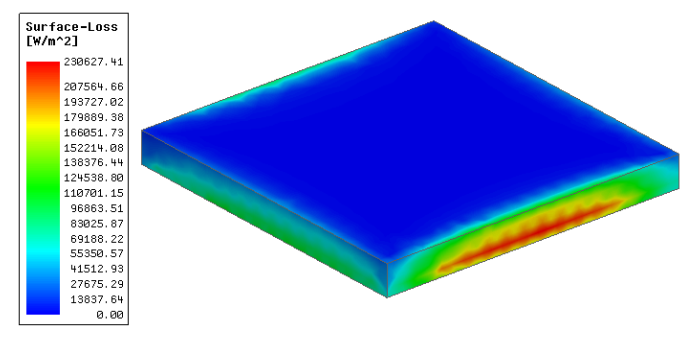

Figure 6.5: Surface loss density on the sample foreign object.

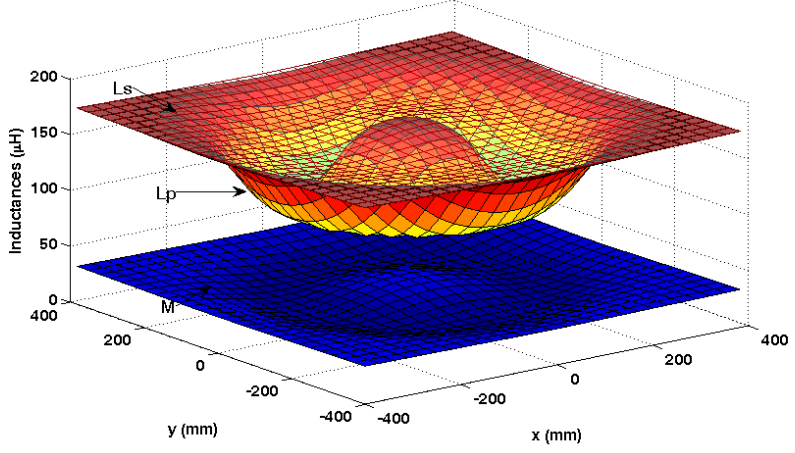

Figure 6.6: Primary self-inductance $\left(L_{p}\right)$, secondary self-inductance $\left(L_{s}\right)$ and mutual-inductance $(M)$ versus the location of the conductive object relative to the center of the transmitter.

corresponding resonance frequency of the IPT system, which is obtained by calculating the dominant poles of the transfer function (6.4) at each point is shown. This figure shows that the frequency deviation increases as the conductive object is placed closer to the coils, and it is maximum on the coils. This figure also shows that if the object is placed exactly in the center of the pad, the frequency deviation would be almost zero. This can be justified by the fact that due to the symmetry of the magnetic structure, the flux density in the center of the pads is canceled and consequently the effect of the conductive foreign object on the flux lines would be negligible.

As it is shown in Fig. 6.7, the presence of conductive objects always tends to increase the resonance frequency while the presence of vehicle always tends to 


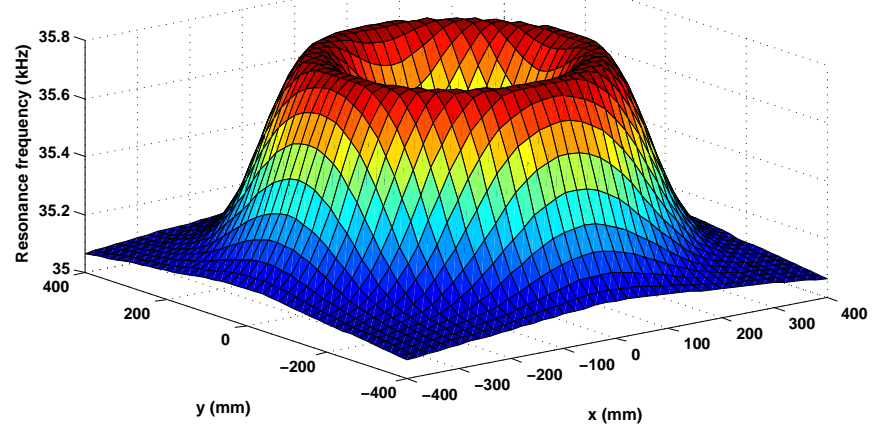

Figure 6.7: Resonance frequency of the IPT system versus the location of the conductive object respect to the center of the transmitter pad, calculated using 3D FEA.

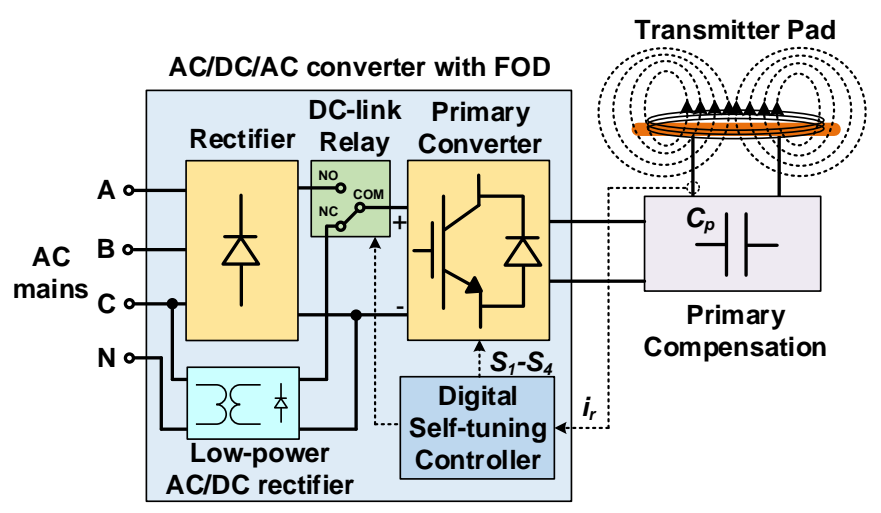

Figure 6.8: The proposed primary converter system with self-tuning controller and FOD and a low-power DC source for initial (standby) detection.

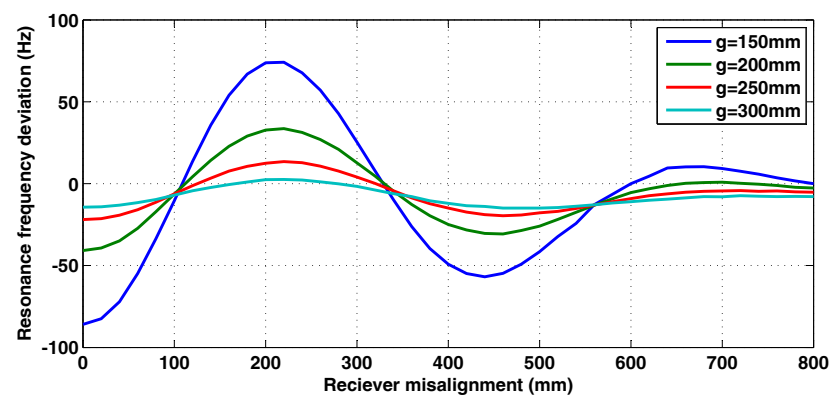

Figure 6.9: Resonance frequency deviation of the IPT system versus the location of the receiver pad relative to the center of the transmitter pad. 
decrease the resonance frequency. In addition, the resonance frequency further drops once the receiver is energized (starts the charging process). In other words the resonance frequency can be expressed as follows:

$$
\begin{gathered}
f_{r e f}=f_{r 0}-\Delta f_{V}-\Delta f_{L} \\
\Delta f_{O}=f_{r}-f_{r e f}
\end{gathered}
$$

where $f_{r e f}$ is the reference frequency, $f_{r 0}$ is the normal resonance frequency of the primary pad without the presence of the vehicle, $\Delta f_{V}, \Delta f_{L}$ and $\Delta f_{O}$ are resonance frequency deviations caused by vehicle alignment, charging process and conductive foreign object respectively.

\subsubsection{Initial Foreign Object Detection at Low Power}

In an inductive EV charging station, initial conductive foreign object detection at very low power can be applied to the transmitter coil to continuously monitor the charging area for any conductive object, prior to the presence of the EV and the charging process initialization. In this case, $\Delta f_{V}=\Delta f_{L}=0$ and therefore based on (6.6), conductive objects can be detected. In Fig. 6.8, the primary converter with a digital self-tuning controller along with an FOD mechanism is shown. The DC-link can be switched between the main three-phase rectifier and a low-power low-voltage rectifier. The low-power DC-source enables the system to perform the initial FOD in standby mode.

\subsubsection{System Losses in Initial Foreign Object Detection Mode}

The losses associated with the initial FOD mode can be calculated as follows [162]:

$$
P=\frac{2 \omega V_{d c}^{2} \tau^{2}\left(1+e^{-\pi / \tau \omega}\right)}{\pi L\left(1+\tau^{2} \omega^{2}\right)\left(1-e^{-\pi / \tau \omega}\right)}
$$


where $\omega=\sqrt{\omega_{0}^{2}-1 / \tau^{2}}$ is the damped resonance frequency, $\omega_{0}=1 / \sqrt{L C}$ is the natural resonance frequency, $C$ is the primary compensation capacitor, $\tau=2 L / r_{c}$ is the damping time constant, $L$ is the self-inductance of the transmitter, $r_{c}$ is the resistance of the transmitter coil, and $V_{d c}$ is the DC-link voltage.

\subsubsection{Online Foreign Object Detection Along With Charging Process}

The proposed FOD can be used during the charging process. However, vehicle variations can also affect the magnetic characteristics of the IPT system including resonance frequency. In Fig. 6.9, the resonance frequency deviations in the the case study inductive charging system, due to vehicle variations, as a function of vehicle position respect to the center of the transmitter pad is presented. This figure shows that the maximum resonance frequency deviation due to vehicle variations is about $74 \mathrm{~Hz}$ (with $150 \mathrm{~mm}$ air gap). As the vehicle can have variations during the charging process, it would be impossible to discriminate the intrusion of small foreign objects (such as 5-cent coin and 10d nail, which are presented in Section 6.1.3) with vehicle variations based on resonance frequency deviation. However, larger objects can be easily distinguished, as their effect on resonance frequency would be much higher than the effect of vehicle variations. For instance, a beverage can causes frequency deviations as high as $660 \mathrm{~Hz}$ (Section 6.1.3).

\subsubsection{Foreign Object Detection Speed}

The frequency of the resonant current can be measured by counting the number of cycles within a specific sampling time period. This is achieved by using the current measurement signal as a clock source for a timer/counter. The number of cycles 


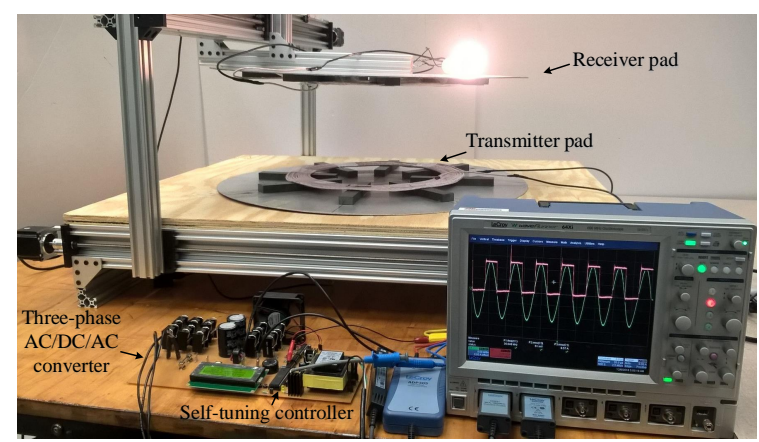

Figure 6.10: The case study IPT system with a self-tuning controller.

which can be counted in the sampling time period can be calculated as follows:

$$
N_{c y c}=\frac{T_{s}}{t_{c y c}}
$$

where $N_{c y c}$ is the number of counted cycles in the sampling time period $T_{s}, f_{s}=1 / T_{s}$ is the sampling frequency, $t_{c y c}=1 / f_{r}$ is the time period of the resonant current.

$$
N_{c y c}=f_{r} T_{s}
$$

Based on (6.9), the minimum frequency deviation which can be detected would be as follows:

$$
d f_{\text {min }}=\frac{f_{r}}{N_{c y c}}=\frac{1}{T_{s}}=f_{s}
$$

Equation (6.10) shows that the minimum detectable frequency deviation equals to the sampling frequency. For example, if with $1 \mathrm{~ms}$ and $10 \mathrm{~ms}$ sampling times, the frequency deviations more than $1 \mathrm{kHz}$ and $100 \mathrm{~Hz}$ would be detectable respectively. This shows that the sensitivity of the detection method increases as the sampling time decreases. In other words, the accuracy of the FOD is compromised as the detection speed is decreased. 
Table 6.1: Specifications of the case study system.

\begin{tabular}{cc}
\hline Parameter & Value \\
\hline Air gap & $200 \mathrm{~mm}$ \\
Pad diameter & $700 \mathrm{~mm}$ \\
Self-inductances $\left(L_{p}, L_{s}\right)$ & $172 \mu \mathrm{H}$ \\
Compensation capacitors $\left(C_{p}, C_{s}\right)$ & $120 \mathrm{nF}$ \\
Nominal resonance frequency $\left(f_{r}\right)$ & $35 \mathrm{kHz}$ \\
Grid voltage $\left(V_{a c}\right)$ & $120 \mathrm{~V}$ \\
Grid frequency $\left(f_{a c}\right)$ & $60 \mathrm{~Hz}$ \\
\hline
\end{tabular}

\subsubsection{Experimental Analysis}

The proposed conductive FOD method is experimentally tested on a case study IPT system for different conductive objects with different shapes, sizes, and positions including beverage cans and coins. The case study IPT system shown in Fig. 6.10 is composed of two circular power pads, compensation capacitors, a primary three-phase AC/DC/AC converter. The specifications of the case study IPT system is presented in Table 6.1. A self-tuning controller is used to generate the switching signals that are synchronized with the resonance frequency for the primary converter. The controller also measures the frequency of the resonant current. The controller can detect any deviation from nominal resonance frequency and disables the switching signals if the deviation exceeds a threshold user-defined value.

The experiments were carried out by placing the objects at different positions relative to the transmitter and receiver power pads and the frequency deviations were measured. The experimental test results along with 3D FEA simulation results and corresponding FOD speeds are presented in Fig. 6.11. This figure shows that the simulation results conform with experimental ones with a small discrepancy. The maximum frequency deviations caused by the presence of the coin and the beverage can are $24 \mathrm{~Hz}$ and $660 \mathrm{~Hz}$ respectively. The detection area for an object with a specific sensitivity of detection frequency deviation (speed) is defined as the percentage of the primary charging pad area in which the object can be detected. 


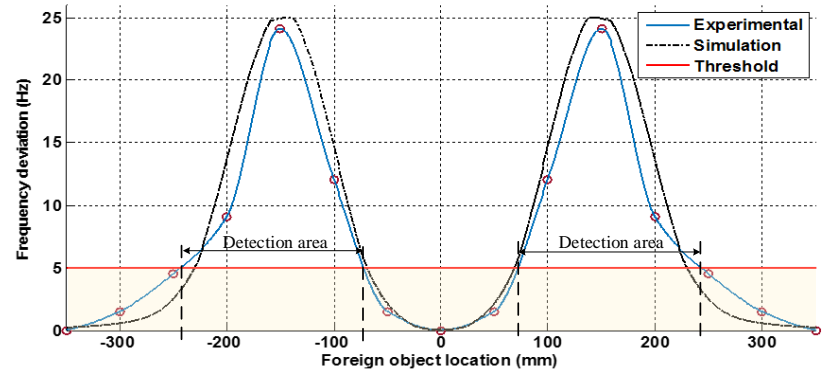

(a)

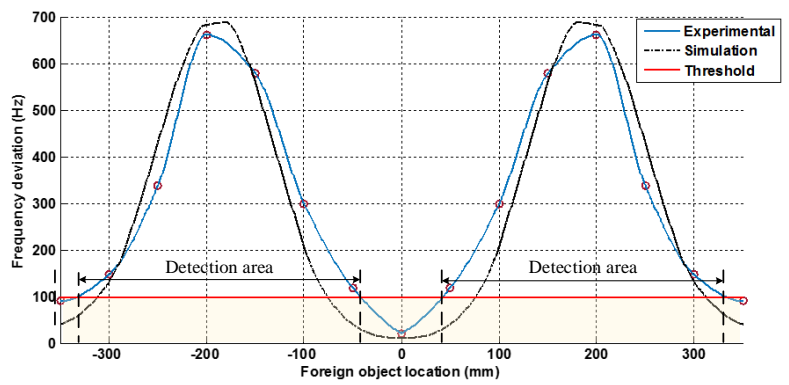

(b)

Figure 6.11: Experimental test results on different foreign objects: (a) 5-cent coin with $200 \mathrm{~ms}$ detection speed and 49\% detection area, (b) beverage can with $10 \mathrm{~ms}$ detection speed and $82 \%$ detection area.

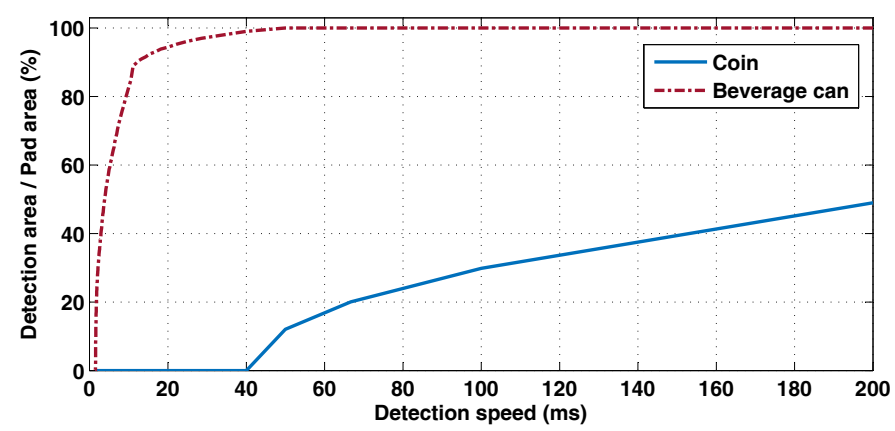

Figure 6.12: Detection area versus detection speed calculated based on the experimental test results on the case study IPT system.

As it can be seen in Fig. 6.11, a beverage can is detected 10 times faster than a coin with much larger detection area.

Fig. 6.12 shows the detection area (in percentage of the pad area) as function of detection speed (in ms) for different objects, which is calculated based on ex- 
perimental results presented in Fig. 6.11. This figure shows that with the same detection area, larger objects can be detected at much higher speeds.

\subsection{Sensorless Electric Vehicle Detection in Inductive Charg- ing Stations Using Self-Tuning Controllers}

In this section, a sensorless electric vehicle (EV) detection mechanism for inductive charging stations is proposed. The proposed method is based on the detection of small resonance frequency deviations from the nominal resonance frequency which are due to the presence of the vehicle. Self-tuning controllers are utilized for fast and accurate resonance frequency tracking in inductive charging systems. The proposed method uses the transmitter magnetic structure of inductive charging systems at very low power for vehicle detection and thereby, eliminates the need for sensorbased detection mechanisms. The proposed method is simulated using 2D and 3D finite element analysis (FEA) and the results are presented. The results show that in a typical inductive charging station, vehicles can be detected within 1.5 meters from the transmitter pad.

Since EVs and specifically their chassis and the wireless power receiver magnetic structure mounted under the EV are composed of various structural metal components, they can have an impact on the magnetic fields generated by the transmitter coils. As a result, the magnetic characteristics of an inductive charging system are changed due to the proximity of EVs. The proposed vehicle proximity detection method is based on the detection of small resonance frequency deviations from the nominal resonance frequency, which are due to the presence of the vehicle in the charging area. Self-tuning controllers are utilized for fast and accurate resonance frequency tracking. The proposed method uses the primary magnetic structure in 
inductive charging systems for proximity detection and thereby eliminates the need for sensor-based detection methods. The proposed method is simulated using $2 \mathrm{D}$ and 3D finite element analysis (FEA) and the results are presented.

\subsubsection{Proposed Vehicle Detection Method}

The operating resonance frequency is one of the key parameters of any IPT system. SAE TIR J2954 standard requires inductive charging systems for light-duty EVs to have an operating frequency within $81.38 \mathrm{kHz}$ to $90 \mathrm{kHz}$ range [90]. The resonance frequency of an IPT system depends on the compensation topology and the selfinductance of the transmitter and receiver pads. Since compensation topology in an IPT system is fixed, any change in the resonance frequency would be due to a change in the self-inductance of the system.

Since EVs and specifically their chassis and the wireless power receiver magnetic structure mounted under the EV are composed of various structural metal components, they can have an impact on the magnetic fields generated by the transmitter coil. As a result, the self-inductance of the transmitter changes due to the proximity of EVs. Thereby, the resonance frequency of the IPT system deviates from its

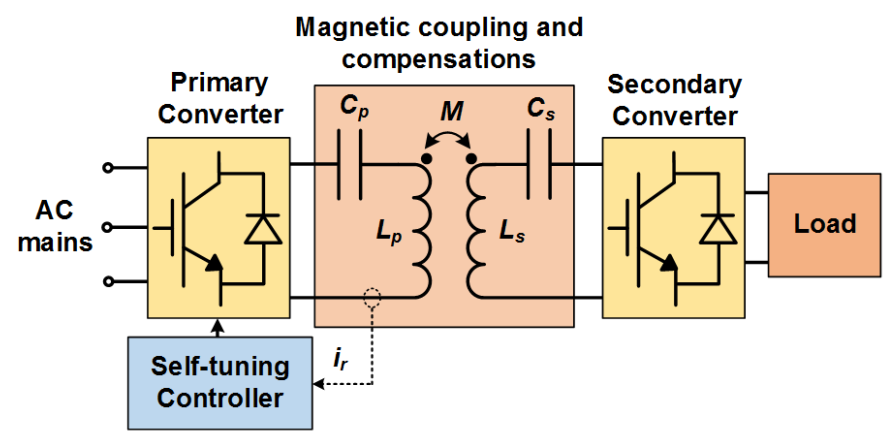

Figure 6.13: A typical inductive charging system with a self-tuning controller in the primary side. 


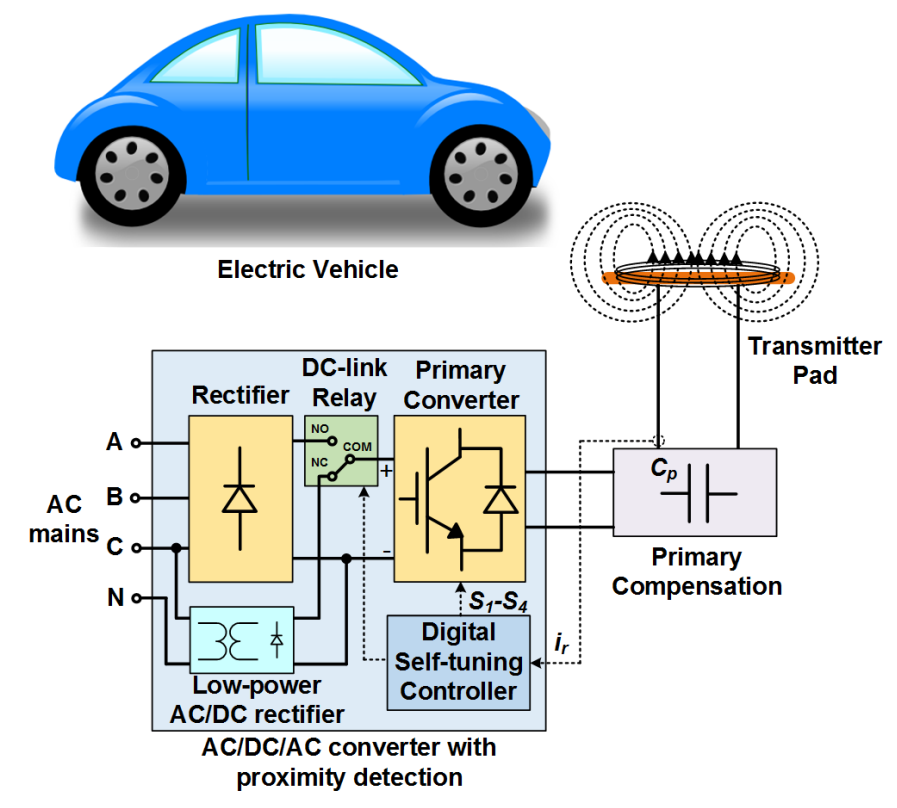

Figure 6.14: The proposed proximity detection system based for inductive electric vehicle charging systems.

nominal value. The proposed vehicle proximity detection method is based on the capturing the small resonance frequency deviations which caused by the presence of the EV. Self-tuning controllers are utilized for fast and accurate resonance frequency tracking. The proposed method uses the same inductive charging system for proximity detection and thereby eliminates the need for sensor-based detection methods.

In Fig. 6.14, an inductive charging system with the proposed vehicle proximity detection system is presented. As it is shown, the DC-link of the primary converter can be connected either to the main three-phase rectifier or the low-power lowvoltage rectifier. The DC-link of the converter is by default connected to the lowvoltage rectifier for vehicle proximity detection. Once the vehicle is perfectly aligned with the transmitter, the DC-link of the converter is switched to the main rectifier and thereby the inductive charging can be initiated. 


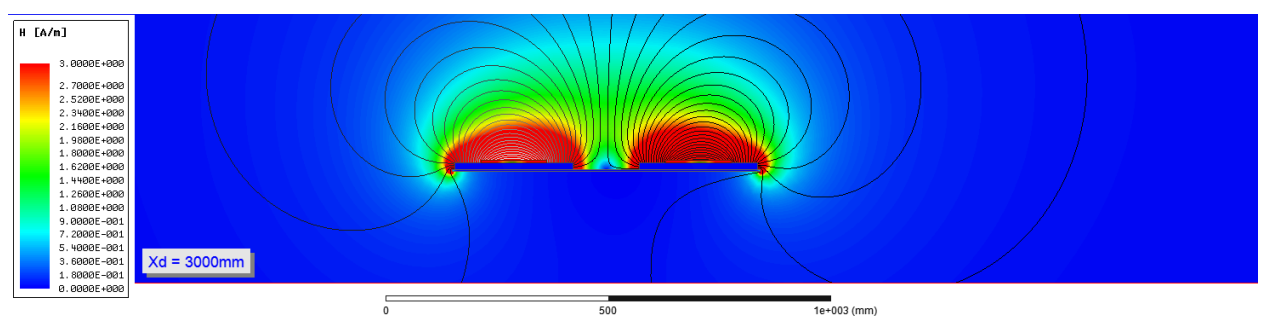

(a)

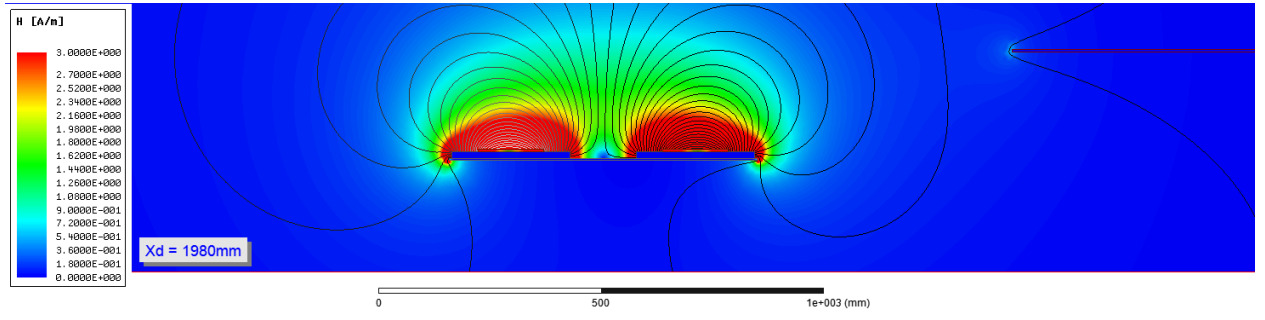

(b)

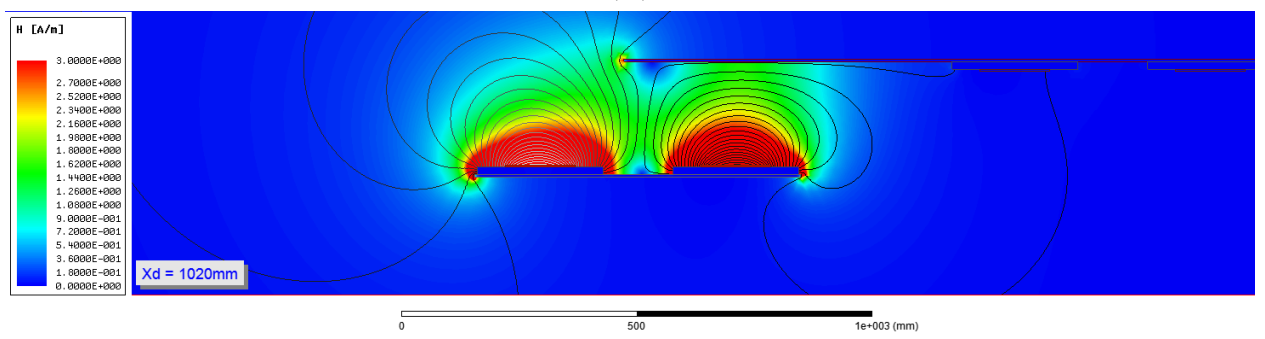

(c)

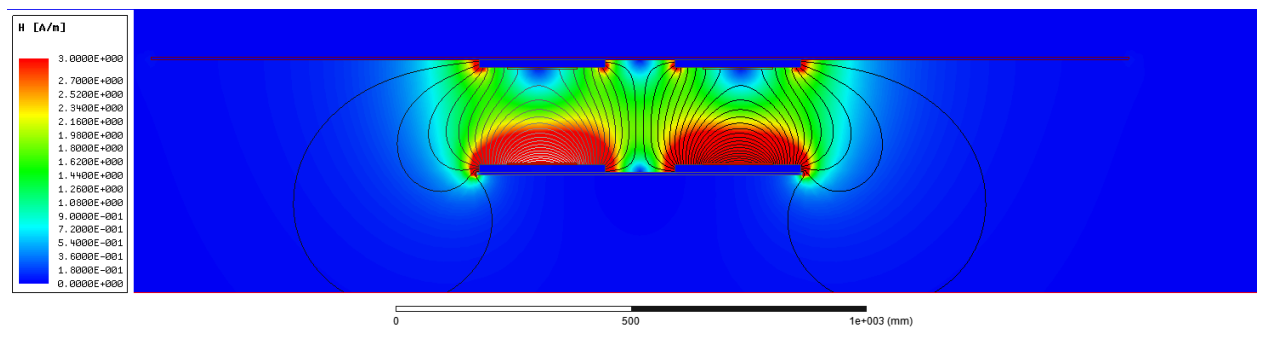

(d)

Figure 6.15: Magnetic flux lines and field intensity of the wireless charging system at different transmitter and receiver pad center-to-center distances: (a) 3 meters, (b) 2 meters, (c) 1 meter, (d) 0 meter.

\subsubsection{Simulation Analysis}

In order to evaluate and verify the proposed detection method, a physics-based model is required to analyze the effect of an EV on the magnetic characteristics of inductive charging systems. Physics-based models based on 3D finite element anal- 
Table 6.2: Specifications of the case study inductive charging system.

\begin{tabular}{ll}
\hline \hline Parameter & Description \\
\hline shielding plate diameter & $700 \mathrm{~mm}$ \\
internal diameter of the coil & $350 \mathrm{~mm}$ \\
width of the coil & $150 \mathrm{~mm}$ \\
number of turns & 15 \\
internal diameter of the ferrite ends & $100 \mathrm{~mm}$ \\
ferrite bar length & $270 \mathrm{~mm}$ \\
ferrite bar width & $28 \mathrm{~mm}$ \\
ferrite bar thickness & $16 \mathrm{~mm}$ \\
no. of ferrite bars & 8 \\
\hline
\end{tabular}

ysis (FEA) can be developed to calculate the quasi-static magnetic field quantities. The FEA model includes the magnetic structures of the IPT system as well as the EV. An inductive charging system comprising of two circular power pads as the transmitter and receiver magnetic structures is investigated as a case study. The specifications of the case study IPT system is presented in TABLE 6.2. In Fig. 6.15, the magnetic flux lines and field intensity at different transmitter and receiver pad center-to-center distances are presented. The vehicle is modeled as a chassis that the receiver pad is mounted under it. It can be seen, as the vehicle gets closer to the transmitter pad, its effect on the magnetic flux lines increases. However, as it continues to completely get aligned with the transmitter, the distortion in the flux lines will be reduced.

In Fig. 6.16, the self-inductance of the primary which is calculated using 3D FEA model and the corresponding resonance frequency of the IPT system as functions of vehicle alignment (perfect alignment at vehicle alignment=0) respect to the primary power pad are presented. This figure shows that the presence of the vehicle within 1.5 meters of the perfect alignment causes the self-inductance to decrease and thereby, the resonance frequency increases. Once the vehicle is perfectly aligned, the selfinductance of the system as well as the resonance frequency reach to their initial values. Based on Fig. 6.16 it can be implied that the proximity of the vehicle can 


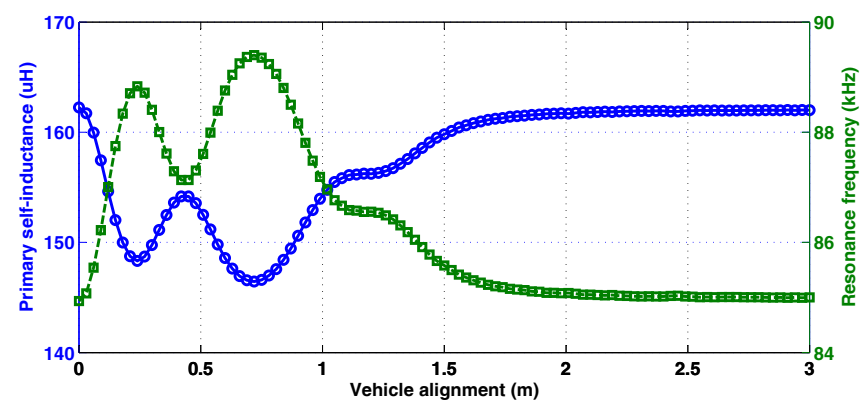

Figure 6.16: Self-inductance of the primary and corresponding resonance frequency of the IPT system as function of vehicle alignment respect to the primary power pad.

be easily detected by tracking the resonance frequency and detecting its deviations from the nominal operating frequency. As the vehicle aligns with the primary power pad, the resonance frequency returns to its initial value, which indicates that the inductive charging can be initiated. 


\section{CHAPTER 7 \\ CONCLUSIONS AND FUTURE WORK}

Inductive Power Transfer (IPT) systems based on the resonant magnetic induction constitute a new technology which enables power transmission between two systems without physical contacts. Owing to its superior robustness, reliability and safety in comparison with the existing methods, they are applied widely as bio-medical implants, material handling, transportation systems. Over the recent years, the IPT-based charging systems for contactless Electric Vehicles (EVs) has been on the rise, broadly categorized as static, and dynamic or in-motion charging modes.

Presenting themselves as a formidable alternative to the traditional wired charging in EV charging stations, static EV charging systems provide more safety and convenience to the users of EVs. At the same time, a growing interest has emerged in the dynamic EV charging systems, and it is now regarded as one of the technological cornerstones that can revolutionize the world of transportation systems. The contactless EV charging systems are equipped with bidirectional power flow capabilities to enable Grid-to-Vehicle (G2V) and Vehicle-to-Grid (V2G) connections. Improving the resiliency of the future infrastructure of smart grid is touted to be one of the major benefits of V2G connections, since they can afford to support the grid under extreme conditions, or through peak shaving.

In Chapter 2, a generalized multi-objective optimization method which provides a holistic solution for obtaining the magnetic design parameters of inductive EV charging systems has been introduced. Using the proposed method, the magnetic structures can be effectively designed by prioritizing the objectives. The design objectives include power transfer efficiency, misalignment tolerance, power density and cost efficiency. The proposed method employs a multi-physics model that incorporates both electromagnetic and thermal models of the inductive charging system 
for precise calculation of power losses, stray field and hot-spot temperature. The experimental results on a $3.7 \mathrm{~kW}$ conform to the theoretical calculations. Although the proposed method is computationally intensive, using parallel processing the optimization time can be significantly reduced. The application of the proposed physics-based design methodology in other inductive charging topologies will be investigated as future works.

In Chapter 3, design and development of self-tuning soft-switching controllers for power transfer regulation of IPT systems has been presented. The self-tuning capability of the proposed controller allows synchronization of the switching operations with the resonance current of the IPT system and enables soft-switching operations to achieve a high efficiency. A simplified design for the proposed controller is introduced which eliminates the need for high-priced DSP/FPGA solutions and enables higher operating frequencies. The simulation results and experimental studies on a case study IPT system show that the proposed controller effectively regulates the resonant current around a desired value, synchronizes the switching operations with the resonant current and enables soft-switching operations. Although the proposed controller is designed for two-stage $\mathrm{AC} / \mathrm{DC} / \mathrm{AC}$ converter topologies, using the same design methodology it can be designed for any type of converter topology.

In Chapter 4, direct single-phase ac-ac matrix converters with self-tuning capability and soft-switching operations for bidirectional inductive power transfer (IPT) systems are introduced. The proposed direct single-phase AC-AC converters along with the proposed power transfer controller can be used as an alternative to conventional two-stage converters for bidirectional IPT systems. Due to the elimination of AC-DC conversion stage and bulky DC-link capacitors, the converter is more compact and reliable compared to conventional two-stage converters. The power transfer controller which is designed based on the energy injection/regeneration 
principle and is implemented based on simplified logic design, effectively enables bidirectional power transfer at 10 different power levels. The resonance frequency tracking capability ensures that the converter operates exactly at the damped resonance frequency of the IPT system and therefore, power transfer efficiency with high efficiency can be achieved. Also, the converter benefits from soft-switching operations that further enhances the efficiency of the converter. Finally, the structure of the single-phase matrix converter along with the simplified controller makes it suitable for residential inductive charging systems with bidirectional power flow capability. The resonance frequency tracking capability of the converter makes it suitable for inductive EV charging systems, in which the system may have variations. The bidirectional power transfer capability can be used to make single-phase G2V and V2G connections through IPT systems at multiple power levels including those defined by SAE J2954 standard.

In Chapter 5, direct three-phase single-stage ac-ac converters for inductive power transfer (IPT) systems are introduced. The proposed converters have different control modes, which can be used to regulate the resonant current, resonant voltage and output power around user-defined reference values. The energy injection and free oscillation control strategy provides a simple and effective method, which makes it suitable for the control of IPT systems. Key benefits of the proposed converter are the minimized number of switches, soft-switching operation, and elimination of short-life electrolytic capacitors. These features will lead to high-efficiency converters with high reliability and extended lifetime. Specifically, with the use of reverse blocking switches, a direct AC-AC matrix converter is developed and built only with seven switches. The simulation results and experimental studies show that the proposed converters can maintain the resonant current or the output power around the user-defined reference values. This feature makes the converters suitable for dy- 
namic IPT systems, where the system has inherent variations. The simulation and experimental results on the proposed converters with power controller show that the converters can effectively establish a bidirectional power transfer connection between the IPT system and the power grid at a desired power level making it suitable for forming contactless G2V and V2G connections. The converter achieves zero-current switching (soft-switching) and can track the resonance frequency in dynamically varying IPT systems which both together ensure the high efficiency operation of the system. The soft-switching operations significantly reduce the complexity of the controller design by eliminating the need for multi-stage commutation processes for bidirectional switches. Moreover, the controller is designed based on a simplified switching logic which can be easily implemented on FPGA boards without the need for DSP platforms.

In Chapter 6, further applications of the self-tuning controllers for inductive EV charging systems are introduced. These applications include conductive foreign object detection (FOD) and vehicle detection in inductive EV charging systems. The proposed FOD method based on the resonance frequency deviation provides a fast and effective technique for detection of conductive foreign objects in IPT systems. By the use of self-tuning controllers, the need for detection sensors is eliminated, which as a result simplifies the implementation of the method. The proposed technique can be implemented for initial (standby) detection at low-power and online detection at full-power. Experimental results on a case study IPT system show that the proposed method is effective in detecting conductive objects and in initial detection mode, it can detect a 5-cent coin in the power transfer area in about $200 \mathrm{~ms}$. However, comparisons between the effect of vehicle variations and conductive foreign objects on the resonance frequency deviation show that online detection mode would not be effective for detection of such small objects. The 
detection of larger objects is achieved in both initial detection and online detection modes with much higher speed and larger detection area. Furthermore, it is shown that by reducing the detection speed, the detection area is significantly increased and much smaller conductive objects can be detected. The resonance frequency deviations are proportional to the size and shape of the objects and in fact can be used as a measure for the size of the object.

The proposed vehicle detection method provides a simple and effective mechanism which can be used in inductive charging stations to enable automated inductive charging process. Since the transmitter magnetic structure is used as the detection coil, the proposed method can be implemented in any inductive charging system and requires minor system modifications. The resonance frequency of the inductive charging system is used as a measure for vehicle detection. The resonance frequency tracking capability is enabled using self-tuning controllers for IPT systems which have relatively a simple design. The simulations results based on 2D and $3 \mathrm{D}$ FEA showed that the vehicle can be detected in about 1.5 meters from the perfect alignment and once the resonance frequency recovers to its original value indicates that the vehicle is perfectly aligned and therefore the charging process can be initiated.

\subsection{Future Works}

The following topics are suggested for future works:

\section{Enhancing the Speed of Physics-Based Multi-Objective Optimiza- tion Using Parallel Processing}

The developed physics-based multi-objective optimization of magnetic structures is highly effective for obtaining optimal structures. The only drawback of 
this method is the optimization time. However, using parallel processing the optimization can be performed in a much shorter time. This can be achieved by performing the $2 \mathrm{D}$ or $3 \mathrm{D}$ FEAs for individuals at the same time.

\section{Development of a Universal Power Controller for Resonant Power Converters}

In this dissertation, different power controller methods for various types of power converters have been introduced. Each controller is converter specific and is not compatible with other types of converters. It is possible, however, to design a universal controller that is compatible with different types of power converters. The controller can be designed to cover wide range of converters including AC-DC-AC full-bridge, half-bridge, single-phase and three-phase direct and indirect $\mathrm{AC}-\mathrm{AC}$ matrix converters.

\section{Development of a Universal Power Converter for Inductive Power}

\section{Transfer Systems}

The development of a universal power converter for inductive power transfer systems is proposed. The universal converter should be able to directly convert DC, single-phase and three-phase AC input into high-frequency AC. The input can have a wide-range range of frequency. The converter can benefit from resonance nature of IPT systems to achieve soft-switching operations and

bidirectional power transfer. A converter topology comprised of 6 bidirectional switches forming three input arms similar to Fig. 5.12 is suggested.

\section{Development of Direct Current-fed AC-AC Converters Topologies With Built-in Power Factor Correction}

The AC-AC converter which are developed in this thesis require the use of power filters to current and voltage harmonic mitigation and power factor 
improvement. The development of direct AC-AC Converters topologies with built-in power factor correction are suggested as a future work. This can be achieved developing current-fed matrix converter topologies. The current-fed matrix converters can be developed by adding input inductors to the topology and modifying the developed switching mechanism to cope with new inductive elements. These types of converters are expected inherently have low current harmonics to be more compact.

\section{Applications of Wide-Bandgap Power Electronics Devices for Effi- ciency Improvement of Power Converters in IPT Systems}

Recent advances in wide-bandgap power electronics devices has led to more efficient high-frequency power electronics converters with improved power density. This emerging technology can be applied to IPT systems to further reduce the size of power electronics converters and improve efficiency.

\section{Living Object Detection Methods in IPT Systems by Detection of the Capacitance Between the Transmitter Structure and Ground}

This method is based on the estimation of equivalent capacitance between transmitter and receiver magnetic structures. This method can be used to detect living and conductive objects in the charging area. Due to high amounts of water inside the living bodies, the presence of such objects in the power transfer area will affect the electric field distribution. As a result the equivalent capacitance between primary and ground will change.

In the proposed method the capacitance between the primary and ground is considered as a measure for detection of foreign objects in IPT systems. The equivalent capacitor is used as a part of a circuit to generate a high frequency pulse wave. The frequency of the pulse wave corresponds to the equivalent 
capacitance of the primary pad respect to ground. Therefore, the presence of a foreign object which causes a change in the equivalent capacitance will change the frequency of the pulse wave signal. The presence of a foreign object can be identified by detecting changes in the frequency of the pulse wave signal with respect to its nominal value.

\section{Online Parameter Estimation Techniques Along With Foreign Ob- ject Detection}

One major challenge in IPT systems is to maintain the maximum power transfer efficiency in any operating conditions. The maximum power transfer operating point of an inductive charging system is determined by the primary power electronics controller based on several key parameters including magnetic coupling between transmitter and receiver, airgap and traction battery voltage, and charging requirements. Online parameter estimation in IPT systems can be employed to continuously adapt the IPT system to the target system variations and track the maximum power transfer efficiency. Therefore, online parameter estimation is essential and can significantly contribute to the performance enhancement of IPT systems. Also, parameter estimation methods that do not require information from the receiver side are of more interest as the need for communication between the transmitter and receiver is eliminated.

\section{Use of Machine Learning Algorithms for Vehicle Proximity Detec- tion and Foreign Object Detection}

Intrusion of foreign objects into charging area or the proximity of vehicle each will affect the in a specific pattern. For example, the resonance frequency pattern when vehicle is approaching the charging area is shown in Fig. 6.16. 
Machine learning algorithms can be trained with such patterns for different inductive charging configurations and can be utilized to perform the detection by recognizing the patterns of foreign objects intrusion or vehicle proximity. 


\section{BIBLIOGRAPHY}

[1] P. Sergeant and A. V. D. Bossche, "Inductive coupler for contactless power transmission," IET Electric Power Applications, vol. 2, pp. 1-7, Jan 2008.

[2] J. de Boeij, E. A. Lomonova, and A. J. A. Vandenput, "Optimization of contactless planar actuator with manipulator," IEEE Trans. Magn., vol. 44, pp. 1118-1121, June 2008.

[3] P. Li and R. Bashirullah, "A wireless power interface for rechargeable battery operated medical implants," IEEE Trans. Circuits Syst. II, Exp. Briefs, vol. 54, pp. 912-916, Oct 2007.

[4] G. Wang, W. Liu, M. Sivaprakasam, and G. A. Kendir, "Design and analysis of an adaptive transcutaneous power telemetry for biomedical implants," IEEE Trans. Circuits Syst. I, Reg. Papers, vol. 52, pp. 2109-2117, Oct 2005.

[5] G. A. Covic and J. T. Boys, "Modern trends in inductive power transfer for transportation applications," IEEE J. Emerg. Sel. Top. Power Electron., vol. 1, pp. 28-41, March 2013.

[6] M. Moghaddami, A. Anzalchi, and A. I. Sarwat, "Finite element based design optimization of magnetic structures for roadway inductive power transfer systems," in 2016 IEEE Transportation Electrification Conference and Expo (ITEC), pp. 1-6, June 2016.

[7] C.-S. Wang, O. H. Stielau, and G. A. Covic, "Design considerations for a contactless electric vehicle battery charger," IEEE Trans. Ind. Electron., vol. 52, pp. 1308-1314, Oct 2005.

[8] J. M. Miller and A. Daga, "Elements of wireless power transfer essential to high power charging of heavy duty vehicles," IEEE Trans. Transport. Electrific., vol. 1, pp. 26-39, June 2015.

[9] M. Budhia, G. Covic, and J. Boys, "Design and optimization of circular magnetic structures for lumped inductive power transfer systems," Power Electronics, IEEE Transactions on, vol. 26, pp. 3096-3108, Nov 2011.

[10] G. Buja, C. T. Rim, and C. C. Mi, "Dynamic charging of electric vehicles by wireless power transfer," IEEE Trans. Ind. Electron., vol. 63, pp. 6530-6532, Oct 2016. 
[11] G. Buja, M. Bertoluzzo, and H. K. Dashora, "Lumped track layout design for dynamic wireless charging of electric vehicles," IEEE Trans. Ind. Electron., vol. 63, pp. 6631-6640, Oct 2016.

[12] C. C. Mi, G. Buja, S. Y. Choi, and C. T. Rim, "Modern advances in wireless power transfer systems for roadway powered electric vehicles," IEEE Trans. Ind. Electron., vol. 63, pp. 6533-6545, Oct 2016.

[13] T. V. Theodoropoulos, I. G. Damousis, and A. J. Amditis, "Demand-side management ict for dynamic wireless ev charging," IEEE Trans. Ind. Electron., vol. 63, pp. 6623-6630, Oct 2016.

[14] J. H. Kim, B. S. Lee, J. H. Lee, S. H. Lee, C. B. Park, S. M. Jung, S. G. Lee, K. P. Yi, and J. Baek, "Development of 1-mw inductive power transfer system for a high-speed train," IEEE Trans. Ind. Electron., vol. 62, pp. 6242-6250, Oct 2015.

[15] C. T. Rim and C. Mi, Introduction to Static Charging. IEEE, 2017.

[16] S. Lukic and Z. Pantic, "Cutting the cord: Static and dynamic inductive wireless charging of electric vehicles," IEEE Electrific. Mag., vol. 1, pp. 5764, Sept 2013.

[17] J. M. Miller, O. C. Onar, and M. Chinthavali, "Primary-side power flow control of wireless power transfer for electric vehicle charging," IEEE J. Emerg. Sel. Top. Power Electron., vol. 3, pp. 147-162, March 2015.

[18] S. Moon and G. Moon, "Wireless power transfer system with an asymmetric four-coil resonator for electric vehicle battery chargers," IEEE Transactions on Power Electronics, vol. 31, pp. 6844-6854, Oct 2016.

[19] A. Q. Cai and L. Siek, "A 2-kw, 95using gallium nitride gate injection transistors," IEEE Journal of Emerging and Selected Topics in Power Electronics, vol. 5, pp. 458-468, March 2017.

[20] Q. Zhu, Y. Zhang, C. Liao, Y. Guo, L. Wang, and F. Li, "Experimental study on asymmetric wireless power transfer system for electric vehicle considering ferrous chassis," IEEE Transactions on Transportation Electrification, vol. 3, pp. 427-433, June 2017. 
[21] T. Diekhans and R. W. D. Doncker, "A dual-side controlled inductive power transfer system optimized for large coupling factor variations and partial load," IEEE Transactions on Power Electronics, vol. 30, pp. 6320-6328, Nov 2015.

[22] J. Deng, W. Li, T. D. Nguyen, S. Li, and C. C. Mi, "Compact and efficient bipolar coupler for wireless power chargers: Design and analysis," IEEE Transactions on Power Electronics, vol. 30, pp. 6130-6140, Nov 2015.

[23] F. Y. Lin, G. A. Covic, and J. T. Boys, "Evaluation of magnetic pad sizes and topologies for electric vehicle charging," IEEE Transactions on Power Electronics, vol. 30, pp. 6391-6407, Nov 2015.

[24] K. A. Kalwar, M. Aamir, and S. Mekhilef, "Inductively coupled power transfer (icpt) for electric vehicle charging - a review," Renewable and Sustainable Energy Reviews, vol. 47, pp. $462-475,2015$.

[25] P. K. Joseph and D. Elangovan, "A review on renewable energy powered wireless power transmission techniques for light electric vehicle charging applications," Journal of Energy Storage, vol. 16, pp. 145 - 155, 2018.

[26] J. L. Villa, J. Sallán, A. Llombart, and J. F. Sanz, "Design of a high frequency inductively coupled power transfer system for electric vehicle battery charge," Applied Energy, vol. 86, no. 3, pp. 355 - 363, 2009.

[27] D. P. Kar, S. S. Biswal, P. K. Sahoo, P. P. Nayak, and S. Bhuyan, "Selection of maximum power transfer region for resonant inductively coupled wireless charging system," AEU - International Journal of Electronics and Communications, vol. 84, pp. $84-92,2018$.

[28] D. Iannuzzi, L. Rubino, L. P. D. Noia, G. Rubino, and P. Marino, "Resonant inductive power transfer for an e-bike charging station," Electric Power Systems Research, vol. 140, pp. 631 - 642, 2016.

[29] Y. J. Jang, "Survey of the operation and system study on wireless charging electric vehicle systems," Transportation Research Part C: Emerging Technologies, 2018.

[30] C. T. Rim, "34 - wireless charging of electric vehicles," in Power Electronics Handbook (Fourth Edition) (M. H. Rashid, ed.), pp. 1113 - 1137, ButterworthHeinemann, fourth edition ed., 2018. 
[31] "Design optimisation for an efficient wireless power transfer system for electric vehicles," Energy Procedia, vol. 117, pp. 1015 - 1023, 2017.

[32] M. Fuller, "Wireless charging in california: Range, recharge, and vehicle electrification," Transportation Research Part C: Emerging Technologies, vol. 67, pp. $343-356,2016$.

[33] K. A. Kalwar, M. Aamir, and S. Mekhilef, "A design method for developing a high misalignment tolerant wireless charging system for electric vehicles," Measurement, vol. 118, pp. 237 - 245, 2018.

[34] R. Riemann, D. Z. Wang, and F. Busch, "Optimal location of wireless charging facilities for electric vehicles: Flow-capturing location model with stochastic user equilibrium," Transportation Research Part C: Emerging Technologies, vol. 58, pp. 1 - 12, 2015.

[35] L. Sun, D. Ma, and H. Tang, "A review of recent trends in wireless power transfer technology and its applications in electric vehicle wireless charging," Renewable and Sustainable Energy Reviews, vol. 91, pp. 490 - 503, 2018.

[36] C. Panchal, S. Stegen, and J. Lu, "Review of static and dynamic wireless electric vehicle charging system," Engineering Science and Technology, an International Journal, 2018.

[37] Z. Chen, W. Liu, and Y. Yin, "Deployment of stationary and dynamic charging infrastructure for electric vehicles along traffic corridors," Transportation Research Part C: Emerging Technologies, vol. 77, pp. 185-206, 2017.

[38] S. A. Birrell, D. Wilson, C. P. Yang, G. Dhadyalla, and P. Jennings, "How driver behaviour and parking alignment affects inductive charging systems for electric vehicles," Transportation Research Part C: Emerging Technologies, vol. 58, pp. $721-731,2015$. Technologies to support green driving.

[39] I. Karakitsios, E. Karfopoulos, and N. Hatziargyriou, "Impact of dynamic and static fast inductive charging of electric vehicles on the distribution network," Electric Power Systems Research, vol. 140, pp. 107 - 115, 2016.

[40] W. Zhang, S. C. Wong, C. K. Tse, and Q. Chen, "An optimized track length in roadway inductive power transfer systems," IEEE Journal of Emerging and Selected Topics in Power Electronics, vol. 2, pp. 598-608, Sept 2014. 
[41] S. Jeong, Y. J. Jang, and D. Kum, "Economic analysis of the dynamic charging electric vehicle," IEEE Transactions on Power Electronics, vol. 30, pp. 63686377, Nov 2015.

[42] X. Zhang, Z. Yuan, Q. Yang, Y. Li, J. Zhu, and Y. Li, "Coil design and efficiency analysis for dynamic wireless charging system for electric vehicles," IEEE Transactions on Magnetics, vol. 52, pp. 1-4, July 2016.

[43] S. Y. Jeong, J. H. Park, G. P. Hong, and C. T. Rim, "Auto-tuning control by variation of self-inductance with small air gap for dynamic wireless ev charging," IEEE Transactions on Power Electronics, pp. 1-1, 2018.

[44] A. Ahmad, M. S. Alam, and R. Chabaan, "A comprehensive review of wireless charging technologies for electric vehicles," IEEE Transactions on Transportation Electrification, vol. 4, pp. 38-63, March 2018.

[45] Z. Zhang, H. Pang, C. H. T. Lee, X. Xu, X. Wei, and J. Wang, "Comparative analysis and optimization of dynamic charging coils for roadway-powered electric vehicles," IEEE Transactions on Magnetics, vol. 53, pp. 1-6, Nov 2017.

[46] X. Mou and H. Sun, "Analysis of multiple segmented transmitters design in dynamic wireless power transfer for electric vehicles charging," Electronics Letters, vol. 53, no. 14, pp. 941-943, 2017.

[47] M. Pazos-Revilla, A. Alsharif, S. Gunukula, T. N. Guo, M. Mahmoud, and $\mathrm{X}$. Shen, "Secure and privacy-preserving physical-layer-assisted scheme for ev dynamic charging system," IEEE Transactions on Vehicular Technology, vol. 67, pp. 3304-3318, April 2018.

[48] H. Liu, X. Huang, L. Tan, J. Guo, W. Wang, C. Yan, and C. Xu, "Dynamic wireless charging for inspection robots based on decentralized energy pickup structure," IEEE Transactions on Industrial Informatics, vol. 14, pp. 17861797, April 2018.

[49] Q. Zhu, L. Wang, Y. Guo, C. Liao, and F. Li, "Applyinglcccompensation network to dynamic wireless ev charging system," IEEE Transactions on Industrial Electronics, vol. 63, pp. 6557-6567, Oct 2016.

[50] R. Tavakoli and Z. Pantic, "Analysis, design, and demonstration of a 25$\mathrm{kw}$ dynamic wireless charging system for roadway electric vehicles," IEEE $J$. Emerg. Sel. Top. Power Electron., vol. 6, pp. 1378-1393, Sept 2018. 
[51] Y. Liu, R. Mai, D. Liu, Y. Li, and Z. He, "Efficiency optimization for wireless dynamic charging system with overlapped dd coil arrays," IEEE Transactions on Power Electronics, vol. 33, pp. 2832-2846, April 2018.

[52] X. Dai, J. Jiang, and J. Wu, "Charging area determining and power enhancement method for multi-excitation unit configuration of wirelessly dynamic charging ev system," IEEE Transactions on Industrial Electronics, pp. 1-1, 2018.

[53] Y. Guo, L. Wang, Q. Zhu, C. Liao, and F. Li, "Switch-on modeling and analysis of dynamic wireless charging system used for electric vehicles," IEEE Transactions on Industrial Electronics, vol. 63, pp. 6568-6579, Oct 2016.

[54] I. Hwang, Y. J. Jang, Y. D. Ko, and M. S. Lee, "System optimization for dynamic wireless charging electric vehicles operating in a multiple-route environment," IEEE Transactions on Intelligent Transportation Systems, vol. 19, pp. 1709-1726, June 2018.

[55] X. Liu, W. Han, C. Liu, and P. W. T. Pong, "Marker-free coil-misalignment detection approach using tmr sensor array for dynamic wireless charging of electric vehicles," IEEE Transactions on Magnetics, pp. 1-5, 2018.

[56] S. Jeong, Y. J. Jang, D. Kum, and M. S. Lee, "Charging automation for electric vehicles: Is a smaller battery good for the wireless charging electric vehicles?," IEEE Transactions on Automation Science and Engineering, pp. 1-12, 2018.

[57] S. Zhou and C. C. Mi, "Multi-paralleled lcc reactive power compensation networks and their tuning method for electric vehicle dynamic wireless charging," IEEE Transactions on Industrial Electronics, vol. 63, pp. 6546-6556, Oct 2016.

[58] J. M. Miller, O. C. Onar, C. White, S. Campbell, C. Coomer, L. Seiber, R. Sepe, and A. Steyerl, "Demonstrating dynamic wireless charging of an electric vehicle: The benefit of electrochemical capacitor smoothing," IEEE Power Electronics Magazine, vol. 1, pp. 12-24, March 2014.

[59] D. Patil, M. K. McDonough, J. M. Miller, B. Fahimi, and P. T. Balsara, "Wireless power transfer for vehicular applications: Overview and challenges," IEEE Transactions on Transportation Electrification, vol. 4, pp. 3-37, March 2018. 
[60] F. Lu, H. Zhang, H. Hofmann, and C. C. Mi, "A dynamic charging system with reduced output power pulsation for electric vehicles," IEEE Trans. Ind. Electron., vol. 63, pp. 6580-6590, Oct 2016.

[61] A. Kamineni, M. J. Neath, A. Zaheer, G. A. Covic, and J. T. Boys, "Interoperable ev detection for dynamic wireless charging with existing hardware and free resonance," IEEE Transactions on Transportation Electrification, vol. 3, pp. 370-379, June 2017.

[62] Z. Wang, S. Cui, S. Han, K. Song, C. Zhu, M. I. Matveevich, and O. S. Yurievich, "A novel magnetic coupling mechanism for dynamic wireless charging system for electric vehicles," IEEE Transactions on Vehicular Technology, vol. 67, pp. 124-133, Jan 2018.

[63] J. M. Miller, P. T. Jones, J. Li, and O. C. Onar, "Ornl experience and challenges facing dynamic wireless power charging of ev's," IEEE Circuits and Systems Magazine, vol. 15, pp. 40-53, Secondquarter 2015.

[64] A. Zaheer, M. Neath, H. Z. Z. Beh, and G. A. Covic, "A dynamic ev charging system for slow moving traffic applications," IEEE Transactions on Transportation Electrification, vol. 3, pp. 354-369, June 2017.

[65] L. Xiang, X. Li, J. Tian, and Y. Tian, "A crossed dd geometry and its doublecoil excitation method for electric vehicle dynamic wireless charging systems," IEEE Access, vol. 6, pp. 45120-45128, 2018.

[66] S. Li, Z. Liu, H. Zhao, L. Zhu, C. Shuai, and Z. Chen, "Wireless power transfer by electric field resonance and its application in dynamic charging," IEEE Transactions on Industrial Electronics, vol. 63, pp. 6602-6612, Oct 2016.

[67] H. Li, G. Dán, and K. Nahrstedt, "Portunes+: Privacy-preserving fast authentication for dynamic electric vehicle charging," IEEE Transactions on Smart Grid, vol. 8, pp. 2305-2313, Sept 2017.

[68] S. Cui, Z. Wang, S. Han, C. Zhu, and C. C. Chan, "Analysis and design of multiphase receiver with reduction of output fluctuation for ev dynamic wireless charging system," IEEE Transactions on Power Electronics, pp. 1-1, 2018.

[69] T. Fujita, T. Yasuda, and H. Akagi, "A dynamic wireless power transfer system applicable to a stationary system," IEEE Transactions on Industry Applications, vol. 53, pp. 3748-3757, July 2017. 
[70] A. Kamineni, M. J. Neath, G. A. Covic, and J. T. Boys, "A mistuning-tolerant and controllable power supply for roadway wireless power systems," IEEE Transactions on Power Electronics, vol. 32, pp. 6689-6699, Sept 2017.

[71] C. Park, S. Lee, S. Y. Jeong, G. Cho, and C. T. Rim, "Uniform power i-type inductive power transfer system withdq-power supply rails for on-line electric vehicles," IEEE Transactions on Power Electronics, vol. 30, pp. 6446-6455, Nov 2015.

[72] S. Y. Choi, B. W. Gu, S. Y. Jeong, and C. T. Rim, "Advances in wireless power transfer systems for roadway-powered electric vehicles," IEEE Journal of Emerging and Selected Topics in Power Electronics, vol. 3, pp. 18-36, March 2015 .

[73] J. Huh, S. W. Lee, W. Y. Lee, G. H. Cho, and C. T. Rim, "Narrow-width inductive power transfer system for online electrical vehicles," IEEE Transactions on Power Electronics, vol. 26, pp. 3666-3679, Dec 2011.

[74] S. Y. Choi, S. Y. Jeong, B. W. Gu, G. C. Lim, and C. T. Rim, "Ultraslim s-type power supply rails for roadway-powered electric vehicles," IEEE Transactions on Power Electronics, vol. 30, pp. 6456-6468, Nov 2015.

[75] I. Karakitsios, F. Palaiogiannis, A. Markou, and N. D. Hatziargyriou, "Optimizing the energy transfer, with a high system efficiency in dynamic inductive charging of evs," IEEE Transactions on Vehicular Technology, vol. 67, pp. 4728-4742, June 2018.

[76] Y. J. Jang, E. S. Suh, and J. W. Kim, "System architecture and mathematical models of electric transit bus system utilizing wireless power transfer technology," IEEE Systems Journal, vol. 10, pp. 495-506, June 2016.

[77] S. Lee, B. Choi, and C. T. Rim, "Dynamics characterization of the inductive power transfer system for online electric vehicles by laplace phasor transform," IEEE Transactions on Power Electronics, vol. 28, pp. 5902-5909, Dec 2013.

[78] S. Jung, H. Lee, C. S. Song, J. Han, W. Han, and G. Jang, "Optimal operation plan of the online electric vehicle system through establishment of a dc distribution system," IEEE Transactions on Power Electronics, vol. 28, pp. 5878-5889, Dec 2013.

[79] F. Chen, N. Taylor, R. Balieu, and N. Kringos, "Dynamic application of the inductive power transfer (ipt) systems in an electrified road: Dielectric 
power loss due to pavement materials," Construction and Building Materials, vol. 147 , pp. $9-16,2017$.

[80] P. Venugopal, A. Shekhar, E. Visser, N. Scheele, G. R. C. Mouli, P. Bauer, and S. Silvester, "Roadway to self-healing highways with integrated wireless electric vehicle charging and sustainable energy harvesting technologies," Applied Energy, vol. 212, pp. 1226 - 1239, 2018.

[81] F. Deflorio, P. Guglielmi, I. Pinna, L. Castello, and S. Marfull, "Modeling and analysis of wireless "charge while driving" operations for fully electric vehicles," Transportation Research Procedia, vol. 5, pp. 161 - 174, 2015. SIDT Scientific Seminar 2013.

[82] Y. D. Ko and Y. J. Jang, "Efficient design of an operation profile for wireless charging electric tram systems," Computers and Industrial Engineering, 2018.

[83] J. He, H. Yang, H.-J. Huang, and T.-Q. Tang, "Impacts of wireless charging lanes on travel time and energy consumption in a two-lane road system," Physica A: Statistical Mechanics and its Applications, vol. 500, pp. 1 - 10, 2018.

[84] R. Bosshard and J. W. Kolar, "Multi-objective optimization of $50 \mathrm{kw} / 85 \mathrm{khz}$ ipt system for public transport," IEEE J. Emerg. Sel. Topics Power Electron., vol. 4, pp. 1370-1382, Dec 2016.

[85] Z. N. Low, R. A. Chinga, R. Tseng, and J. Lin, "Design and test of a highpower high-efficiency loosely coupled planar wireless power transfer system," IEEE Trans. Ind. Electron., vol. 56, pp. 1801-1812, May 2009.

[86] S. Wang and D. G. Dorrell, "Copper loss analysis of ev charging coupler," IEEE Transactions on Magnetics, vol. 51, pp. 1-4, Nov 2015.

[87] S. Wang and D. G. Dorrell, "Loss analysis of circular wireless ev charging coupler," IEEE Transactions on Magnetics, vol. 50, pp. 1-4, Nov 2014.

[88] P. Raval, D. Kacprzak, and A. P. Hu, "Analysis of flux leakage of a 3-d inductive power transfer system," IEEE J. Emerging and Selected Topics in Power Electronics, vol. 3, pp. 205-214, March 2015.

[89] R. Bosshard, J. Kolar, J. Muhlethaler, I. Stevanovic, B. Wunsch, and F. Canales, "Modeling and $\eta-\alpha$-pareto optimization of inductive power 
transfer coils for electric vehicles," IEEE J. Emerg. Sel. Topics Power Electron., vol. 3, pp. 50-64, March 2015.

[90] SAE International, "J2954," Wireless Power Transfer for Light-Duty PlugIn/Electric Vehicles and Alignment Methodology, 2016.

[91] J. Lin, R. Saunders, K. Schulmeister, P. Söderberg, A. Swerdlow, M. Taki, B. Veyret, G. Ziegelberger, M. H. Repacholi, R. Matthes, et al., "Icnirp guidelines for limiting exposure to time-varying electric and magnetic fields (1 hz to 100 khz).," Health Physics, vol. 99, pp. 818-836, 2010.

[92] J. P. C. Smeets, T. T. Overboom, J. W. Jansen, and E. A. Lomonova, "Inductance calculation nearby conducting material," IEEE Trans. Magn., vol. 50, pp. 1-4, Nov 2014.

[93] S. Y. Hui, "Planar wireless charging technology for portable electronic products and qi," Proceedings of the IEEE, vol. 101, pp. 1290-1301, June 2013.

[94] F. Turki, A. Körner, J. Tlatlik, and A. Brown, "Compact, safe and efficient wireless and inductive charging for plug-in hybrids and electric vehicles," $S A E$ International Journal of Alternative Powertrains, vol. 3, no. 2014-01-1892, pp. 139-151, 2014.

[95] Y. Bu, M. Nishiyama, T. Ueda, Y. Tashima, and T. Mizuno, "Examination of wireless power transfer combined with the utilization of distance detection," IEEE Trans. Magn., vol. 50, pp. 1-4, Nov 2014.

[96] K. Hall, M. Kesler, R. Fiorello, D. Schatz, K. Kulikowski, and M. Soljacic, "Vehicle charger safety system and method," Mar. 31 2011. US Patent App. $12 / 899,281$.

[97] P. Hoffman, R. Boyer, and R. Henderson, "Foreign object detection system and method suitable for source resonator of wireless energy transfer system," Apr. 5 2016. US Patent 9,304,042.

[98] A. Roy, N. Katz, A. Kurs, C. Buenrostro, S. Verghese, M. Kesler, K. Hall, and H. Lou, "Foreign object detection in wireless energy transfer systems," Aug. 2 2016. US Patent 9,404,954.

[99] A. Karanth, K. Hara, and R. Kumar, "Foreign object detection in inductive coupled wireless power transfer environment using thermal sensors," Jan. 12 2012. WO Patent App. PCT/EP2011/059,839. 
[100] S. Verghese, M. Kesler, K. Hall, and H. Lou, "Foreign object detection in wireless energy transfer systems," Mar. 21 2013. US Patent App. 13/608,956.

[101] M. R. Sonapreetha, S. Y. Jeong, S. Y. Choi, and C. T. Rim, "Dual-purpose non-overlapped coil sets as foreign object and vehicle location detections for wireless stationary ev chargers," in 2015 IEEE PELS Workshop on Emerging Technologies: Wireless Power (2015 WoW), pp. 1-7, June 2015.

[102] W. Von Novak, P. Monat, and E. Kallal, "Wireless power system with capacitive proximity sensing," Oct. 6 2015. US Patent 9,154,189.

[103] N. Kuyvenhoven, C. Dean, J. Melton, J. Schwannecke, and A. E. Umenei, "Development of a foreign object detection and analysis method for wireless power systems," in Product Compliance Engineering (PSES), 2011 IEEE Symposium on, pp. 1-6, Oct 2011.

[104] V. Muratov and E. Oettinger, "Systems and methods of wireless power transfer with interference detection," Mar. 22 2016. US Patent 9,294,153.

[105] X. Liu, L. Swaans, K. Chan, H. Low, and W. Chan, "Methods and systems for detecting foreign objects in a wireless charging system," Nov. 3 2015. US Patent 9,178,361.

[106] D. A. G. Pedder, A. D. Brown, and J. A. Skinner, "A contactless electrical energy transmission system," IEEE Trans. Ind. Electron., vol. 46, pp. 23-30, Feb 1999.

[107] C.-G. Kim, D.-H. Seo, J.-S. You, J.-H. Park, and B. H. Cho, "Design of a contactless battery charger for cellular phone," IEEE Trans. Ind. Electron., vol. 48, pp. 1238-1247, Dec 2001.

[108] G. A. J. Elliott, G. A. Covic, D. Kacprzak, and J. T. Boys, "A new concept: Asymmetrical pick-ups for inductively coupled power transfer monorail systems," IEEE Transactions on Magnetics, vol. 42, pp. 3389-3391, Oct 2006.

[109] H. Sakamoto, K. Harada, S. Washimiya, K. Takehara, Y. Matsuo, and F. Nakao, "Large air-gap coupler for inductive charger [for electric vehicles]," IEEE Transactions on Magnetics, vol. 35, pp. 3526-3528, Sep 1999.

[110] S. Valtchev, B. Borges, K. Brandisky, and J. B. Klaassens, "Resonant contactless energy transfer with improved efficiency," IEEE Trans. Power Electron., vol. 24, pp. 685-699, March 2009. 
[111] X. Liu and S. Y. Hui, "Optimal design of a hybrid winding structure for planar contactless battery charging platform," IEEE Trans. Power Electron., vol. 23, pp. 455-463, Jan 2008.

[112] Z. Luo and X. Wei, "Analysis of square and circular planar spiral coils in wireless power transfer system for electric vehicles," IEEE Trans. Ind. Electron., vol. 65, pp. 331-341, Jan 2018.

[113] J. Acero, C. Carretero, I. Lope, R. Alonso, . Lucia, and J. M. Burdio, "Analysis of the mutual inductance of planar-lumped inductive power transfer systems," IEEE Trans. Ind. Electron., vol. 60, pp. 410-420, Jan 2013.

[114] A. Zaheer, G. A. Covic, and D. Kacprzak, "A bipolar pad in a 10-khz 300w distributed ipt system for agv applications," IEEE Trans. Ind. Electron., vol. 61, pp. 3288-3301, July 2014.

[115] S. Kim, G. A. Covic, and J. T. Boys, "Tripolar pad for inductive power transfer systems for ev charging," IEEE Trans. Power Electron., vol. 32, pp. 5045-5057, July 2017.

[116] R. Lai, F. Wang, R. Burgos, Y. Pei, D. Boroyevich, B. Wang, T. A. Lipo, V. D. Immanuel, and K. J. Karimi, "A systematic topology evaluation methodology for high-density three-phase pwm ac-ac converters," IEEE Trans. Power Electron., vol. 23, pp. 2665-2680, Nov 2008.

[117] B.-G. Gu and K. Nam, "A dc-link capacitor minimization method through direct capacitor current control," IEEE Trans. Ind. Appl., vol. 42, pp. 573581, March 2006.

[118] L. Li and D. Tang, "Cascade three-level ac/ac direct converter," IEEE Trans. Ind. Electron., vol. 59, pp. 27-34, Jan 2012.

[119] L. Malesani, L. Rossetto, P. Tenti, and P. Tomasin, "Ac/dc/ac pwm converter with reduced energy storage in the dc link," IEEE Trans. Ind. Appl., vol. 31, pp. 287-292, Mar 1995.

[120] P. W. Wheeler, J. Rodriguez, J. C. Clare, L. Empringham, and A. Weinstein, "Matrix converters: a technology review," IEEE Trans. Ind. Electron., vol. 49, pp. 276-288, Apr 2002. 
[121] J. W. Kolar, T. Friedli, J. Rodriguez, and P. W. Wheeler, "Review of threephase pwm ac-ac converter topologies," IEEE Trans. Ind. Electron., vol. 58, pp. 4988-5006, Nov 2011.

[122] J. Rodriguez, M. Rivera, J. W. Kolar, and P. W. Wheeler, "A review of control and modulation methods for matrix converters," IEEE Trans. Ind. Electron., vol. 59, pp. 58-70, Jan 2012.

[123] H. Keyhani and H. A. Toliyat, "A soft-switched three-phase ac-ac converter with a high-frequency ac link," IEEE Trans. Ind. Appl., vol. 50, pp. 2637-2647, July 2014.

[124] H. L. Li, A. P. Hu, and G. A. Covic, "A direct ac-ac converter for inductive power-transfer systems," IEEE Trans. Power Electron., vol. 27, pp. 661-668, Feb 2012.

[125] U. K. Madawala and D. J. Thrimawithana, "A bidirectional inductive power interface for electric vehicles in v2g systems," IEEE Trans. Ind. Electron., vol. 58, pp. 4789-4796, Oct 2011.

[126] C.-S. Wang, G. A. Covic, and O. H. Stielau, "Investigating an lcl load resonant inverter for inductive power transfer applications," IEEE Trans. Power Electron., vol. 19, pp. 995-1002, July 2004.

[127] C. Y. Huang, J. T. Boys, and G. A. Covic, "Lcl pickup circulating current controller for inductive power transfer systems," IEEE Trans. Power Electron., vol. 28, pp. 2081-2093, April 2013.

[128] S. Aldhaher, P. C. K. Luk, A. Bati, and J. F. Whidborne, "Wireless power transfer using class e inverter with saturable dc-feed inductor," IEEE Trans. Ind. Appl., vol. 50, pp. 2710-2718, July 2014.

[129] Z. Pantic, S. Bai, and S. M. Lukic, "Zcs lcc-compensated resonant inverter for inductive-power-transfer application," IEEE Trans. Ind. Electron., vol. 58, pp. 3500-3510, Aug 2011.

[130] J. I. Rodriguez and S. B. Leeb, "A multilevel inverter topology for inductively coupled power transfer," IEEE Trans. Power Electron., vol. 21, pp. 1607-1617, Nov 2006. 
[131] N. A. Keeling, G. A. Covic, and J. T. Boys, "A unity-power-factor ipt pickup for high-power applications," IEEE Trans. Ind. Electron., vol. 57, pp. 744-751, Feb 2010.

[132] M. G. Egan, D. L. O'Sullivan, J. G. Hayes, M. J. Willers, and C. P. Henze, "Power-factor-corrected single-stage inductive charger for electric vehicle batteries," IEEE Trans. Ind. Electron., vol. 54, pp. 1217-1226, April 2007.

[133] M. Moghaddami, A. Anzalchi, and A. I. Sarwat, "Single-stage three-phase acac matrix converter for inductive power transfer systems," IEEE Trans. Ind. Electron., vol. 63, pp. 6613-6622, Oct 2016.

[134] A. Namadmalan, "Bidirectional current-fed resonant inverter for contactless energy transfer systems," IEEE Trans. Ind. Electron., vol. 62, pp. 238-245, Jan 2015.

[135] U. K. Madawala, M. Neath, and D. J. Thrimawithana, "A power-frequency controller for bidirectional inductive power transfer systems," IEEE Trans. Ind. Electron., vol. 60, pp. 310-317, Jan 2013.

[136] M. J. Neath, A. K. Swain, U. K. Madawala, and D. J. Thrimawithana, "An optimal pid controller for a bidirectional inductive power transfer system using multiobjective genetic algorithm," IEEE Trans. Power Electron., vol. 29, pp. 1523-1531, March 2014.

[137] J. Y. Lee and B. M. Han, "A bidirectional wireless power transfer ev charger using self-resonant pwm," IEEE Trans. Power Electron., vol. 30, pp. 17841787, April 2015.

[138] B. X. Nguyen, D. M. Vilathgamuwa, G. H. B. Foo, P. Wang, A. Ong, U. K. Madawala, and T. D. Nguyen, "An efficiency optimization scheme for bidirectional inductive power transfer systems," IEEE Trans. Power Electron., vol. 30, pp. 6310-6319, Nov 2015.

[139] S. Weerasinghe, U. K. Madawala, and D. J. Thrimawithana, "A matrix converter-based bidirectional contactless grid interface," IEEE Trans. Power Electron., vol. 32, pp. 1755-1766, March 2017.

[140] M. Ishigaki, J. Shin, and E. M. Dede, "A novel soft switching bidirectional dc-dc converter using magnetic and capacitive hybrid power transfer," IEEE Transactions on Power Electronics, vol. 32, pp. 6961-6970, Sept 2017. 
[141] S. Samanta, A. K. Rathore, and D. J. Thrimawithana, "Bidirectional currentfed half-bridge (c) (lc)-(lc ) configuration for inductive wireless power transfer system," IEEE Transactions on Industry Applications, vol. 53, pp. 4053-4062, July 2017.

[142] Y. Tang, Y. Chen, U. K. Madawala, D. J. Thrimawithana, and H. Ma, "A new controller for bidirectional wireless power transfer systems," IEEE Transactions on Power Electronics, vol. 33, pp. 9076-9087, Oct 2018.

[143] S. G. Cimen, A. Pfannkuchen, and B. Schmuelling, "Compensation considerations for bidirectional inductive charging systems of electric vehicles with coil positioning flexibility," IEEE Transactions on Magnetics, vol. 52, pp. 1-4, March 2016.

[144] A. K. Swain, S. Devarakonda, and U. K. Madawala, "Modeling, sensitivity analysis, and controller synthesis of multipickup bidirectional inductive power transfer systems," IEEE Transactions on Industrial Informatics, vol. 10, pp. 1372-1380, May 2014.

[145] A. A. S. Mohamed, A. A. Marim, and O. A. Mohammed, "Magnetic design considerations of bidirectional inductive wireless power transfer system for ev applications," IEEE Transactions on Magnetics, vol. 53, pp. 1-5, June 2017.

[146] N. S. González-Santini, H. Zeng, Y. Yu, and F. Z. Peng, "Z-source resonant converter with power factor correction for wireless power transfer applications," IEEE Trans. Power Electron., vol. 31, pp. 7691-7700, Nov 2016.

[147] S. Weerasinghe, U. K. Madawala, and D. J. Thrimawithana, "A matrix converter-based bidirectional contactless grid interface," IEEE Trans. Power Electron., vol. 32, pp. 1755-1766, March 2017.

[148] L. G. G. de Vicuna, M. Castilla, J. Miret, J. Matas, and J. M. Guerrero, "Sliding-mode control for a single-phase ac/ac quantum resonant converter," IEEE Trans. Ind. Electron., vol. 56, pp. 3496-3504, Sept 2009.

[149] C. T. Rim and G. H. Cho, "Quantum transformation: the analysis of quantum rectifier-inverters," in Conference Record of the IEEE Industry Applications Society Annual Meeting,, pp. 1081-1085 vol.1, Oct 1989.

[150] Z. U. Zahid, Z. M. Dalala, C. Zheng, R. Chen, W. E. Faraci, J. S. J. Lai, G. Lisi, and D. Anderson, "Modeling and control of series-series compensated 
inductive power transfer system," IEEE J. Emerg. Sel. Top. Power Electron., vol. 3, pp. 111-123, March 2015.

[151] A. Berger, M. Agostinelli, S. Vesti, J. A. Oliver, J. A. Cobos, and M. Huemer, "A wireless charging system applying phase-shift and amplitude control to maximize efficiency and extractable power," IEEE Trans. Power Electron., vol. 30, pp. 6338-6348, Nov 2015.

[152] J. T. Matysik, "The current and voltage phase shift regulation in resonant converters with integration control," IEEE Trans. Ind. Electron., vol. 54, pp. 1240-1242, April 2007.

[153] Z. H. Wang, Y. P. Li, Y. Sun, C. S. Tang, and X. Lv, "Load detection model of voltage-fed inductive power transfer system," IEEE Trans. Power Electron., vol. 28, pp. 5233-5243, Nov 2013.

[154] F. F. A. van der Pijl, M. Castilla, and P. Bauer, "Adaptive sliding-mode control for a multiple-user inductive power transfer system without need for communication," IEEE Trans. Ind. Electron., vol. 60, pp. 271-279, Jan 2013.

[155] M. Castilla, L. G. de Vicuna, J. M. Guerrero, J. Matas, and J. Miret, "Slidingmode control of quantum series-parallel resonant converters via input-output linearization," IEEE Trans. Ind. Electron., vol. 52, pp. 566-575, April 2005.

[156] X. Chen, T. Fukuda, and K. D. Young, "Adaptive quasi-sliding-mode tracking control for discrete uncertain input-output systems," IEEE Trans. Ind. Electron., vol. 48, pp. 216-224, Feb 2001.

[157] A. Namadmalan, "Self-oscillating tuning loops for series resonant inductive power transfer systems," IEEE Trans. Power Electron., vol. 31, pp. 73207327, Oct 2016.

[158] A. Namadmalan and J. S. Moghani, "Tunable self-oscillating switching technique for current source induction heating systems," IEEE Trans. Ind. Electron., vol. 61, pp. 2556-2563, May 2014.

[159] Z. Zhang, K. T. Chau, C. Qiu, and C. Liu, "Energy encryption for wireless power transfer," IEEE Trans. Power Electron., vol. 30, pp. 5237-5246, Sept 2015. 
[160] H. L. Li, A. P. Hu, and G. A. Covic, "Primary current generation for a contactless power transfer system using free oscillation and energy injection control," Journal of Power Electronics, vol. 11, no. 3, pp. 256-263, 2011.

[161] P. Si, A. P. Hu, S. Malpas, and D. Budgett, "A frequency control method for regulating wireless power to implantable devices," IEEE Trans. Biomed. Circuits Syst., vol. 2, pp. 22-29, March 2008.

[162] M. Moghaddami and A. Sarwat, "Self-tuning variable frequency controller for inductive electric vehicle charging with multiple power levels," IEEE Trans. Transport. Electrific., vol. 3, pp. 488-495, June 2017.

[163] S. M. Tayebi and I. Batarseh, "Analysis and optimization of variable-frequency soft-switching peak current mode control techniques for microinverters," IEEE Trans. Power Electron., vol. 33, pp. 1644-1653, Feb 2018.

[164] T. Mishima and E. Morita, "High-frequency bridgeless rectifier based zvs multiresonant converter for inductive power transfer featuring high-voltage ganhfet," IEEE Transactions on Industrial Electronics, vol. 64, pp. 9155-9164, Nov 2017.

[165] H. Li, J. Fang, S. Chen, K. Wang, and Y. Tang, "Pulse density modulation for maximum efficiency point tracking of wireless power transfer systems," IEEE Transactions on Power Electronics, vol. 33, pp. 5492-5501, June 2018.

[166] Y. Sun, H. Zhang, A. P. Hu, C. Tang, and L. Xiang, "The recognition and control of nonideal soft-switching frequency for wireless power transfer system based on waveform identification," IEEE Transactions on Power Electronics, vol. 32, pp. 6617-6627, Aug 2017.

[167] J. Tian and A. P. Hu, "A dc-voltage-controlled variable capacitor for stabilizing the zvs frequency of a resonant converter for wireless power transfer," IEEE Transactions on Power Electronics, vol. 32, pp. 2312-2318, March 2017.

[168] A. Safaee and K. Woronowicz, "Time-domain analysis of voltage-driven series-series compensated inductive power transfer topology," IEEE Transactions on Power Electronics, vol. 32, pp. 4981-5003, July 2017.

[169] C. S. Tang, Y. Sun, Y. G. Su, S. K. Nguang, and A. P. Hu, "Determining multiple steady-state zcs operating points of a switch-mode contactless power transfer system," IEEE Transactions on Power Electronics, vol. 24, pp. 416425, Feb 2009. 
[170] K. Colak, E. Asa, M. Bojarski, D. Czarkowski, and O. C. Onar, "A novel phase-shift control of semibridgeless active rectifier for wireless power transfer," IEEE Transactions on Power Electronics, vol. 30, pp. 6288-6297, Nov 2015.

[171] D. Kim and D. Ahn, "Self-tuning lcc inverter using pwm-controlled switched capacitor for inductive wireless power transfer," IEEE Transactions on Industrial Electronics, pp. 1-1, 2018.

[172] M. Moghaddami, A. Sundararajan, and A. I. Sarwat, "A power-frequency controller with resonance frequency tracking capability for inductive power transfer systems," IEEE Trans. Ind. Appl., vol. 54, pp. 1773-1783, March 2018.

[173] M. Moghaddami, A. Cavada, and A. I. Sarwat, "Soft-switching self-tuning hbridge converter for inductive power transfer systems," in 2017 IEEE Energy Conversion Congress and Exposition (ECCE), pp. 4388-4392, Oct 2017.

[174] M. Moghaddami, A. Sundararajan, and A. I. Sarwat, "A self-tuning variable frequency control for multi-level contactless electric vehicle charger," in 2016 IEEE International Conference on Power Electronics, Drives and Energy Systems (PEDES), pp. 1-5, Dec 2016.

[175] M. Moghaddami, A. Moghadasi, and A. I. Sarwat, "A single-stage three-phase ac-ac converter for inductive power transfer systems," in 2016 IEEE Power and Energy Society General Meeting (PESGM), pp. 1-5, July 2016.

[176] R. L. Steigerwald, "A comparison of half-bridge resonant converter topologies," IEEE Trans. Power Electron., vol. 3, pp. 174-182, Apr 1988.

[177] Q. Ke, W. Luo, G. Yan, and K. Yang, "Analytical model and optimized design of power transmitting coil for inductively coupled endoscope robot," IEEE Trans. Biomed. Eng., vol. 63, pp. 694-706, April 2016.

[178] K. Ishibashi, "Eddy current analysis by boundary element method utilizing impedance boundary condition," IEEE Transactions on Magnetics, vol. 31, pp. 1500-1503, May 1995.

[179] D. Gong, J. Sun, and Z. Miao, "A set-based genetic algorithm for interval many-objective optimization problems," IEEE Trans. Evol. Comput., vol. 22, pp. 47-60, Feb 2018. 
[180] E. K. Burke and G. Kendall, "Search methodologies: Introductory tutorials in optimization and decision support techniques," Springer US.

[181] S. L. Kosakovsky Pond, D. Posada, M. B. Gravenor, C. H. Woelk, and S. D. Frost, "Gard: a genetic algorithm for recombination detection," Bioinformatics, vol. 22, no. 24, pp. 3096-3098, 2006.

[182] G. Monsees, Discrete-time sliding mode control. TU Delft, Delft University of Technology, 2002.

[183] M. Moghaddami and A. I. Sarwat, "Single-phase soft-switched ac-ac matrix converter with power controller for bidirectional inductive power transfer systems," IEEE Transactions on Industry Applications, vol. 54, pp. 3760-3770, July 2018.

[184] M. Moghaddami and A. Sarwat, "Self-tuned single-phase ac-ac converter for bidirectional inductive power transfer systems," in 2017 IEEE Industry Applications Society Annual Meeting, pp. 1-6, Oct 2017.

[185] M. Moghaddami and A. Sarwat, "A three-phase ac-ac matrix converter with simplified bidirectional power control for inductive power transfer systems," in 2018 IEEE Transportation Electrification Conference and Expo (ITEC), pp. 380-384, June 2018.

[186] A. Anzalchi, M. Moghaddami, A. Moghaddasi, A. I. Sarwat, and A. K. Rathore, "A new topology of higher order power filter for single-phase grid-tied voltage-source inverters," IEEE Trans. Ind. Electron., vol. 63, pp. 7511-7522, Dec 2016.

[187] A. Anzalchi, M. Moghaddami, A. Moghadasi, M. M. Pour, and A. I. Sarwat, "Design and analysis of a higher order power filter for grid-connected renewable energy systems," IEEE Trans. Ind. Appl., vol. 53, pp. 4149-4161, Sept 2017.

[188] M. Moghaddami and A. I. Sarwat, "A sensorless conductive foreign object detection for inductive electric vehicle charging systems based on resonance frequency deviation," in 2018 IEEE Industry Applications Society Annual Meeting (IAS), pp. 1-6, Sept 2018.

[189] M. Moghaddami, A. Sundararajan, and A. I. Sarwat, "Sensorless electric vehicle detection in inductive charging stations using self-tuning controllers," in 
2017 IEEE Transportation Electrification Conference (ITEC-India), pp. 1-4, Dec 2017. 
VITA

\section{MASOOD MOGHADDAMI}

July 28, 1986

2004-2009

2009-2011

$2015-2018$
Born, Zanjan, Iran

B.S., Electrical Engineering

Amirkabir University of Technology (Tehran Polytechnic)

Tehran, Iran

M.S., Electrical Engineering

Iran University of Science and Technology

Tehran, Iran

Ph.D., Electrical Engineering

Florida International University, Miami, FL

Miami, Florida

\section{PUBLICATIONS}

1. M. Moghaddami, A. Anzalchi, and A. I. Sarwat, "Single-Stage Three-Phase AC-AC Matrix Converter for Inductive Power Transfer Systems," in IEEE Transactions on Industrial Electronics, vol. 63, no. 10, pp. 6613-6622, Oct. 2016.

2. M. Moghaddami and A. I. Sarwat, "Self-Tuning Variable Frequency Controller for Inductive Electric Vehicle Charging with Multiple Power Levels," in IEEE Transactions on Transportation Electrification, vol. 3, no. 2, pp. 488-495, June 2017.

3. M. Moghaddami and A. I. Sarwat, "A Power-Frequency Controller with Resonance Frequency Tracking Capability for Inductive Power Transfer Systems," in IEEE Transactions on Industry Applications, vol. 54, no. 2, pp. 1773-1783, March-April 2018.

4. M. Moghaddami and A. I. Sarwat, "Single-Phase Soft-Switched AC-AC Matrix Converter with Power Controller for Bidirectional Inductive Power Transfer Systems," accepted in IEEE Transactions on Industry Applications, in press.

5. M. Moghaddami, A. I. Sarwat, and F. de Leon, "Reduction of Stray Loss in Power Transformers Using Horizontal Magnetic Wall Shunts," in IEEE Transactions on Magnetics, vol. 53, no. 2, pp. 1-7, Feb. 2017.

6. M. Moghaddami, A. Moghadasi, and A. I. Sarwat, "An algorithm for fast calculation of short circuit forces in high current busbars of electric arc furnace transformers based on method of images," Electric Power Systems Research, vol. 136, pp. 173-180, Jul. 2016. 
7. M. Moghaddami and A. I. Sarwat, "Generalized Physics-Based Multi-Objective Design Optimization of Magnetic Structures for Inductive Charging Systems," in IEEE Transactions on Industrial Electronics, (in review).

8. A. Anzalchi, M. Moghaddami, A. Moghaddasi, A. I. Sarwat, and A. K. Rathore, "A New Topology of Higher Order Power Filter for Single-Phase Grid-Tied Voltage-Source Inverters," in IEEE Transactions on Industrial Electronics, vol. 63, no. 12, pp. 7511-7522, Dec. 2016.

9. A. Anzalchi, M. Moghaddami, A. Moghadasi, M. Malek Pour, and A. Sarwat, "Design and Analysis of a Higher Order Power Filter for Grid-Connected Renewable Energy Systems," in IEEE Transactions on Industry Applications, vol. 53, no. 5, pp. 4149-4161, Sept.-Oct. 2017.

10. M. Moghaddami, A. Cavada and A. I. Sarwat, "Soft-switching self-tuning Hbridge converter for inductive power transfer systems," 2017 IEEE Energy Conversion Congress and Exposition (ECCE), Cincinnati, OH, USA, 2017, pp. $4388-4392$.

11. M. Moghaddami and A. Sarwat, "Self-tuned single-phase AC-AC converter for bidirectional inductive power transfer systems," 2017 IEEE Industry Applications Society Annual Meeting, Cincinnati, OH, USA, 2017, pp. 1-6.

12. M. Moghaddami, A. Anzalchi, A. Moghadasi and A. Sarwat, "Pareto optimization of circular power pads for contactless electric vehicle battery charger," 2016 IEEE Industry Applications Society Annual Meeting, Portland, OR, 2016, pp. 1-6.

13. M. Moghaddami, A. Anzalchi and A. I. Sarwat, "Finite element based design optimization of magnetic structures for roadway inductive power transfer systems," 2016 IEEE Transportation Electrification Conference and Expo (ITEC), Dearborn, MI, 2016, pp. 1-6.

14. M. Moghaddami, A. Sundararajan and A. I. Sarwat, "Sensorless electric vehicle detection in inductive charging stations using self-tuning controllers," 2017 IEEE Transportation Electrification Conference (ITEC-India), Pune, India, 2017, pp. 1-4.

15. M. Moghaddami and A. I. Sarwat, "Time-Dependent Multi-Physics Analysis of Inductive Power Transfer Systems," 2018 IEEE Transportation Electrification Conference and Expo (ITEC), Long Beach, CA, USA, 2018, pp. 130-134.

16. M. Moghaddami and A. I. Sarwat, "A Three-Phase AC-AC Matrix Converter with Simplified Bidirectional Power Control for Inductive Power Transfer Systems," 2018 IEEE Transportation Electrification Conference and Expo (ITEC), Long Beach, CA, USA, 2018, pp. 380-384.. 